\title{
THE REACTION OF GLASS IN A GAMMA IRRADIATED \\ SATURATED TUFF ENVIRONMENT, PART II \\ DATA PACKAGE FOR ATM-1C AND ATM-8 GLASSES
}

\author{
Work Performed by \\ John K. Bates \\ Thiomas J. Gerding \\ Donald F. Fischer \\ William L. Ebert
}

of

\author{
ARGONNE NATIONAL LABORATORY \\ Chemical Technclogy Division \\ 9700 South Cass Avenue \\ Argonne, Illinois 60439
}

\section{DISCLAIMER}

This report was prepared as an account of work sponsored by an agency of the United Slates Gowernment. Neither the United States Govern.nent not any agency thereof, nut any of their employecs, makes any warranty, express or implied, or assumes any legal liability or responsibility for the aceufac! . completeness, or usefuiness of any informution, apparutus, product, or process disclosed, or represents that its use would not infringe privalely owned rights. Refer. ence herein to any specific commercial product, process, or service by trade natne, tradernark, manufacturer, of otherwise does nol necessurily constitule or imply its endorsement, recommendation. or favoring by the Uniled Siates Gavernment or any agency thersof. The views and s,pinions of authuss expressed hetein do nol necessarily state uf seflect those of the United States Government os any agency thereof. 
DSTHAIMER

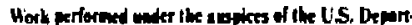

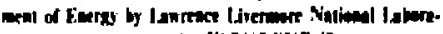

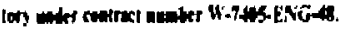

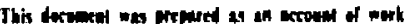

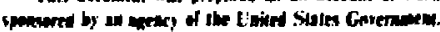

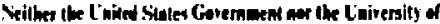

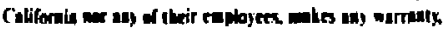

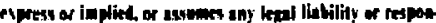

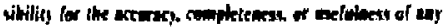

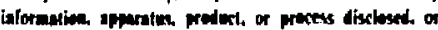

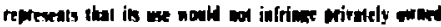
rizhls. Reference hereiw to any specific commercial prot

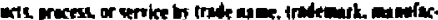

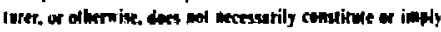

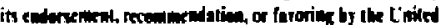

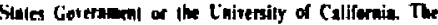

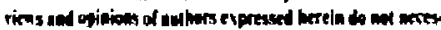

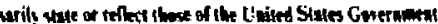

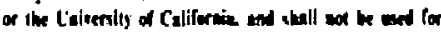

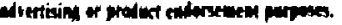

Prepared by Nernda Nuclear Wate Storage lnwertigations (NNWSn) Project particlpants as part of the Civtlin Radlactive Warto Mangement Program. The NNWSI Profect I manged by the Warte Marugement Project office of the U.S. Depurtment of Encrcy, Nevad Opertilons offlet. NWwSI Project work to fponered by the Orflee of Geologile Repositocies of the DOE Office of Civiling Redionctive Wuste Managetant. 
TABLE OF COHTENTS

Introduction .................... 1

Table A-1. Composition of EJ-13 Leachants Used in Gama Experiments ............... 3

Table A-2. Analysis of ATM-1C and ATM-8 Glasses ......... 5

Table A-3a, Experimental Matrix and Component Weight Changes, ATM-1c Glass, 2R Experiments ....... T T

Table A-3b, Experimental Matrix and Component Weight Changes, ATH-8 Glasses ........... 10

Table A-3C. Test Matrix and Component Weight Changes, Blanks, 2R Experiments ............ 13

Table A-4. FY 1986 Gand Irradiation Experinents Matrix ..... 17

Table A-5a. Experimental Matrix and Selected Results for the OR Experfinents Done with Teflon Gaskets ...... 24

Table A-5b. Experimental Matrix and Selected Results for Prelininary Experiments Done Without

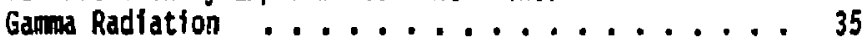

Table A-6a. Cation Analyses, ATM-1C Glass ........... 37

Table A-6b. Cation Analyses, ATM-8 Glass . . . . . . . . . 42

Table A-6c, Cation Analyses, Blanks ............... 47

Table A-7. Cation Analyses, $1 R$ and OR (Blanks, Silicon Gaskets) Experinents .......... 51

Table A-8a. Cation Analyses, OR (Teflcin Gasket) Experiments .... 66

Table A-8b. Solution Results for Preliminary ATM-1C Experfments Done Without Radiation ........ 81

Table A-9. pH and Anion Analyses - Blank Samples.........83

Table A-10. Anion Results for the IR and OR (stlicone Gaskets) Experiments ............ 87

Table A-11. Anton Results for the OR (Teflon) Experiments..... 93 


\section{TABLE OF CONTENTS - CONE'd}

Page

Table A-12. Np and Pu Release from ATM-8 Glass . . . . . . . . 97

Table A-13. Np and Pu Results from the $1 R$ Experiments . . . . . 100

Table A-14. Np and Pu Results from the OR (Teflon

Gasket) Experiments ............ 105

Table A-15. SEM/EOS Analyses Performed on ATM-1C,

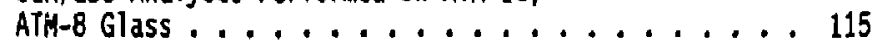

Table A-16. ATM-1C Glasses Profiled Using SIMS . . . . . . . 117

fig. A-1a-f. SIMS Profiles for ATM-IC Glass . . . . . . . . . 118

Fig. A-2. SIMS Spectra of ATM-1C Glass ........... 121

Fig. A-3. SIMS Spectra of ATM-1C Glass ............ 123

References ........................ 125 
THE REACTION OF GLASS IM A GANA IRRADIATED

SATURATED TUFF ENVIRONMEMT, PART II

DATA PACKAGE FOR ATM-IC AHD ATTH-8 GLASSES

by

John K. Bates, Thomas J, Gerding, Donald f. Flscher, and Willian L. Ebert

\section{INTRODUCTIOK}

A series of experiments have been perforned in support of the Nevada Nuclear Waste Storage Investigations (MnisI) profect that investigate the effects of gasoa radiation on groundwater and glass reaction. Experiments have been done in a gasen radiation field at exposure rates ranging between $2 \times 13^{5}$ and $0 \mathrm{R} / \mathrm{h}$, and have been performed over a period of four years. The results of these experiments using ATH-1C and ATH-8 glasses have been described in an article pubilished in the Journal of Materlals Research [BATES-1]. A thorough discussion of the experimenta] procedures has been presented previously [BATES-2].

In this report, all the data that have been generated during these experiaents and which were used in writing the article are presented. The report consists of a series of Tables that provide the

1). groundwater compositions

2) glass compositions

3) experimental matrices and selected results

4) cation analyses

5) anion analyses

6) Hp and Pu analyses

7) SEK/EDS analyses

8) Sins analyses

Each Table is preceded by a cover sheet that describes the contents of the Table and expiains the colum annotations. Experiaents were conducted at exposure rates of $2 \times 10^{5}(2 R), 1 \times 10^{3}$ (1R), and 0 (OR) $R / h$. 
TABLE A-1

Composition of EJ-13 Leachants Used in Gamma Experiments

Water was equilibrated prior to the inftiation of a matrix and was used throughout the matrix uniess the composition changed significantly. The water was made according to procedures given in [BATES-2]. 
Table A-1. Composition of EJ-13 Leachants Used in Gama Experiments

\begin{tabular}{|c|c|c|c|c|}
\hline \multirow[b]{2}{*}{ Species } & \multicolumn{4}{|c|}{ Composition (ppm) } \\
\hline & $1 R^{a}$ & $3 R^{b}, O R b$ & $2 R C$ & $O R^{d}$ \\
\hline $\mathrm{Ma}$ & 44.4 & 49.3 & 46.5 & 51.1 \\
\hline Si & 34.5 & 35.7 & 34.4 & 35.9 \\
\hline $\mathrm{Ca}$ & 8.10 & 7.10 & 9.08 & 6.78 \\
\hline$k$ (estimated) & 6.0 & 6.0 & 6.0 & 6.0 \\
\hline $\mathrm{Mg}$ & 0.34 & 0.25 & 0.96 & 0.15 \\
\hline Al & 0.30 & 0.43 & 0.63 & 0.71 \\
\hline$B$ & 0.14 & 0.17 & 0.16 & 0.15 \\
\hline$S r$ & 0.044 & 0.036 & 0.045 & 0.037 \\
\hline Li & 0.044 & 0.041 & 0.044 & NA \\
\hline $\mathrm{Fe}$ & $<0.01$ & $<0.01$ & 0.01 & $<0.01$ \\
\hline$U$ & $<0.001$ & $<0.001$ & 0.0024 & $<0.001$ \\
\hline $\mathrm{HCO}_{3}-d$ & 117 & 140 & 122 (estimated) & 94 \\
\hline $\mathrm{SO}_{4}^{-}$ & 18.0 & 18.0 & 17.3 & 20.3 \\
\hline $\mathrm{NO}_{3}-$ & 6.1 & 7.6 & 7.6 & 7.9 \\
\hline $\mathrm{Cl}^{-}$ & 7.0 & 8.2 & 7.15 & 7.8 \\
\hline$F^{-}$ & 2.5 & 2.6 & 2.4 & 2.4 \\
\hline $\mathrm{NO}_{2}^{-}$ & $<0.3$ & $<0.3$ & $<0.3$ & $<0.3$ \\
\hline $\mathrm{pH}$ & 7.6 & 8.1 & 8.1 & 8.5 \\
\hline
\end{tabular}

aLeachant used for 56-, 91-, 181-, and 278-day $I R$ and OR experiments. bleachant used for 14- and 28-day IR experiments and all OR silicone gasket experiments.

CLeachant used in 2R experiments.

dLeachant used in all OR Teflon gasket experiments. 
TABLE A-2

Analyses of ATH-IC and ATM-8 Glasses

The ATM-1C and ATM-8 glasses used in these experiments were supplied by the Materials Characterization Center (MCC). For tive $2 R$ experiments, the disks were supplied as-cut by the MCC. In the $1 R$ and $0 R$ experiments glass bars supplied by the HCC were cored and sectloned at ANL [BATES-2]. The glasses were analyzed by the MCC, LLNL, and ANL. These oxide analyses are given in the Table together with an element fraction $\left(f_{f}\right)$ in the glass that was subsequently used in all normalized mass loss calculations.

At ANL the analyses were done by acld dissolution of the glass followed by ICP analysis of everything except Si, B, Cs, and the transuranic elements. Si was analyzed by colarimetry (after alkali carbonate fusion), $B$ by isotopic dilution mass spectrometry (after alkall carbonate fusion), Cs by atomic absorption, and transuranic elements by alpha or gama spectroscopy.

The LLNL values were obtained from F. Bazan, and the MCC values were taken fron reports PNL-5577-1 (ATM-1C) and PNL-5577-8 (ATM-8). If a value is not reported, then no analysis was done. The $f_{f}$ values are the element weight percent in the glass. 
Tabłe A-2. Analys is of ATH-1C and ATM-8 Glasses (oxide wt $x$ )

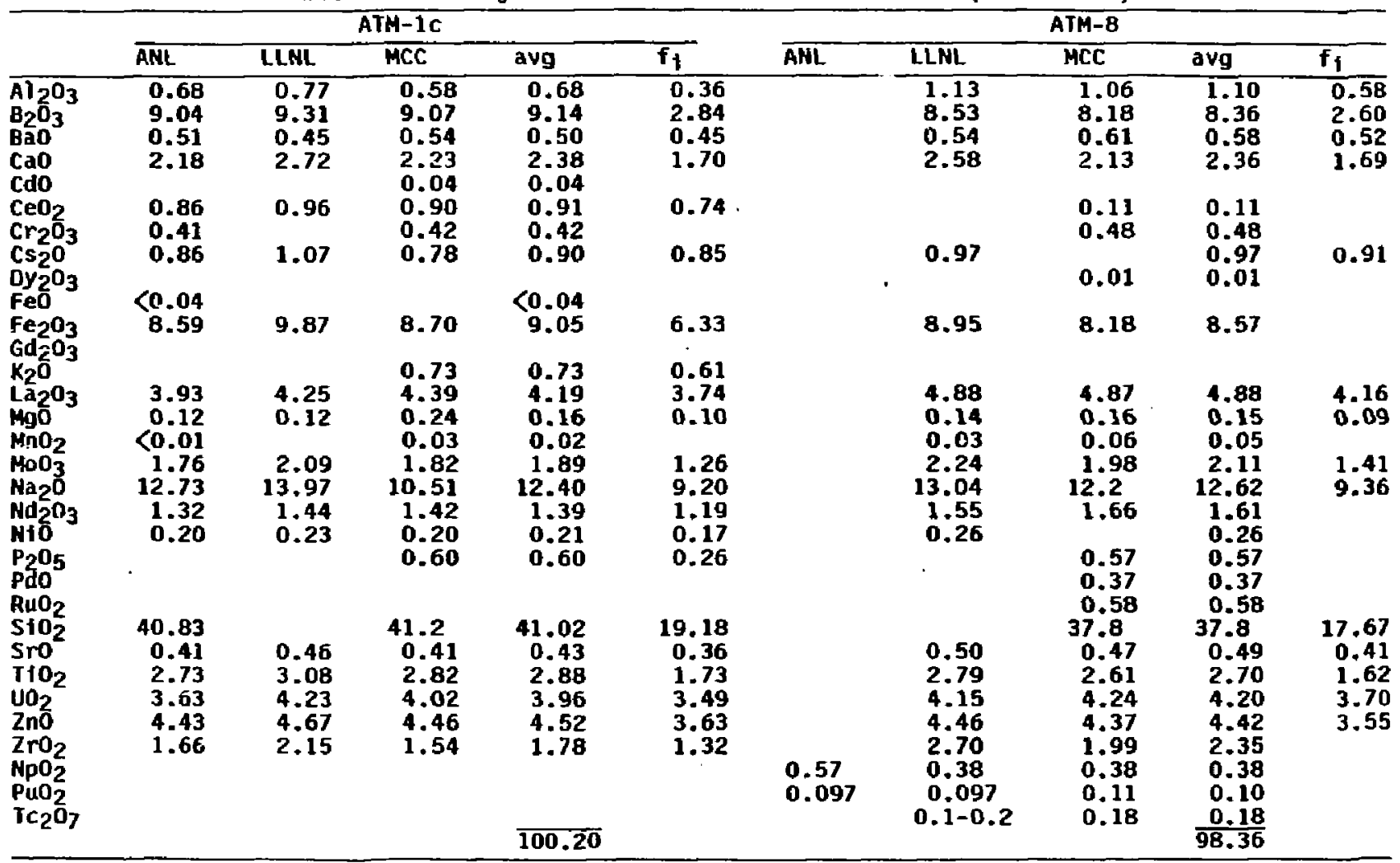




$$
\text { TABLE A-3a,b,c }
$$

Experimental Matrfx and Component Weight Changes, ATM-1C (3a), ATM-8 (3b), and Blank (3c), 2R Experiments

The experimental matrix and selected measurements used in the $2 R$ experiments are presented in this Table. The LPE liquid weight is the weight of the liquid submitted for cation analysis. 
Table A-3a. Experimental Matrix and Component Weight Changes, ATM-lc Glass, $2 R$ Experiments

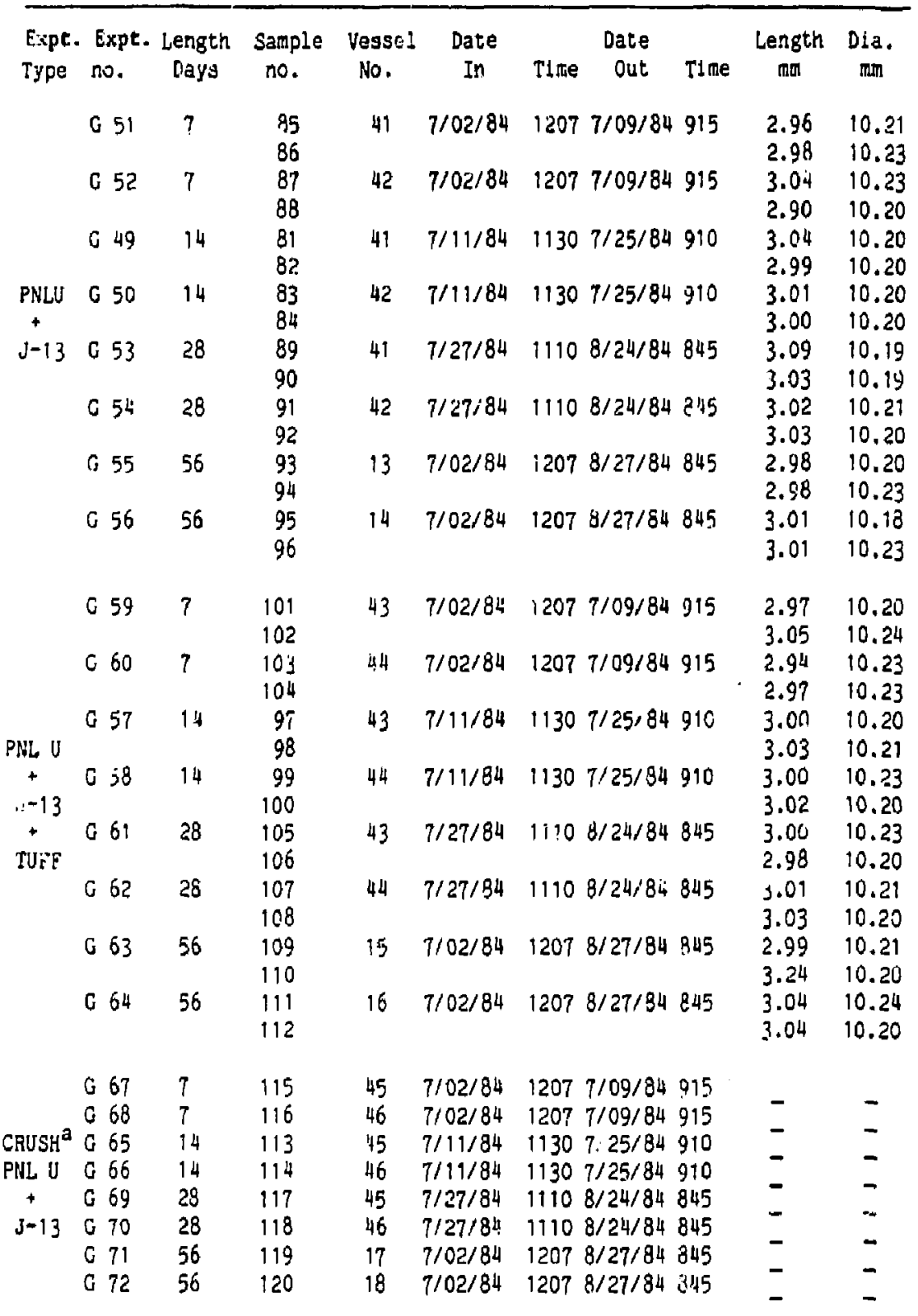

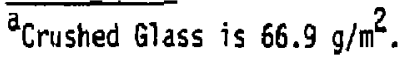


Table A-3a (Cont'd)

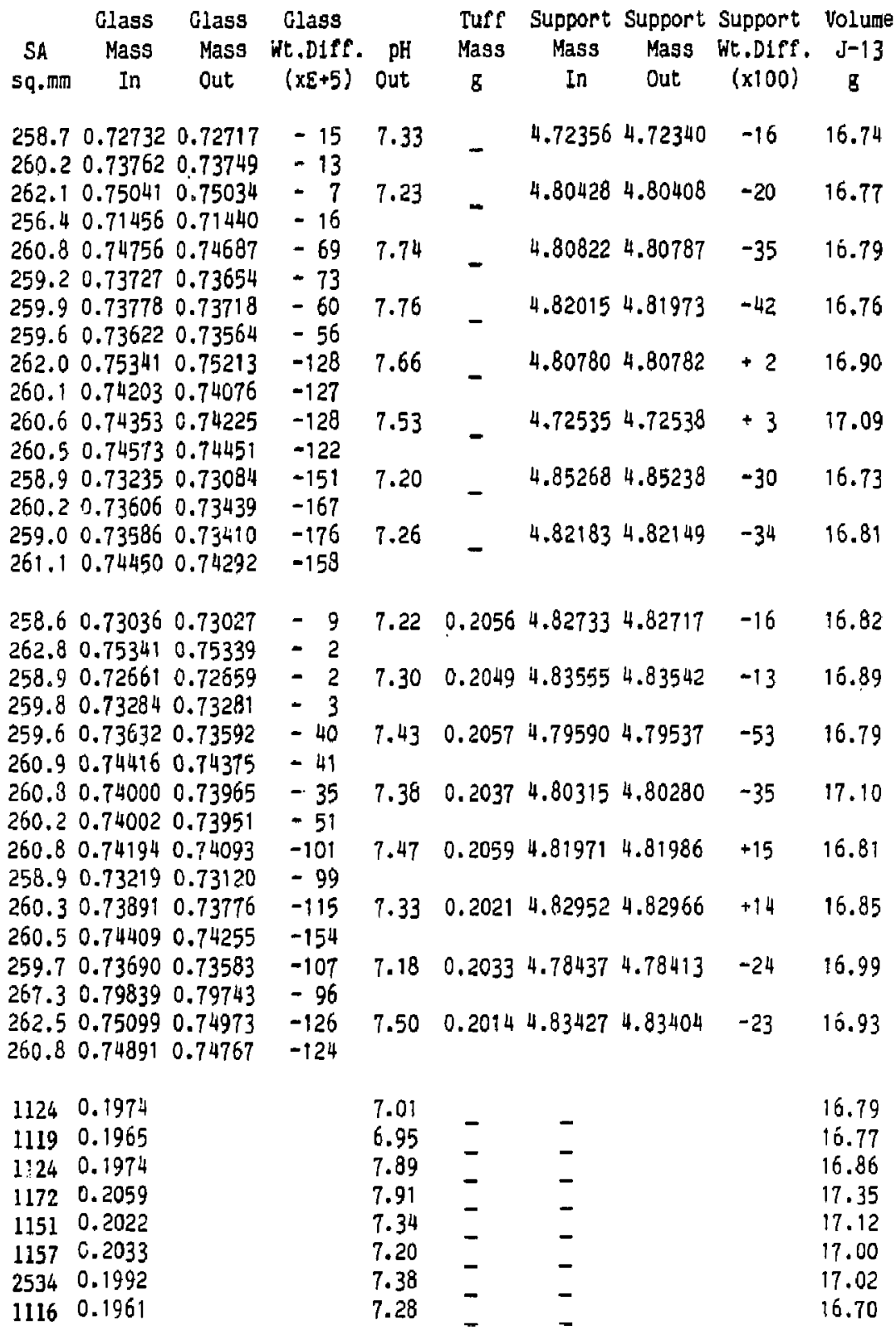


Table A-3a (Cont'd)

\begin{tabular}{|c|c|c|c|}
\hline $\begin{array}{c}\text { Complete } \\
\text { Assembly } \\
\text { I: }\end{array}$ & $\begin{array}{l}\text { Complete } \\
\text { Assembly } \\
\text { Out }\end{array}$ & $\begin{array}{c}\text { Assembly } \\
\text { Wt. .Diff. } \\
(x 100)\end{array}$ & $\begin{array}{l}\text { LPE } \\
\text { LI quid } \\
\text { Wt }\end{array}$ \\
\hline 135.67 & 135.67 & 0 & 12.41 \\
\hline 135.92 & 135.91 & -1 & 12,36 \\
\hline 135.87 & 135.86 & -1 & 12.47 \\
\hline 135.71 & 135.70 & -1 & $: 2.50$ \\
\hline 135.96 & 135.90 & -6 & 13.02 \\
\hline 136.12 & 136.09 & -3 & 14.65 \\
\hline 135.96 & 135.93 & -3 & 14.07 \\
\hline 135.99 & 135.94 & -5 & 14.25 \\
\hline 136.87 & 136.86 & -1 & 11.94 \\
\hline 137.27 & 137.27 & 0 & 11.10 \\
\hline 136.92 & 136.90 & -2 & 11.46 \\
\hline 137.58 & 137.56 & -2 & 11.88 \\
\hline 136.95 & 136.92 & -3 & 13.75 \\
\hline 137.43 & 137.39 & -4 & 13.46 \\
\hline 135.6 & 135.65 & -4 & 13.71 \\
\hline 137.71 & 137.68 & -3 & 13.46 \\
\hline $\begin{array}{l}131.64 \\
130.95 \\
131.75 \\
131.39 \\
132.01 \\
131.06 \\
130.37 \\
129.99\end{array}$ & $\begin{array}{l}131.64 \\
130.94 \\
131.69 \\
130.84 \\
131.97 \\
130.97 \\
130.34 \\
129.95\end{array}$ & $\begin{array}{r}0 \\
-1 \\
-6 \\
-55 \\
-4 \\
-9 \\
-3 \\
-4\end{array}$ & $\begin{array}{l}11.44 \\
11.07 \\
12.08 \\
11.61 \\
14.20 \\
14.12 \\
14.08 \\
13.79\end{array}$ \\
\hline
\end{tabular}


Table A-3b. Experimental Matrix and Component Weight Changes, ATM-8 Glasses

\begin{tabular}{|c|c|c|c|c|c|c|c|c|c|c|}
\hline \multirow[t]{4}{*}{$\begin{array}{l}\text { Expt. } \\
\text { Type }\end{array}$} & $\begin{array}{l}\text { Expt. } \\
\text { no. }\end{array}$ & $\begin{array}{c}\text { Length } \\
\text { Days }\end{array}$ & $\begin{array}{c}\text { Sample } \\
\text { no. }\end{array}$ & $\begin{array}{l}\text { Vessel } \\
\text { ik. }\end{array}$ & $\begin{array}{l}\text { Date } \\
\text { In }\end{array}$ & Tine & $\begin{array}{c}\text { Date } \\
\text { Out }\end{array}$ & Time & $\begin{array}{c}\text { Length } \\
m m\end{array}$ & $\begin{array}{c}\text { Dia. } \\
\text { man }\end{array}$ \\
\hline & \& 75 & 7 & $\begin{array}{l}125 \\
126\end{array}$ & 47 & $7 / 02 / 84$ & 1207 & $7 / 09 / 84$ & 915 & $\begin{array}{l}2.87 \\
3.00\end{array}$ & $\begin{array}{l}10.16 \\
10.18\end{array}$ \\
\hline & G 76 & 7 & $\begin{array}{l}127 \\
128\end{array}$ & 48 & $7 / 02 / 84$ & 1207 & $7 / 09 / 84$ & 915 & $\begin{array}{l}2.94 \\
2.99\end{array}$ & $\begin{array}{l}10.17 \\
10.17\end{array}$ \\
\hline & G 73 & 14 & $\begin{array}{l}121 \\
122\end{array}$ & 47 & $7 / 11 / 84$ & 1130 & $7 / 25 / 84$ & 910 & $\begin{array}{l}3.12 \\
3.10\end{array}$ & $\begin{array}{l}10.18 \\
10.16\end{array}$ \\
\hline PHL A & 674 & 14 & $\begin{array}{l}123 \\
124\end{array}$ & 48 & $7 / 11184$ & 1130 & $7 / 25 / 84$ & 910 & $\begin{array}{l}2.87 \\
2.84\end{array}$ & $\begin{array}{l}10.18 \\
10.17\end{array}$ \\
\hline$J-13$ & $\begin{array}{l}\text { G } 77 \\
\text { C } 78\end{array}$ & $\begin{array}{l}28 \\
28\end{array}$ & $\begin{array}{l}129 \\
130 \\
131 \\
132\end{array}$ & 47 & $\begin{array}{l}7 / 27 / 84 \\
7 / 27 / 84\end{array}$ & $\begin{array}{l}1110 \\
1110\end{array}$ & $8 / 24 / 84$ & 845 & $\begin{array}{l}2.92 \\
2.95 \\
2.80 \\
2.90\end{array}$ & $\begin{array}{l}10.16 \\
10.18 \\
10.18 \\
10.18\end{array}$ \\
\hline & G 79 & 56 & $\begin{array}{l}133 \\
134\end{array}$ & 19 & $7 / 02 / 84$ & 1207 & $8 / 27 / 84$ & 845 & $\begin{array}{l}2.97 \\
2.90\end{array}$ & $\begin{array}{l}10.16 \\
10.15\end{array}$ \\
\hline & 080 & 56 & $\begin{array}{l}135 \\
136\end{array}$ & 20 & $7 / 02 / 84$ & 1207 & $8 / 27 / 84$ & 845 & $\begin{array}{l}3.08 \\
3.00\end{array}$ & $\begin{array}{l}10.18 \\
10.16\end{array}$ \\
\hline & C 83 & 7 & $\begin{array}{l}141 \\
142\end{array}$ & 49 & $7 / 02 / 84$ & 1207 & $7 / 09 / 84$ & 915 & $\begin{array}{l}2.9: \\
2.99\end{array}$ & $\begin{array}{l}10.17 \\
10.19\end{array}$ \\
\hline & C 84 & 7 & $\begin{array}{l}143 \\
144\end{array}$ & 50 & $7 / 02 / 84$ & 1207 & $7 / 09 / 84$ & 915 & $\begin{array}{l}2.89 \\
3.01\end{array}$ & $\begin{array}{l}10.18 \\
i 0.18\end{array}$ \\
\hline PNL A & C 81 & 14 & $\begin{array}{l}137 \\
138\end{array}$ & 49 & $7 / 11 / 84$ & 1130 & $7 / 25 / 84$ & 910 & $\begin{array}{l}2.98 \\
2.92\end{array}$ & $\begin{array}{l}10.16 \\
10.18\end{array}$ \\
\hline$j-13$ & 082 & 14 & $\begin{array}{l}139 \\
140\end{array}$ & 50 & $7: 11 / 84$ & 1130 & $7 / 25 / 84$ & 910 & $\begin{array}{l}2.93 \\
2.90\end{array}$ & $\begin{array}{l}10.18 \\
10.17\end{array}$ \\
\hline$\stackrel{+}{\text { TUEF }}$ & 6 85 & 28 & $\begin{array}{l}145 \\
146\end{array}$ & 49 & $7 / 27 / 84$ & 1110 & $8 / 24 / 84$ & 845 & $\begin{array}{l}2.9 r \\
2.86\end{array}$ & $\begin{array}{l}10.16 \\
10.17\end{array}$ \\
\hline & G 86 & 28 & $\begin{array}{l}147 \\
148\end{array}$ & 50 & $7 / 27 / 84$ & 1110 & $8 / 24 / 84$ & 845 & $\begin{array}{l}2.91 \\
2.88\end{array}$ & $\begin{array}{l}10.18 \\
10.20\end{array}$ \\
\hline & G 87 & 56 & $\begin{array}{l}149 \\
150\end{array}$ & 21 & $7 / 02 / 24$ & 1207 & $8 / 27 / 84$ & 845 & $\begin{array}{l}2.90 \\
2.88\end{array}$ & $\begin{array}{l}10.19 \\
10.18\end{array}$ \\
\hline & G 88 & 50 & $\begin{array}{l}151 \\
152\end{array}$ & 22 & $7 / 02 / 84$ & 1207 & $8 / 27 / 84$ & 845 & $\begin{array}{l}3.00 \\
3.04\end{array}$ & $\begin{array}{l}10.19 \\
10.15\end{array}$ \\
\hline CRUSH $^{2}$ & $\begin{array}{l}\text { C } 91 \\
\text { c } 92 \\
\text { C } 89\end{array}$ & $\begin{array}{l}7 \\
7 \\
14\end{array}$ & $\begin{array}{l}155 \\
156 \\
153\end{array}$ & $\begin{array}{l}55 \\
52 \\
51\end{array}$ & $\begin{array}{l}7 / 02 / 84 \\
7 / 02 / 84 \\
7 / 11 / 84\end{array}$ & $\begin{array}{l}1207 \\
1207 \\
1130\end{array}$ & $\begin{array}{l}7 / 09 / 84 \\
7 / 09 / 84 \\
7 / 25 / 84\end{array}$ & $\begin{array}{l}915 \\
915 \\
910\end{array}$ & - & 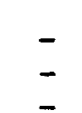 \\
\hline PNL $A$ & C 90 & 14 & 154 & 52 & $7 / 11 / 84$ & 1130 & $7 / 25 / 84$ & 910 & - & - \\
\hline$\stackrel{+}{5-13}$ & C 93 & 28 & $\begin{array}{l}157 \\
158\end{array}$ & 55 & $7 / 27 / 84$ & 1110 & $8 / 24 / 84$ & 845 & - & 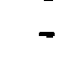 \\
\hline $\mathrm{S}-13$ & $\begin{array}{l}\text { C } 94 \\
\text { C } 95 \\
\text { C } 96\end{array}$ & $\begin{array}{l}26 \\
56 \\
56\end{array}$ & $\begin{array}{l}158 \\
159 \\
160\end{array}$ & $\begin{array}{l}22 \\
23 \\
24\end{array}$ & $\begin{array}{l}7 / 02 / 84 \\
7 / 02 / 84\end{array}$ & $\begin{array}{l}1110 \\
1207 \\
1207\end{array}$ & $\begin{array}{l}8 / 24 / 84 \\
8 / 27 / 84 \\
8 / 27 / 84\end{array}$ & $\begin{array}{l}845 \\
845 \\
845\end{array}$ & - & - \\
\hline
\end{tabular}

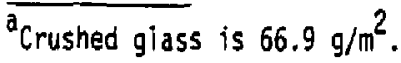


Table A-3b (Cont'd)

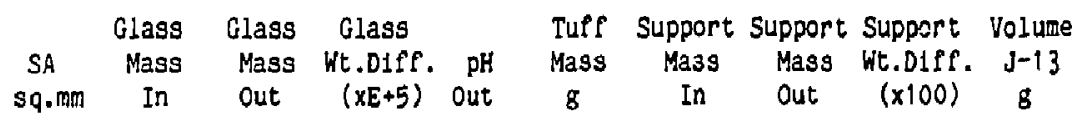

\begin{tabular}{|c|c|c|c|c|c|c|c|c|}
\hline $\begin{array}{r}253.8 \\
.258 .7\end{array}$ & $\begin{array}{l}0.71138 \\
0.74209\end{array}$ & $\begin{array}{l}0.71132 \\
0.74203\end{array}$ & $\begin{array}{l}-6 \\
-6\end{array}$ & 6.86 & & 4.768924 .76874 & -18 & 16.53 \\
\hline 256.4 & 0.72981 & 0.72979 & -2 & 7.00 & & 4.845374 .84521 & -16 & 16.70 \\
\hline 258.0 & 0.74543 & 0.74537 & -6 & & & & & \\
\hline $\begin{array}{l}262.6 \\
261.1\end{array}$ & $\begin{array}{l}0.73298 \\
0.72766\end{array}$ & $\begin{array}{l}0.73232 \\
0.72705\end{array}$ & $\begin{array}{l}-66 \\
-61\end{array}$ & 6.80 & & $4.84417 \quad 4.84389$ & -28 & 16.96 \\
\hline 254.6 & 0.71349 & 0.71280 & -69 & 7.18 & & 4.838694 .83828 & -41 & 16.42 \\
\hline 253.2 & 0. & 0.69157 & .43 & & & & & \\
\hline 255.3 & 0.72739 & 0.72624 & -115 & 6.92 & & 4.853574 .85351 & -6 & 16.52 \\
\hline 257.1 & 0.7 & 0.72059 & -116 & & & & & \\
\hline $\begin{array}{l}252.3 \\
255.5\end{array}$ & $\begin{array}{l}0.7 \\
0.7\end{array}$ & $\begin{array}{l}0.69876 \\
0.72228\end{array}$ & $\begin{array}{l}-127 \\
-118\end{array}$ & 7.01 & & $4.87142 \quad 4.87 \div 43$ & +1 & 16.36 \\
\hline 56.9 & 0.73377 & 0.73280 & -97 & 7.50 & & 4.803084 .80278 & -30 & 16.49 \\
\hline $\begin{array}{l}54.3 \\
61.3\end{array}$ & & $\begin{array}{l}0.114 द 9 \\
0.75602\end{array}$ & -114 & 7.37 & & 4 & -33 & 7 \\
\hline 57.9 & 0.7 & 0.74479 & -113 & & & 7. & תנ & \\
\hline 37.7 & 0.74229 & 0.74225 & -4 & 7.05 & 0.2030 & 4.843124 .84313 & +1 & 16.70 \\
\hline 58.8 & 0.74360 & 0.74367 & +7 & & & & & \\
\hline 255.2 & 2.72328 & 0.72323 & -5 & 7.02 & 0.2029 & 4.862384 .86239 & +1 & 16.59 \\
\hline 59.0 & 0.7 & 0.70744 & -13 & & & & & \\
\hline $\begin{array}{l}257.3 \\
256.2\end{array}$ & $\begin{array}{l}0.73352 \\
0.72434\end{array}$ & $\begin{array}{l}0.73320 \\
0.72394\end{array}$ & $\begin{array}{l}-32 \\
-40\end{array}$ & 7.20 & 0.2020 & $4.85391 \quad 4.85353$ & -38 & 16.73 \\
\hline $\begin{array}{l}256.5 \\
255.1\end{array}$ & 0.73116 & $\begin{array}{l}0.73068 \\
0.72558\end{array}$ & $\begin{array}{l}-48 \\
-42\end{array}$ & 7.23 & 0.2040 & 4.712704 .71238 & -32 & 16.51 \\
\hline 254.7 & 0.70415 & 0.70393 & -22 & 7.20 & 0.2028 & $4.88467 \quad 4.88463$ & -4 & 16.43 \\
\hline 53.8 & 0.69068 & 0.69035 & -33 & & & & & \\
\hline 255.9 & 0.71261 & 0.71169 & -92 & 7.09 & 0.2071 & $4.71234 \quad 4.71231$ & -3 & 16.53 \\
\hline 255.7 & 0.6 & 0.69740 & -131 & & & & & \\
\hline $\begin{array}{l}255.9 \\
254.9\end{array}$ & 0.72063 & 0.72009 & $\begin{array}{l}-54 \\
-53\end{array}$ & 7.48 & 0.2030 & 4.820514 .82018 & -33 & 16.54 \\
\hline 259.1 & 0.73457 & 0.73437 & -20 & $7.6 !$ & 0.2028 & $4.82446 \quad 4.82418$ & -28 & 16.71 \\
\hline 258.8 & 0.74094 & 0.74088 & -6 & & & & & \\
\hline 1078 & 0.1894 & & & 6.80 & & & & 16.1 \\
\hline 1147 & 0.2015 & & & 6.95 & & & & 17.22 \\
\hline 1033 & 0.1815 & & & 7.16 & & & & 15.53 \\
\hline 1042 & 0.1831 & & & 7.04 & & & & 15.60 \\
\hline 1064 & 0.1868 & & & 7.37 & & & & 15.82 \\
\hline 1096 & 0.1925 & & & 7.42 & & & & $i 6.18$ \\
\hline 1089 & 0.1912 & & & 7.74 & & & & 16.29 \\
\hline 1129 & 0.1983 & & & 7.68 & & - & & 16.88 \\
\hline
\end{tabular}


Table A-3b (Cont'd)

Complete Complete Assembly LPE
Assembly Assenbly Wt. Dlpf. Liquid
In
Out $(x 100)$ wt

$\begin{array}{llll}136.59 & 136.58 & -1 & 11.76 \\ 136.91 & 136.90 & -1 & 12.06 \\ 137.13 & 137.10 & -3 & 13.20 \\ 136.52 & 136.51 & -1 & 12.89 \\ 136.61 & 136.58 & -3 & 13.37 \\ 136.52 & 136.47 & -5 & 13.63 \\ 134.72 & 134.56 & -12 & 13.17 \\ 133.90 & 133.84 & -6 & 13.93 \\ 138.58 & 138.57 & -1 & 9.77 \\ 138.20 & 138.20 & 0 & 10.30 \\ 138.48 & 138.47 & -1 & 12.21 \\ 137.89 & 137.84 & -5 & 12.12 \\ 138.15 & 138.13 & -2 & 13.03 \\ 137.91 & 137.88 & -3 & 11.93 \\ 135.00 & 134.89 & -11 & 13.02 \\ 136.26 & 136.14 & -12 & 13.16\end{array}$

$\begin{array}{llll}130.57 & 130.66 & -1 & 10.90\end{array}$

$\begin{array}{llll}131.33 & 131.32 & -1 & 12.09\end{array}$

$\begin{array}{llll}130.35 & 130.33 & -2 & 11.64\end{array}$

$\begin{array}{llll}129.65 & 129.62 & -3 & 11.64\end{array}$

$\begin{array}{llll}130.39 & 130.35 & -4 & 12.55\end{array}$

$\begin{array}{llll}130.26 & 130.22 & -4 & 12.89\end{array}$

$\begin{array}{llll}129.38 & 129.32 & -6 & 12.94\end{array}$

$\begin{array}{llll}130.09 & 130.04 & -5 & 13.74\end{array}$ 
Tabie A-3c. Test Matrix and Component Height Changes, Blanks, 2R Experiments

\begin{tabular}{|c|c|c|c|c|c|c|c|c|c|c|}
\hline $\begin{array}{l}\text { Test } \\
\text { Type }\end{array}$ & $\begin{array}{l}\text { Test } \\
\text { no. }\end{array}$ & $\begin{array}{l}\text { Length } \\
\text { Days }\end{array}$ & $\begin{array}{l}\text { Sample } \\
\text { no. }\end{array}$ & $\begin{array}{l}\text { Vessel } \\
\text { No. }\end{array}$ & $\begin{array}{c}\text { Date } \\
\text { In }\end{array}$ & T1me & $\begin{array}{r}\text { Date } \\
\text { Out }\end{array}$ & Time & $\begin{array}{l}\text { Length } \\
\text { mm }\end{array}$ & $\underset{\mathrm{mm}}{\mathrm{DHa}}$ \\
\hline $\begin{array}{l}\text { BLANKS } \\
\text { VESSEL } \\
+ \\
j-1\}\end{array}$ & $\begin{array}{ll}G & 99 \\
G & 100 \\
G & 97 \\
G & 98 \\
G & 101 \\
G & 102 \\
G & 103 \\
G & 104\end{array}$ & $\begin{array}{l}7 \\
7 \\
14 \\
14 \\
28 \\
28 \\
56 \\
56\end{array}$ & $\begin{array}{l}\text { - } \\
- \\
- \\
- \\
-\end{array}$ & $\begin{array}{l}35 \\
54 \\
53 \\
54 \\
35 \\
54 \\
25 \\
26\end{array}$ & $\begin{array}{l}7 / 02 / 84 \\
7 / 02 / 84 \\
7 / 11 / 84 \\
7 / 11 / 84 \\
7 / 27 / 84 \\
7 / 27 / 84 \\
7 / 02 / 84 \\
7 / 02 / 84\end{array}$ & $\begin{array}{l}1207 \\
1207 \\
1130 \\
1130 \\
1110 \\
1110 \\
1207 \\
1207\end{array}$ & $\begin{array}{l}7 / 09 / 84 \\
7 / 09 / 84 \\
7 / 25 / 84 \\
7 / 25 / 84 \\
8 / 24 / 84 \\
8 / 24 / 94 \\
8 / 27 / 84 \\
8 / 27 / 84\end{array}$ & $\begin{array}{l}915 \\
915 \\
910 \\
910 \\
845 \\
845 \\
845 \\
845\end{array}$ & $\begin{array}{l}- \\
- \\
-\end{array}$ & $\overline{-}$ \\
\hline $\begin{array}{l}\text { BLANKS } \\
\begin{array}{c}\text { VESSEL } \\
+ \\
j-13 \\
+ \\
\text { TUFF }\end{array}\end{array}$ & $\begin{array}{ll}G & 107 \\
G & 108 \\
\text { C } & 105 \\
\text { C } & 106 \\
G & 109 \\
G & 110 \\
G & 111 \\
G & 112\end{array}$ & $\begin{array}{l}7 \\
7 \\
14 \\
14 \\
28 \\
28 \\
56 \\
56\end{array}$ & $\begin{array}{l}= \\
- \\
- \\
=\end{array}$ & $\begin{array}{l}51 \\
56 \\
55 \\
56 \\
51 \\
56 \\
27 \\
28\end{array}$ & $\begin{array}{l}7 / 02 / 84 \\
7 / 02 / 84 \\
7 / 11 / 84 \\
7 / 11 / 84 \\
7 / 27 / 84 \\
7 / 27 / 84 \\
7 / 02 / 84 \\
7 / 02 / 84\end{array}$ & $\begin{array}{l}1207 \\
1207 \\
1130 \\
1130 \\
1110 \\
1110 \\
1207 \\
1207\end{array}$ & $\begin{array}{l}7 / 09 / 84 \\
7 / 09 / 84 \\
7 / 25 / 84 \\
7 / 25 / 84 \\
8 / 24 / 84 \\
8 / 24 / 84 \\
8 / 27 / 84 \\
8 / 27 / 84\end{array}$ & $\begin{array}{l}915 \\
+915 \\
+910 \\
910 \\
845 \\
845 \\
845 \\
845\end{array}$ & $\begin{array}{l}\text { - } \\
\text { - } \\
\text { - } \\
\text { - }\end{array}$ & $\begin{array}{l}- \\
- \\
- \\
- \\
- \\
-\end{array}$ \\
\hline
\end{tabular}


Table A-3c (Cont'd)

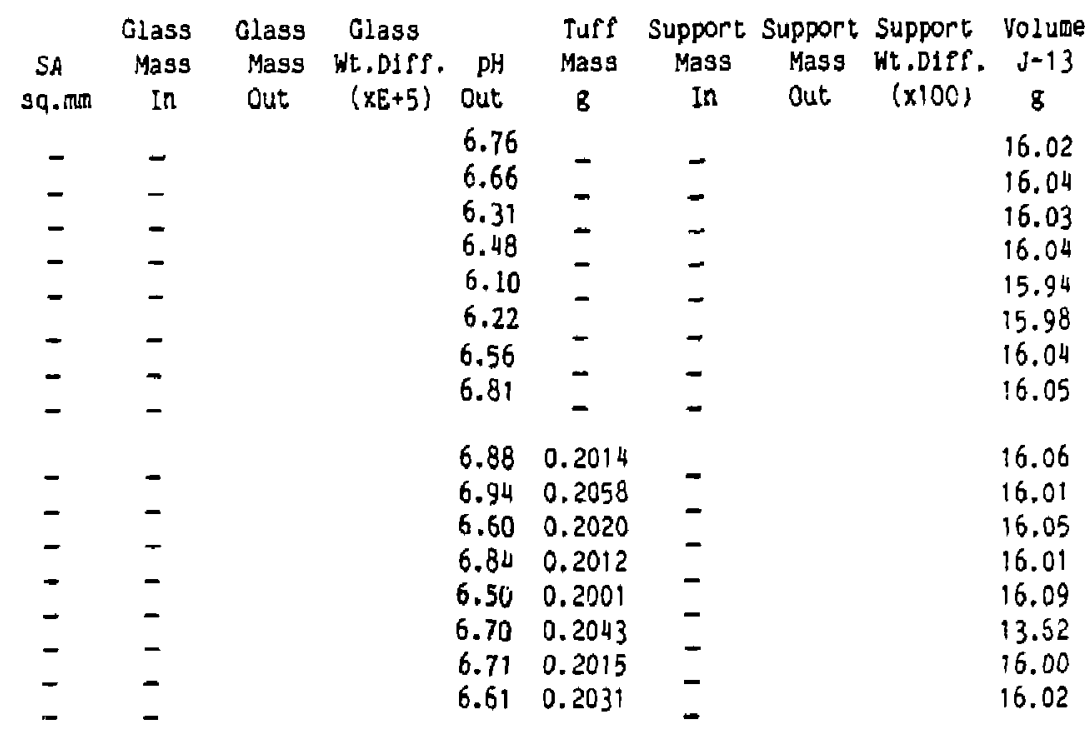


Table A-3c (Cont'd)

\begin{tabular}{cccc} 
Complete & \multicolumn{4}{c}{ Complete } & Assembly & LPE \\
Assemb1y & Assembly & Wt.Diff. \\
In & Out & $(\times 100)$ & wt \\
& & & \\
128.65 & 128.65 & 0 & 11.74 \\
129.47 & 129.46 & -1 & 11.78 \\
130.50 & 130.51 & +1 & 11.94 \\
129.57 & 129.55 & -2 & 11.96 \\
128.57 & 128.56 & -1 & 10.70 \\
129.44 & 129.40 & -4 & 12.16 \\
128.39 & 128.34 & -5 & 13.72 \\
129.46 & 129.38 & -8 & 13.75 \\
& & & \\
139.92 & 130.92 & 0 & 10.70 \\
130.29 & 130.25 & 0 & 11.74 \\
130.62 & 130.60 & -2 & 11.35 \\
130.29 & 130.27 & -2 & 11.48 \\
131.02 & 131.00 & -2 & 11.87 \\
127.90 & 127.89 & -1 & 8.86 \\
128.48 & 128.45 & -3 & 12.84 \\
129.12 & 129.08 & -4 & 12.62
\end{tabular}


TABLE A-4

FY 1986 Gamma Irradiation Experiments Matrix

This Table presents the matrix and selected results for the $1 R$ experiments. Included in this matrix are OR blank experiments done in conjunction with the $\mathbb{R}$ experiments. OR blank experiments were also done in conjunction $w 1$ th the $O R$ experiments. The difference between the two sets of blank experiments is the experiments listed in this Table used silicone rubber gaskets whereas the other blanks used Teflon gaskets. LPE refers to the weight of the sample submitted for cation analysis. 
Table A-4. FY 1986 Gamma Irradiation Experiments Matrix

\begin{tabular}{|c|c|c|c|c|c|c|c|c|c|c|c|c|}
\hline TYPE & $\begin{array}{l}\text { EXP'T } \\
\text { EXTHER }\end{array}$ & $\begin{array}{l}\text { SAYPLE } \\
\text { MUMBER }\end{array}$ & $\begin{array}{l}\text { EXP'T } \\
\text { DURATION } \\
\text { (deyx) }\end{array}$ & $\operatorname{DATE}_{\mathbf{I N}}$ & OATE & $\begin{array}{l}\text { VESSEL } \\
\text { MUMAGER }\end{array}$ & $\begin{array}{c}\text { CLASS } \\
\text { mickpss } \\
(\mathrm{mm})\end{array}$ & $\begin{array}{l}\text { DIRANST } \\
\text { (m) TER }\end{array}$ & 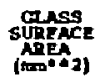 & 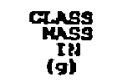 & $\begin{array}{l}\text { quss } \\
\text { ofst } \\
(g)\end{array}$ & 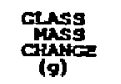 \\
\hline ATH-1C & $\begin{array}{l}G-390 \\
G-399 \\
G-390 \\
G-391 \\
G-392 \\
C-393 \\
G-394 \\
G-395 \\
C-396 \\
G-397\end{array}$ & 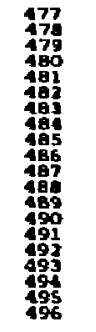 & $\begin{array}{r}28 \\
28 \\
56 \\
56 \\
91 \\
91 \\
182 \\
182 \\
280 \\
280\end{array}$ & $\begin{array}{c}7 / 24 \\
7 / 24 \\
7 / 8 \\
7 / 6 \\
12 / 10 \\
12 / 10 \\
12 / 10 \\
12 / 10 \\
12 / 10 \\
12 / 10\end{array}$ & $\begin{array}{l}9 / 21 \\
9 / 21 \\
9 / 2 \\
9 / 2 \\
3 / 19 \\
3 / 19 \\
6 / 10 \\
6 / 10 \\
9 / 24 \\
9 / 24\end{array}$ & $\begin{array}{r}10 \\
107 \\
147 \\
52 \\
74 \\
76 \\
60 \\
70 \\
71 \\
75\end{array}$ & 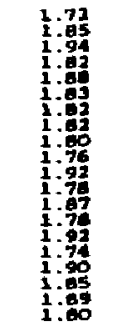 & $\begin{array}{l}10: 73 \\
10: 72 \\
10: 95 \\
10: 69 \\
10: \frac{11}{10} 7 \\
10: 71 \\
10: 71 \\
10: 91 \\
10: 71 \\
10: 75 \\
10: 71 \\
10: 77 \\
10: 70 \\
10: 73 \\
10: 73 \\
10: 74 \\
10: 71\end{array}$ & 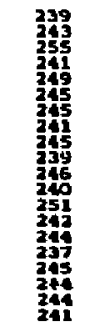 & 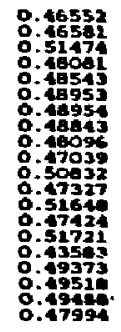 & 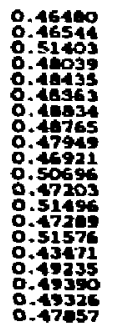 & 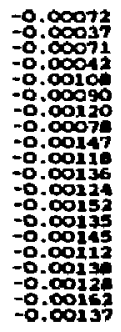 \\
\hline ATM-1e * & $\begin{array}{l}G-399 \\
G-399 \\
G-400 \\
G-401 \\
c-402 \\
G-403 \\
G-404 \\
G-405 \\
G-406 \\
G-407\end{array}$ & $\begin{array}{l}197 \\
197 \\
199 \\
500 \\
501 \\
505 \\
503 \\
504 \\
505 \\
506 \\
507 \\
500 \\
509 \\
510 \\
511 \\
512 \\
513 \\
514 \\
515 \\
516\end{array}$ & $\begin{array}{l}20 \\
28 \\
56 \\
56 \\
91 \\
91 \\
1 E 2 \\
1=2 \\
280 \\
280\end{array}$ & $\begin{array}{c}7 / 24 \\
7 / 24 \\
7 / 8 \\
7 / 8 \\
12 / 18 \\
12 / 10 \\
12 / 10 \\
12 / 10 \\
12 / 10 \\
12 / 10\end{array}$ & $\begin{array}{l}9 / 21 \\
9 / 21 \\
9 / 2 \\
9 / 2 \\
3 / 19 \\
3 / 19 \\
6 / 19 \\
6 / 10 \\
9 / 24 \\
9 / 24\end{array}$ & $\begin{array}{r}122 \\
106 \\
134 \\
39 \\
90 \\
77 \\
70 \\
08 \\
01 \\
82\end{array}$ & 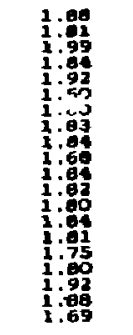 & 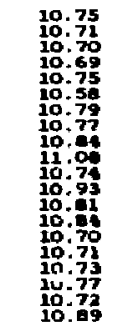 & 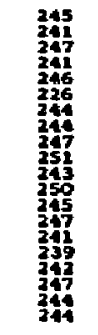 & 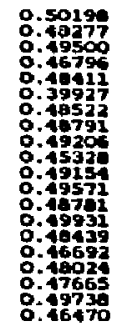 & 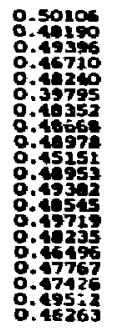 & 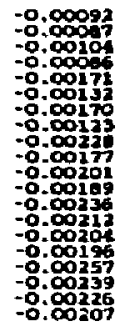 \\
\hline ADM-A & $\begin{array}{l}C-409 \\
G-409 \\
G-410\end{array}$ & $\begin{array}{l}527 \\
518 \\
519 \\
530 \\
572 \\
522\end{array}$ & $\begin{array}{l}28 \\
28 \\
56\end{array}$ & $\begin{array}{l}7 / 24 \\
7 / 24 \\
7 / 6\end{array}$ & $\begin{array}{l}a / 21 \\
0 / 21 \\
9 / 2\end{array}$ & $\begin{array}{r}127 \\
166 \\
77\end{array}$ & $\begin{array}{l}1.79 \\
1: 79 \\
1: 76 \\
1: 78 \\
2.1 .2 \\
1.94\end{array}$ & $\begin{array}{l}10.92 \\
10: 83 \\
10.02 \\
10: 94 \\
10: 02 \\
10.83\end{array}$ & $\begin{array}{l}245 \\
345 \\
344 \\
240 \\
253 \\
252\end{array}$ & 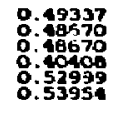 & $\begin{array}{l}0.49312 \\
0: 40640 \\
0: 49477 \\
0.40374 \\
0.52922 \\
0.53879\end{array}$ & $\begin{array}{r}-0.00025 \\
-0.00030 \\
0.00757 \\
-0.00034 \\
-0.00077 \\
-0.00075\end{array}$ \\
\hline
\end{tabular}


Table A-4 (Cont'd)

\begin{tabular}{|c|c|c|c|c|c|c|c|c|c|c|c|c|}
\hline EXP'T & EXP'T & $\begin{array}{l}\text { NUPDER } \\
\text { NOABER }\end{array}$ & 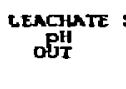 & 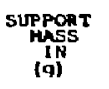 & $\begin{array}{c}\text { suppont } \\
\text { puss } \\
\text { out } \\
\text { (g) }\end{array}$ & 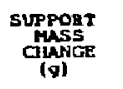 & 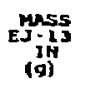 & $\begin{array}{c}\text { ASSEHALX } \\
\text { MLSS } \\
\text { (a) }\end{array}$ & $\begin{array}{l}\text { Assemplx } \\
\text { ouss } \\
\text { (9) }\end{array}$ & 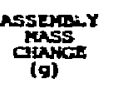 & 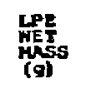 & 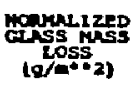 \\
\hline \multirow[t]{10}{*}{ Ant-1c } & C- 3es & & 0.84 & 4.9439 & 4.9438 & -0.0001 & 14.69 & 303.15 & 303.10 & -0.05 & 22.28 & 2.26 \\
\hline & C-389 & & 0.92 & 4.9266 & 4.9265 & $-0 . \infty 001$ & 14.90 & 300.92 & 300.40 & -0.02 & 12.35 & 2.20 \\
\hline & G-390 & 8 & 6.12 & 4.0950 & 4.9854 & 0.0904 & 14.47 & 303.10 & 303.12 & -0.06 & 12.26 & 4.02 \\
\hline & $0-391$ & & c. 30 & 1.9615 & 4.9017 & 0.0002 & 14.90 & 302.31 & 302.35 & -0.03 & 13.30 & 4.07 \\
\hline & C- 392 & & 7.95 & 4.9190 & +.9195 & -0.0003 & 14.91 & 305.05 & 304.90 & $=0.07$ & 12.70 & 5.47 \\
\hline & C-393 & & 1.08 & 4.0923 & 4.0926 & -0.0002 & 14.en & 300.49 & 300.02 & -0.07 & 22.63 & 5.34 \\
\hline & G-394 & & 7.96 & 1. 18649 & 4.0649 & 0.0000 & 14.49 & 300.99 & 300.14 & -0.05 & 12.24 & 5.63 \\
\hline & $c .395$ & & 7.81 & 4.9339 & 4.9336 & -0.0003 & 14.69 & 302.62 & 302.44 & -0.17 & 12.13 & 3.4 \\
\hline & c-336 & 49 & 7.05 & 4.9300 & 4.9299 & -0.0001 & 14.92 & 303.02 & 303.63 & -0.19 & 12.20 & $3.4 t$ \\
\hline & 6.397 & & 7.72 & 4.9370 & 4.9373 & 0.0002 & 14.91 & 300.62 & 300.52 & -0.10 & 11.99 & 6.17 \\
\hline \multirow{10}{*}{$\begin{array}{c}\text { ATM-1C } \\
\text { ruEE }\end{array}$} & G-398 & 197 & 0.42 & 4.9973 & 4.0973 & 0.0000 & $24+63$ & 304.29 & 304.10 & -0.01 & 21.95 & 3.60 \\
\hline & $c-399$ & & 0.22 & 4.0517 & as16 & -0.0001 & 24.59 & 303.44 & 301.41 & -0.03 & 11.12 & 5.91 \\
\hline & $c-400$ & & 0.20 & 5.1235 & 5.0235 & -0.1000 & 14.49 & 302.61 & 302.55 & -0.06 & 11.72 & 6.12 \\
\hline & $c-\infty 01$ & 50 & 0.20 & 4.8916 & 1.6916 & 0.0000 & 14.49 & 304.09 & 304.04 & -0.05 & 11.70 & 6.00 \\
\hline & $c-402$ & 5 & 8.09 & 4.9645 & 4. $=601$ & -0.0004 & 14.62 & 304.30 & 304.30 & -0.00 & 12.26 & 0.12 \\
\hline & $c-\infty 03$ & 507 & 8. 24 & 4.9603 & 4.9603 & 0.0000 & 14.57 & 304.50 & 304.46 & -0.06 & 12.27 & 1.50 \\
\hline & C-404 & & $\Delta .22$ & 4.9905 & 1.9904 & -0.0001 & 14.60 & 306.71 & 306.68 & -0.03 & 21.09 & 9.11 \\
\hline & $0-405$ & 51 & 7.80 & 4.6719 & 4.9718 & -0.0001 & 14.50 & 305.51 & 305.41 & -0.10 & 12.33 & 0.34 \\
\hline & $0-406$ & & 7.97 & 4.9480 & 1.9530 & 0.0100 & $=4.50$ & 309.30 & 309.17 & -0.13 & 11.12 & 10.15 \\
\hline & C-407 & 726 & 6.86 & 4,9562 & 4.9460 & -0.0101 & 24.59 & 300.89 & 300.75 & -0.14 & 11.99 & 6.87 \\
\hline \multirow[t]{3}{*}{ ATM-B } & C-406 & 577 & 8.67 & 4.9383 & 1.8460 & -0.0915 & 14.94 & 307.52 & 307.40 & -0.04 & 11.99 & 1.10 \\
\hline & & & B. 54 & 4.8626 & 4.9130 & 0.0512 & 14.91 & 104.31 & 304.27 & -0.04 & 12.20 & 1.41 \\
\hline & G-410 & 322 & 7.92 & 48970 & 4.8972 & 0.0002 & 14.60 & 302,30 & 307.24 & -0.06 & 11.72 & 3.01 \\
\hline
\end{tabular}


Table A-4 (Cont'd)

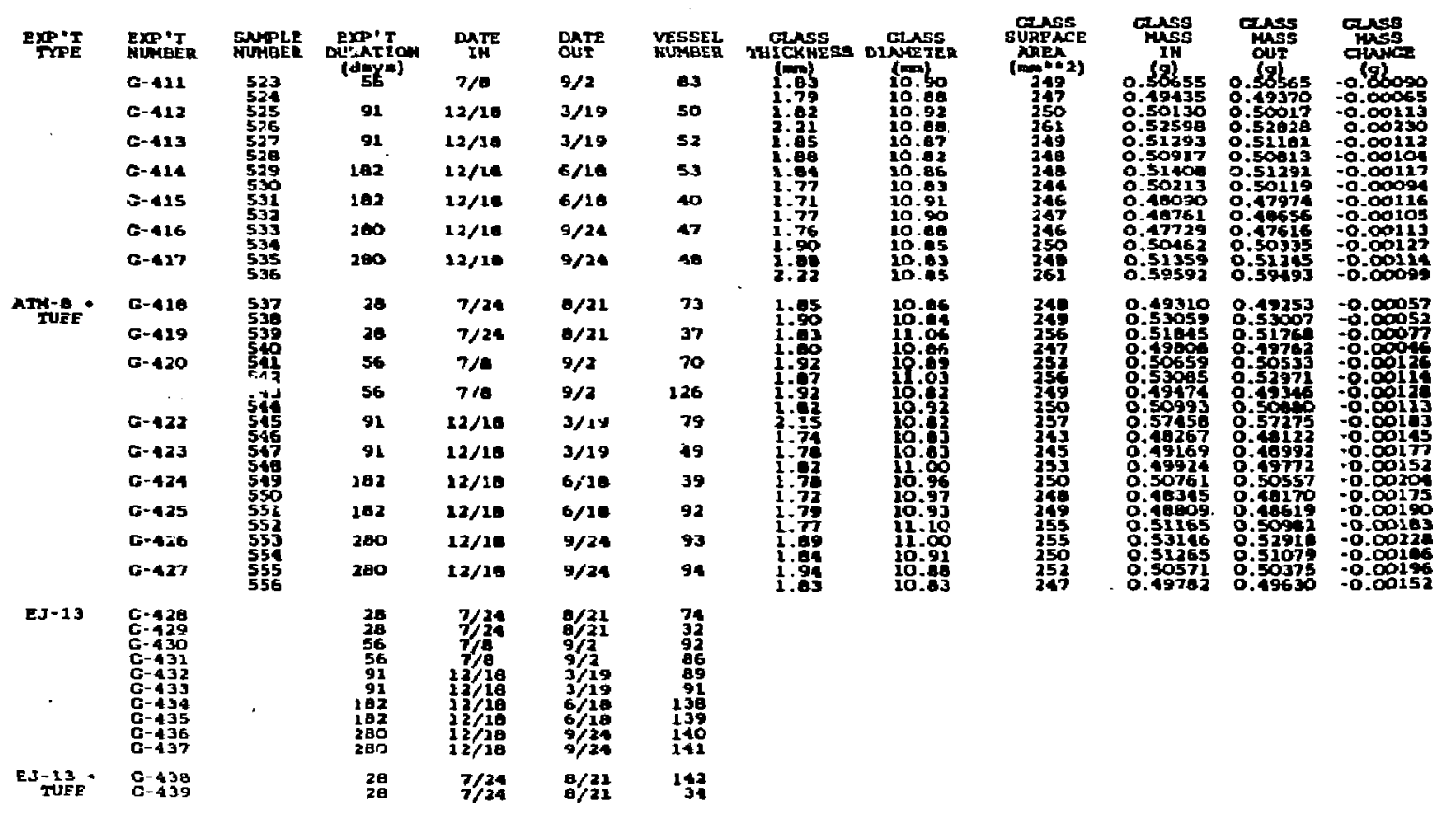


Table A-4 (Cont'd)

\begin{tabular}{|c|c|c|c|c|c|c|c|c|c|c|c|c|}
\hline \multirow[t]{8}{*}{ EXP'T } & EXP'T & SAYPTE.E & $\begin{array}{l}\text { LEACFATE } \\
\text { Ob! }\end{array}$ & $\begin{array}{c}\text { Suppops } \\
\text { Miss } \\
\text { 19i }\end{array}$ & $\begin{array}{c}\text { suppont } \\
\text { wis } \\
\text { our } \\
\text { (g) }\end{array}$ & 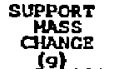 & 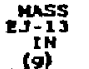 & 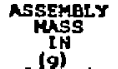 & $\begin{array}{c}\text { ASSEmbL } \\
\text { MSSS } \\
\text { Out } \\
\text { (q) }\end{array}$ & $\begin{array}{l}\text { NSSEMELY } \\
\text { CHSS } \\
\text { COME }\end{array}$ & $\begin{array}{l}\text { LPE } \\
\text { MEts } \\
\text { Mg) }\end{array}$ & 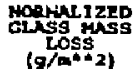 \\
\hline & C-111 & $\begin{array}{l}523 \\
524\end{array}$ & 7.05 & sa & 5.8158 & & & $3 d 2.12$ & 982.07 & & & \\
\hline & $6-412$ & $\begin{array}{l}525 \\
526\end{array}$ & 7.61 & 4.9150 & .9150 & 0.0000 & 14.09 & 308.50 & 300.40 & -0.10 & 11.99 & 4.42 \\
\hline & $0-413$ & 527 & 7.68 & 4.9280 & 4.9186 & -0.0002 & 14.92 & 302.45 & $3 n 3.30$ & -0.07 & 12.37 & 4.35 \\
\hline & G-414 & 529 & 7.70 & 4.9130 & 4.9226 & -0.0004 & 14.06 & 303.20 & 303.12 & -0.26 & $11 . \infty$ & 4.20 \\
\hline & G-425 & $\begin{array}{l}531 \\
532\end{array}$ & 7.54 & 4.9192 & 4.9187 & -0.0006 & 14.97 & 303.94 & 303.79 & -0.15 & 12.21 & 4.48 \\
\hline & G-416 & $\begin{array}{l}\mathbf{5 3 3} \\
\mathbf{5 3 4}\end{array}$ & 7.78 & 4.5045 & 4.0043 & -0.0002 & 14.07 & 301.01 & 301.63 & -0.10 & 11.67 & 4.86 \\
\hline & C-417 & $\begin{array}{l}\mathbf{5} 35 \\
\mathbf{5 3 6}\end{array}$ & 5.82 & 4.9144 & 4.9131 & -0.0013 & 16.09 & 303.45 & 303.17 & -0.20 & 12.72 & $\$ .19$ \\
\hline \multirow{10}{*}{ ATMFE } & $0-418$ & $\begin{array}{l}537 \\
536\end{array}$ & $=.36$ & 4.9220 & 4.9464 & 0.0244 & 14.61 & 310.76 & 310.70 & -0.06 & 11.4 & 2.19 \\
\hline & $6-419$ & & $\bullet .05$ & 5.0393 & 4.9213 & -0.1280 & 24.62 & 300.66 & 304.14 & -0.01 & 12.07 & 2.45 \\
\hline & C.426) & & 7.59 & 1.0762 & 4.8750 & -0.0006 & 24.47 & 305.84 & 305.77 & -0.07 & 21.05 & 1.73 \\
\hline & $(6-42)$ & & 7.40 & 5,0126 & 50121 & -0.0005 & 14.52 & 304.12 & 304.04 & -0.07 & 1.10 & 4.93 \\
\hline & $c-422$ & 54 & 7.95 & 1.8971 & 1.091 .7 & -0.0007 & 14.63 & 312.39 & 312.32 & -0.00 & 21.46 & 6.55 \\
\hline & G-423 & & 7.70 & 4.9677 & 4.9270 & -0.0399 & 14.65 & 311.21 & 311.12 & -0.09 & 11.66 & 6.61 \\
\hline & $0-424$ & & 7.52 & 4.9ra7 & 4.9606 & $-0 . \infty \infty 01$ & 24.59 & 304.22 & 304.13 & -0.09 & 11.30 & 7.61 \\
\hline & G-425 & 551 & 7.74 & $4.8 r ? 6$ & 4.8723 & -0.0003 & 14.64 & sou. 68 & 35.76 & -0.14 & 11.00 & 7.40 \\
\hline & G- -26 & 553 & B.or & 4.0296 & 4.8297 & 0.0001 & 14.50 & 305.92 & 305.60 & -0.12 & 11.29 & 0.19 \\
\hline & $0-427$ & $\begin{array}{l}555 \\
556\end{array}$ & 7.92 & 1.9542 & 4.9537 & -0.0004 & 14.59 & 312.52 & 312.32 & -0.20 & 11.60 & 6.90 \\
\hline$E \mathbf{J}-13$ & $\begin{array}{l}G-428 \\
G-429 \\
G-430 \\
G-431 \\
G-432 \\
G-433 \\
G-434 \\
6-435 \\
6-436 \\
6-437\end{array}$ & ' & $\begin{array}{l}7.14 \\
7: 39 \\
6: 57 \\
6: 75 \\
6: 26 \\
5: 54 \\
6: 51 \\
6.99 \\
6.91 \\
6.98\end{array}$ & & & & $\begin{array}{l}15.49 \\
15: 17 \\
16: 20 \\
16: 20 \\
16: 19 \\
16: 19 \\
16: 22 \\
16: 16 \\
16: 19\end{array}$ & 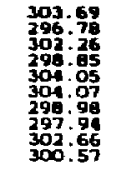 & 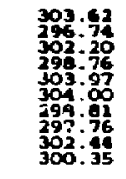 & $\begin{array}{l}-0.07 \\
-0.07 \\
=0.06 \\
=0.09 \\
=0.09 \\
-0.07 \\
=-0.17 \\
=0.10 \\
-0.22 \\
-0.22 \\
0.00\end{array}$ & $\begin{array}{l}13: 49 \\
12: 91 \\
13: 93 \\
13: 83 \\
14: 03 \\
13.66 \\
13: 70 \\
13: 63 \\
33: 72\end{array}$ & \\
\hline $\begin{array}{l}\text { EJ-13 } \\
\text { JUFE* }\end{array}$ & $\begin{array}{c}G-438 \\
6-439\end{array}$ & & $7: 03$ & & & & $\begin{array}{l}15.53 \\
15.51\end{array}$ & $\begin{array}{l}299.94 \\
305: 26\end{array}$ & $\begin{array}{l}299: \infty \\
325\end{array}$ & $=0.94$ & $\begin{array}{l}13 . \infty \\
13 . \infty\end{array}$ & \\
\hline
\end{tabular}


Table A-4 (Con' 'd)

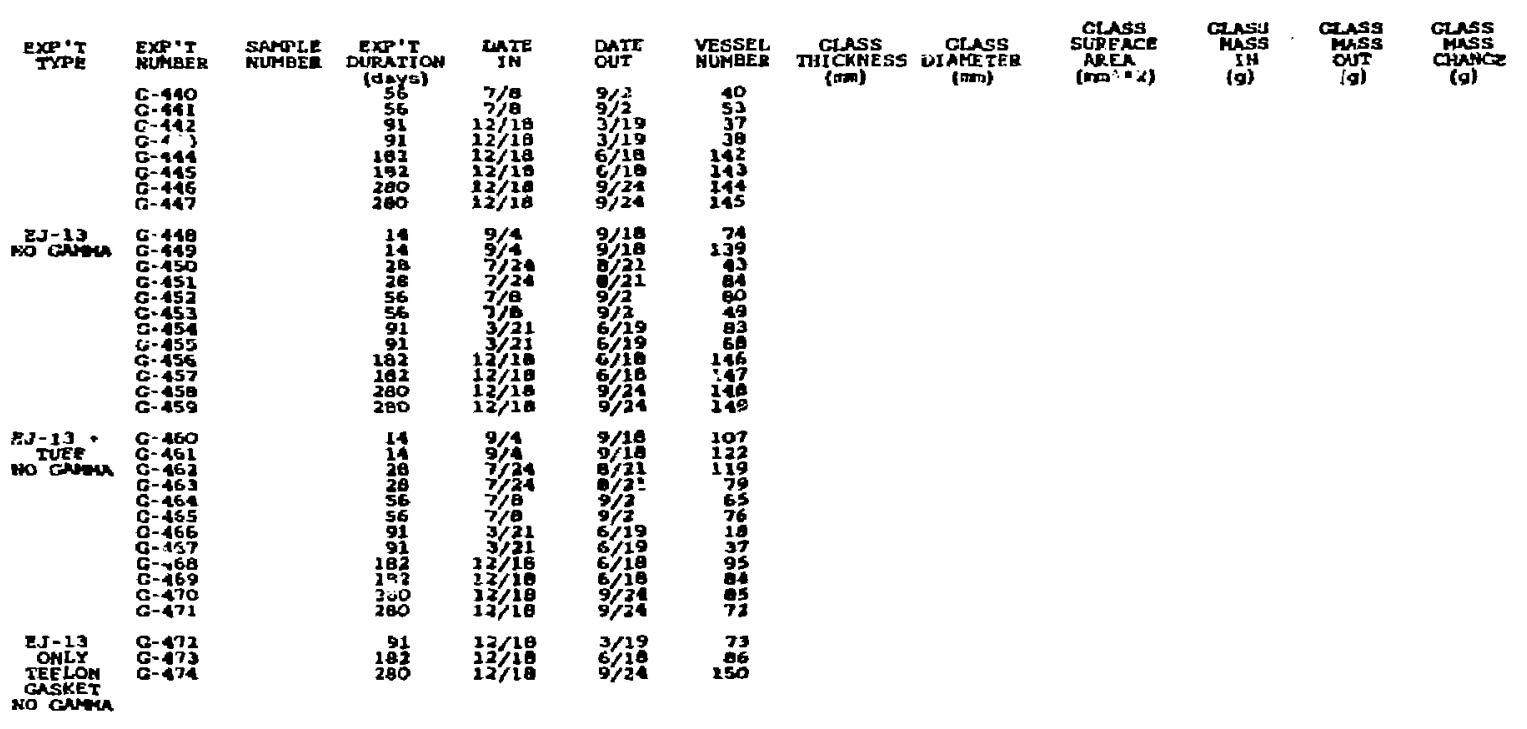


Table A-4 (Cont'd)

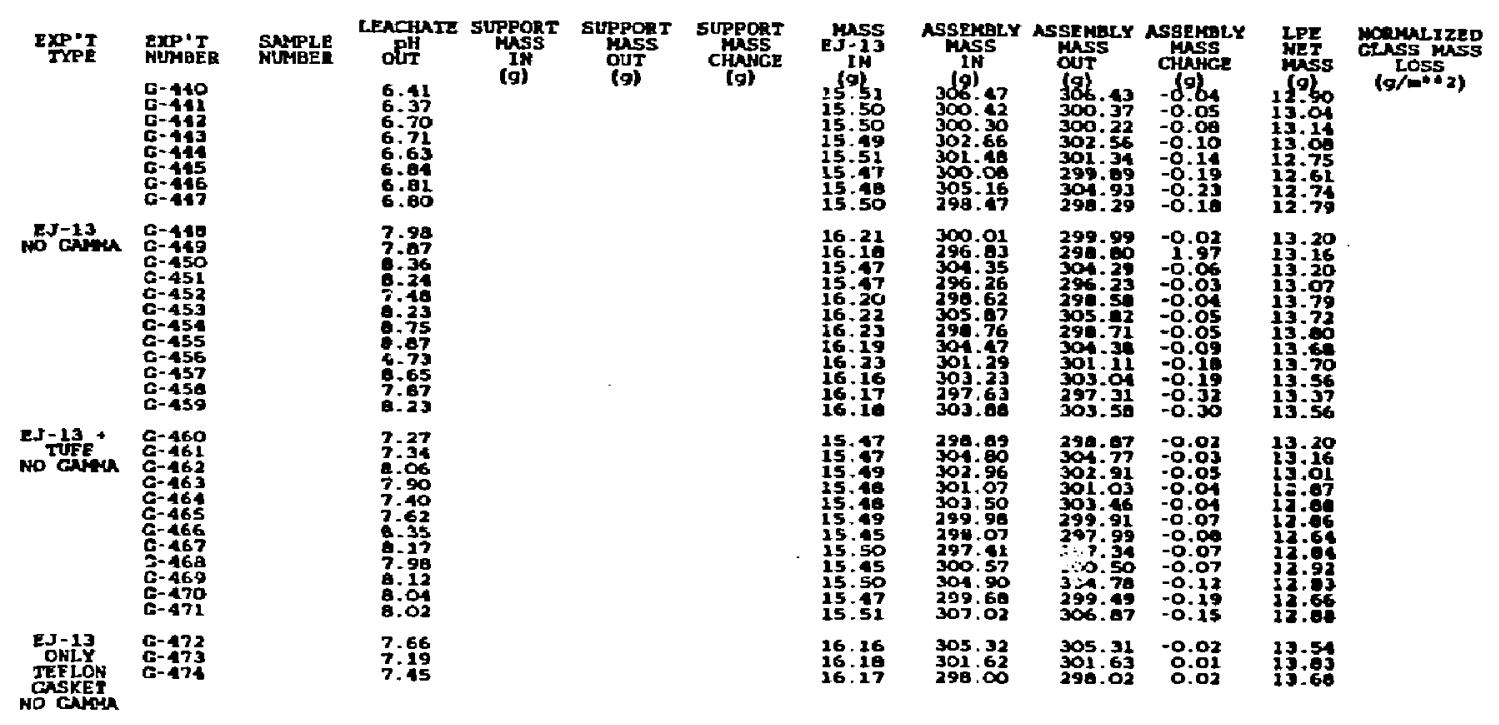


TABLE $A-5 a$

Experimental Matrix and Component Weights for the $O R$ (Teflon) Gasket Experiments

This Table presents the matrix and selected results from the $O R$ experiments done with Teflon gaskets. 
Table A-5a. Experimental Matrix and Component Weights for the OR (Tefion) Gasket Experiments

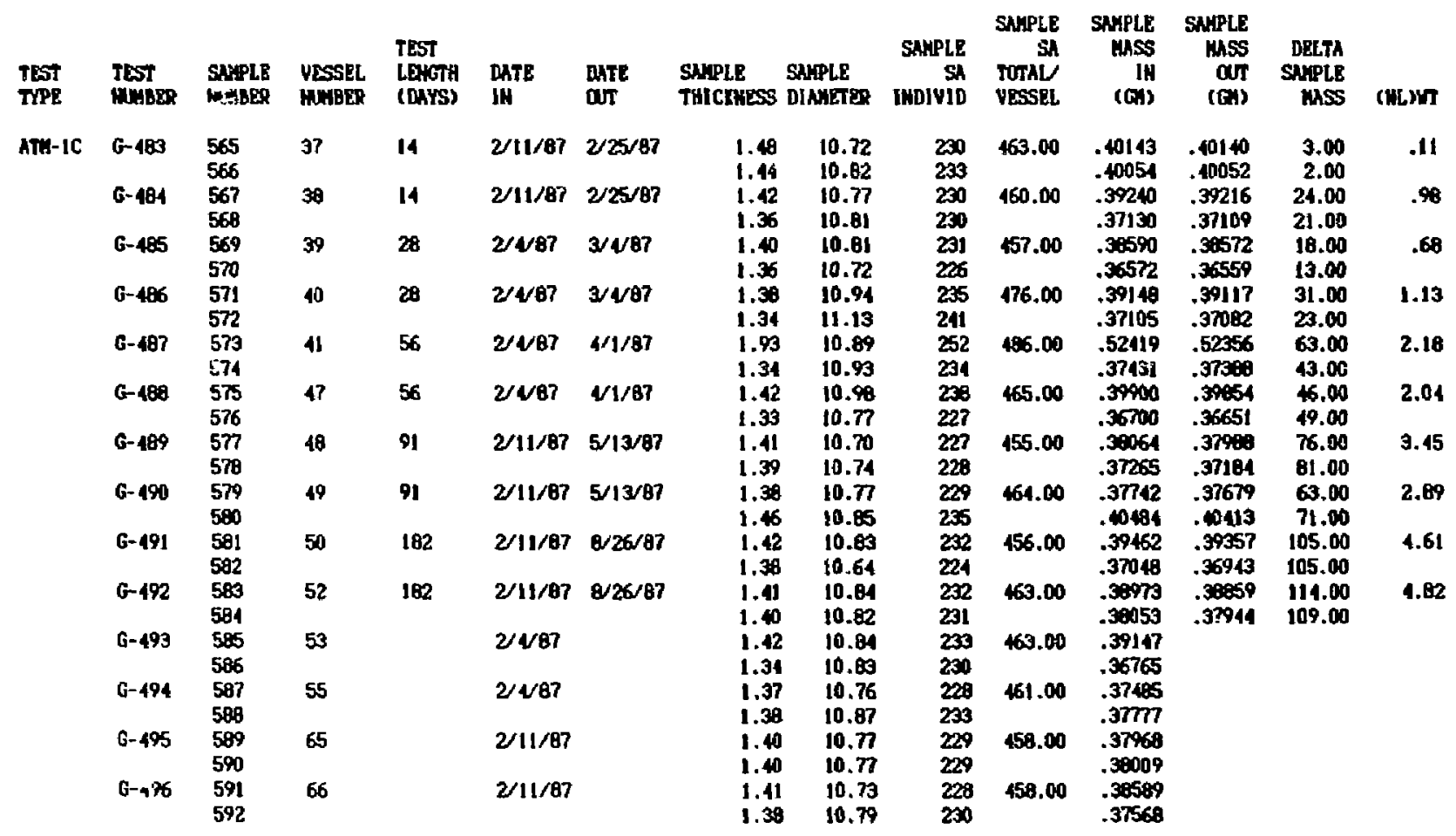


Table A-5a (Cont'd)

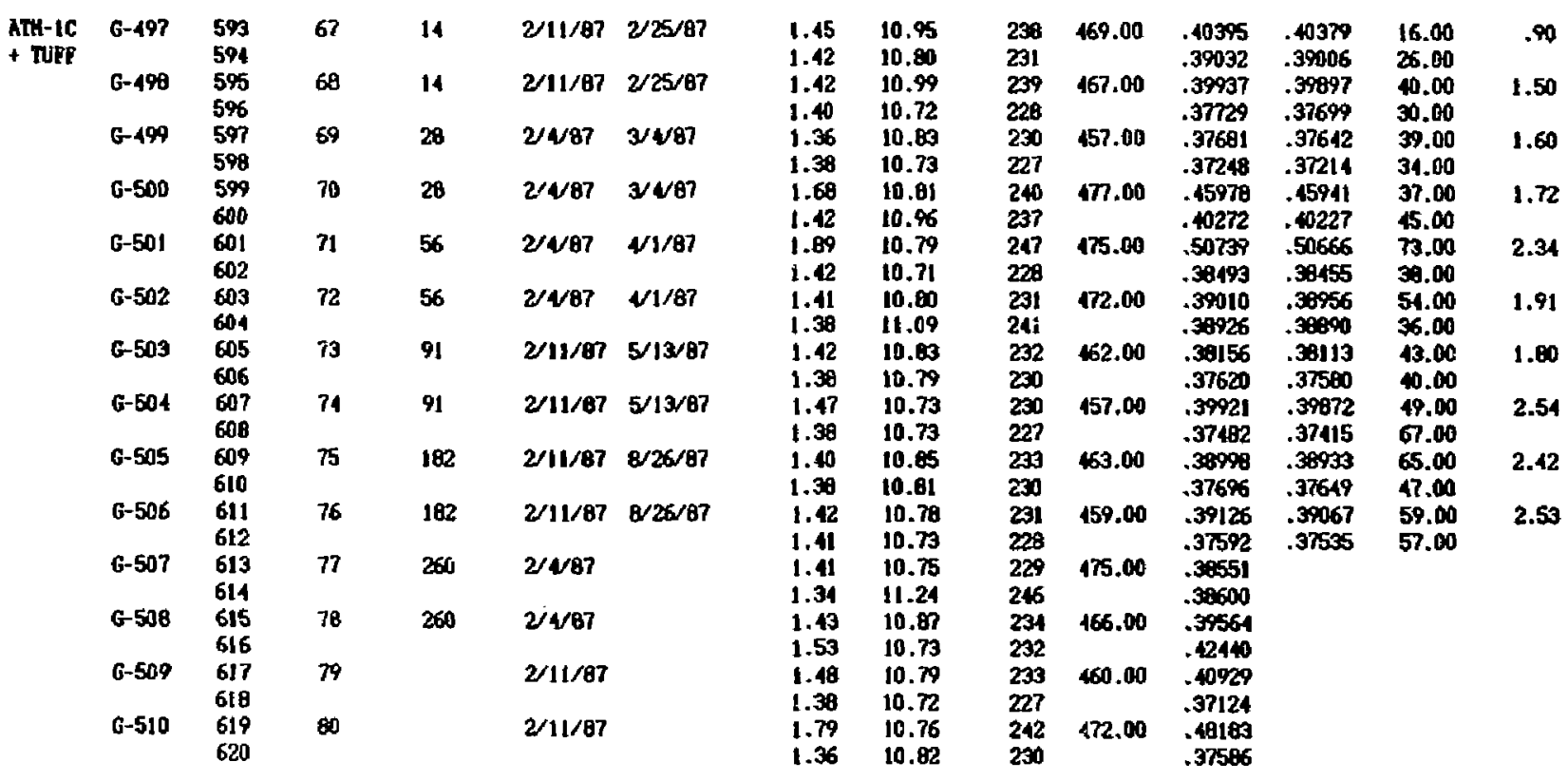


Table A-5a (Cont'd)

\begin{tabular}{|c|c|c|c|c|c|c|c|c|c|c|c|c|c|c|}
\hline \multirow{17}{*}{$A T H-B C$} & G-5.1 & 621 & 61 & 14 & $2 / 11 / 87$ & $2 / 25 / 87$ & 1.59 & 10.88 & 240 & 405.00 & .44679 & .14870 & 9.00 & .25 \\
\hline & & 622 & & & & & 1.70 & 10.91 & 245 & & .17105 & .47182 & 3.00 & \\
\hline & $6-512$ & $\begin{array}{l}623 \\
624\end{array}$ & 82 & 14 & $2 / 11 / 87$ & $2 / 25 / 67$ & $\begin{array}{l}1.58 \\
1.58\end{array}$ & $\begin{array}{l}10.83 \\
10.87\end{array}$ & $\begin{array}{l}239 \\
239\end{array}$ & 177.00 & $\begin{array}{l}.44662 \\
.43100\end{array}$ & $\begin{array}{l}.44664 \\
.43109\end{array}$ & $\begin{array}{l}-2.00 \\
-1.00\end{array}$ & -.06 \\
\hline & G-513 & $\begin{array}{l}625 \\
626\end{array}$ & 63 & 28 & $2 / 4 / 87$ & $3 / 4 / 67$ & $\begin{array}{l}1.36 \\
1.50\end{array}$ & $\begin{array}{l}10.89 \\
10.67\end{array}$ & $\begin{array}{l}233 \\
237\end{array}$ & 170.00 & $\begin{array}{l}.30016 \\
.42254\end{array}$ & $\begin{array}{l}.38015 \\
.42244\end{array}$ & $\begin{array}{r}1.00 \\
10.00\end{array}$ & .29 \\
\hline & G-514 & 627 & 84 & $2 \theta$ & $2 / 4 / 87$ & $3 / 4 / 87$ & 1.55 & 10.93 & 241 & 492.00 & .43122 & .43126 & -4.00 & .02 \\
\hline & G-515 & $\begin{array}{l}628 \\
629\end{array}$ & 85 & 56 & $2 / 4 / 87$ & $t / 1 / 87$ & $\begin{array}{l}1.68 \\
1.45\end{array}$ & $\begin{array}{l}11.07 \\
10.89\end{array}$ & $\begin{array}{l}251 \\
236\end{array}$ & 472.00 & $\begin{array}{r}.46546 \\
.40278\end{array}$ & $\begin{array}{l}.46541 \\
.40255\end{array}$ & $\begin{array}{r}5.00 \\
23.00\end{array}$ & 1.14 \\
\hline & & 630 & & & & & 1.56 & 10.87 & 239 & & .42787 & .42756 & 31.00 & \\
\hline & G-516 & $\begin{array}{l}631 \\
632\end{array}$ & 86 & 56 & $2 / 4 / 07$ & $/ 1 / 87$ & $\begin{array}{l}1.71 \\
1.54\end{array}$ & $\begin{array}{l}11.05 \\
10.69\end{array}$ & $\begin{array}{l}251 \\
230\end{array}$ & 469.00 & $\begin{array}{l}.48761 \\
.42508\end{array}$ & $\begin{array}{r}.49739 \\
.12476\end{array}$ & $\begin{array}{r}22.00 \\
32.00\end{array}$ & 1.10 \\
\hline & G-517 & $\begin{array}{l}633 \\
694\end{array}$ & 87 & 91 & $2 / 11 / \theta ?$ & $5 / 13 / 87$ & $\begin{array}{l}1.45 \\
1.82\end{array}$ & $\begin{array}{l}10.95 \\
10.02\end{array}$ & $\begin{array}{l}239 \\
246\end{array}$ & 491.00 & $\begin{array}{r}11169 \\
.50706\end{array}$ & $\begin{array}{l}.41143 \\
.54679\end{array}$ & $\begin{array}{l}25.00 \\
77.00\end{array}$ & 1.10 \\
\hline & G-518 & $\begin{array}{l}635 \\
636\end{array}$ & 88 & 91 & $2 / 11 / 27$ & $5 / 13 / 87$ & $\begin{array}{l}1.58 \\
1.45\end{array}$ & $\begin{array}{l}10.98 \\
10.86\end{array}$ & $\begin{array}{l}244 \\
235\end{array}$ & 479.00 & $\begin{array}{r}.14506 \\
.40335\end{array}$ & $\begin{array}{r}.44566 \\
.40309\end{array}$ & $\begin{array}{l}20.00 \\
26.00\end{array}$ & .96 \\
\hline & $6-519$ & 637 & $\theta$ & 182 & $2 / 11 / 87$ & $\mathrm{~B} / 26 / 87$ & 1.56 & 10.88 & 239 & $\$ 95.00$ & .42018 & .44051 & 30.00 & 1.42 \\
\hline & & 638 & & & & & $1.5 ?$ & 11.05 & 246 & & .42033 & .41994 & 39.80 & \\
\hline & G-520 & $\begin{array}{l}639 \\
640\end{array}$ & 91 & 182 & $2 / 11 / 67$ & $8 / 26 / 87$ & $\begin{array}{l}1.49 \\
1.64\end{array}$ & $\begin{array}{l}10.82 \\
10.85\end{array}$ & $\begin{array}{l}224 \\
241\end{array}$ & 475,00 & $\begin{array}{l}.39394 \\
.45910\end{array}$ & $\begin{array}{r}.39364 \\
.45972\end{array}$ & $\begin{array}{r}30.00 \\
39.00\end{array}$ & 1.12 \\
\hline & G-521 & $\begin{array}{l}641 \\
642\end{array}$ & 92 & 260 & $2 / 4 / 87$ & & $\begin{array}{l}1.33 \\
1.39\end{array}$ & $\begin{array}{l}10.86 \\
10.87\end{array}$ & $\begin{array}{l}231 \\
239\end{array}$ & 464.00 & $\begin{array}{l}.37646 \\
.388265\end{array}$ & & & \\
\hline & G-522 & $\begin{array}{l}613 \\
614\end{array}$ & 93 & 260 & $2 / 4 / \theta 7$ & & $\begin{array}{l}1.53 \\
1.34\end{array}$ & $\begin{array}{l}10.81 \\
10.06\end{array}$ & $\begin{array}{l}2335 \\
231\end{array}$ & $\$ 66.00$ & $\begin{array}{r}.42972 \\
.37269\end{array}$ & & & \\
\hline & G-523 & $\begin{array}{l}645 \\
646\end{array}$ & 94 & & $2 / 11 / 87$ & & $\begin{array}{l}1.56 \\
1.76\end{array}$ & $\begin{array}{l}10.67 \\
10.91\end{array}$ & $\begin{array}{l}239 \\
247\end{array}$ & 496.00 & $\begin{array}{l}.44679 \\
.44908\end{array}$ & & & \\
\hline & 6-524 & $\begin{array}{l}647 \\
648\end{array}$ & 95 & & $2 / 11 / 87$ & & $\begin{array}{l}1.55 \\
1.51\end{array}$ & $\begin{array}{l}11.00 \\
10.86\end{array}$ & $\begin{array}{l}214 \\
257\end{array}$ & 481.00 & $\begin{array}{l}.43757 \\
.34738\end{array}$ & & & \\
\hline
\end{tabular}


Table A-5a (Cont'd)

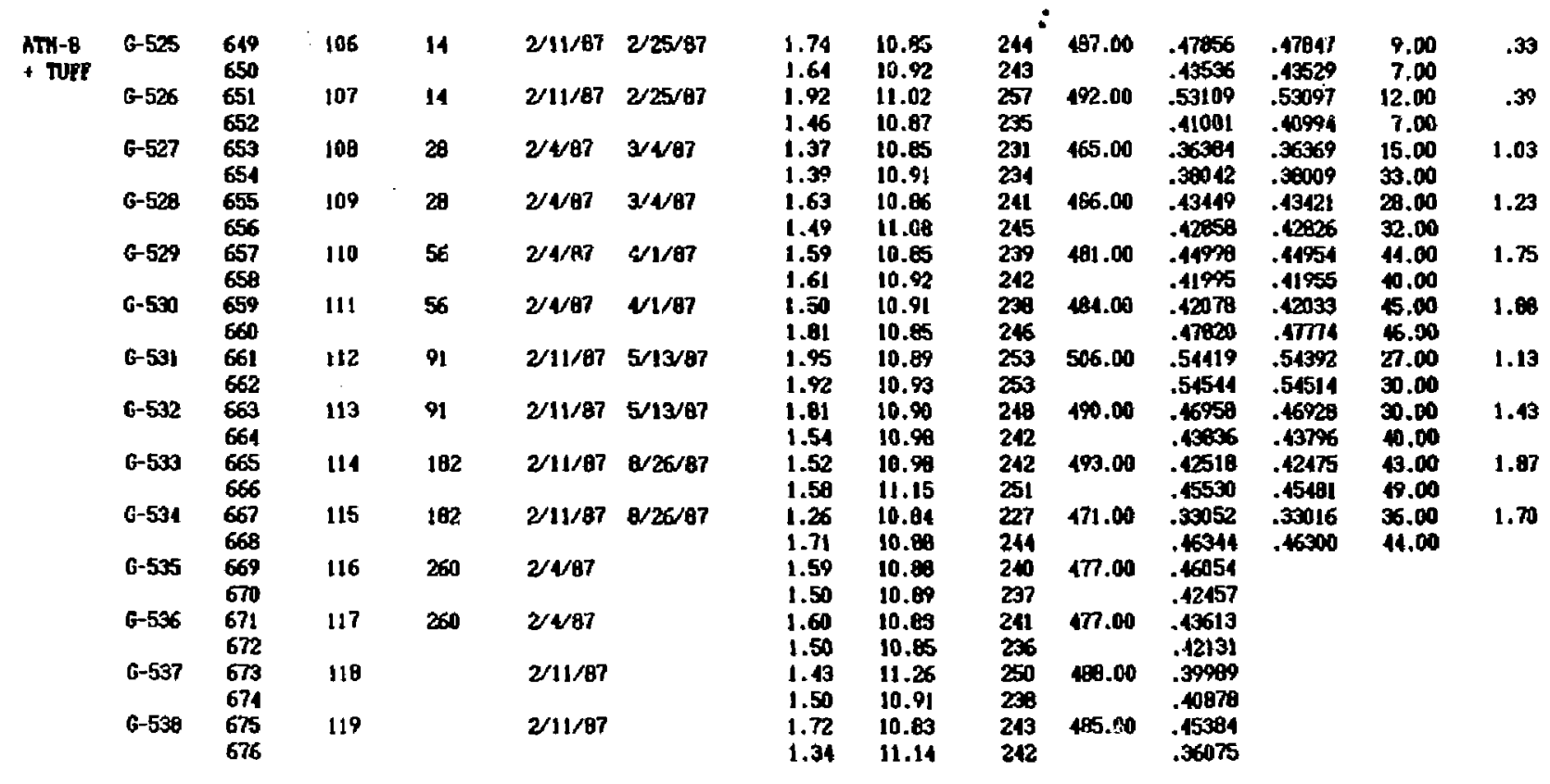


Table A-5a (Cont'd)

\begin{tabular}{|c|c|c|c|c|c|c|c|c|}
\hline $5 T T$ & IST & VESSEL & $\begin{array}{l}\text { TEST } \\
\text { LE!GTH } \\
\text { (LAYS) }\end{array}$ & $\begin{array}{l}\text { DATB } \\
\text { IH }\end{array}$ & $\begin{array}{l}\text { OATE } \\
\text { OUT }\end{array}$ & $\begin{array}{ll}\text { Hzo } & \text { C } \\
\text { Poudas } & A \\
\text { IH } & \text { I }\end{array}$ & $\begin{array}{l}\text { COAPLETE } \\
\text { ASSDUBLY } \\
\text { IN }\end{array}$ & $\begin{array}{l}\text { MPLETE } \\
\text { SDEBAYY } \\
T\end{array}$ \\
\hline $\begin{array}{l}\text { BLANR } \\
\text { EJ-13 } \\
\text { ONIY }\end{array}$ & $\begin{array}{l}6-559 \\
6-540 \\
6-541 \\
G-542 \\
G-543 \\
G-541 \\
G-545 \\
6-546 \\
G-547 \\
G-548 \\
G-549 \\
G-550 \\
G-551 \\
G-552\end{array}$ & $\begin{array}{l}120 \\
121 \\
122 \\
123 \\
124 \\
125 \\
126 \\
127 \\
128 \\
129 \\
130 \\
131 \\
132 \\
139\end{array}$ & $\begin{array}{l}14 \\
14 \\
28 \\
28 \\
56 \\
56 \\
91 \\
91 \\
182 \\
182 \\
260 \\
260 \\
\text { Lof } \\
\text { La146 }\end{array}$ & $\begin{array}{l}2 / 11 / 87 \\
2 / 11 / 67 \\
2 / 2 / 87 \\
2 / N 87 \\
2 / 2 / 87 \\
2 / 2 / 87 \\
2 / 11 / 87 \\
2 / 11 / 87 \\
2 / 11 / 87 \\
2 / 11 / 87 \\
2 / 2 / 67 \\
2 / 2 / 87 \\
2 / 11 / 87 \\
2 / 11 / 87\end{array}$ & $\begin{array}{l}2 / 25 / 87 \\
2 / 25 / 87 \\
3 / \mathcal{W} 87 \\
3 / \mathbb{W} / 87 \\
W_{1 / 87} \\
V / 1 / 87 \\
5 / 13 / 67 \\
5 / 13 / 87 \\
8 / 26 / 87 \\
8 / 26 / 87\end{array}$ & $\begin{array}{l}16.22 \\
16.20 \\
16.18 \\
16.20 \\
16.19 \\
16.25 \\
16.14 \\
16.20 \\
16.19 \\
16.20 \\
16.16 \\
16.23 \\
16.21 \\
16.21\end{array}$ & $\begin{array}{l}300.62 \\
303.22 \\
295.41 \\
297.89 \\
300.95 \\
300.28 \\
298.65 \\
299.18 \\
299.59 \\
303.37 \\
297.36 \\
299.20 \\
299.23 \\
304.92\end{array}$ & $\begin{array}{l}300.64 \\
303.20 \\
295.43 \\
297.91 \\
300.97 \\
300.30 \\
290.65 \\
299.19\end{array}$ \\
\hline $\begin{array}{l}\text { BLAHR } \\
\text { EJ-13 } \\
+ \text { TUEF }\end{array}$ & $\begin{array}{l}G-559 \\
G-554 \\
G-555 \\
G-556 \\
G-557 \\
G-558 \\
G-559 \\
G-560 \\
G-561 \\
G-562 \\
G-563 \\
G-564 \\
G-565 \\
G-566\end{array}$ & $\begin{array}{l}134 \\
135 \\
135 \\
137 \\
138 \\
139 \\
140 \\
141 \\
142 \\
143 \\
144 \\
145 \\
116 \\
147\end{array}$ & $\begin{array}{l}14 \\
11 \\
28 \\
28 \\
56 \\
56 \\
91 \\
91 \\
182 \\
182 \\
260 \\
260 \\
\text { LOHG } \\
\text { LOHG }\end{array}$ & $\begin{array}{l}2 / 11 / 87 \\
2 / 11 / 87 \\
2 / 2 / 97 \\
2 / 2 / 87 \\
2 / N / 87 \\
2 / 2 / 87 \\
2 / 11 / 87 \\
2 / 11 / 87 \\
2 / 11 / 87 \\
2 / 11 / 87 \\
2 / 287 \\
2 / 2 / 87 \\
2 / 11 / 87 \\
2 / 11 / 87\end{array}$ & $\begin{array}{l}2 / 25 / 87 \\
2 / 25 / 87 \\
3 / 4 / 87 \\
3 / 4 / 87 \\
1 / 1 / 87 \\
1 / 1 / 87 \\
5 / 13 / 87 \\
5 / 13 / 87 \\
8 / 26 / 87 \\
1 / 26 / 87\end{array}$ & $\begin{array}{l}15.45 \\
15.45 \\
15.44 \\
15.49 \\
15.46 \\
15.49 \\
15.46 \\
15.54 \\
15.46 \\
15.53 \\
15.48 \\
15.54 \\
15.47 \\
15.57\end{array}$ & $\begin{array}{l}306.11 \\
300.21 \\
305.65 \\
305.02 \\
300.01 \\
299.91 \\
304.71 \\
300.40 \\
303.74 \\
298.22 \\
299.26 \\
301.01 \\
300.91 \\
301.50\end{array}$ & $\begin{array}{l}306.12 \\
300.22 \\
305.68 \\
305.85 \\
300.03 \\
299.94 \\
304.72 \\
300.42\end{array}$ \\
\hline
\end{tabular}

$\begin{array}{llll}7.71 & 24.5427 .27000 & 2.73 & 32.79 \\ 7.62 & 24.0535 .39000 & 11.34 & 33.50 \\ 7.43 & 23.7034 .64000 & 11.14 & 35.89 \\ 7.23 & 24.48 .35 .85000 & 11.37 & 42.35 \\ 7.33 & 24.5535 .71000 & 11.16 & 34.33 \\ 7.35 & 23.9535 .23000 & 11.28 & 39.43 \\ 7.50 & 24.2535 .56000 & 11.31 & 37.68 \\ 7.43 & 23.9935 .33000 & 11.34 & 34.82\end{array}$


Table A-5a (Cont'd)

\begin{tabular}{|c|c|c|c|c|c|c|c|c|c|c|c|c|}
\hline $\begin{array}{l}\text { IRST } \\
\text { TYPB }\end{array}$ & $\begin{array}{l}\text { TEST } \\
\text { MNABDR }\end{array}$ & $\begin{array}{r}\text { HZO } \\
\text { VoLumE } \\
\text { IA }\end{array}$ & $\begin{array}{l}\text { DHPLETE } \\
\text { SSEABLY } \\
\text { IH }\end{array}$ & $\begin{array}{l}\text { DOHPLETS } \\
\text { LSSFHBLY } \\
\text { OUT }\end{array}$ & MSS & $\begin{array}{l}\text { Pa } \\
\text { aur }\end{array}$ & $\begin{array}{l}\text { LPR } \\
\text { TARE }\end{array}$ & $\begin{array}{r}\text { LPE } \\
\text { VEIGTT }\end{array}$ & $\begin{array}{l}\text { SOL'h } \\
\text { VoLWWE }\end{array}$ & $\begin{array}{l}\text { IOTAL } \\
\text { CARBOH } \\
\text { PPH }\end{array}$ & $\begin{array}{r}\text { ORGAHIC } \\
\text { CAREOH } \\
\text { PPH }\end{array}$ & $\begin{array}{l}\text { SURFACE } \\
\text { ANALYSIS }\end{array}$ \\
\hline \multirow[t]{14}{*}{ ATt-1C } & $G-489$ & 14.87 & 301.59 & 301,60 & & 0.72 & 29.64 & 34.32 & 10.68 & 31.14 & 11.87 & $S E A+3 / 1 / 67$ \\
\hline & $G-484$ & 14.87 & 301.37 & 301.38 & & 6.77 & 24.63 & 35.30 & 10.67 & 31.81 & 11.83 & \\
\hline & $G-465$ & 14.91 & 308.27 & 300.27 & & 0.61 & 24.52 & 35.17 & 10.65 & 33.91 & 12.36 & $\begin{array}{r}\text { SEI 3/2̂/87 } \\
\text { SIHS }\end{array}$ \\
\hline & $G-486$ & 14.93 & 302.78 & 302.79 & & $\theta .78$ & 29.58 & 35.03 & 11.45 & 39.85 & 13.31 & \\
\hline & G-497 & 11.90 & 391.66 & 301.69 & & 9.09 & 24.23 & 34.91 & 10.69 & 36.14 & 13.69 & $\operatorname{sen} 1 / 17 / 87$ \\
\hline & G-489 & 14.90 & 308.99 & 309.00 & & 9.11 & 23.81 & 32.50 & 8.69 & 35.96 & 14.15 & \\
\hline & $G-489$ & 14.91 & 303.27 & 303.29 & & 9.50 & 22.69 & 39.30 & 10.64 & 35.03 & 15.46 & \\
\hline & $6-490$ & 14.87 & 305.05 & 305.07 & & 9.47 & 22.74 & 39.28 & 10.54 & 32.57 & 12.58 & \\
\hline & G-491 & 14.94 & 306.40 & 306.50 & & 10.07 & 23.89 & 34.60 & 10.71 & 45.45 & 19.31 & SDH $10 / 21 / 87$ \\
\hline & $G-492$ & 14.84 & 304.59 & 304.58 & & 10.08 & 24.63 & 35.15 & 10.52 & 14.84 & 19.19 & \\
\hline & G-493 & 14.91 & 303.91 & & & & & & 0.00 & & & \\
\hline & $6-494$ & 14.94 & 304.07 & & & & & & 0.00 & & & \\
\hline & $G-49$ & 14.93 & 303.35 & & & & & & 0.00 & & & \\
\hline & G-496 & 14.90 & 302.97 & & & & & & 0.00 & & & \\
\hline
\end{tabular}


Table A-5a (Cont'd)

\begin{tabular}{|c|c|c|c|c|c|c|c|c|c|c|c|}
\hline \multirow{14}{*}{$\begin{array}{l}\text { Ani-1C } \\
+ \text { TUFF }\end{array}$} & $0-497$ & 14.46 & 311.43 & 311.43 & 0.45 & 24.62 & 34.60 & 9.90 & 32.46 & 11.48 & SE: $3 / 1 / 87$ \\
\hline & G-498 & 14.47 & 335.70 & 335.71 & 6.56 & 24.52 & 34.30 & 9.86 & 40.85 & 20.80 & \\
\hline & G-499 & 14.49 & 303.68 & 303.69 & B.62 & 24.16 & 34.47 & 10.01 & 39.69 & 16,42 & $\begin{array}{r}\text { SQ1 } \\
\text { SII22/87 } \\
\text { SII7/6? }\end{array}$ \\
\hline & G-500 & 14.49 & 304.76 & 304.78 & 8.55 & 23.98 & 34.14 & 10.16 & 44.51 & 21.36 & \\
\hline & G-501 & 14.49 & 310.53 & 310.54 & 8.71 & 29.93 & 32.14 & B.21 & 41.73 & 21.59 & SEH $4 / 17 / 87$ \\
\hline & G-502 & 14.48 & 307.71 & 307.79 & 8.68 & 24.45 & 34.61 & 10.16 & 47.53 & 23.79 & \\
\hline & G-503 & 14.49 & 304.89 & 304.90 & 8.89 & 29.42 & 33.60 & 10.18 & 33.18 & 13.64 & \\
\hline & $6-504$ & 14.46 & 306.62 & 306.63 & 8.76 & 24.22 & 34.36 & 10.13 & $\$ 6.9 j$ & 20.39 & \\
\hline & G-505 & 14.53 & 338.19 & 338.19 & 9.07 & 24.55 & 34.70 & 10.15 & 57.19 & $\$ 2.91$ & \\
\hline & G-506 & 14.53 & 306.89 & 306.90 & 9.06 & 24.67 & 34.71 & 10.04 & 53.39 & 27.99 & \\
\hline & G-507 & 14.47 & 304.62 & & & & & 0.00 & & & $\operatorname{San} 10 / 21 / 67$ \\
\hline & 6-508 & 14.54 & 307.64 & & & & & 0.00 & & & \\
\hline & G-509 & 14.48 & 305.53 & & & & & 0.00 & & & \\
\hline & G-510 & {$[4.5]$} & 306.60 & & & & & 0.00 & & & \\
\hline
\end{tabular}


Table A-5a (Cont'd)

\begin{tabular}{|c|c|c|c|c|c|c|c|c|}
\hline$A \pi-6 C$ & $G-511$ & 14.87 & 309.98 & 309.99 & 8.64 & 23.64 & 35.52 & 11.88 \\
\hline & G-512 & 14.90 & 304.79 & 304.60 & 8.62 & 24.19 & 36.58 & 12.39 \\
\hline & $G-513$ & 14.07 & 302.45 & 302.46 & 8.56 & 24.60 & 36.58 & 11.95 \\
\hline & G-514 & 14.95 & 304.55 & 304.57 & 8.60 & 24.56 & 37.05 & 12.49 \\
\hline & G-515 & 14.90 & 310.77 & 310.79 & 8.98 & 23.72 & 37.57 & 11.85 \\
\hline & G-516 & 14.87 & 303.28 & 309.29 & e.92 & 24.42 & 36,43 & 12.01 \\
\hline & G-517 & 14.91 & 305.49 & 305.51 & 9.16 & 24.58 & 36.20 & 11.68 \\
\hline & G-518 & 14.91 & 306.82 & 306.83 & 9.16 & 24.45 & 36.66 & 12.21 \\
\hline & G-519 & 14.91 & 307.24 & 307.23 & 9.54 & 24.36 & 36.21 & 11.85 \\
\hline & 6-5a & 14.87 & 310.24 & 310.26 & 9.52 & 23.81 & 36.16 & 12.35 \\
\hline & G-521 & 14.94 & 302.53 & & & & & 0.00 \\
\hline & G-522 & 14.91 & 305.73 & & & & & 0.00 \\
\hline & G-523 & 14.89 & 302.65 & & & & & 0.00 \\
\hline & $6-524$ & 14.89 & 309.23 & & & & & 0.00 \\
\hline
\end{tabular}


Table A-5a (Cont'd)

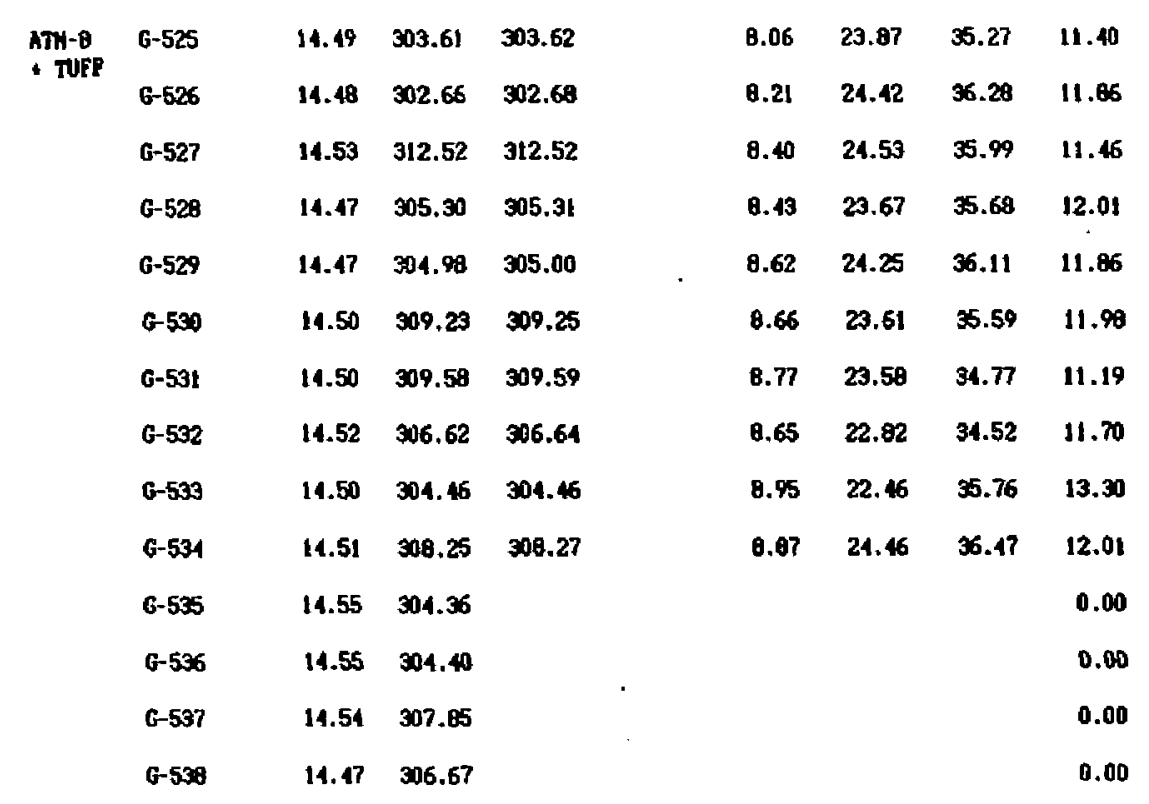


Table A-5a (Cont'd)

ORCanic

CARBOH

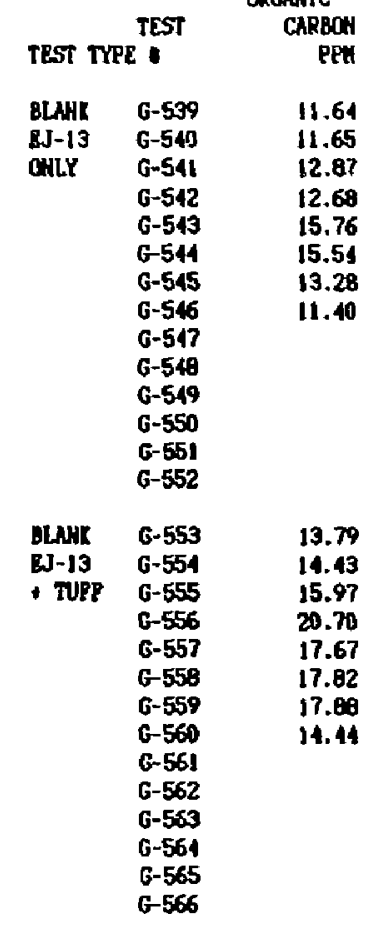


TABLE A-5b

The matrix and selected results for the OR experiments done with silicone rubber gaskets. 
Table A-Eb. Emperimental Motrix and Selected Resulta for Prelininary Experimente Done without Oanese Radiation

\begin{tabular}{|c|c|c|c|c|c|c|c|c|c|c|c|}
\hline Test Type & Tent & Sxpplo & Yeseel & $\begin{array}{l}\text { Teat } \\
\text { Length } \\
\text { (Oeyra) }\end{array}$ & Dote In & Dote Out & $\begin{array}{l}\text { Sumpl-s } \\
\text { Thi cknotet } \\
\text { (m) }\end{array}$ & & $\begin{array}{c}\text { Sanple } \\
\text { Dilanster } \\
\text { ( })\end{array}$ & $\begin{array}{c}\text { Semple } \\
\text { SA } \\
\text { Indiviguel } \\
\text { (min) }\end{array}$ & $\begin{array}{l}\text { Somple } \\
\text { Sh } \\
\text { Tot: } \\
\text { vosedf }\end{array}$ \\
\hline $\begin{array}{c}\text { Sepile } \\
\text { Unee } \\
\text { In } \\
\text { con }\end{array}$ & $\begin{array}{c}\text { Sapplo } \\
\text { unen } \\
\text { out } \\
\text { (a) }\end{array}$ & $\underset{\text { Semple }}{\text { ander }}$ & (ML) wt & $\begin{array}{c}\text { EJ-13 } \\
\text { Vol Iine } \\
\text { In } \\
(-L)\end{array}$ & $\begin{array}{c}\text { Compl ote } \\
\text { Aosiabiy } \\
\text { In } \\
\text { (c) }\end{array}$ & $\begin{array}{l}\text { Complote } \\
\text { Aosembiy } \\
\text { Out } \\
\text { (om) }\end{array}$ & Hase & Dut & $\begin{array}{l}\text { LPE } \\
\text { Ter. } \\
\text { (on) }\end{array}$ & $\underset{\text { Wight }}{\text { LPE }}$ & $\begin{array}{l}\text { Solution } \\
\text { Volume }\end{array}$ \\
\hline 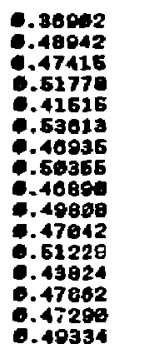 & 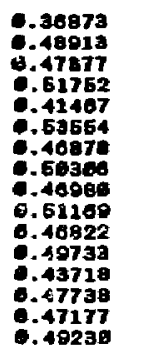 & $\begin{array}{c}29 \\
29 \\
80 \\
28 \\
48 \\
59 \\
57 \\
40 \\
\vdots \\
229 \\
160 \\
124 \\
113 \\
164\end{array}$ & $\begin{array}{l}1.6 \\
1.05 \\
1.76 \\
1.78\end{array}$ & $\begin{array}{l}14.08 \\
14.00 \\
14.00 \\
14.94 \\
14.91 \\
14.06 \\
14.95 \\
14.92\end{array}$ & $\begin{array}{l}805.25 \\
801.27 \\
201.26 \\
302.36 \\
304.20 \\
800.10 \\
305.32 \\
307.00\end{array}$ & $\begin{array}{l}306.25 \\
801.37 \\
806.28 \\
302.36 \\
304.22 \\
300.23 \\
905.24 \\
307.01\end{array}$ & $\begin{array}{l}\bullet \\
\bullet \\
. .43 \\
. . \oplus 1 \\
+. .02 \\
+. .64 \\
. .48 \\
. .68\end{array}$ & $\begin{array}{l}\bullet .76 \\
0.12 \\
. .84 \\
. .81 \\
0.02 \\
0.00 \\
0.17 \\
0.16\end{array}$ & $\begin{array}{l}23.05 \\
24.66 \\
24.47 \\
24.81 \\
24.02 \\
24.45 \\
23.01 \\
24.03\end{array}$ & $\begin{array}{l}\$ 7.50 \\
10.40 \\
90.02 \\
90.03 \\
90.12 \\
8 e .73 \\
90.91 \\
38.04\end{array}$ & $\begin{array}{l}13.06 \\
18.04 \\
14.00 \\
14.67 \\
13.92 \\
x=.20 \\
14.18 \\
14.26\end{array}$ \\
\hline
\end{tabular}

Samplen were used for purposes which excluded tiking woight lose movurcente. 
TABLE $A-6 a, b, c$

Cation Analyses ATM-1C (6a), ATM-8 (6b), and Blanks (6c), 2R

The cation results for the $2 R$ experiments are presented in this Table. The final llquid volume is the volume of liquid in the vessel at the end of the experiment. LPE weight represents the volume of solution submitted for cation analysis. ACL is the reported concentration (ppm) of the analyzed solution. ppm is the elemental concentration in the submitted leachate that has been blank corrected. Note the solutions were diluted with $20.0 \mathrm{~mL}$ of DIW prior to analysis. Blank corrections were made according to elemental trends observed in the blank results (Table $A-6 c) .(N L) ;$ is the normalized elemental mass loss for element 1 . Note the (NL) 1 values 1 isted for the experiments done using crushed glass are too large by a factor of 1.50 . 
Table A-6a. Cation Analyses, ATM-lc Glass

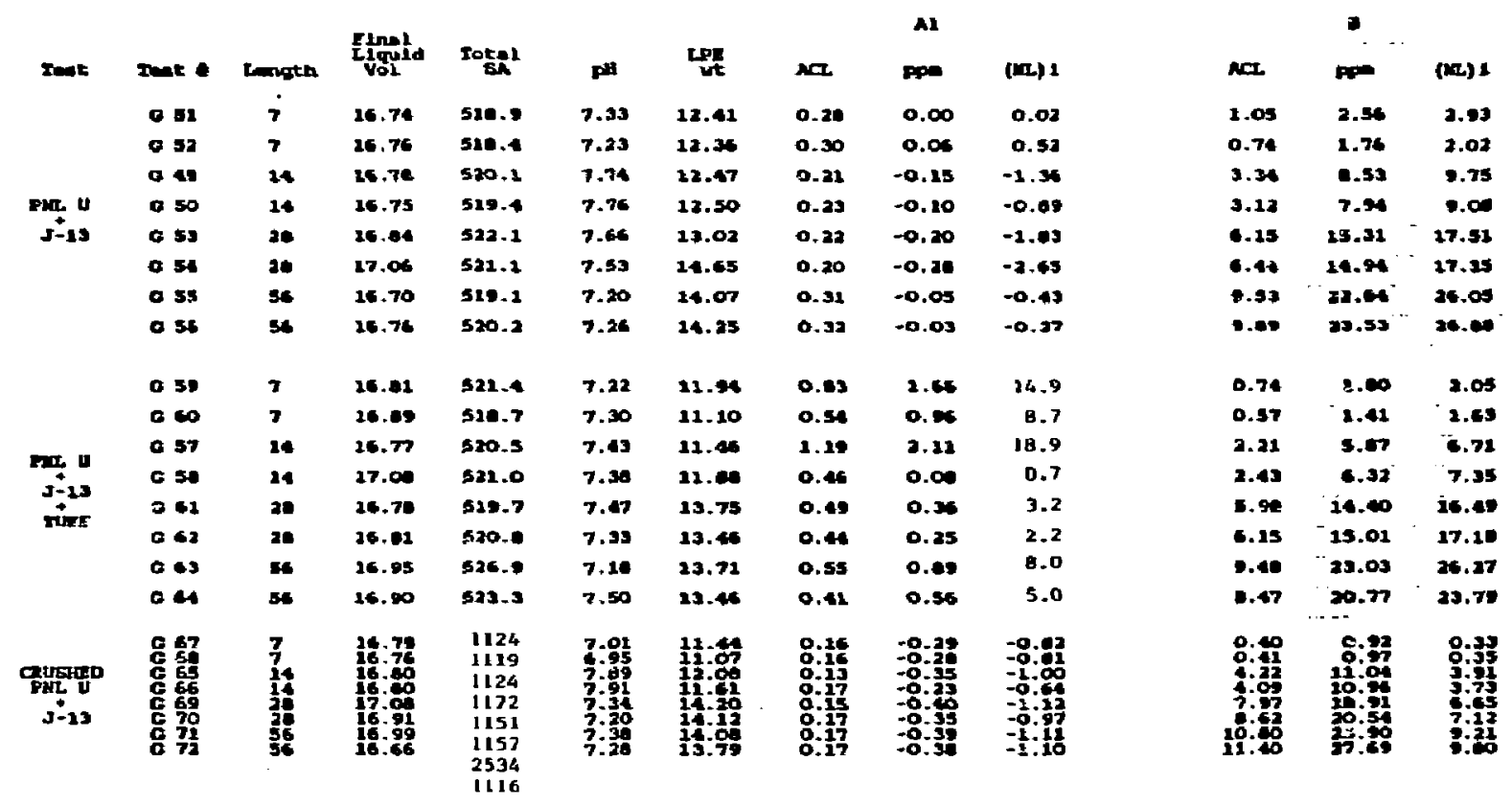


Table A-6a (Cont'd)

\begin{tabular}{|c|c|c|}
\hline Nar & EP & (nx) $)^{2}$ \\
\hline 0.066 & 0.050 & 0.61 \\
\hline 0.054 & 0.060 & 0.12 \\
\hline 0.070 & 0.100 & 0.69 \\
\hline 0.076 & 0.130 & 0.79 \\
\hline 0.640 & 1.580 & 10.35 \\
\hline 0.650 & 1.450 & 9.92 \\
\hline 0.420 & 0.870 & 6.49 \\
\hline $0.3=0$ & 0.060 & $5 . \infty 0$ \\
\hline $\begin{array}{l}0.038 \\
0.0 \times 0\end{array}$ & 0.020 & 0.14 \\
\hline 0.062 & 0.000 & 0.53 \\
\hline 0.060 & 0.000 & 0.65 \\
\hline 0.250 & 0.300 & 0.03 \\
\hline 0.110 & 0.100 & 1.24 \\
\hline 0.130 & 0.270 & 1.00 \\
\hline 0.120 & 0.220 & 1.49 \\
\hline 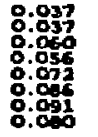 & $\begin{array}{l}0.030 \\
8: 020 \\
0: 000 \\
8: 070 \\
8: 090 \\
0: 130 \\
0.150\end{array}$ & 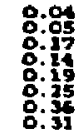 \\
\hline
\end{tabular}

\begin{tabular}{|c|c|c|c|}
\hline \multicolumn{3}{|c|}{ ca } & $C_{0}$ \\
\hline net & PFD & $(\mathbf{H I}) \mathbf{2}$ & ACI. \\
\hline 3.50 & 0.200 & 0.57 & 20.15 \\
\hline 3.44 & 0.150 & 5.30 & 0.15 \\
\hline 3.30 & 0.020 & 0.05 & co.15 \\
\hline 3.32 & 0.060 & 0.13 & 60.25 \\
\hline 3.05 & 1.360 & 2.76 & $<0.15$ \\
\hline 3.64 & 0.430 & 0.6 & $<0.15$ \\
\hline 4.10 & 0.950 & $2 . \infty$ & 60.15 \\
\hline 4.33 & 1.470 & 1.97 & $<0.15$ \\
\hline 6.13 & -2.830 & & $<0.15$ \\
\hline 5.12 & -4.900 & & 40.13 \\
\hline 5.47 & -9.010 & & 60.15 \\
\hline 6.21 & -7.370 & & $<0.15$ \\
\hline 5.72 & $-16,130$ & & $<0.13$ \\
\hline 6.95 & -13.140 & & 60.15 \\
\hline 6.10 & -20.050 & & 80.15 \\
\hline s.ee & $-25 . \infty \infty$ & & $<0.15$ \\
\hline 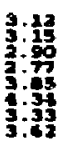 & $\begin{array}{l}-0.290 \\
-0.010 \\
-0.870 \\
-1.830 \\
\text { i.j.30 } \\
-0.900 \\
-0.0 \% 0\end{array}$ & 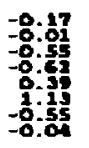 & $\begin{array}{l}20.15 \\
80.15 \\
80.15 \\
80.15 \\
80.15 \\
80.15 \\
20.15\end{array}$ \\
\hline
\end{tabular}




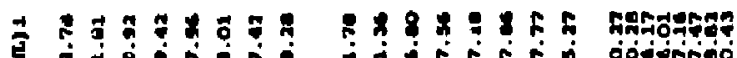

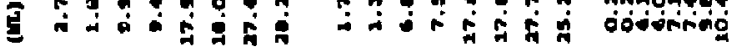

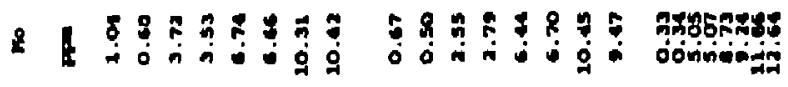

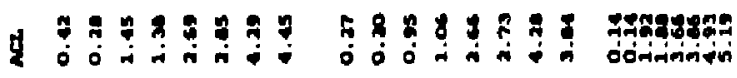

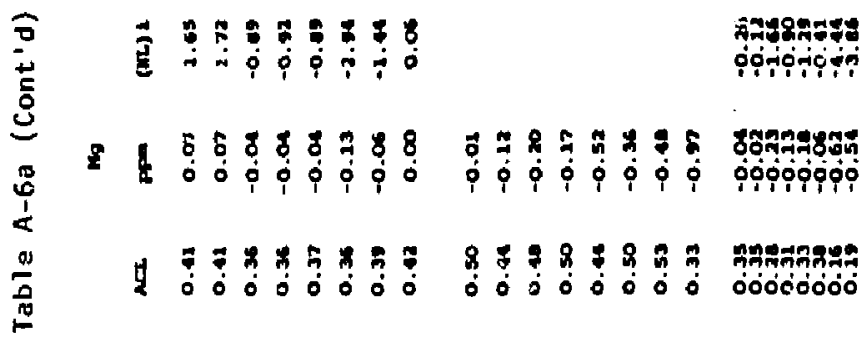

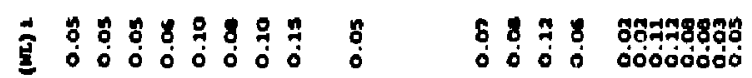

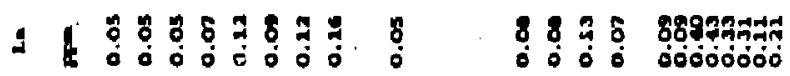
ป 
Table A-6a (Cont'd)

\begin{tabular}{|c|c|c|}
\hline & m. & \\
\hline an & PP & (th) 1 \\
\hline 11.0 & 0.63 & 3.37 \\
\hline 20.4 & 4.11 & a.14 \\
\hline 30.3 & $32 . \infty$ & 11.23 \\
\hline 29.4 & 20.54 & 10.35 \\
\hline 41.5 & $50 . \infty 0$ & 20.54 \\
\hline 4.1 & 37.65 & 20.52 \\
\hline sa.7 & 02.91 & 20.00 \\
\hline 53.4 & a.61 & 20.63 \\
\hline 20.6 & 0.21 & 2.07 \\
\hline 10.4 & 4.65 & 1.6 \\
\hline 25.5 & 23.50 & 7.60 \\
\hline 26.5 & 20.61 & - 41 \\
\hline 12.2 & 56.05 & 19.95 \\
\hline 02.2 & 39.17 & 20.41 \\
\hline 51.2 & 01.97 & 214.66 \\
\hline 4.9 & 73.43 & 25.77 \\
\hline 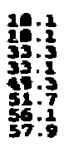 & 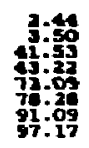 & 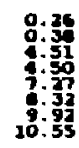 \\
\hline
\end{tabular}

\begin{tabular}{|c|c|c|c|c|c|c|}
\hline & E1 & & & & sr & \\
\hline $\mathbf{N C}$ & PPO & $(t \mathbf{L}) 1$ & & $\sin$ & $p p=$ & (an) 2 \\
\hline 20.3 & 9.40 & 1.57 & & 0.120 & 0.25 & 3.30 \\
\hline 10.1 & 3.7 & 0.63 & & 0.091 & 0.10 & 1.67 \\
\hline 32.3 & 32.64 & 3.47 & & 0.320 & 0.52 & 4.15 \\
\hline 31.3 & 25.91 & 3.01 & & 0.2230 & 0.52 & 4.84 \\
\hline$\$ .6$ & 31.09 & $=.56$ & & 0.380 & $0.7 *$ & 7.52 \\
\hline 41.0 & 45.20 & 7.67 & & 0.320 & 0.69 & 6.62 \\
\hline 47.4 & 67.06 & 21.34 & & $0.4 \times 0$ & 1.00 & 20.36 \\
\hline$\bullet .9$ & 70.63 & $11 . \bullet 1$ & & 0.470 & 1.00 & 10.07 \\
\hline 20.1 & 9.92 & 1.56 & & 0.005 & 0.23 & 2.38 \\
\hline 17.7 & 5.73 & 0.97 & & 0.046 & 0.02 & 0.40 \\
\hline 24.5 & 19.64 & 3.29 & & 0.170 & 0.36 & 3.43 \\
\hline 16.0 & 24.30 & 6.14 & & 0.150 & 0.40 & 3.0 \\
\hline 39.3 & 43.29 & 7.20 & 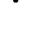 & 0.300 & 0.61 & 3.73 \\
\hline 40.4 & 47.26 & 7.92 & & 0.360 & 0.7 & 3.25 \\
\hline 47. & 63.53 & 10.41 & & 0.430 & 0.50 & $\mathbf{9 . 5 2}$ \\
\hline 93.2 & 53.39 & 9.95 & & 0.260 & 0.49 & 4.45 \\
\hline 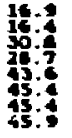 & 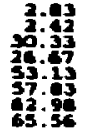 & 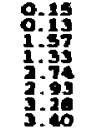 & & 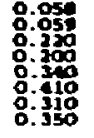 & 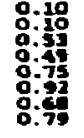 & 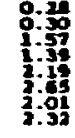 \\
\hline
\end{tabular}


Table A-6a. (Coni'd)

I1

\begin{tabular}{|c|c|c|c|c|c|}
\hline An. & Fe? & (ma) 1 & $\mathrm{ACZ}$ & FFo & $(m 2) 1$ \\
\hline 0.200 & 0.00 & 0.07 & 0.23 & 0.5s & 0.49 \\
\hline 0.010 & 0.04 & 0.07 & 0.10 & 0.42 & 0.30 \\
\hline 0.024 & 0.05 & 0.09 & 0.62 & 1.60 & 1.41 \\
\hline 0.026 & 0.05 & 0.10 & 0.55 & 1.41 & 1.27 \\
\hline 0.017 & 0.03 & 0.0 & 1.12 & 2.93 & 2.64 \\
\hline 0.020 & 0.03 & 0.06 & 0.91 & a. 30 & 2.22 \\
\hline 0.033 & 0.07 & 0.12 & 1.75 & 4.30 & 3.77 \\
\hline 0.035 & 0.07 & 0.13 & 2.13 & 3.00 & $\$ .87$ \\
\hline 0.020 & 0.04 & 0.00 & 0.14 & 0.33 & 0.30 \\
\hline 0.017 & 0.03 & 0.06 & 0.12 & 0.29 & 0.27 \\
\hline 0.019 & 0.06 & 0.07 & 0.25 & 0.63 & 0.57 \\
\hline 0.023 & 0.05 & 0.09 & 0.32 & $0 . \infty 0$ & 0.73 \\
\hline 0.019 & 0.03 & 0.06 & 0.78 & 1.90 & 1.71 \\
\hline 0.019 & 0.03 & 0.06 & 0.73 & 2.70 & 1.60 \\
\hline 0.036 & 0.07 & 0.13 & 1.36 & 3.32 & 2.80 \\
\hline 0.039 & 0.06 & 0.12 & 2.03 & 2.53 & 2.29 \\
\hline 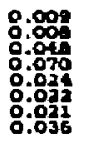 & $\begin{array}{r}-0.01 \\
-0.01 \\
0: 11 \\
0: 10 \\
0: 04 \\
0.04 \\
0.04\end{array}$ & $\begin{array}{l}\text {-0.01 } \\
\text {-0.01 } \\
\text { o:o7 } \\
0: 10 \\
0: 03 \\
0: 02 \\
0: 02 \\
0.04\end{array}$ & 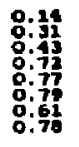 & 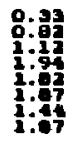 & $\begin{array}{l}0.09 \\
0: 23 \\
0: 31 \\
0: 53 \\
0: 51 \\
0: 50 \\
0: 53\end{array}$ \\
\hline
\end{tabular}

a.

\begin{tabular}{|c|c|c|}
\hline at. & PPe & $(n-C) 2$ \\
\hline 0.38 & 0.99 & 3.76 \\
\hline 0.23 & 0.65 & 2.67 \\
\hline 1.25 & 2.98 & 42.25 \\
\hline 2.02 & 3.a3 & 10.74 \\
\hline 2.90 & 3.02 & 10.05 \\
\hline $2 . \infty$ & 6.07 & 28.76 \\
\hline 2.04 & 6.00 & at.on \\
\hline 2.92 & 7.02 & 34.61 \\
\hline 0.12 & 0.51 & 2.20 \\
\hline 0.13 & 0.36 & 1.30 \\
\hline 0.58 & 2.55 & S.e9 \\
\hline 0.65 & 2.74 & 6.71 \\
\hline 2.51 & $\mathbf{9 , 7 1}$ & 14.00 \\
\hline 1.55 & 3.05 & 14.41 \\
\hline 2.24 & 5.51 & 20.76 \\
\hline $1,2 t$ & 1,75 & 17.71 \\
\hline 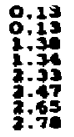 & $\begin{array}{l}0.76 \\
0.38 \\
3.85 \\
3.651 \\
5: 77\end{array}$ & $\begin{array}{l}8.42 \\
0: 43 \\
4: 31 \\
8: 15 \\
8: 55 \\
7: 55 \\
8: 50\end{array}$ \\
\hline
\end{tabular}

u

\begin{tabular}{|c|c|c|}
\hline $\mathrm{nec}$ & PF & $\left.(\mathrm{m} C)_{1}\right)$ \\
\hline 0.243 & 0.63 & 0.53 \\
\hline 0.143 & 0.37 & 0.34 \\
\hline 0.716 & 1.86 & 1.70 \\
\hline 0.657 & 1.72 & 2.56 \\
\hline 1.006 & 2.77 & 7.00 \\
\hline 3.058 & 7.26 & 6.71 \\
\hline 3.210 & 7.79 & 7.00 \\
\hline 3.110 & 7.49 & 6.02 \\
\hline $0.10 j$ & 0.45 & 0.45 \\
\hline 0.127 & 0.32 & 0.20 \\
\hline 0.634 & 1.74 & 1.50 \\
\hline 0.761 & 2.03 & 1.00 \\
\hline 1.364 & 3.34 & 3.05 \\
\hline 1.eas & 4.02 & 3.51 \\
\hline 2.054 & 5.07 & 4.56 \\
\hline 1.024 & 2.50 & 3.45 \\
\hline 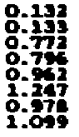 & $\begin{array}{l}0: 37 \\
0: 37 \\
2: 05 \\
3: 17 \\
3: 32 \\
3: 01 \\
2: 37 \\
2.69\end{array}$ & $\begin{array}{l}0.20 \\
8: 10 \\
0: 57 \\
8: 5 \\
8: 52 \\
8: 50 \\
0.75\end{array}$ \\
\hline
\end{tabular}


Table A-6b. Cation Analyses, ATM-8 Glass

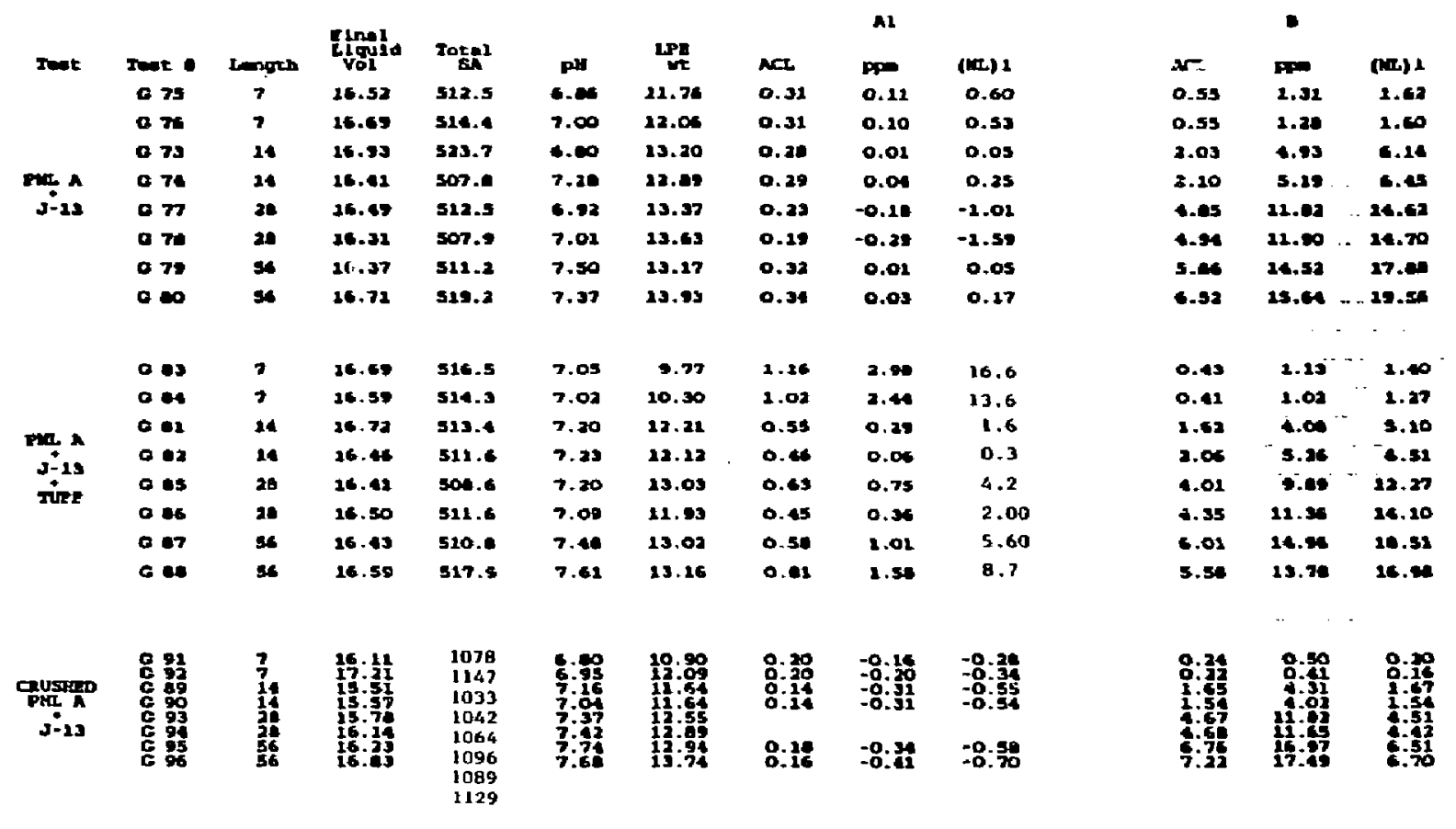


Table A-6b (Cont'd)

\begin{tabular}{|c|c|c|c|c|c|c|c|c|c|c|}
\hline \multicolumn{3}{|c|}{ a } & \multicolumn{3}{|c|}{ c. } & \multicolumn{2}{|c|}{$\infty$} & \multicolumn{3}{|c|}{ Mat } \\
\hline MI & ppon & (nt) 1 & M. & ppo & $(\mathrm{m}) 1$ & Nax & PF & & nar. & $\$$ \\
\hline 0.060 & 0.000 & 0.87 & 3.30 & 0.310 & 0.60 & $<0.15$ & & & 50.100 & \\
\hline 0.070 & 0.110 & 0.61 & 3.99 & 0.100 & 0.32 & $<0.15$ & & & $\$ 0.100$ & \\
\hline 0.120 & 0.350 & 2.01 & 4.27 & 2.170 & 4.20 & $<0.15$ & & & $<0.100$ & \\
\hline 0.100 & 0.200 & $1 . \infty 0$ & 3.96 & 2.510 & 3.00 & $<0.15$ & & & $<0.100$ & \\
\hline 0.250 & 1.060 & 5.80 & 4.77 & 3.250 & 6.30 & $<0.13$ & & & 0.110 & 0.27 \\
\hline 0.00 & 1.030 & 5.08 & A.53 & 2.520 & 4.54 & $<0.15$ & & . & 0.100 &.. \\
\hline 0.160 & 0.350 & 2.02 & 3.26 & -0.730 & -1.42 & 40.15 & & . & 0.100 & -. \\
\hline 0.150 & 0.120 & 1.42 & 3.02 & 0.360 & 0.71 & $<0.15$ & & . & $\infty .100$ & .. \\
\hline$<0.000$ & & & 5.46 & 0.250 & & 10.15 & & & $<0.100^{\circ-}$ & $\cdot$ \\
\hline 20.030 & & & 7.25 & 1.020 & & $<0.15$ & & & $c 0.100$ & $\cdots$ \\
\hline 0.060 & 0.000 & 0.83 & 7.16 & -5.140 & & 0.15 & & & $c 0.100$ & $\cdots$ \\
\hline 0.070 & 0.120 & 0.6 & 7.27 & -4.700 & & 40.15 & & & $\$ 0.200$ & 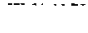 \\
\hline 0.100 & 0.370 & 2.12 & 7.65 & -10.770 & - & $<0.15$ & & & $10.100^{\circ}$ & 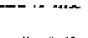 \\
\hline 0.140 & 0.290 & 2.64 & 7.96 & -8.000 & & 90.15 & & & $c 0.100$ & $\cdot \cdots$ \\
\hline 0.120 & 0.250 & 1.45 & 5.53 & -21.220 & & $<0.15$ & & & 10.100 & $-\cdots$ \\
\hline 0.097 & 0.190 & 1.10 & 4.93 & $-22-020$ & & <0.15 & & & $<0.100$ & 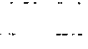 \\
\hline 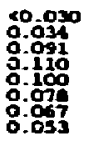 & 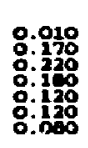 & $\begin{array}{l}0.02 \\
0: 30 \\
0: 31 \\
0: 31 \\
0: 20 \\
0: 12\end{array}$ & $\begin{array}{l}3: 10 \\
3: 25 \\
3: 20 \\
3: 37 \\
3: 51 \\
3: 57 \\
3: 94 \\
3: 16\end{array}$ & 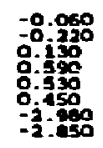 & $\begin{aligned}-0.04 \\
-0: 14 \\
0: 06 \\
0: 30 \\
0: 32 \\
0: 27 \\
-1: 71 \\
-1.73\end{aligned}$ & 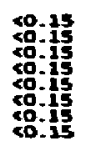 & & & $\begin{array}{l}80.100 \\
80: 100 \\
80: 100 \\
80: 100 \\
80: 100 \\
80: 100\end{array}$ & \\
\hline
\end{tabular}


Table A-6b (Cont'd)

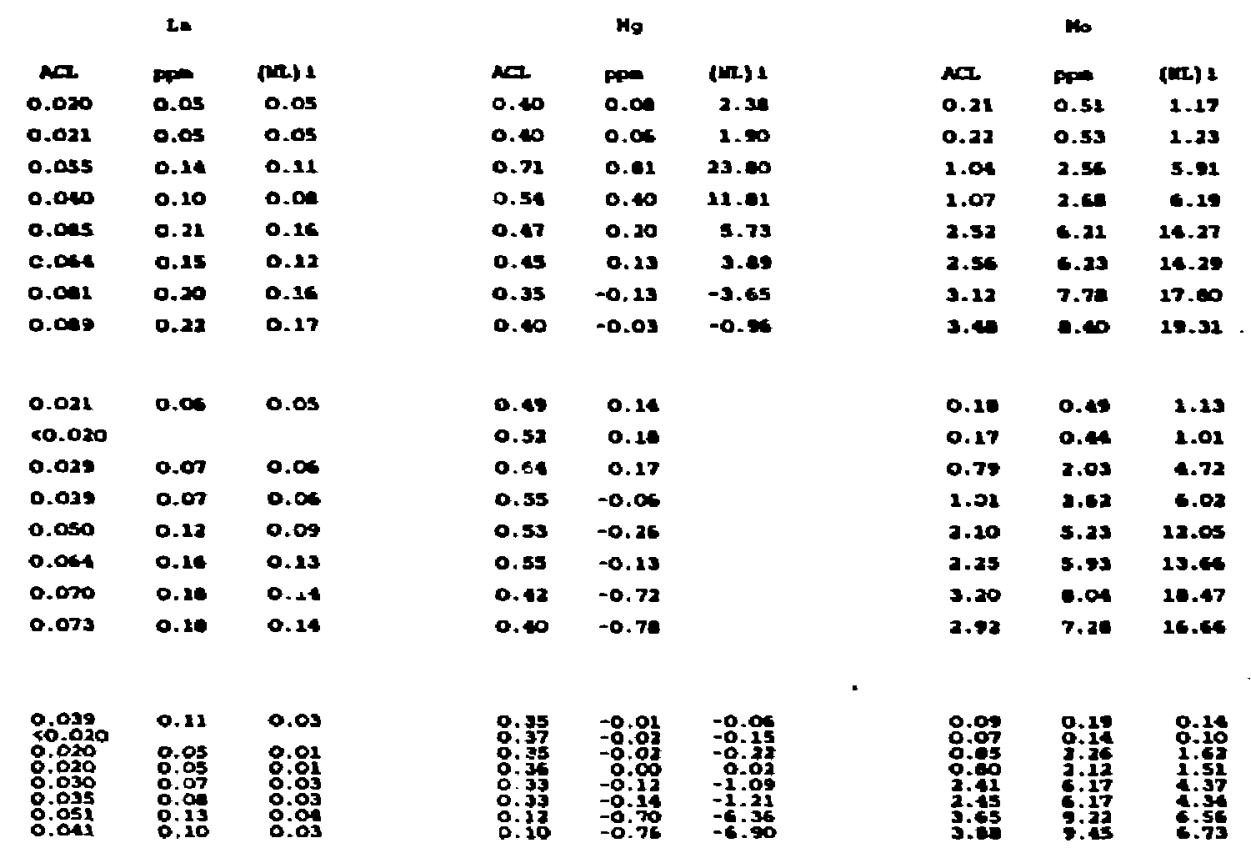


Table A-6b (Cont'd)

\begin{tabular}{|c|c|c|}
\hline ACr & Ppe & $(m) 1$ \\
\hline 20.1 & 7.25 & 2.50 \\
\hline 30.6 & 7.46 & 2.09 \\
\hline 37.3 & 21.76 & 7.51 \\
\hline 24.5 & 20.72 & 7.15 \\
\hline 39.1 & 50.50 & 17.49 \\
\hline 90.0 & 4.50 & 16.99 \\
\hline 35.3 & S4.20 & 10.55 \\
\hline 15.0 & 37.60 & 19.79 \\
\hline te.a & e.s6 & 2.96 \\
\hline 15.4 & 7.23 & 2.45 \\
\hline 24.4 & $16+47$ & s.es \\
\hline 26.0 & 21. & 7.35 \\
\hline 35.4 & 43.01 & 14.01 \\
\hline 35.1 & 47.21 & 16.25 \\
\hline 39.7 & 55.08 & 10.90 \\
\hline 30.6 & 51.62 & 22.63 \\
\hline
\end{tabular}

52

\begin{tabular}{|c|c|c|}
\hline $\begin{array}{l}\mathrm{Mat} \\
19.7\end{array}$ & 9.59 & $\begin{array}{r}\text { (MH) } 1 \\
1.44\end{array}$ \\
\hline 10.6 & 5.4.3 & $1 . \infty$ \\
\hline 27.3 & 17.20 & a.95 \\
\hline 20.0 & 19.99 & 3.43 \\
\hline 39.7 & 47.22 & e.06 \\
\hline 35.6 & 45.03 & 7.02 \\
\hline 37.6 & 47.79 & 0.12 \\
\hline 40.6 & 59.28 & 10.12 \\
\hline 19.0 & 24.04 & 2.41 \\
\hline 10.7 & 11.74 & 2.01 \\
\hline 24.9 & 26.48 & 2.05 \\
\hline 26.2 & $21 . \omega 2$ & 3.72 \\
\hline 35.4 & 36.57 & 6.26 \\
\hline 34.9 & $\$ .14$ & 0.0 \\
\hline 39.5 & $\$ 6.10$ & 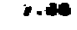 \\
\hline 38.1 & 4.52 & 7.57 \\
\hline
\end{tabular}

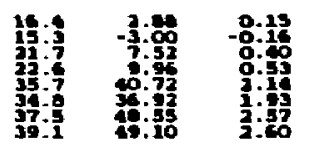

sir

\begin{tabular}{|c|c|c|}
\hline $\mathrm{NCI}$ & PFo & (ML) L \\
\hline 0.089 & 0.10 & 1.38 \\
\hline 0.007 & 0.17 & 1.33 \\
\hline 0.200 & 0.65 & 3.13 \\
\hline 0.250 & 0.59 & 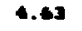 \\
\hline 0.500 & 1.20 & 0.25 \\
\hline 0.470 & 1.09 & 0.54 \\
\hline 0.033 & 0.02 & 0.12 \\
\hline 0.010 & 0.03 & 0.22 \\
\hline 0.071 & 0.12 & 0.4 \\
\hline 0.073 & 0.12 & 0.93 \\
\hline 0.290 & 0.40 & 3.25 \\
\hline 0.220 & 0.46 & 3.75 \\
\hline 0,360 & 0.79 & 4.15 \\
\hline 0.400 & 0.94 & 7.30 \\
\hline 0.340 & 0.71 & s.s. \\
\hline 0.320 & 0.42 & 4.40 \\
\hline
\end{tabular}
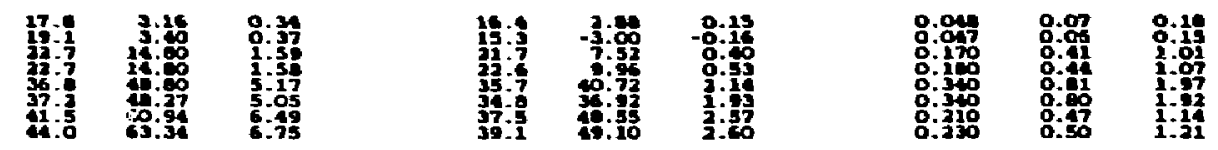
Table A-6b (Cont'd)

\begin{tabular}{|c|c|c|}
\hline $\begin{array}{c}\text { ACI } \\
0.016\end{array}$ & pes & $\begin{array}{l}\text { (kL) } \\
0.0\end{array}$ \\
\hline 0.010 & 0.03 & 0.06 \\
\hline 0.023 & 0.05 & 0.10 \\
\hline 0.026 & 0.05 & 0.0 \\
\hline 0.017 & 0.00 & 0.0 \\
\hline 0.016 & 0.09 & 0.05 \\
\hline 0.034 & n-ne & 0.1 \\
\hline 0.037 & 0.00 & 0.1 \\
\hline 0.023 & 0.0 & 0.1 \\
\hline 0.023 & 0.05 & 0.2 \\
\hline 0.023 & 0.0 & 0.0 \\
\hline 0.023 & 0.04 & 0.0 \\
\hline D.025 & 0.03 & 0.06 \\
\hline 0.017 & 0.03 & 0.0 \\
\hline 0.035 & 0.07 & 0.1 \\
\hline 0.0us & 0.00 & 0.1 \\
\hline 9.0145 & 0.03 & 0.02 \\
\hline $\begin{array}{l}0.005 \\
0.00 \% \\
0.00 \\
0.012 \\
0.013 \\
0: 013 \\
0.025\end{array}$ & $\begin{array}{l}-0.01 \\
-0: 01 \\
0: 02 \\
0: 02 \\
0.06 \\
0.05\end{array}$ & $\begin{array}{r}-0.01 \\
-0.01 \\
0.01 \\
8.01 \\
0.04 \\
0.03\end{array}$ \\
\hline
\end{tabular}

\begin{tabular}{|c|c|c|}
\hline & In & \\
\hline NCT & pes & $(k x) 2$ \\
\hline 0.18 & 0.46 & 0.42 \\
\hline 0.13 & 0.10 & D.37 \\
\hline 0.63 & 1.57 & 1.43 \\
\hline 0.50 & 2.25 & 2.20 \\
\hline 1.69 & 4.10 & 3.52 \\
\hline 1.20 & 3.27 & 2.75 \\
\hline 1.46 & 3.64 & 3.05 \\
\hline 2.60 & 3.06 & 3.25 \\
\hline 0.12 & 0.29 & 0.25 \\
\hline 0.11 & 0.20 & 0.24 \\
\hline 0.50 & 0.73 & 0.63 \\
\hline 0.35 & 0.10 & 0.51 \\
\hline 0.45 & 1.20 & 1.02 \\
\hline $0 . \infty 0$ & 2.57 & 1.32 \\
\hline $0 . \bullet 2$ & 2.05 & 1.73 \\
\hline 0.97 & 2.42 & 2.03 \\
\hline 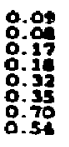 & $\begin{array}{l}0.21 \\
0: 17 \\
0: 14 \\
0: 79 \\
0.95 \\
1.74\end{array}$ & $\begin{array}{l}\text { o.os } \\
0.04 \\
0.12 \\
0.12 \\
0.21 \\
0.22 \\
0.15 \\
0.14\end{array}$ \\
\hline
\end{tabular}

c.

NaI

0.20

0.20

0.73

0.73

1.5

1.se

1.22

1.

$\begin{array}{lll}0.10 & 0.30 & 1.07\end{array}$

0.000 .240 .5

$0.41 .16 \quad 4.15$

0.21 1.03 b.0

$1.07 \quad 0.71$

$1.14 \quad 3.05$

1.33 3.37 11.0e

$1.20 \quad 3.27 \quad 21.27$

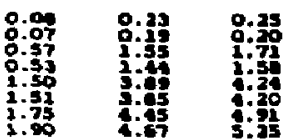

v

$\begin{array}{lll}0.1 & \text { PFe } \\ 0.125 & 0.31 & 0.38\end{array}$

$\begin{array}{lll}0.107 & 0.20 & 0.20\end{array}$

$0.67 \quad 1.71 \quad 1.56$

$0.411 .63 \quad 1.4$

2.420 .065 .51

2.3es $5.03 \quad 5.29$

1.253 $3.20 \quad 3.00$

1.230 $0.24 \quad 2.95$

$\begin{array}{lll}0.094 & 0.20 & 0.26\end{array}$

$0.020 \quad 0.27 \quad 0.25$

$0.592 \quad 1.53 \quad 1.43$

$0.710 \quad 1.00 \quad 1.73$

$1.300 \quad 3.31 \quad 3.02$

$2.008 \quad 4.51 \quad 4.21$

$2.0 \% 02.73 \quad 2.48$

1.1302 .420 .5

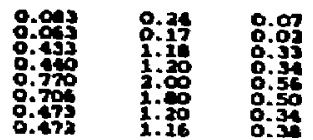


Table A-6C. Cation Analyses, Blanks

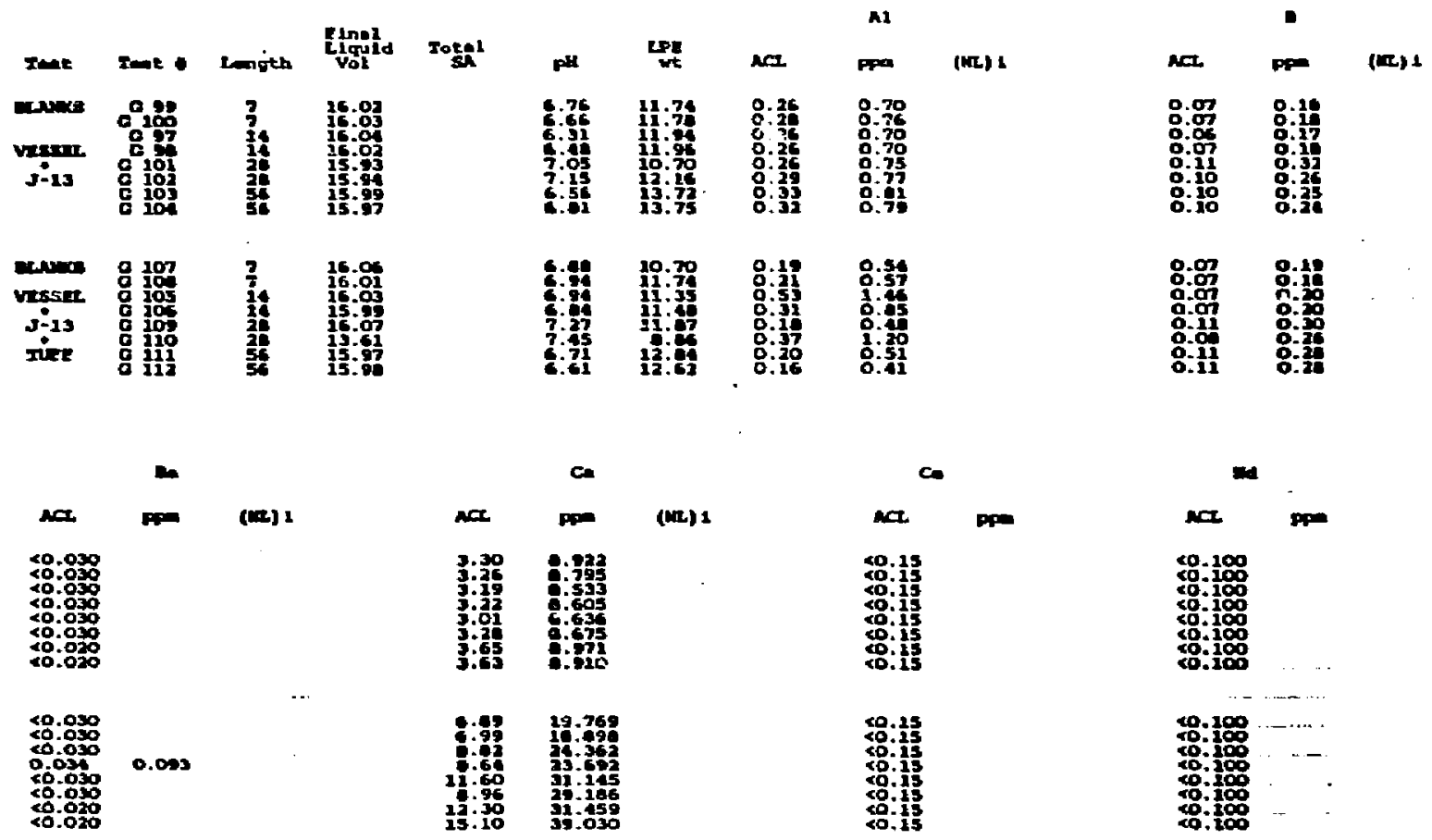


Table A-6c (Cont'd)

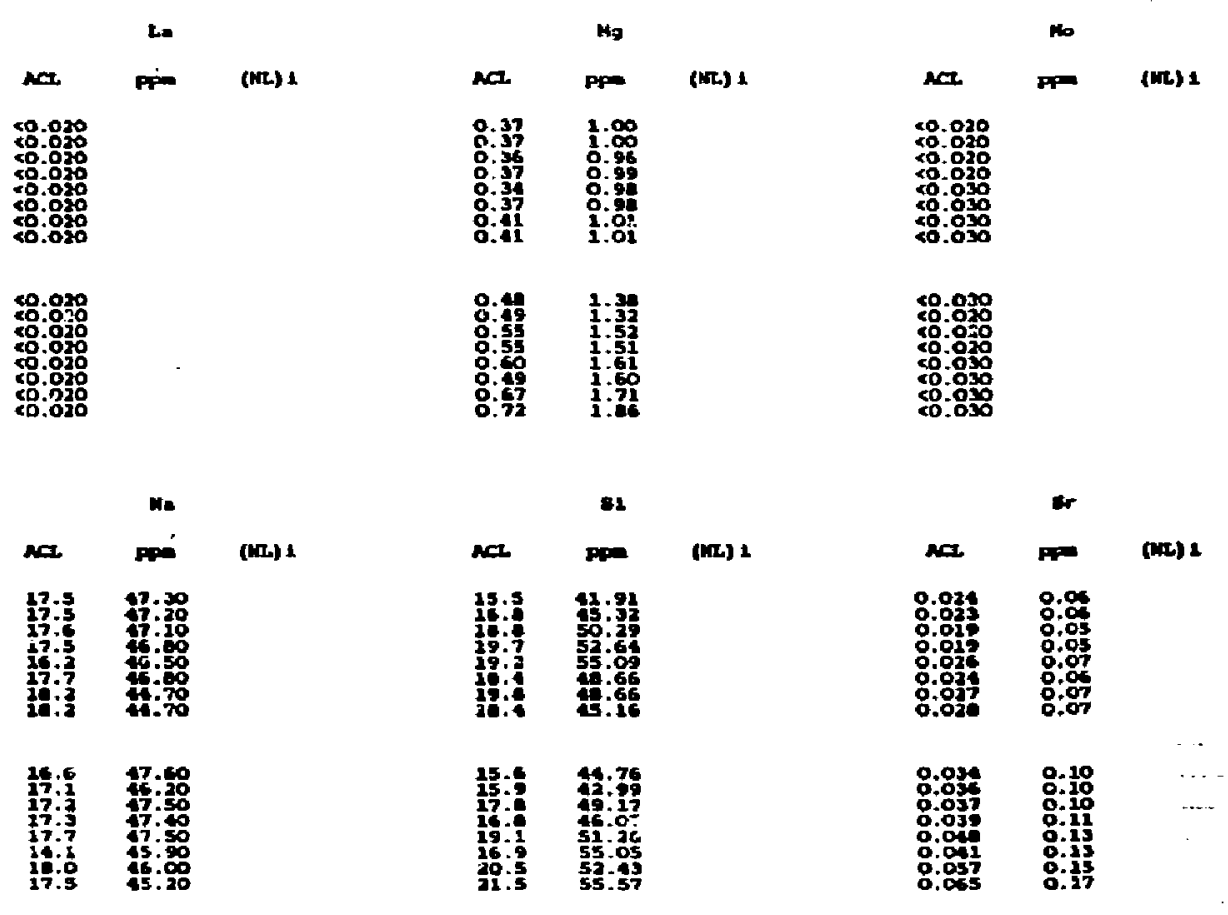


혼

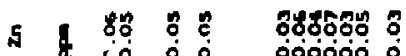

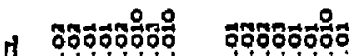

0
0
0
0
0
0
0
0
0
0

룰

- 1

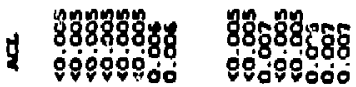

항

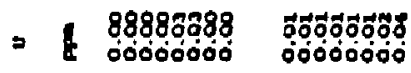

4. 88898988 898898988

$\overline{\mathrm{B}}$

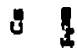

Q 
TABLE A-7

FY 1986 Gamma Cations

This Table presents the results of cation analyses for the $\mathbb{R}$ and OR (bianlss, Si gaskets) experiments. The column annotations are presented at the end of the Table. The numbers in parenthesis are the elemental weight percent concentrations for each glass type. Numbers in the Tables written as "L100" mean less than 100. 
TABLE OF COATENTS

Page

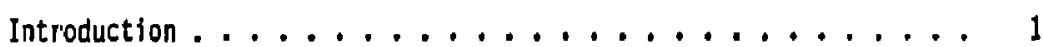

Table A-1. Composition of EJ-13 Leachants used in

Gamma Experiments ............. 3

Table A-2. Analysis of ATM-1C and ATM-8 Glasses ........ 5

Table A-3a. Experimental Matrix and Component Weight

Changes, ATM-1C Glass, 2R Experiments........ 7

Table A-3b. Experimental Matrix and Component Weight

Changes, ATH-8 GTasses ........... 10

Table A-3c. Test Matrix and Component Weight Changes,

Blanks, 2R Experiments ........... 13

Table A-4. Fy 1986 Gamma Irradiation Experiments Matrix .... 17

Table A-5a. Experimental Matrix and Selected Results for

the OR Experiments Done with Teflon Gaskets...... 24

Table A-5b, Experimental Matrix and Selected Results

for Preliminary Experiments Done Without

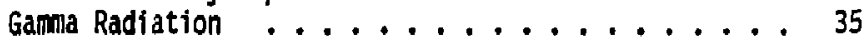

Table A-ba. Cation Analyses, ATM-lc Glass ........... 37

Table A-6b. Cation Analyses, ATM-8 Glass . . . . . . . . .. 42

Table A-6c. Catton Analyses, Blanks .............. 47

Table A-7. Cation Analyses, IR and OR (Blanks,

Sllicon Gaskets) Experiments ........... 51

Table A-8a. Cation Analyses, OR (Teflon Gasket) Experiments .... 66

Table A-8b. Solution Results for Preliminary ATM-1C

Experiments Done Without Radiation ........ 81

Table A-9. pH and Anton Analyses - Blank Samples .........83

Table A-10. Anton Results for the $I R$ and $O R$ (Stlicone Gaskets) Experiments ........... 87

Table A-11. Anion Results for the OR (Tefion) Experiments . . . . 93 


\section{TABLE OF COHTEHTS - COnt'd}

Page

Table A-12. Np and Pu Release from ATM-8 Glass . . . . . . 97

Table A-13. Np and Pu Results from the IR Experiments . . . . . 100

Table A-14. Np and Pu Results from the OR (Teflon

Gasket) Experiments ............... 105

Table A-15. SEM/EDS Analyses Performed on ATM-1C,

ATM-8 Glass . . . . . . . . . . . . 115

Table A-16. ATM-1c Glasses Profiled Using SIMS . . . . . . . 117

Fig. A-1a-f. SIMS Proftles for ATM-1c Glass . . . . . . . . . 118

Fig. A-2. SIMS Spectra of ATM-1C Glass . . . . . . . . 121

Fig. A-3. SIMS Spectra of ATM-1C Glass . . . . . . . . 123

References ..................... 125 
Table A-7. FY 1986 Gamma Cations

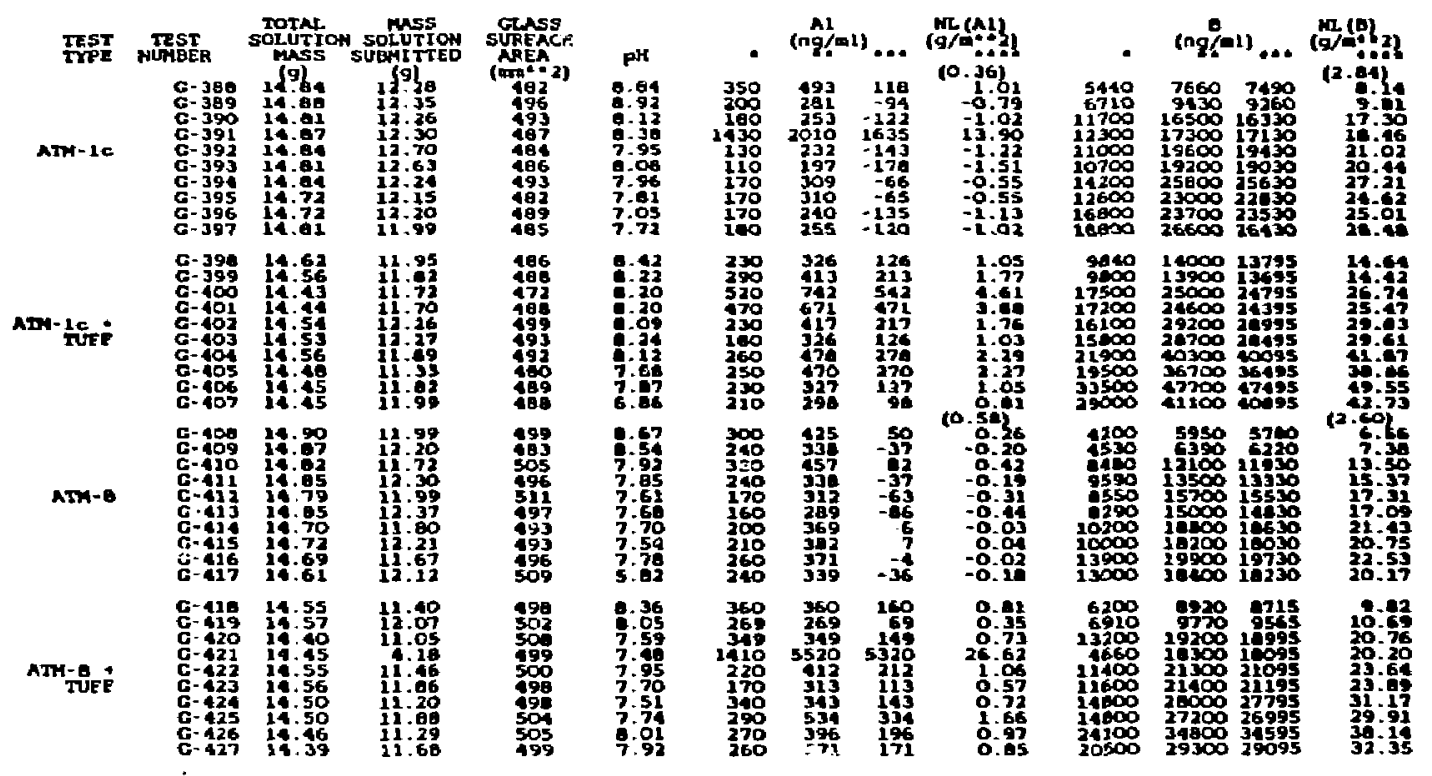


Table A-7 (Cont'd)

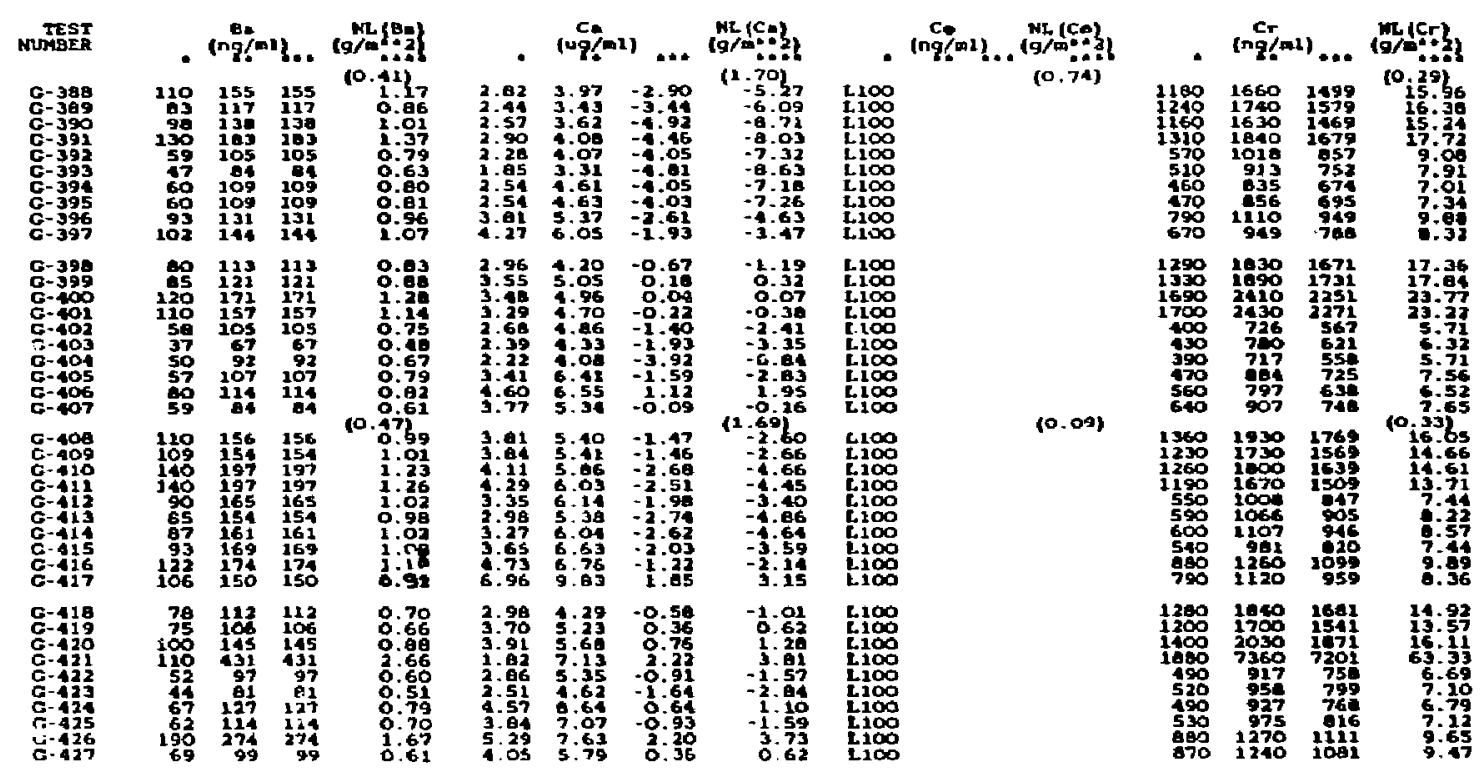


Table A-7 (Cont'd)

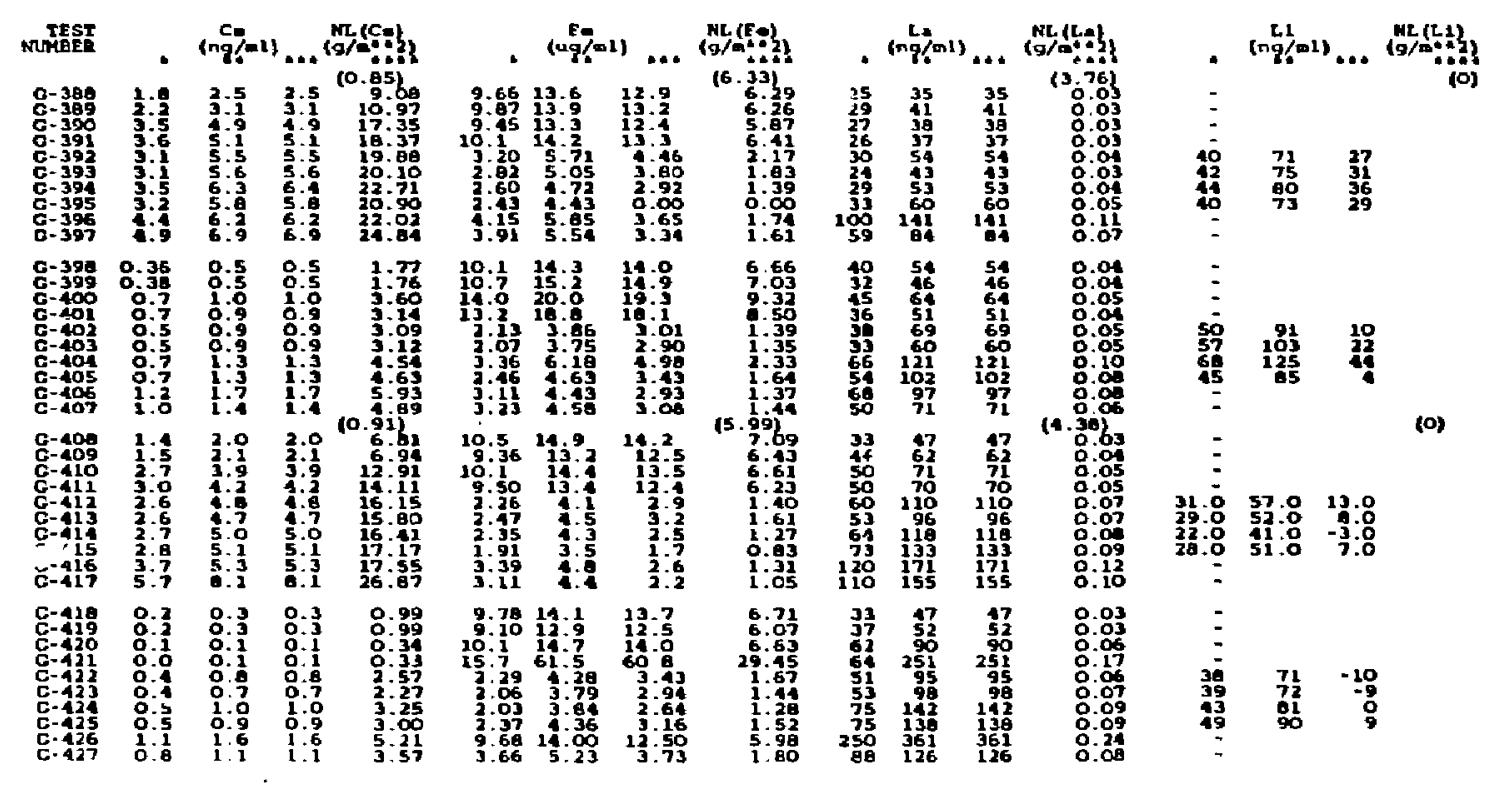


Table A-7 (Cont'd)

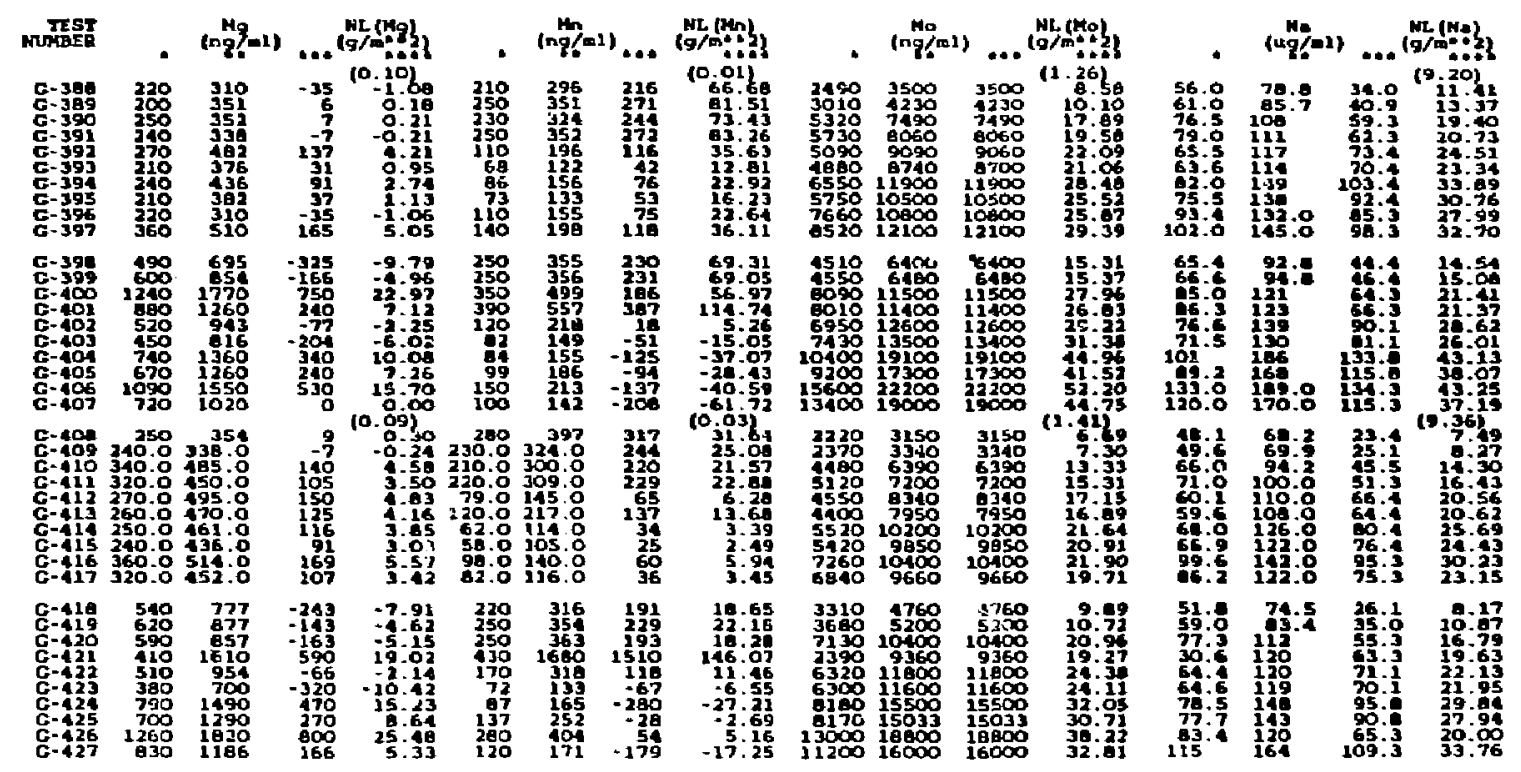


Table A-7 (Cont'd)

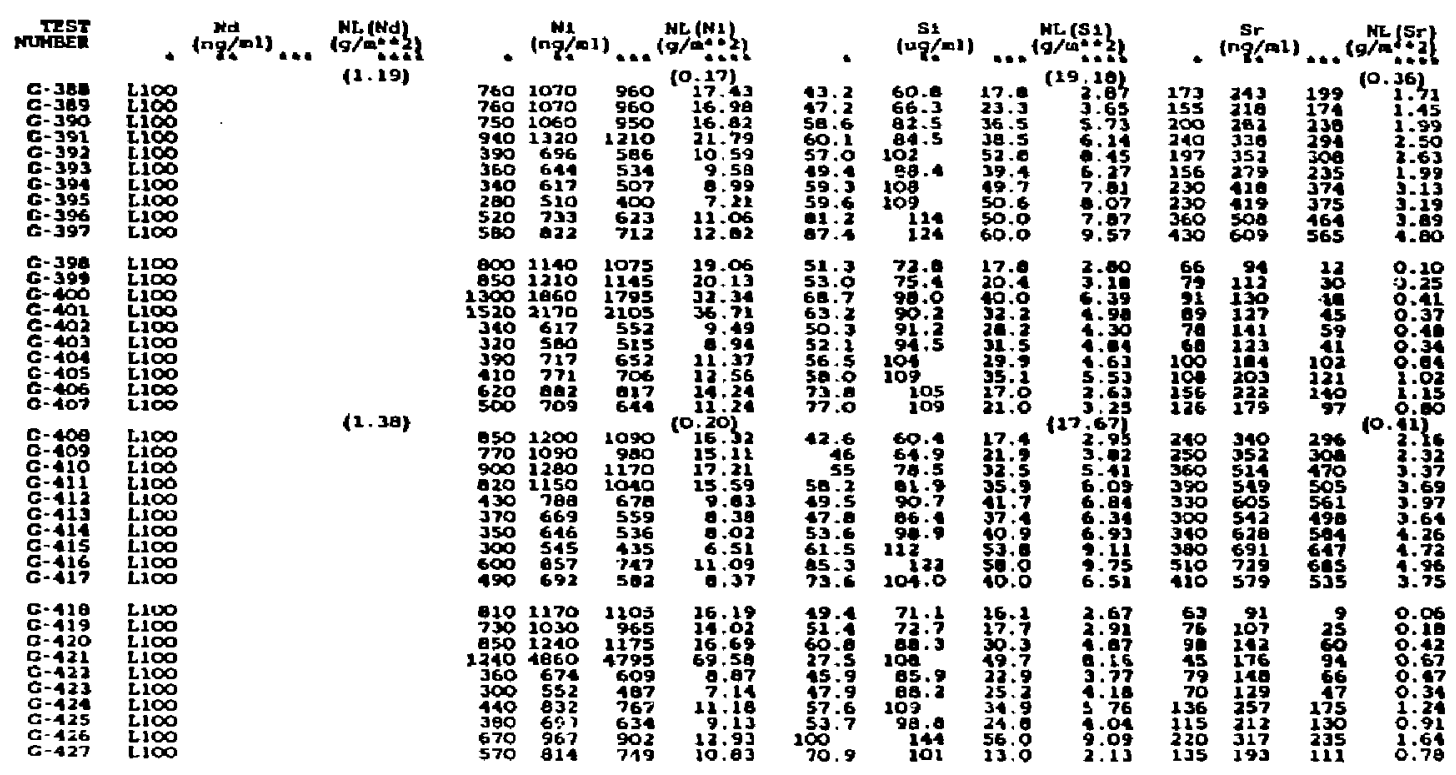


Table A-7 (Cont'd)

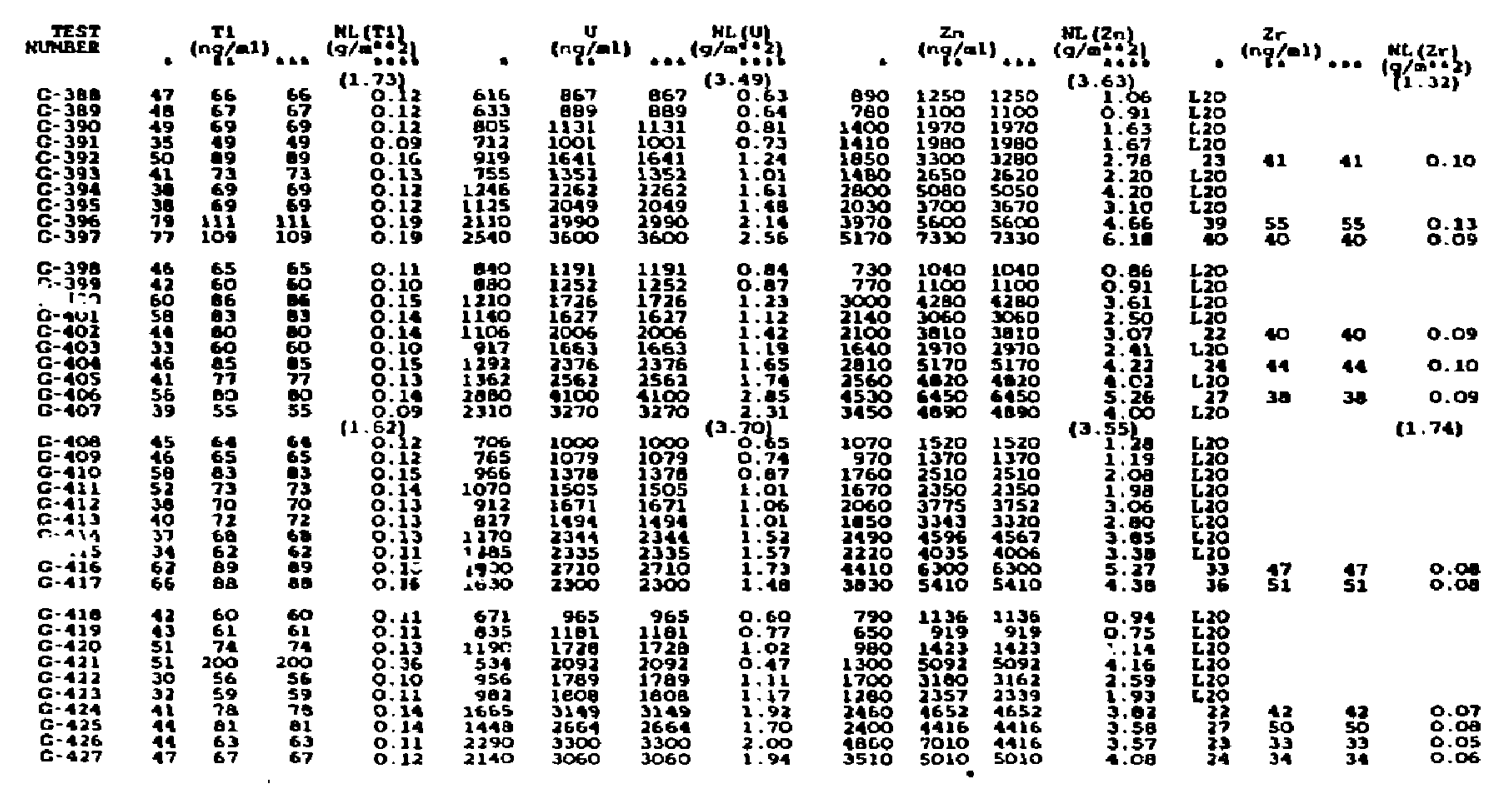


Table A-7 (Cont'd)

\begin{tabular}{|c|c|c|c|c|c|c|c|c|c|c|}
\hline \multirow{2}{*}{ TEST } & \multirow{2}{*}{ 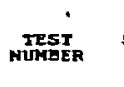 } & \multirow{2}{*}{\multicolumn{3}{|c|}{$\begin{array}{l}\text { TOTAL } \\
\text { soLUTIO soluss } \\
\text { (g) } \\
\text { (g) }\end{array}$}} & \multicolumn{3}{|c|}{$\underset{(n \mathbf{g} / \mathbf{A} 1)}{\mathbf{A}}$} & \multicolumn{3}{|c|}{ (ngial) } \\
\hline & & & & & $\mathrm{PH}$ & $\cdot$ & $\cdots$ & - & $\cdots$ & \\
\hline $25-13$ & 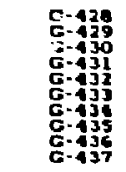 & $\begin{array}{l}15: 41 \\
15: 43 \\
16: 11 \\
16: 11 \\
16: 11 \\
16: 12 \\
16: 05 \\
15: 95 \\
15: 97\end{array}$ & $\begin{array}{r}13.49 \\
13.91 \\
13.82 \\
13.83 \\
14.93 \\
14.69 \\
13.79 \\
13.63 \\
13.72\end{array}$ & $\begin{array}{r}5 \\
5 \\
5 \\
5 \\
10 \\
10 \\
10 \\
10 \\
5 \\
5\end{array}$ & $\begin{array}{l}7.14 \\
7.39 \\
6.57 \\
6.75 \\
6.36 \\
5.54 \\
6.51 \\
6.79 \\
6.91\end{array}$ & $\begin{array}{l}300 \\
370 \\
290 \\
240 \\
200 \\
190 \\
310 \\
370 \\
390 \\
300\end{array}$ & $\begin{array}{l}111 \\
513 \\
3131 \\
327 \text { (375) } \\
347 \text { (375) } \\
325 \text { (375) } \\
363 \text { (375) } \\
360 \text { (375) } \\
396 \text { (375) }\end{array}$ & $\begin{array}{l}120 \\
160 \\
115 \\
121 \\
51 \\
110 \\
106 \\
100 \\
101\end{array}$ & $\begin{array}{l}164 \\
222 \\
157 \\
165 \\
87 \\
185 \\
197 \\
149 \\
547\end{array}$ & $\begin{array}{l}(170) \\
(170) \\
(170) \\
(170) \\
(170)\end{array}$ \\
\hline EJ-13:- & 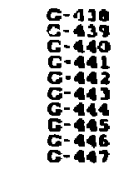 & $\begin{array}{l}15: 49 \\
15: 37 \\
15: 47 \\
15: 43 \\
15: 35 \\
15: 37 \\
15: 32 \\
15: 35 \\
15: 32\end{array}$ & 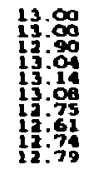 & $\begin{array}{r}5 \\
5 \\
5 \\
5 \\
10 \\
10 \\
10 \\
10 \\
5 \\
5\end{array}$ & $\begin{array}{l}7.03 \\
2.05 \\
6.41 \\
6: 30 \\
6.70 \\
6.71 \\
6.04 \\
6.82 \\
6.80\end{array}$ & 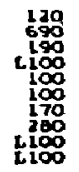 & $\begin{array}{r}166 \\
355(200) \\
264 \\
176(200) \\
176(200) \\
203 \\
302(200) \\
(200)\end{array}$ & $\begin{array}{l}150 \\
190 \\
160 \\
150 \\
97 \\
115 \\
110 \\
115 \\
139 \\
139\end{array}$ & $\begin{array}{l}200 \\
263 \\
222 \\
206 \\
171 \\
167 \\
196 \\
206 \\
193\end{array}$ & $\begin{array}{l}(205) \\
(205) \\
(205) \\
(205) \\
(205)\end{array}$ \\
\hline No cosion & 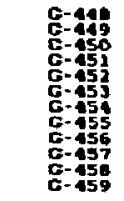 & $\begin{array}{l}16.19 \\
16.15 \\
15.42 \\
15.14 \\
16.16 \\
16.27 \\
16.28 \\
16.10 \\
15.05 \\
15.97 \\
15.65 \\
15.80\end{array}$ & $\begin{array}{l}14.16 \\
14: 19 \\
13: 20 \\
13: 07 \\
13: 79 \\
13.72 \\
13.80 \\
13.58 \\
13.70 \\
13.56 \\
13: 37 \\
13.55\end{array}$ & $\begin{array}{r}5 \\
5 \\
5 \\
5 \\
5 \\
5 \\
10 \\
10 \\
10 \\
10 \\
5 \\
5\end{array}$ & 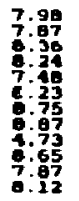 & $\begin{array}{l}300 \\
370 \\
330 \\
330 \\
240 \\
230 \\
190 \\
170 \\
190 \\
2100 \\
380 \\
350\end{array}$ & $\begin{array}{l}106 \\
133 \\
1360) \\
145(360) \\
327 \\
317(360) \\
3140 \\
310 \\
394(360) \\
311 \\
365(360) \\
345 \\
342(360)\end{array}$ & $\begin{array}{r}130 \\
130 \\
190 \\
130 \\
110 \\
114 \\
80 \\
86 \\
85 \\
86 \\
116 \\
96\end{array}$ & $\begin{array}{l}176 \\
175 \\
262 \\
150 \\
150 \\
156 \\
130 \\
119 \\
117 \\
149 \\
136\end{array}$ & $\begin{array}{l}\text { (150) } \\
\text { (150) } \\
\text { (150) } \\
\text { (150) } \\
\text { (150) } \\
\text { (150) }\end{array}$ \\
\hline 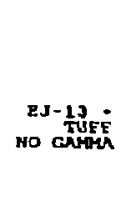 & 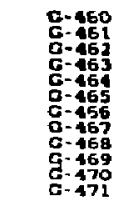 & $\begin{array}{l}15.45 \\
15.44 \\
15.44 \\
15.44 \\
15.41 \\
15.37 \\
15.47 \\
15.43 \\
1538 \\
15.29 \\
15.39\end{array}$ & $\begin{array}{l}13.20 \\
13.16 \\
13.01 \\
12.87 \\
12.88 \\
12.86 \\
12.69 \\
12.84 \\
12.93 \\
12.83 \\
12.65 \\
12.86\end{array}$ & $\begin{array}{r}5 \\
5 \\
5 \\
5 \\
5 \\
10 \\
10 \\
10 \\
10 \\
5\end{array}$ & $\begin{array}{l}7.27 \\
7: 38 \\
9: 06 \\
7: 90 \\
7: 62 \\
8: 35 \\
8: 27 \\
7: 98 \\
8.12 \\
6.04 \\
0.02\end{array}$ & $\begin{array}{r}130 \\
130 \\
100 \\
140 \\
100 \\
1100 \\
160 \\
140 \\
260 \\
190 \\
4100 \\
100\end{array}$ & $\begin{array}{r}179(210) \\
179 \\
139(210) \\
199 \\
296(210) \\
296(210) \\
161 \\
331(210) \\
(210)\end{array}$ & $\begin{array}{l}190 \\
160 \\
1150 \\
150 \\
140 \\
140 \\
123 \\
170 \\
124 \\
118 \\
117 \\
112\end{array}$ & $\begin{array}{l}282 \\
221 \\
235 \\
309 \\
194 \\
194 \\
230 \\
302 \\
220 \\
192 \\
163 \\
197\end{array}$ & $\begin{array}{l}(205) \\
(205) \\
(205) \\
(205) \\
(205) \\
(205)\end{array}$ \\
\hline
\end{tabular}


Table A-7 (Cont'd)

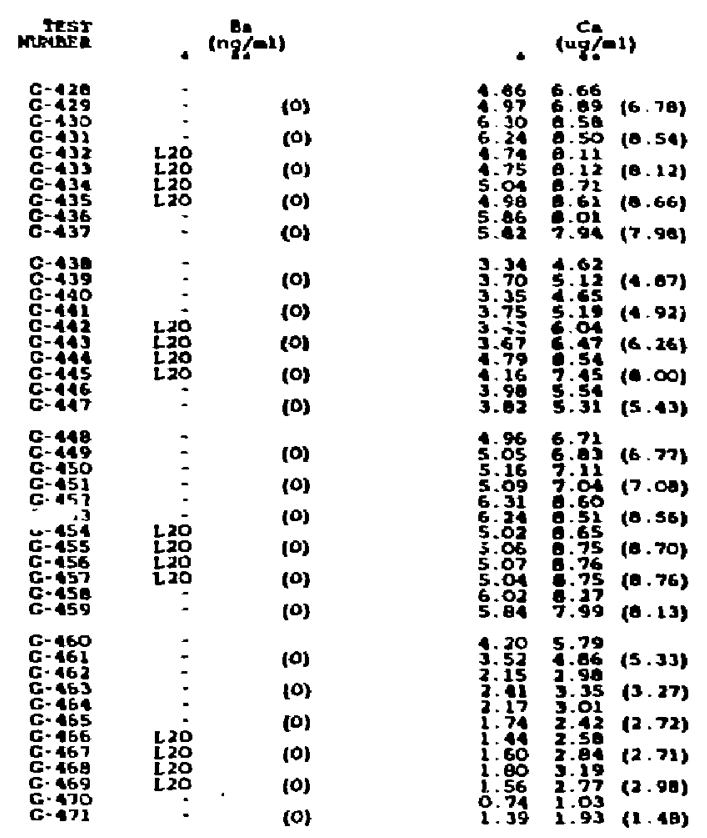

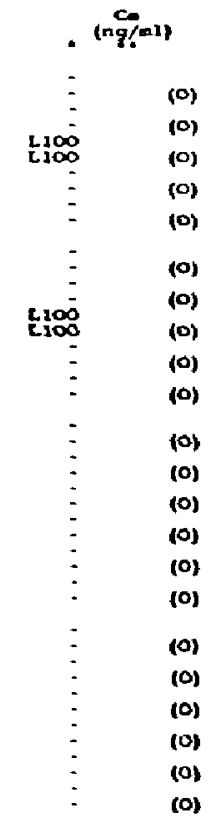

- (ng/ $r$-1)

(o)

(o)

161
$=\quad 16(16)$

(o)

(o)

(o)

(o) 


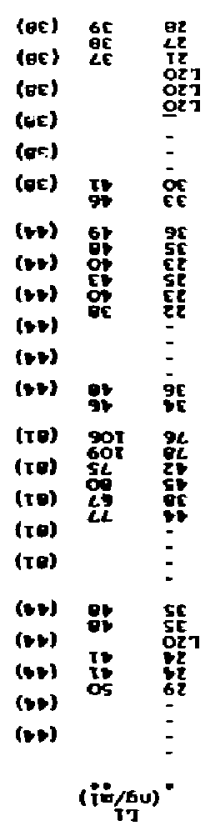

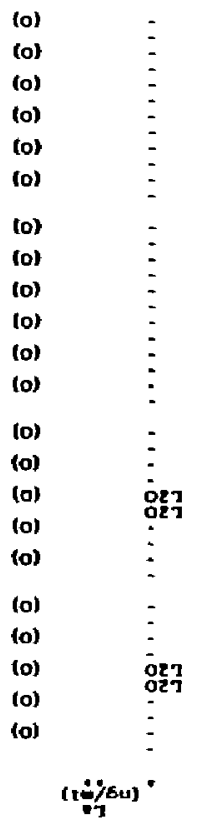

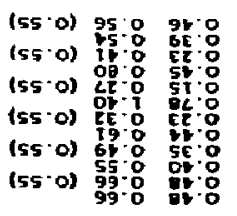

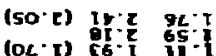

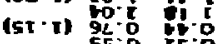

(06.0) 5.:

$\left(05^{\circ} 0\right)$ 25.

(x-o) 56:1

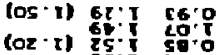

(so-o) IS:

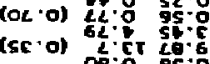

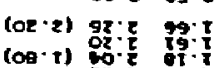

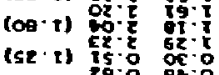

(s6 a) $29: 0$ :日:

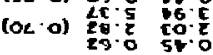

$\left(r \rightarrow h_{n}\right)$

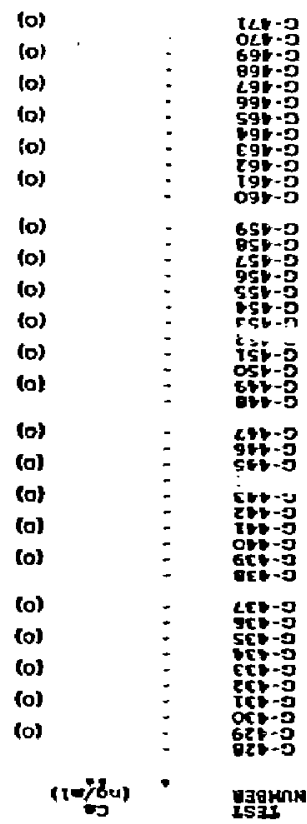

$(p, q u 03)<-\forall$ alqe 
Table A-7 (Cont'd)
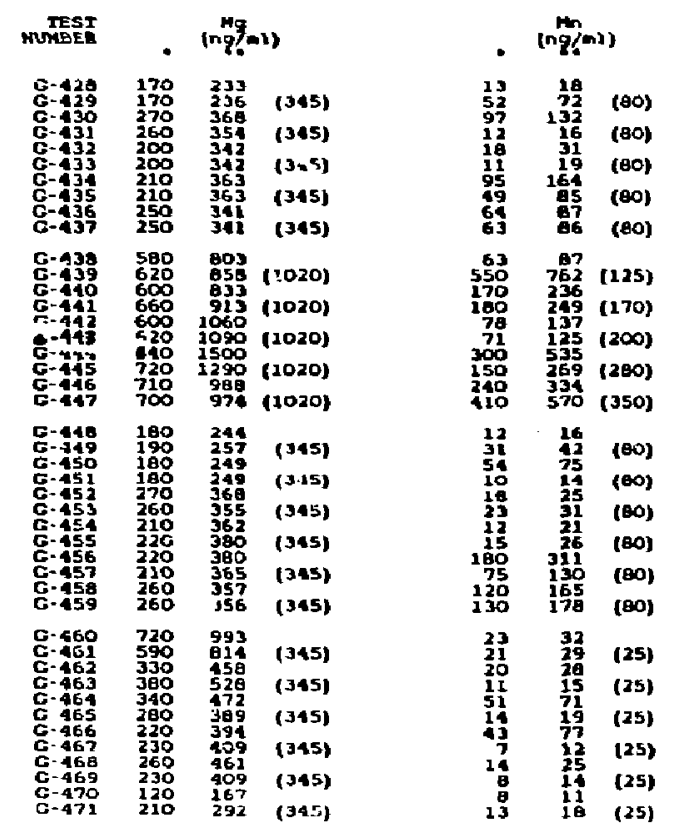

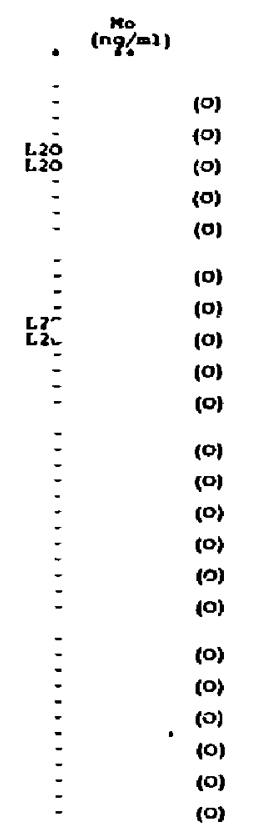

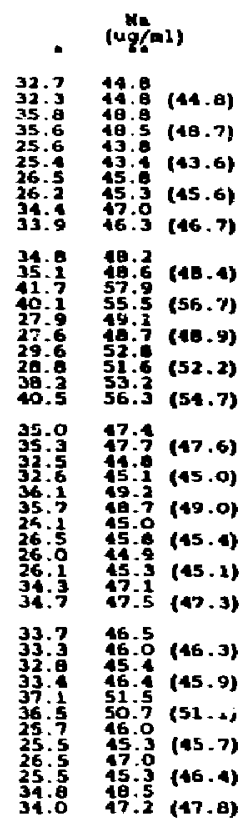


Table A-7 (Cont'.d)

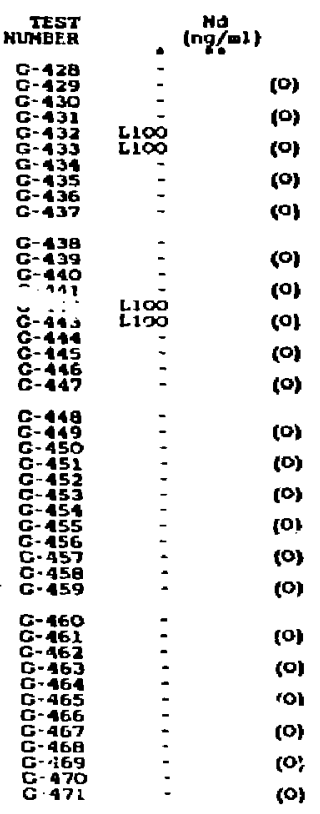
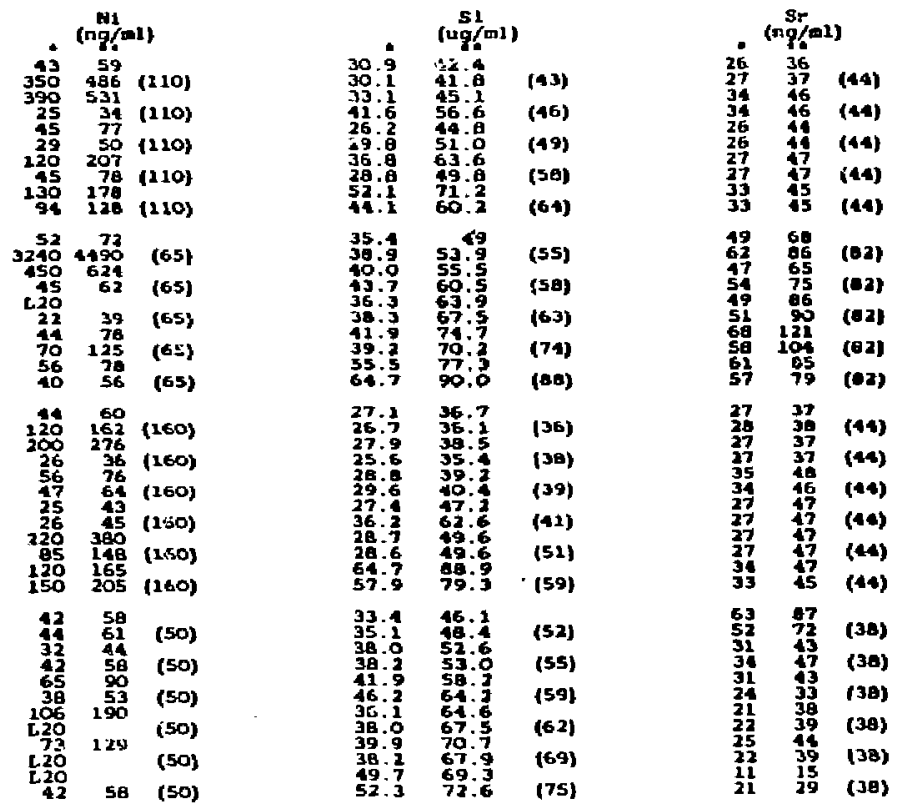


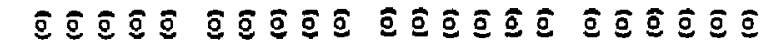
通

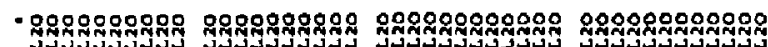

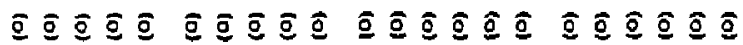

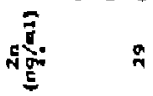

ت

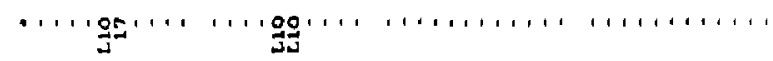

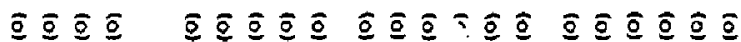

点

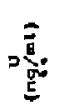

g

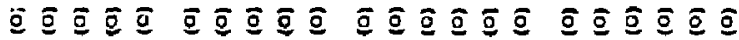

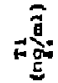

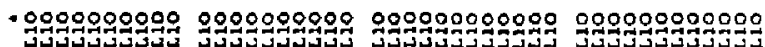

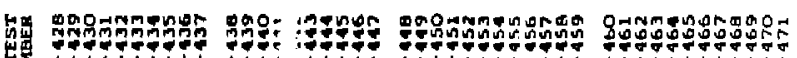

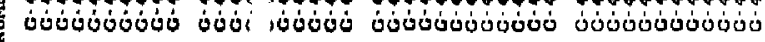




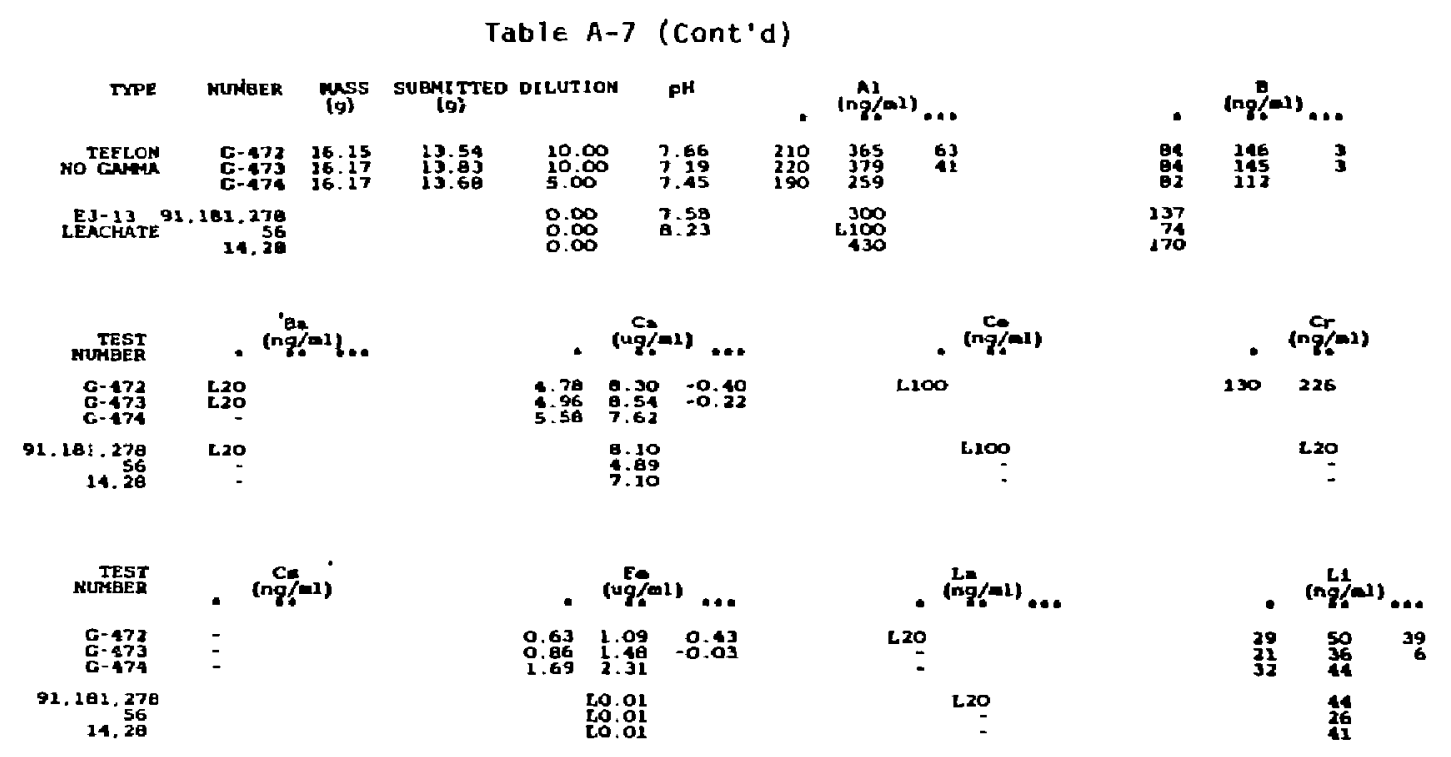

:.. ACL RESULT

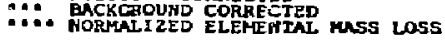


Table A-7 (Cont'd)

\begin{tabular}{|c|c|c|c|}
\hline MLMAST & . & (ng ${ }^{n} q_{(1)}$ & \\
\hline 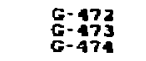 & $\begin{array}{l}210 \\
3100 \\
250\end{array}$ & $\begin{array}{l}\begin{array}{c}265 \\
362 \\
342\end{array} \\
342\end{array}$ & -16 \\
\hline $\begin{array}{r}11.181 .278 \\
14.56\end{array}$ & & $\begin{array}{l}340 \\
2100 \\
250\end{array}$ & \\
\hline Muthest & & $\operatorname{lng}^{n \dot{d}}$ & \\
\hline $\begin{array}{l}\mathbf{c}-42 \\
\mathbf{g}-473 \\
6-474\end{array}$ & 1.100: & & \\
\hline $\begin{array}{r}91.181 .278 \\
14,26\end{array}$ & & 1100 & \\
\hline $\begin{array}{l}\text { TEST } \\
\text { MUMGEE }\end{array}$ & . & 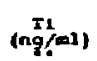 & \\
\hline $\begin{array}{l}6-473 \\
6=473 \\
6-474\end{array}$ & $\begin{array}{l}410 \\
410 \\
t 10\end{array}$ & & \\
\hline $\begin{array}{c}91.181 .278 \\
14.28\end{array}$ & & $\begin{array}{l}21 \\
11 \\
21\end{array}$ & \\
\hline
\end{tabular}

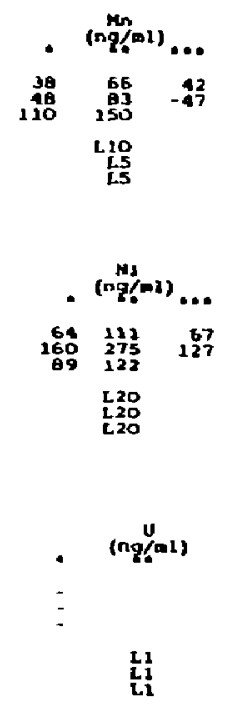
- $\operatorname{(nq}_{a+1}^{m o}$
Lio
L20

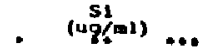
$\begin{array}{lll}31 & 37 \\ 21 & 37: 2 & -17.7 \\ 23: 3 & 36.7 & -12: 9\end{array}$
34:5
- $\left.\operatorname{lng}^{2 r} / 21\right)$
1319
- 4ro

- (uqRa1) $\ldots$

$\begin{array}{lll}25.5 & 14.5 & -0.9 \\ 254: 5 & 13: 9 & -1: 3\end{array}$

14:4

. (ngir 2 (n)

$\begin{array}{lll}26 & 45 & -2 \\ 32 & 16 & -1\end{array}$

$\frac{14}{24}$

181.278
14.26

. (ng? (n) $^{-1)}$

$\mathrm{L} 20$
$\mathrm{~L} 20$
$\mathrm{E} 20$

$\mathrm{L} 20$
$\mathrm{~L} 20$
$\mathrm{~L} 20$ 


\section{TABLE A-8a}

Cation analyses from the OR (Tefion gasket) experiments are presented in this Table. The columns are as follows:

- raw data reported by the Analytical Chemistry Laboratory (ACL)

** concentration corrected for $5 \mathrm{~mL}$ dllution with DIW

*** background corrected concentration

$\star \star \star \star$ normalized mass loss 
Table A-8a. Cation Analyses from the OR (Teflon Gasket) Experiments

\begin{tabular}{|c|c|c|c|c|c|c|c|c|c|c|c|c|}
\hline \multirow{2}{*}{$\begin{array}{l}\text { TEST } \\
\text { TYPE }\end{array}$} & \multirow{2}{*}{$\begin{array}{l}\text { TEST } \\
\text { MUABER }\end{array}$} & \multirow{2}{*}{$\begin{array}{r}\text { SAMPLE } \\
\text { SA } \\
\text { TOTAL' } \\
\text { VESSEL }\end{array}$} & \multirow{2}{*}{$\begin{array}{r}\text { H20 } \\
\text { voLdisE } \\
\text { JH }\end{array}$} & \multirow{2}{*}{$\begin{array}{l}\text { SOL'H } \\
\text { YOLOHE }\end{array}$} & \multicolumn{2}{|c|}{$\begin{array}{r}A l \\
\text { (ng/al) }\end{array}$} & \multicolumn{2}{|c|}{$\begin{array}{l}\text { ML(A) } \\
(g / m 2)\end{array}$} & \multicolumn{2}{|c|}{$\begin{array}{r}B \\
(n g / 01)\end{array}$} & \multicolumn{2}{|c|}{$\begin{array}{l}\mathrm{HC}(B) \\
(g / D 2)\end{array}$} \\
\hline & & & & & ! & "1 & 10: & Hen: & - & "1 & nat & $n+1$ \\
\hline ATH-IC & $\begin{array}{l}G-483 \\
G-484 \\
G-485 \\
G-486 \\
G-487 \\
G-488 \\
G-489 \\
G-490 \\
G-491 \\
G-492 \\
G-493 \\
G-494 \\
G-495 \\
G-496\end{array}$ & $\begin{array}{l}463 \\
460 \\
457 \\
476 \\
466 \\
465 \\
455 \\
464 \\
457 \\
464 \\
463 \\
461 \\
460 \\
458\end{array}$ & $\begin{array}{l}14.87 \\
14.87 \\
14.91 \\
14.93 \\
14.90 \\
14.90 \\
14.91 \\
14.87 \\
14.94 \\
14.94 \\
14.91 \\
14.94 \\
14.93 \\
14.90\end{array}$ & $\begin{array}{r}10.68 \\
10.67 \\
10.65 \\
11.45 \\
10.68 \\
0.69 \\
10.64 \\
10.54 \\
10.71 \\
10.52\end{array}$ & $\begin{array}{l}440 \\
130 \\
330 \\
270 \\
240 \\
260 \\
210 \\
200 \\
260 \\
230\end{array}$ & $\begin{array}{l}646 \\
631 \\
485 \\
388 \\
352 \\
410 \\
309 \\
295 \\
381 \\
339\end{array}$ & $\begin{array}{r}-79 \\
-94 \\
-240 \\
-337 \\
-373 \\
-315 \\
-361 \\
-375 \\
-325 \\
-367\end{array}$ & $\begin{array}{r}-.7 \\
-.6 \\
-2.2 \\
-2.9 \\
-3.2 \\
-2.8 \\
-3.3 \\
-3.3 \\
-2.9 \\
-3.3\end{array}$ & $\begin{array}{r}550 \\
620 \\
1420 \\
1580 \\
5190 \\
5200 \\
8980 \\
8200 \\
13900 \\
15000\end{array}$ & $\begin{array}{r}807 \\
911 \\
2087 \\
2270 \\
7620 \\
8192 \\
13200 \\
12090 \\
20243 \\
22129\end{array}$ & $\begin{array}{r}650 \\
762 \\
1938 \\
2121 \\
7471 \\
6043 \\
13051 \\
11941 \\
20094 \\
21980\end{array}$ & $\begin{array}{r}.7 \\
.9 \\
2.2 \\
2.3 \\
8.1 \\
9.1 \\
15.1 \\
13.5 \\
29.1 \\
24.8\end{array}$ \\
\hline $\begin{array}{l}\text { ATH-IC } \\
\text { TUFF }\end{array}$ & $\begin{array}{l}G-497 \\
G-498 \\
G-499 \\
G-500 \\
G-501 \\
G-502 \\
6-503 \\
6-504 \\
G-505 \\
G-506 \\
G-507 \\
G-508 \\
6-509 \\
G-510\end{array}$ & $\begin{array}{l}469 \\
467 \\
459 \\
479 \\
475 \\
472 \\
463 \\
457 \\
463 \\
459 \\
475 \\
466 \\
460 \\
472\end{array}$ & $\begin{array}{l}14.46 \\
14.47 \\
14.49 \\
14.49 \\
34.49 \\
14.48 \\
14.49 \\
14.46 \\
14.53 \\
14.53 \\
14.47 \\
14.54 \\
14.48 \\
14.51\end{array}$ & $\begin{array}{r}9.98 \\
9.86 \\
10.01 \\
10.16 \\
8.21 \\
10.16 \\
10.18 \\
10.13 \\
10.15 \\
10.04\end{array}$ & $\begin{array}{l}180 \\
210 \\
240 \\
170 \\
250 \\
170 \\
170 \\
200 \\
250 \\
290\end{array}$ & $\begin{array}{l}270 \\
316 \\
360 \\
254 \\
370 \\
254 \\
253 \\
299 \\
373 \\
434\end{array}$ & $\begin{array}{r}392 \\
367 \\
221 \\
124 \\
88 \\
146 \\
144 \\
130 \\
237 \\
195\end{array}$ & $\begin{array}{l}3.3 \\
3.2 \\
1.9 \\
1.0 \\
.7 \\
1.2 \\
1.2 \\
1.1 \\
2.1 \\
1.7\end{array}$ & $\begin{array}{l}1800 \\
2960 \\
3950 \\
4370 \\
5900 \\
6180 \\
6400 \\
7320 \\
7260 \\
7770\end{array}$ & $\begin{array}{c}2702 \\
1461 \\
5923 \\
6521 \\
9443 \\
9221 \\
9543 \\
10933 \\
10836 \\
11640\end{array}$ & $\begin{array}{r}2525 \\
4284 \\
5746 \\
6344 \\
9316 \\
9044 \\
9366 \\
10756 \\
10659 \\
11463\end{array}$ & $\begin{array}{r}2.7 \\
4.7 \\
6.4 \\
6.8 \\
10.0 \\
9.8 \\
10.3 \\
12.6 \\
11.8 \\
12.8\end{array}$ \\
\hline
\end{tabular}


Table A-8a (Cont'd)

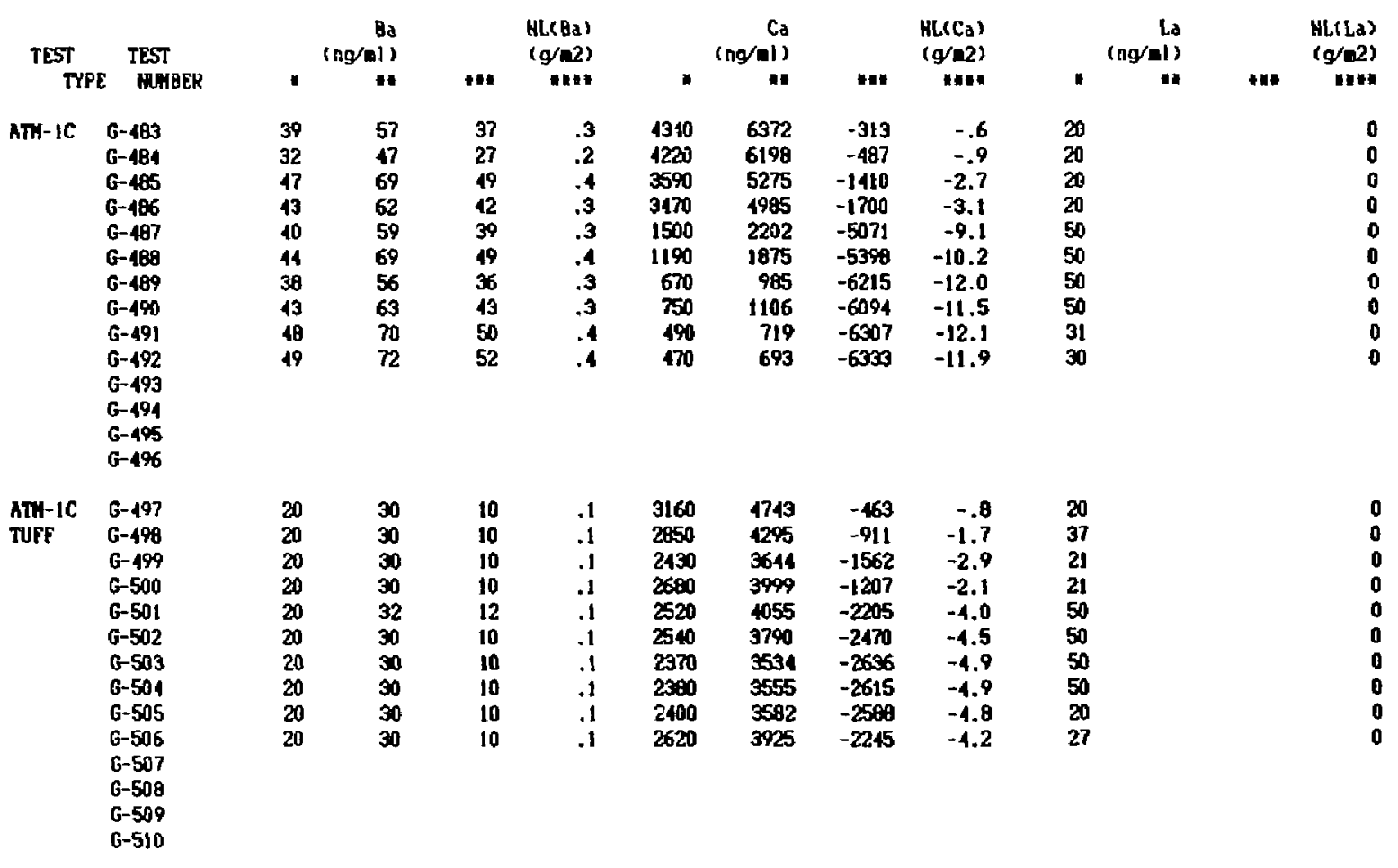


Table A-8a (Cont'd)

\begin{tabular}{|c|c|c|c|c|c|c|c|c|c|c|c|c|c|}
\hline TEST & TEST & & $\begin{array}{l}\mathrm{Hg} \\
(\mathrm{el})\end{array}$ & & $\begin{array}{l}\text { u(Hg) } \\
9^{\prime}(-2)\end{array}$ & & $\begin{array}{r}\text { Ho } \\
y / 01)\end{array}$ & & (4) & & $\begin{array}{r}\text { Ha } \\
g / a \mid)\end{array}$ & & $\begin{array}{l}\text { HL(Ha) } \\
\left(g\left(g^{\prime}\right)\right.\end{array}$ \\
\hline TYP & E MNBER & - & 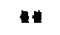 & un & nen & 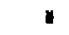 & 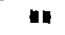 & $w$ & mat & 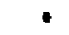 & * & Wh & une. \\
\hline ATH-IC & $\begin{array}{l}G-483 \\
G-484 \\
G-485 \\
G-486 \\
G-487 \\
G-489 \\
G-489 \\
G-490 \\
G-491 \\
G-492 \\
G-499 \\
G-494 \\
G-495 \\
G-496\end{array}$ & $\begin{array}{l}130 \\
110 \\
140 \\
130 \\
220 \\
210 \\
220 \\
200 \\
330 \\
300\end{array}$ & $\begin{array}{l}191 \\
162 \\
206 \\
187 \\
323 \\
331 \\
323 \\
295 \\
484 \\
443\end{array}$ & $\begin{array}{r}35 \\
6 \\
50 \\
31 \\
167 \\
175 \\
153 \\
125 \\
329 \\
287\end{array}$ & $\begin{array}{r}.7 \\
.1 \\
1.0 \\
.6 \\
3.2 \\
3.5 \\
3.1 \\
2.5 \\
6.7 \\
5.7\end{array}$ & $\begin{array}{r}210 \\
200 \\
580 \\
640 \\
2240 \\
2300 \\
3900 \\
3600 \\
6170 \\
6730\end{array}$ & $\begin{array}{r}308 \\
294 \\
652 \\
919 \\
3269 \\
3623 \\
5950 \\
5308 \\
9050 \\
9929\end{array}$ & $\begin{array}{r}258 \\
214 \\
602 \\
869 \\
3239 \\
3573 \\
5600 \\
5258 \\
9000 \\
9879\end{array}$ & $\begin{array}{r}.7 \\
.6 \\
2.1 \\
2.2 \\
7.9 \\
9.1 \\
15.1 \\
13.4 \\
23.4 \\
25.1\end{array}$ & $\begin{array}{l}37.8 \\
38.0 \\
41.5 \\
42.0 \\
55.3 \\
53.1 \\
66.5 \\
63.3 \\
83.0 \\
89.1\end{array}$ & $\begin{array}{r}55.5 \\
55.8 \\
61.0 \\
60.3 \\
81.2 \\
83.7 \\
97.8 \\
93.3 \\
122.9 \\
131.9\end{array}$ & $\begin{array}{r}5.2 \\
5.5 \\
10.7 \\
10.0 \\
30.9 \\
33.4 \\
47.5 \\
43.0 \\
72.6 \\
81.6\end{array}$ & $\begin{array}{r}1.8 \\
1.9 \\
3.8 \\
3.4 \\
10.3 \\
11.6 \\
16.9 \\
15.0 \\
25.6 \\
28.4\end{array}$ \\
\hline $\begin{array}{l}\text { MTH-IC } \\
\text { JUFt }\end{array}$ & $\begin{array}{l}G-497 \\
G-498 \\
G-499 \\
G-500 \\
G-501 \\
G-502 \\
G-503 \\
G-504 \\
G-505 \\
G-506 \\
G-507 \\
G-50 B \\
G-509 \\
G-510\end{array}$ & $\begin{array}{r}130 \\
110 \\
110 \\
120 \\
90 \\
120 \\
200 \\
110 \\
230 \\
330\end{array}$ & $\begin{array}{l}195 \\
166 \\
165 \\
179 \\
145 \\
179 \\
298 \\
164 \\
343 \\
494\end{array}$ & $\begin{array}{r}-79 \\
-108 \\
-109 \\
-95 \\
-129 \\
-95 \\
24 \\
-110 \\
69 \\
220\end{array}$ & $\begin{array}{r}-1.5 \\
-2.1 \\
-2.2 \\
-1.8 \\
-2.5 \\
-1.8 \\
.5 \\
-2.2 \\
1.4 \\
4.4\end{array}$ & $\begin{array}{l}720 \\
1300 \\
1760 \\
1900 \\
2650 \\
2770 \\
2950 \\
3280 \\
3190 \\
3440\end{array}$ & $\begin{array}{l}1081 \\
1959 \\
2639 \\
2954 \\
4254 \\
4133 \\
4250 \\
4999 \\
1761 \\
5153\end{array}$ & $\begin{array}{l}1031 \\
1909 \\
2509 \\
2904 \\
4214 \\
4083 \\
4200 \\
4649 \\
1711 \\
5103\end{array}$ & $\begin{array}{r}2.5 \\
4.7 \\
6.5 \\
7.0 \\
10.2 \\
9.9 \\
10.4 \\
12.2 \\
11.7 \\
12.8\end{array}$ & $\begin{array}{l}39.4 \\
41.6 \\
14.9 \\
45.0 \\
46.3 \\
50.1 \\
66.5 \\
69.3 \\
50.9 \\
51.5\end{array}$ & $\begin{array}{l}59.1 \\
62.7 \\
67.3 \\
67.1 \\
74.5 \\
74.8 \\
99.2 \\
94.5 \\
75.8 \\
77.1\end{array}$ & $\begin{array}{r}8.2 \\
11.0 \\
16.4 \\
16.2 \\
23.6 \\
23.9 \\
49.9 \\
45.2 \\
26.3 \\
27.6\end{array}$ & $\begin{array}{r}2.8 \\
4.0 \\
5.6 \\
5.3 \\
7.8 \\
8.0 \\
17.0 \\
15.6 \\
9.0 \\
9.5\end{array}$ \\
\hline
\end{tabular}


Table A-Ba (Cont'd)

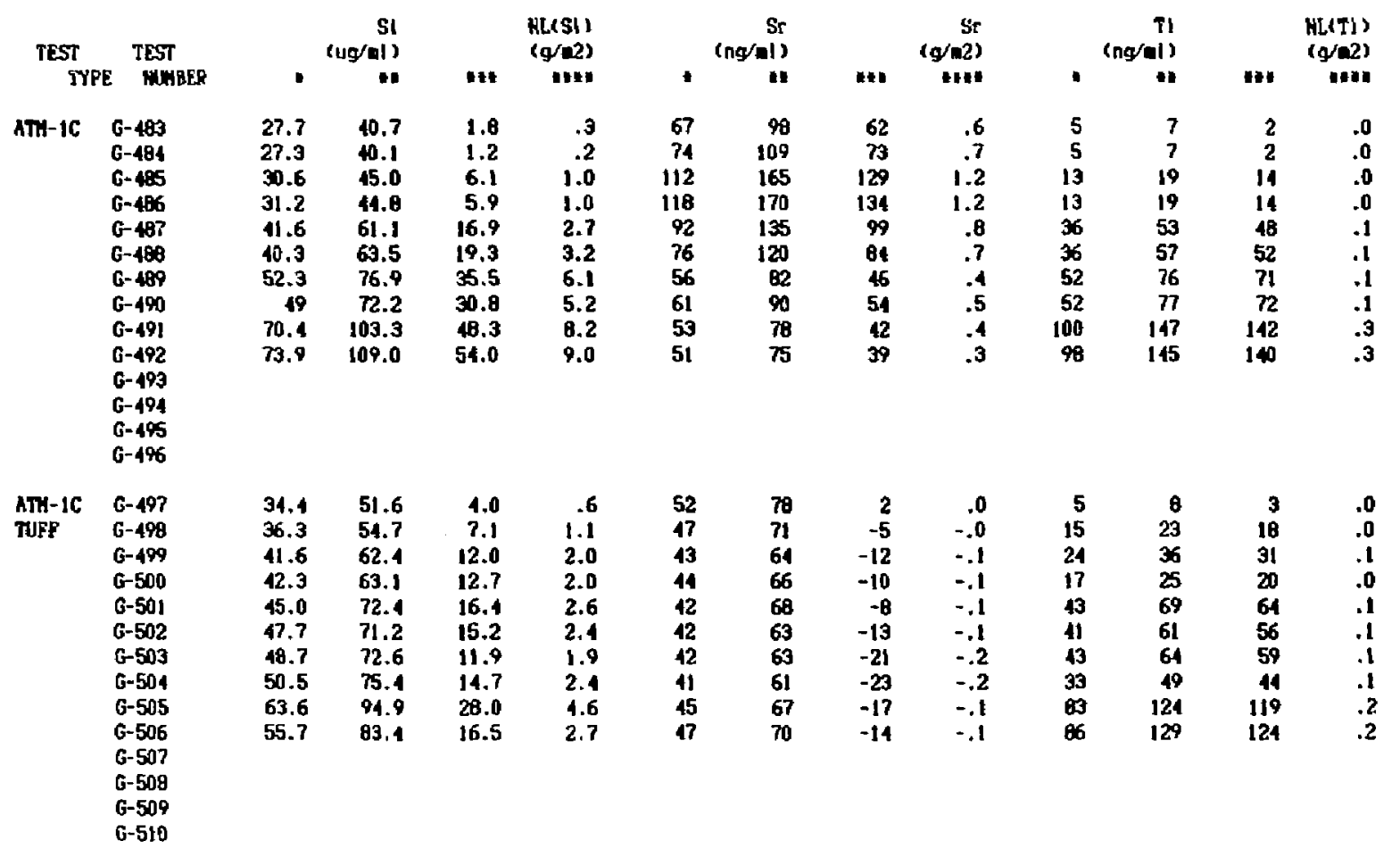


Table A-Ba (Cont'd)

\begin{tabular}{|c|c|c|c|c|c|c|c|c|c|c|c|c|c|}
\hline \multirow{2}{*}{$\begin{array}{l}\text { TEST } \\
\text { TYPE }\end{array}$} & \multirow{2}{*}{$\begin{array}{l}\text { TEST } \\
\text { MNABER }\end{array}$} & \multicolumn{2}{|c|}{$\begin{array}{r}\text { U } \\
(n g / a)\end{array}$} & \multicolumn{2}{|r|}{$\begin{array}{l}R L(U) \\
\left(g^{\prime}=2\right)\end{array}$} & \multicolumn{2}{|c|}{$\begin{array}{r}2 n \\
(n g / n)\end{array}$} & \multicolumn{2}{|c|}{$\begin{array}{l}A L(2 n) \\
(q / 2)\end{array}$} & \multicolumn{2}{|c|}{$\begin{array}{r}\text { Cs } \\
\left(n g / e^{\prime}\right)\end{array}$} & \multicolumn{2}{|r|}{$\begin{array}{l}\text { HL(Cg) } \\
(g / a 2)\end{array}$} \\
\hline & & - & 14 & 4 & 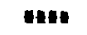 & - & 1 & 16 & twat & 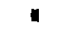 & $\|$ & ent & ини: \\
\hline$A T H-I C$ & $\begin{array}{l}G-489 \\
G-484 \\
G-485 \\
G-486 \\
G-487 \\
G-488 \\
G-489 \\
G-490 \\
G-491 \\
G-492 \\
G-493 \\
G-494 \\
G-495 \\
G-496\end{array}$ & $\begin{array}{r}72 \\
57 \\
165 \\
226 \\
480 \\
172 \\
520 \\
534 \\
937 \\
945\end{array}$ & $\begin{array}{r}106 \\
84 \\
244 \\
325 \\
705 \\
744 \\
764 \\
787 \\
1974 \\
1394\end{array}$ & $\begin{array}{r}106 \\
84 \\
244 \\
325 \\
705 \\
744 \\
764 \\
787 \\
1374 \\
1394\end{array}$ & $\begin{array}{l}.10 \\
.08 \\
.24 \\
.30 \\
.64 \\
.71 \\
.75 \\
.75 \\
1.34 \\
1.33\end{array}$ & $\begin{array}{l}200 \\
160 \\
130 \\
460 \\
1210 \\
1390 \\
1760 \\
1580 \\
3600 \\
3430\end{array}$ & $\begin{array}{l}294 \\
295 \\
632 \\
661 \\
1776 \\
2190 \\
2587 \\
2330 \\
5281 \\
5060\end{array}$ & $\begin{array}{l}271 \\
212 \\
609 \\
638 \\
1753 \\
2167 \\
2564 \\
2307 \\
5258 \\
5037\end{array}$ & $\begin{array}{l}.2 \\
.2 \\
.6 \\
.6 \\
1.5 \\
2.0 \\
2.4 \\
2.1 \\
4.8 \\
4.5\end{array}$ & $\begin{array}{l}170 \\
200 \\
530 \\
690\end{array}$ & $\begin{array}{r}250 \\
294 \\
779 \\
862 \\
0 \\
0 \\
0 \\
0 \\
0 \\
0\end{array}$ & $\begin{array}{r}250 \\
291 \\
779 \\
662 \\
0 \\
0 \\
0 \\
0 \\
0 \\
0\end{array}$ & $\begin{array}{l}.9 \\
1.1 \\
3.0 \\
3.2 \\
0.0 \\
0.0 \\
0.0 \\
0.0 \\
0.0 \\
0.0\end{array}$ \\
\hline $\begin{array}{l}\text { ATH - IC } \\
\text { TUFP }\end{array}$ & $\begin{array}{l}G-497 \\
G-498 \\
G-499 \\
G-500 \\
G-501 \\
G-502 \\
G-503 \\
G-504 \\
G-505 \\
G-506 \\
G-507 \\
G-508 \\
G-509 \\
G-510\end{array}$ & $\begin{array}{l}193 \\
288 \\
313 \\
339 \\
390 \\
427 \\
127 \\
439 \\
172 \\
499\end{array}$ & $\begin{array}{l}290 \\
434 \\
469 \\
497 \\
628 \\
637 \\
637 \\
617 \\
705 \\
748\end{array}$ & $\begin{array}{l}290 \\
434 \\
469 \\
497 \\
628 \\
637 \\
637 \\
647 \\
705 \\
748\end{array}$ & $\begin{array}{l}.27 \\
.40 \\
.44 \\
.45 \\
.57 \\
.59 \\
.59 \\
.61 \\
.66 \\
.70\end{array}$ & $\begin{array}{r}170 \\
200 \\
110 \\
420 \\
550 \\
650 \\
1170 \\
720 \\
1560 \\
2110\end{array}$ & $\begin{array}{r}255 \\
301 \\
615 \\
627 \\
685 \\
970 \\
1745 \\
1075 \\
2328 \\
3161\end{array}$ & $\begin{array}{r}211 \\
260 \\
574 \\
586 \\
844 \\
929 \\
1715 \\
1045 \\
2287 \\
3120\end{array}$ & $\begin{array}{r}.2 \\
.2 \\
.5 \\
.5 \\
.7 \\
.0 \\
1.5 \\
.9 \\
2.0 \\
2.8\end{array}$ & $\begin{array}{l}100 \\
120 \\
180 \\
130\end{array}$ & $\begin{array}{l}150 \\
181 \\
270 \\
194\end{array}$ & $\begin{array}{l}150 \\
181 \\
270 \\
194\end{array}$ & $\begin{array}{r}.5 \\
.7 \\
1.0 \\
.7\end{array}$ \\
\hline
\end{tabular}


Table A-8a (Cont'd)

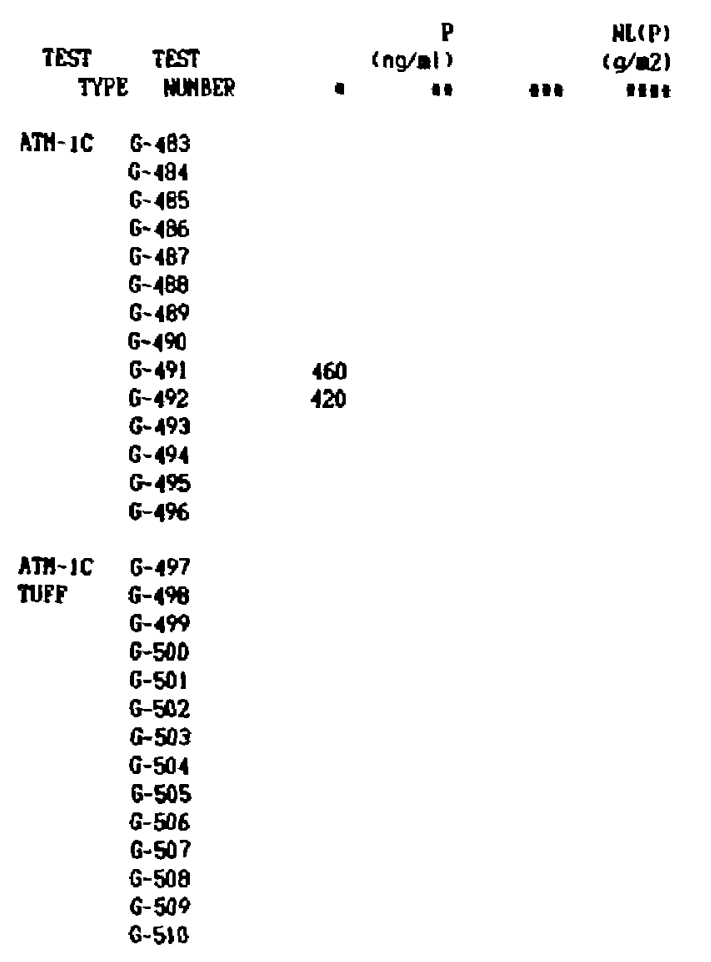


Table A-8a (Cont'd)

\begin{tabular}{|c|c|c|c|c|c|c|c|c|c|c|c|c|}
\hline \multirow{2}{*}{$\begin{array}{l}\text { TEST } \\
\text { TYPE }\end{array}$} & \multirow{2}{*}{$\begin{array}{l}\text { TEST } \\
\text { MWHBER }\end{array}$} & \multirow{2}{*}{$\begin{array}{r}\text { SNMPLE } \\
\text { SA } \\
\text { TOTAL } \\
\text { VESSEL }\end{array}$} & \multirow{2}{*}{$\begin{array}{r}1120 \\
\text { VOLLWE } \\
\text { IH }\end{array}$} & \multirow{2}{*}{$\begin{array}{c}\text { SOL'H } \\
\text { valu,18 }\end{array}$} & \multicolumn{2}{|c|}{$\begin{array}{r}\text { Al } \\
\text { (ng/at) }\end{array}$} & \multicolumn{2}{|r|}{$\begin{array}{l}\text { HL(AI) } \\
(g / \infty 2)\end{array}$} & \multicolumn{2}{|c|}{ (ng/mil) } & \multicolumn{2}{|r|}{$\begin{array}{c}M L(t) \\
(g / a z)\end{array}$} \\
\hline & & & & & 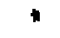 & 4 & nx & Hitu & " & 14 & unk & Inul \\
\hline$A T H-B C$ & $\begin{array}{l}6-511 \\
6-512 \\
G-513 \\
G-51 \\
G-515 \\
6-516 \\
6-517 \\
G-518 \\
6-519 \\
G-520 \\
G-521\end{array}$ & $\begin{array}{l}485 \\
477 \\
470 \\
492 \\
472 \\
499 \\
481 \\
479 \\
485 \\
475 \\
464\end{array}$ & $\begin{array}{l}14.87 \\
14.90 \\
14.87 \\
14.95 \\
14.90 \\
14.87 \\
14.91 \\
14.91 \\
14.91 \\
14.67 \\
14.94\end{array}$ & $\begin{array}{l}11.68 \\
12.39 \\
11.95 \\
12.49 \\
11.85 \\
12.01 \\
11.62 \\
12.21 \\
11.85 \\
12.35\end{array}$ & $\begin{array}{l}460 \\
480 \\
430 \\
430 \\
240 \\
230 \\
260 \\
220 \\
260 \\
270\end{array}$ & $\begin{array}{l}682 \\
674 \\
610 \\
602 \\
341 \\
326 \\
372 \\
310 \\
370 \\
379\end{array}$ & $\begin{array}{r}-43 \\
-51 \\
-115 \\
-123 \\
-384 \\
-399 \\
-296 \\
-360 \\
-355 \\
-346\end{array}$ & $\begin{array}{r}-.2 \\
-.3 \\
-.6 \\
-.6 \\
-2.1 \\
-2.1 \\
-1.6 \\
-1.9 \\
-1.9 \\
-1.9\end{array}$ & $\begin{array}{r}420 \\
470 \\
610 \\
700 \\
2760 \\
2130 \\
3620 \\
3660 \\
4070 \\
3940\end{array}$ & $\begin{array}{r}597 \\
660 \\
665 \\
960 \\
3925 \\
3017 \\
5178 \\
5159 \\
5787 \\
5535\end{array}$ & $\begin{array}{r}448 \\
511 \\
716 \\
631 \\
3776 \\
2868 \\
5029 \\
5010 \\
5638 \\
5306\end{array}$ & $\begin{array}{l}.5 \\
.6 \\
.9 \\
1.0 \\
1.6 \\
3.4 \\
6.0 \\
6.0 \\
6.7 \\
6.5\end{array}$ \\
\hline $\begin{array}{l}\text { ATI-B } \\
+ \text { TUFP }\end{array}$ & $\begin{array}{l}G-525 \\
G-526 \\
G-527 \\
G-528 \\
G-529 \\
G-530 \\
G-531 \\
G-532 \\
G-533 \\
G-534 \\
G-535 \\
G-536 \\
G-537 \\
G-536\end{array}$ & $\begin{array}{l}487 \\
492 \\
465 \\
486 \\
484 \\
506 \\
490 \\
493 \\
471 \\
477 \\
477 \\
489 \\
485\end{array}$ & $\begin{array}{l}14.49 \\
14.48 \\
14.59 \\
14.47 \\
14.47 \\
14.50 \\
14.50 \\
14.52 \\
14.50 \\
14.51 \\
14.55 \\
14.55 \\
14.54 \\
14.47\end{array}$ & $\begin{array}{r}11.40 \\
11.86 \\
11.46 \\
12.01 \\
11.06 \\
11.90 \\
11.19 \\
11.70 \\
11.3 \\
12.01\end{array}$ & $\begin{array}{l}240 \\
220 \\
130 \\
270 \\
210 \\
190 \\
190 \\
160 \\
190 \\
150\end{array}$ & $\begin{array}{l}315 \\
313 \\
187 \\
302 \\
297 \\
269 \\
275 \\
228 \\
271 \\
212\end{array}$ & $\begin{array}{r}81 \\
49 \\
-77 \\
118 \\
35 \\
5 \\
110 \\
63 \\
10 \\
-52\end{array}$ & $\begin{array}{r}.1 \\
.2 \\
-.1 \\
.6 \\
.2 \\
.0 \\
.6 \\
.3 \\
.1 \\
-.3\end{array}$ & $\begin{array}{r}940 \\
920 \\
2890 \\
4320 \\
1450 \\
4680 \\
1780 \\
5021 \\
6110 \\
5880\end{array}$ & $\begin{array}{l}1352 \\
1308 \\
1151 \\
6119 \\
6926 \\
6633 \\
6916 \\
7165 \\
6814 \\
6328\end{array}$ & $\begin{array}{l}1175 \\
1131 \\
3974 \\
5942 \\
6149 \\
6456 \\
6739 \\
6969 \\
8637 \\
8151\end{array}$ & $\begin{array}{r}1.3 \\
1.3 \\
4.8 \\
6.8 \\
7.1 \\
7.1 \\
7.7 \\
7.9 \\
10.2 \\
9.5\end{array}$ \\
\hline
\end{tabular}


Table A-8a (Cont'd)

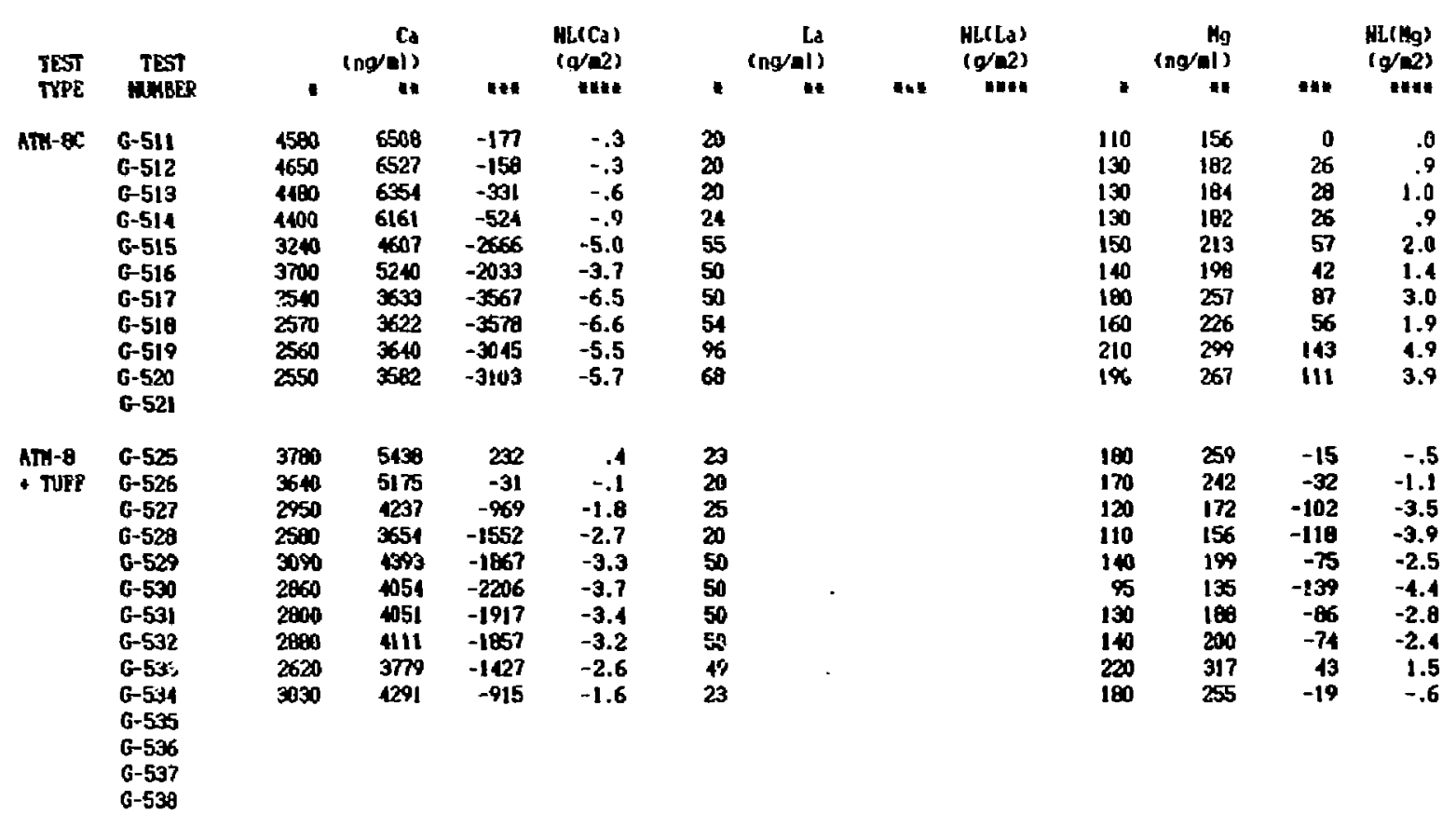


Table A-Ba (Cont'd)

\begin{tabular}{|c|c|c|c|c|c|c|c|c|c|c|c|c|c|}
\hline $\begin{array}{l}\text { TEST } \\
\text { TPEE }\end{array}$ & $\begin{array}{c}\text { TEST } \\
\text { MUEBR }\end{array}$ & • & $\begin{array}{r}\text { Ho } \\
9 / a 1) \\
=11\end{array}$ & " & $\begin{array}{l}\text { HL(Mo) } \\
(g / m 2) \\
n \in H\end{array}$ & * & $\begin{array}{r}\mathrm{Ma} \\
\mathrm{I} / \mathrm{al} \\
\mathbf{m}\end{array}$ & MIII & $\begin{array}{l}\text { HL(Ha) } \\
\left(g^{\prime}\left(\omega^{\prime}\right)\right. \\
\end{array}$ & " & $\begin{array}{r}\text { S! } \\
|9 / m|) \\
n !\end{array}$ & $\cdots$ & 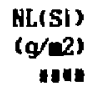 \\
\hline$A T H-B C$ & $\begin{array}{l}G-511 \\
G-512 \\
G-513 \\
G-514 \\
G-515 \\
G-516 \\
G-517 \\
G-518 \\
G-519 \\
G-520 \\
G-521\end{array}$ & $\begin{array}{c}150 \\
220 \\
280 \\
300 \\
1420 \\
1080 \\
1830 \\
1870 \\
2010 \\
1980\end{array}$ & $\begin{array}{l}213 \\
309 \\
397 \\
120 \\
2019 \\
15 ; 1 \\
2617 \\
2636 \\
2958 \\
2782\end{array}$ & $\begin{array}{r}163 \\
259 \\
347 \\
370 \\
1969 \\
1480 \\
2567 \\
2586 \\
2908 \\
2732\end{array}$ & $\begin{array}{r}.4 \\
.6 \\
.0 \\
.0 \\
4.4 \\
3.2 \\
5.5 \\
5.7 \\
6.3 \\
6.1\end{array}$ & $\begin{array}{l}38.70 \\
39.50 \\
39.20 \\
39.60 \\
47.40 \\
45.00 \\
49.80 \\
49.80 \\
53.10 \\
51.70\end{array}$ & $\begin{array}{l}55.0 \\
54.0 \\
55.6 \\
55.5 \\
67.1 \\
63.7 \\
71.2 \\
70.2 \\
75.5 \\
72.6\end{array}$ & $\begin{array}{r}4.1 \\
3.4 \\
5.0 \\
4.9 \\
16.8 \\
13.1 \\
20.6 \\
19.6 \\
24.9 \\
22.0\end{array}$ & $\begin{array}{l}1.4 \\
1.1 \\
1.7 \\
1.6 \\
5.7 \\
1.3 \\
6.5 \\
6.5 \\
8.2 \\
7.4\end{array}$ & $\begin{array}{l}29.4 \\
29.6 \\
29.8 \\
30.5 \\
39.0 \\
35.6 \\
39.8 \\
40.3 \\
48.3 \\
45.4\end{array}$ & $\begin{array}{l}41.1 \\
11.5 \\
42.3 \\
12.7 \\
54.0 \\
50.4 \\
56.9 \\
56.8 \\
68.7 \\
63.0\end{array}$ & $\begin{array}{r}2.2 \\
2.6 \\
3.4 \\
3.8 \\
9.8 \\
6.2 \\
16.0 \\
15.9 \\
27.8 \\
22.9\end{array}$ & $\begin{array}{l}.4 \\
.5 \\
.6 \\
.7 \\
1.8 \\
1.1 \\
2.8 \\
2.8 \\
4.8 \\
4.1\end{array}$ \\
\hline $\begin{array}{l}\text { ATH-8 } \\
+ \text { TUPF }\end{array}$ & $\begin{array}{l}G-525 \\
G-526 \\
G-527 \\
G-529 \\
G-529 \\
G-530 \\
G-531 \\
G-532 \\
G-539 \\
G-531 \\
G-535 \\
G-536 \\
G-537 \\
G-538\end{array}$ & $\begin{array}{r}420 \\
400 \\
1470 \\
2280 \\
2400 \\
2440 \\
2460 \\
2620 \\
3180 \\
3070\end{array}$ & $\begin{array}{r}604 \\
569 \\
2111 \\
3229 \\
3412 \\
3458 \\
3559 \\
3740 \\
1597 \\
4348\end{array}$ & $\begin{array}{r}554 \\
519 \\
2061 \\
3179 \\
3362 \\
3408 \\
3509 \\
3690 \\
1537 \\
1296\end{array}$ & $\begin{array}{l}1.2 \\
1.1 \\
4.6 \\
6.7 \\
7.1 \\
6.9 \\
7.4 \\
7.7 \\
9.9 \\
9.9\end{array}$ & $\begin{array}{l}37.30 \\
38.30 \\
42.40 \\
45.60 \\
48.50 \\
49.20 \\
47.90 \\
48.00 \\
49.90 \\
50.00\end{array}$ & $\begin{array}{l}53.7 \\
54.4 \\
60.9 \\
66.0 \\
68.9 \\
69.7 \\
69.3 \\
60.5 \\
72.0 \\
70.0\end{array}$ & $\begin{array}{r}2.8 \\
3.5 \\
10.0 \\
15.1 \\
19.0 \\
18.8 \\
19.9 \\
19.1 \\
21.1 \\
19.9\end{array}$ & $\begin{array}{l}.9 \\
1.1 \\
3.3 \\
4.8 \\
5.8 \\
5.8 \\
6.3 \\
6.0 \\
6.9 \\
6.5\end{array}$ & $\begin{array}{l}34.6 \\
36.9 \\
42.4 \\
44.4 \\
48.9 \\
17.9 \\
51.0 \\
5.7 \\
55.3 \\
55.8\end{array}$ & $\begin{array}{l}\mathbf{4 9 . 8} \\
52.5 \\
60.9 \\
62.9 \\
69.5 \\
67.9 \\
73.8 \\
73.8 \\
79.5 \\
04.7\end{array}$ & $\begin{array}{r}2.2 \\
1.9 \\
10.5 \\
12.5 \\
19.5 \\
11.9 \\
13.0 \\
13.0 \\
19.0 \\
23.9\end{array}$ & $\begin{array}{l}.4 \\
.8 \\
1.9 \\
2.1 \\
2.3 \\
1.9 \\
2.2 \\
2.2 \\
3.3 \\
1.1\end{array}$ \\
\hline
\end{tabular}


Table A-Ba (Cont'd)

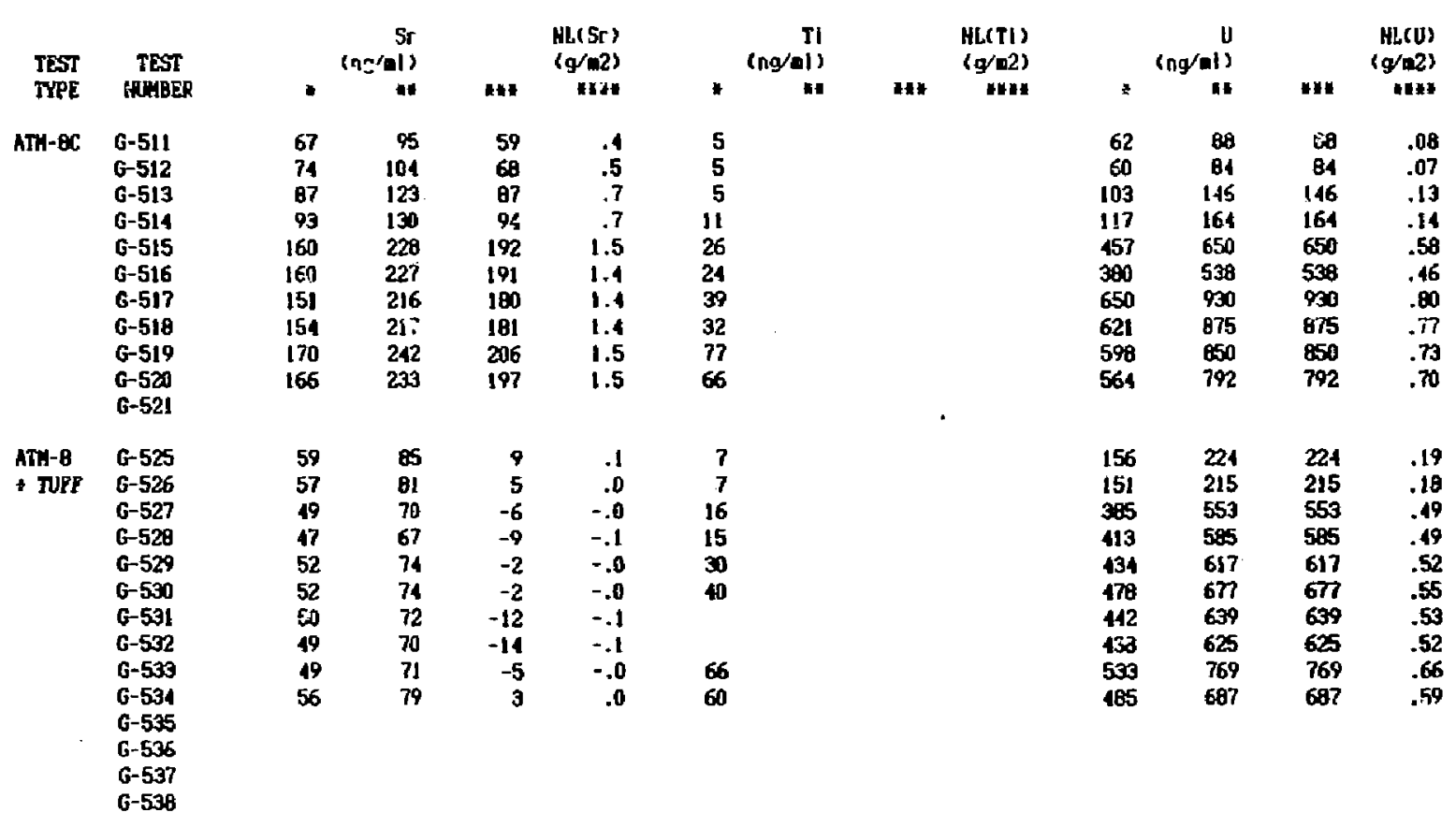


Table A-8e (Cont'd)

\begin{tabular}{|c|c|c|c|c|c|c|c|c|c|}
\hline TEST & TEST & & $\begin{array}{r}2 n \\
9 a 1)\end{array}$ & & $\begin{array}{l}H L(2 n) \\
\left(g / q^{\prime} 2\right)\end{array}$ & & $\begin{array}{r}\text { Cs } \\
\text { (al) }\end{array}$ & & $\begin{array}{l}\text { HLe(Cs) } \\
(g / n 2)\end{array}$ \\
\hline TYPE & HUHBER & - & $n$ & $m$ & Han & " & 14 & 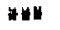 & mar \\
\hline ATH- $8 C$ & $\begin{array}{l}G-511 \\
G-512 \\
G-513 \\
G-514 \\
G-515 \\
G-516 \\
G-517 \\
G-516 \\
G-519 \\
G-520 \\
G-521\end{array}$ & $\begin{array}{r}140 \\
160 \\
240 \\
300 \\
730 \\
570 \\
1210 \\
1110 \\
1890 \\
1800\end{array}$ & $\begin{array}{r}199 \\
225 \\
349 \\
420 \\
1038 \\
607 \\
1731 \\
1565 \\
2687 \\
2529\end{array}$ & $\begin{array}{r}176 \\
202 \\
317 \\
397 \\
1015 \\
784 \\
1708 \\
1542 \\
2654 \\
2506\end{array}$ & $\begin{array}{r}.1 \\
.2 \\
.3 \\
.3 \\
.9 \\
.7 \\
1.4 \\
1.3 \\
2.3 \\
2.2\end{array}$ & $\begin{array}{l}130 \\
140 \\
190 \\
220\end{array}$ & $\begin{array}{r}185 \\
196 \\
269 \\
308 \\
0 \\
0 \\
0 \\
0 \\
0 \\
0\end{array}$ & $\begin{array}{r}185 \\
190 \\
269 \\
308 \\
0 \\
0 \\
0 \\
0 \\
0 \\
0\end{array}$ & $\begin{array}{l}.6 \\
.7 \\
.9 \\
1.0 \\
0.0 \\
0.0 \\
0.0 \\
0.0 \\
0.0 \\
0.0\end{array}$ \\
\hline $\begin{array}{l}\text { ATH-O } \\
+ \text { TUPE }\end{array}$ & $\begin{array}{l}G-525 \\
G-526 \\
G-527 \\
G-528 \\
G-529 \\
G-530 \\
G-531 \\
G-532 \\
G-533 \\
G-531 \\
G-535 \\
G-536 \\
G-537 \\
G-5338\end{array}$ & $\begin{array}{r}170 \\
160 \\
310 \\
260 \\
580 \\
830 \\
690 \\
730 \\
1420 \\
1150\end{array}$ & $\begin{array}{r}245 \\
227 \\
445 \\
397 \\
625 \\
1176 \\
998 \\
1042 \\
2048 \\
1629\end{array}$ & $\begin{array}{r}204 \\
186 \\
404 \\
356 \\
784 \\
1135 \\
957 \\
1001 \\
2007 \\
1588\end{array}$ & $\begin{array}{r}.2 \\
.2 \\
.3 \\
.3 \\
.6 \\
.9 \\
.8 \\
.8 \\
1.7 \\
1.3\end{array}$ & $\begin{array}{r}160 \\
40 \\
110 \\
130\end{array}$ & $\begin{array}{r}230 \\
57 \\
158 \\
184\end{array}$ & $\begin{array}{r}230 \\
57 \\
159 \\
184\end{array}$ & $\begin{array}{l}.8 \\
.2 \\
.5 \\
.6\end{array}$ \\
\hline
\end{tabular}


Table A-8a (Cont'd)

TEST

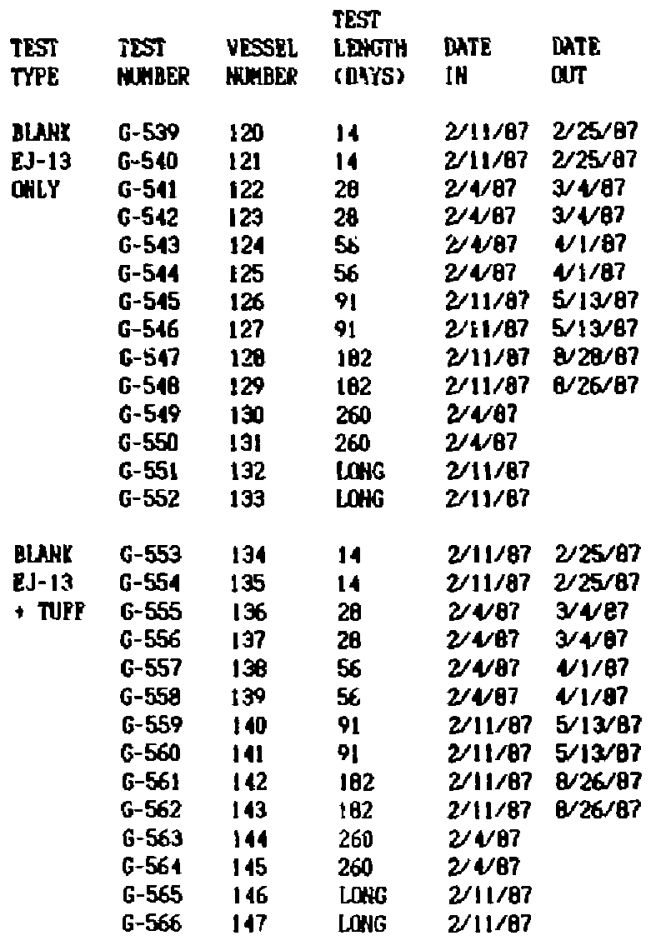

H20 COHPLETE CONPLETE VOLARE ASSDABLY ASSEABLY

IH IH OUT

16.22 300.62 $16.20 \quad 303.22 \quad 303.23$ $16.18 \quad 295.41 \quad 295.43$

$\begin{array}{llll}6.20 & 297.89 & 297.91\end{array}$

$\begin{array}{lll}16.19 & 300.95 & 300.97\end{array}$

$16.25 \quad 300.28 \quad 300.30$

$\begin{array}{lll}16.14 & 298.65 \quad 298.65\end{array}$

$\begin{array}{lll}16.20 & 299.18 & 299.19\end{array}$

$\begin{array}{lll}16.19 & 298.59 & 298.61\end{array}$

16.20 $303.37 \quad 303.37$

$16.16 \quad 297.36$

$16.23 \quad 299.20$

$16.21 \quad 299.23$

$16.21 \quad 304.92$

15. $45 \quad 300.21 \quad 30.122$

$\begin{array}{lll}15.44 & 305.65 & 305.68\end{array}$

$\begin{array}{lll}15.49 & 305.82 & 305.86\end{array}$

$\begin{array}{llll}15.45 & 300.01 & 300.03\end{array}$

$\begin{array}{llll}15.49 & 298.91 & 290.94\end{array}$

$15.46 \quad 304.71 \quad 304.7$

$\begin{array}{lll}15.54 & 309.40 & 300.42\end{array}$

$\begin{array}{llll}15.46 & 303.74 & 303.75\end{array}$

$\begin{array}{lll}15.53 & 298.22 & 298.24\end{array}$

$15.48 \quad 299.26$

15.5301 .01

$15.47 \quad 300.91$

$15.57 \quad 301.50$
DELTA PH LPE LPE SOL'H CARBOH

HASS OUT TARE HEIGH VOLUHE PPH

$-.02$

.02

24.21

23.66

24.34

24.51

23.68

23.12

23.69

24.57

$\begin{array}{llllll}0.00 & 8.01 & 23.65 & 35.73 & 12.08 & \mathbf{4 5 . 7 6}\end{array}$

36.37

36. 81

12.16

31.63

$12.17 \quad 31.50$

36.73

$\begin{array}{lll}35.8 & 12.12 & 35.7\end{array}$

$\begin{array}{lll}53.18 & 12.06 & 31.58\end{array}$

$\begin{array}{lll}35.85 & 12.16 & 30.2\end{array}$

$\begin{array}{lll}35.74 & 12.17 \quad 43.53\end{array}$

$$
\begin{array}{rr}
-.01 & 7.71 \\
-.01 & 7.62 \\
-.03 & 7.43 \\
-.04 & 7.23 \\
-.02 & 7.33 \\
-.03 & 7.35 \\
-.01 & 7.5 \\
-.02 & 7.43 \\
-.01 & 7.49 \\
-.02 & 7.37 \\
299.26 & \\
301.01 & \\
300.91 & \\
301.50 &
\end{array}
$$$$
-.02 \quad 7.33
$$

$\begin{array}{rrrr}24.54 & 27.27 & 2.79 & 32.79 \\ 24.05 & 35.39 & 11.34 & 33.50 \\ 23.70 & 34.84 & 11.14 & 36.89 \\ 24.49 & 35.85 & 11.37 & 12.35 \\ 24.55 & 35.71 & 11.16 & 39.33 \\ 23.95 & 35.29 & 11.29 & 39.43 \\ 24.25 & 35.56 & 11.31 & 37.68 \\ 23.99 & 35.33 & 11.34 & 34.62 \\ 24.21 & 35.49 & 11.28 & 43.85 \\ 24.52 & 35.93 & 11.41 & 43.41\end{array}$


Table A-8a (Cont'd)

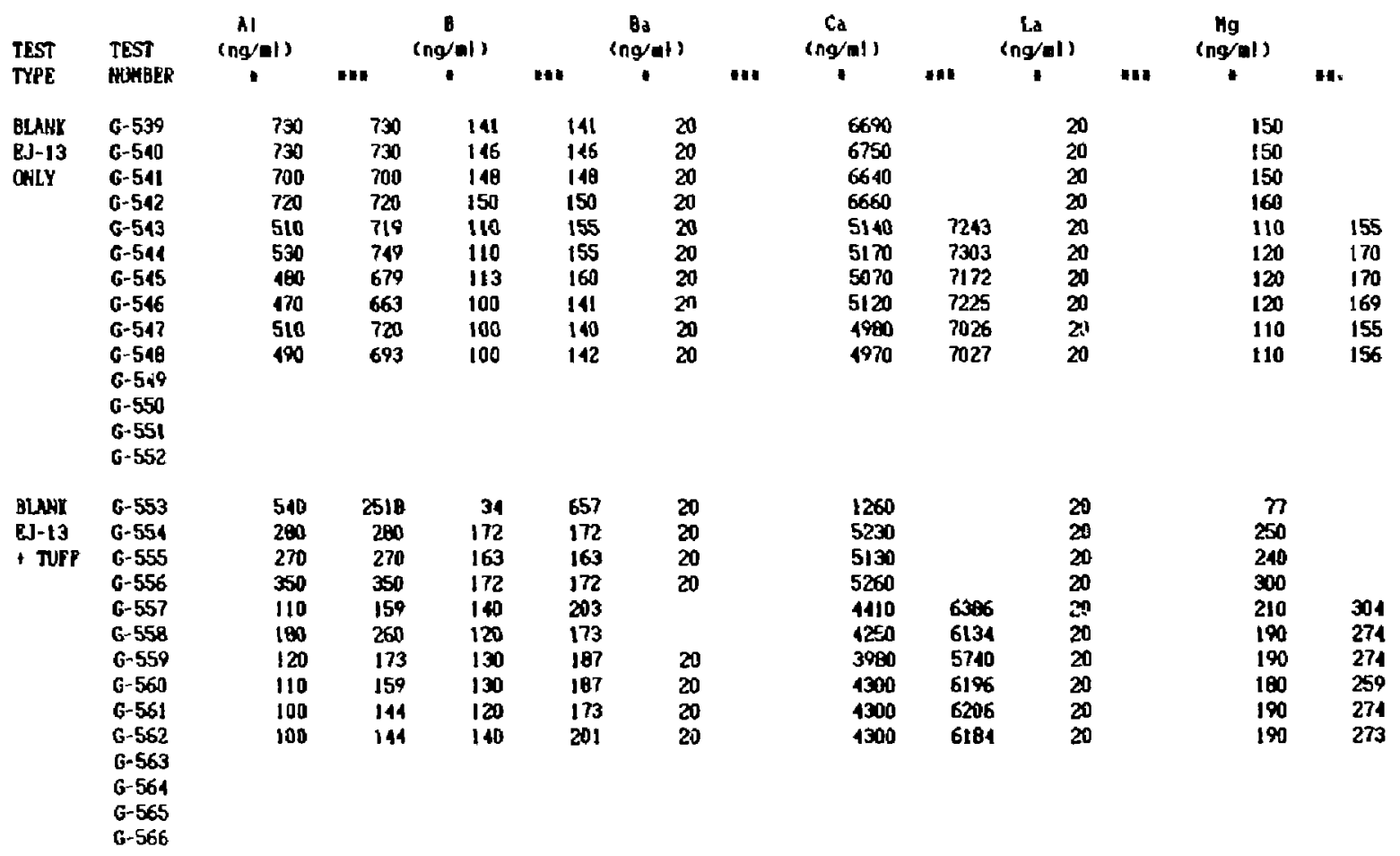


Table A-8a (Cont'd)

\begin{tabular}{|c|c|c|c|c|c|c|c|c|c|c|c|c|c|c|}
\hline $\begin{array}{l}\text { TEST } \\
\text { TVPE }\end{array}$ & $\begin{array}{l}\text { TEST } \\
\text { HUABER }\end{array}$ & $\begin{array}{c}\mathrm{Ho}_{0} \\
(\mathrm{ng} / \mathrm{01}) \\
!\end{array}$ & III & $\underset{(u g / m 1)}{H a}$ & W" & $\underset{(u g / a l)}{S i}$ & W" & $\begin{array}{c}\mathrm{Sr} \\
(\mathrm{ng} / \mathrm{m} /) \\
!\end{array}$ & Mn & $\begin{array}{c}T i \\
\left(n g^{\prime}(m+1)\right.\end{array}$ & int & $\underset{(n g / m)}{2 n}$ & $m$ & $\underset{(n g / m i 1)}{2 r}$ \\
\hline $\begin{array}{l}\text { DLAHX } \\
\text { Ey-13 } \\
\text { OWLY }\end{array}$ & $\begin{array}{l}G-539 \\
G-540 \\
G-541 \\
G-512 \\
G-543 \\
G-514 \\
G-545 \\
G-546 \\
G-547 \\
G-548 \\
G-549 \\
G-558 \\
G-551 \\
G-552\end{array}$ & $\begin{array}{l}50 \\
50 \\
50 \\
50 \\
50 \\
50 \\
50 \\
50 \\
50 \\
50\end{array}$ & & $\begin{array}{r}50.20 \\
50.10 \\
50.20 \\
50.50 \\
36.00 \\
36.60 \\
35.40 \\
35.70 \\
34.9 \\
34.9\end{array}$ & $\begin{array}{l}50.7 \\
52.0 \\
50.1 \\
50.4 \\
49.2 \\
49.3\end{array}$ & $\begin{array}{l}38.5 \\
38.9 \\
39.7 \\
38.5 \\
31.5 \\
31.1 \\
30.1 \\
27.8 \\
38.3 \\
39.6\end{array}$ & $\begin{array}{l}44.4 \\
43.9 \\
42.6 \\
39.2 \\
54.0 \\
56.0\end{array}$ & $\begin{array}{l}36 \\
36 \\
36 \\
36 \\
26 \\
26 \\
27 \\
27 \\
26 \\
26\end{array}$ & $\begin{array}{l}37 \\
37 \\
30 \\
38 \\
37 \\
37\end{array}$ & $\begin{array}{l}5 \\
5 \\
5 \\
5 \\
5 \\
5 \\
5 \\
5 \\
5 \\
5\end{array}$ & & $\begin{array}{l}23 \\
29 \\
24 \\
28 \\
16 \\
16 \\
21 \\
15 \\
12 \\
12\end{array}$ & $\begin{array}{l}23 \\
23 \\
30 \\
21 \\
17 \\
17\end{array}$ & $\begin{array}{l}20 \\
20 \\
20 \\
20 \\
20 \\
20 \\
20 \\
20 \\
20 \\
20\end{array}$ \\
\hline $\begin{array}{l}\text { BLANK } \\
\text { EJ-13 } \\
+ \text { TUPP }\end{array}$ & $\begin{array}{l}6-553 \\
6-554 \\
G-555 \\
G-556 \\
G-55 ? \\
G-550 \\
G-559 \\
G-560 \\
G-561 \\
G-562 \\
G-563 \\
G-564 \\
G-565 \\
G-566\end{array}$ & $\begin{array}{l}50 \\
50 \\
50 \\
50 \\
50 \\
50 \\
50 \\
50 \\
50 \\
50\end{array}$ & & $\begin{array}{r}10.90 \\
50.50 \\
50.70 \\
50.60 \\
35.30 \\
35.60 \\
34.20 \\
34.30 \\
34.3 \\
34.4\end{array}$ & $\begin{array}{r}51.1 \\
51.7 \\
49.3 \\
49.1 \\
99.5 \\
49.5\end{array}$ & $\begin{array}{r}11.3 \\
47.6 \\
49.3 \\
51.5 \\
38.8 \\
38.6 \\
41.5 \\
42.6 \\
46 \\
46.8\end{array}$ & $\begin{array}{l}56.2 \\
55.7 \\
59.8 \\
61.7 \\
66.4 \\
67.3\end{array}$ & $\begin{array}{l}18 \\
74 \\
73 \\
73 \\
59 \\
58 \\
55 \\
61 \\
60 \\
57\end{array}$ & $\begin{array}{l}85 \\
84 \\
79 \\
88 \\
97 \\
82\end{array}$ & $\begin{array}{l}5 \\
5 \\
5 \\
5 \\
5 \\
5 \\
5 \\
5 \\
5 \\
5\end{array}$ & & $\begin{array}{l}80 \\
45 \\
48 \\
52 \\
33 \\
27 \\
31 \\
30 \\
10 \\
22\end{array}$ & $\begin{array}{l}46 \\
39 \\
45 \\
43 \\
26 \\
32\end{array}$ & $\begin{array}{l}20 \\
20 \\
20 \\
20 \\
20 \\
20 \\
20 \\
20 \\
20 \\
20\end{array}$ \\
\hline
\end{tabular}




\section{TABLE A-8b}

Cation analyses from the OR (silicone gasket) experiments. 
Table A-8b, Solution Rosulte for Proliminery ATH-1c Experimente Dene without Redietion

\begin{tabular}{|c|c|c|c|c|c|c|c|c|c|c|c|c|}
\hline \multirow[b]{2}{*}{ Toet } & \multirow[b]{2}{*}{$\begin{array}{l}\text { Pength } \\
\text { Deye }\end{array}$} & \multirow[b]{2}{*}{ SA } & \multirow[b]{2}{*}{$\begin{array}{l}\text { Solution } \\
\text { Volume. }\end{array}$} & \multicolumn{2}{|c|}{ Al } & \multicolumn{3}{|c|}{ B } & \multicolumn{2}{|c|}{$c \bullet$} & \multicolumn{2}{|c|}{ ول } \\
\hline & & & & $\underset{\text { PPD }}{E J-13}$ & $\begin{array}{c}\text { Solut! ton } \\
\text { ppon }\end{array}$ & $\underset{\text { PPD }}{E J-13}$ & $\begin{array}{l}\text { Solution" } \\
\text { ppm }\end{array}$ & NL & $\underset{P P=}{E J-13}$ & $\begin{array}{l}\text { Solution } \\
\text { DPD }\end{array}$ & $\begin{array}{c}\text { EJ-13 } \\
\text { PPD }\end{array}$ & $\begin{array}{l}\text { Solution } \\
\text { PPa }\end{array}$ \\
\hline $\begin{array}{l}G-476 \\
G-4 \\
G-4 . j \\
G-486 \\
G-478 \\
G-482 \\
c-477 \\
c-461\end{array}$ & $\begin{array}{l}7 \\
74 \\
14 \\
28 \\
28 \\
63 \\
63\end{array}$ & $\begin{array}{l}474.7 \\
405.2 \\
491.5 \\
492.8 \\
408.9 \\
498.3 \\
479.5 \\
406.1\end{array}$ & & $\begin{array}{r}6.25 \\
6.25 \\
6.25 \\
6.25 \\
6.25 \\
6.25 \\
6.25\end{array}$ & $\begin{array}{l}0.13 \\
0.13 \\
6.16 \\
3.16 \\
6.14 \\
6.10 \\
6.16 \\
0.20\end{array}$ & $\begin{array}{r}0.16 \\
0.16 \\
0.25 \\
6.25 \\
. .25 \\
6.15 \\
. .16 \\
. .16\end{array}$ & $\begin{array}{r}7.54 \\
7.28 \\
12.92 \\
12.36 \\
17.22 \\
16.75 \\
29.75 \\
20.76\end{array}$ & $\begin{array}{r}7.7 \\
7.2 \\
13.2 \\
12.8 \\
17.6 \\
17.3 \\
a 2.8 \\
30.6\end{array}$ & $\begin{array}{l}7.12 \\
7.12 \\
7.12 \\
7.12 \\
7.12 \\
7.12 \\
7.12 \\
7.12\end{array}$ & $\begin{array}{l}6.2 \\
6.4 \\
3.1 \\
3.1 \\
1.7 \\
1.9 \\
0.7 \\
0.8\end{array}$ & $\begin{array}{l}0.27 \\
0.27 \\
0.27 \\
0.27 \\
6.27 \\
6.27 \\
6.27 \\
0.27\end{array}$ & $\begin{array}{l}0.22 \\
0.21 \\
0.17 \\
0.23 \\
. .24 \\
6.28 \\
. .32 \\
. .31\end{array}$ \\
\hline
\end{tabular}

\begin{tabular}{|c|c|c|c|c|c|c|c|c|c|c|c|}
\hline \multicolumn{3}{|c|}{ He } & \multicolumn{3}{|c|}{ si } & \multicolumn{3}{|c|}{ Sr } & \multicolumn{3}{|c|}{$\mathbf{u}$} \\
\hline EJ-23 & $\begin{array}{c}\text { Solution } \\
\text { ppin }\end{array}$ & $\mathbf{M L}$ & $\underset{\text { PPM }}{E J-13}$ & $\begin{array}{l}\text { Solution } \\
\text { Ppe }\end{array}$ & ML. & $\underset{\mathrm{PPm}}{\mathrm{EJ}-13}$ & $\begin{array}{c}\text { Solution } \\
\text { ppen }\end{array}$ & NH. & $\underset{\text { Ppe }}{E J-12}$ & $\begin{array}{l}\text { Solution } \\
\text { ppe }\end{array}$ & NHL \\
\hline $\begin{array}{r}5.4 \\
48.4 \\
48.4 \\
48.4 \\
96.4 \\
96.4 \\
48.4 \\
48.4\end{array}$ & $\begin{array}{r}85.8 \\
67.2 \\
78.8 \\
77.8 \\
08.4 \\
88.8 \\
114.8 \\
113.4\end{array}$ & $\begin{array}{r}0.6 \\
0.0 \\
11.4 \\
10.0 \\
13.7 \\
14.5 \\
24.9 \\
24.0\end{array}$ & $\begin{array}{l}96.3 \\
95.3 \\
85.3 \\
85.3 \\
85.3 \\
95.3 \\
96.3 \\
85.3\end{array}$ & $\begin{array}{l}48.2 \\
49.8 \\
69.1 \\
67.1 \\
02.5 \\
65.5 \\
92.5 \\
88.9\end{array}$ & $\begin{array}{l}1.6 \\
2.6 \\
8.0 \\
2.7 \\
3.2 \\
9.6 \\
5.0 \\
0.6\end{array}$ & 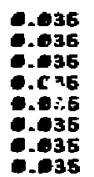 & $\begin{array}{r}0.24 \\
6.24 \\
6.19 \\
.19 \\
.12 \\
.13 \\
. .06 \\
.06\end{array}$ & $\begin{array}{r}1.4 \\
1.4 \\
1.4 \\
1.0 \\
6.0 \\
6.2 \\
0.2\end{array}$ & $\begin{array}{l}8 \\
8 \\
8\end{array}$ & $\begin{array}{r}6.63 \\
6.08 \\
6.63 \\
6.61 \\
0.71 \\
0.67 \\
1.11 \\
1.64\end{array}$ & $\begin{array}{r}0.46 \\
6.48 \\
6.52 \\
6.51 \\
6.58 \\
6.56 \\
6.83 \\
9.86\end{array}$ \\
\hline
\end{tabular}

The raperted velues eppeared to be low, based on intultion, and the solutions are beling reanalyzed. 


\section{TABLE A-9}

$\mathrm{PH}$ and Anion Analyses for the 2R Experiments

This Table presents the $\mathrm{pH}$ and anion results for the $2 \mathrm{R}$ experiments. For each anion, the concentration in the undiluted leachate in $\mathrm{pPm}$ is given together with a blank corrected value. The blank correction is made by surtracting the values obtained in the blank experiments from the analogous experiments containing glass. If this subtraction results in a negative number, the results are given in parenthesis. 
Table A-8. pH and Anion Analysos - Blank Samples

\begin{tabular}{|c|c|c|c|c|c|c|c|c|c|c|c|c|c|}
\hline \multirow[b]{3}{*}{ Tast Iype } & \multirow[b]{3}{*}{ tegt } & \multirow[b]{3}{*}{$\begin{array}{l}\text { Langlh } \\
\text { (Days) }\end{array}$} & \multirow[b]{3}{*}{ Dut } & \multicolumn{8}{|c|}{ Concenteation (wpw, $t / \mathrm{g} / \mathrm{at})$} & \multirow{3}{*}{ 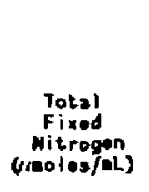 } & \multirow[b]{3}{*}{$\begin{array}{c}\text { Blank } \\
\text { Corrected } \\
\text { wrooles/mi }\end{array}$} \\
\hline & & & & \multirow{2}{*}{\multicolumn{2}{|c|}{$\frac{F^{-}}{\begin{array}{c}\text { Olank } \\
\text { Corrected }\end{array}}$}} & \multirow{2}{*}{\multicolumn{2}{|c|}{$\frac{\mathrm{Cl}-}{\begin{array}{c}\text { Blank } \\
\text { corrected }\end{array}}$}} & \multicolumn{2}{|r|}{$\mathrm{se}_{4}=$} & \multirow[b]{2}{*}{$\mathrm{MO}_{3^{-}}$} & \multirow[b]{2}{*}{$\mathrm{wos}^{-}$} & & \\
\hline & & & & & & & & & $\begin{array}{l}\text { Blank } \\
\text { Correctod }\end{array}$ & & & & \\
\hline $\begin{array}{l}\text { ATU-1C } \\
\text { J-is }\end{array}$ & $\begin{array}{l}51 \\
52 \\
49 \\
51 \\
63 \\
54 \\
66 \\
88\end{array}$ & $\begin{array}{l}7 \\
7 \\
14 \\
14 \\
28 \\
28 \\
68 \\
68\end{array}$ & $\begin{array}{l}7.33 \\
7.23 \\
7.74 \\
7.20 \\
7.60 \\
7.63 \\
7.24 \\
7.28\end{array}$ & $\begin{array}{l}2.65 \\
2.80 \\
2.40 \\
2.40 \\
2.45 \\
2.75 \\
2.76 \\
2.76\end{array}$ & $\begin{array}{l}(0.16) \\
(.15) \\
(.76) \\
(.25) \\
(0.156 \\
(0.35 \\
(1.35)\end{array}$ & $\begin{array}{r}7.60 \\
7.65 \\
7.60 \\
7.40 \\
7.45 \\
6.65\end{array}$ & 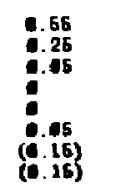 & $\begin{array}{l}21.8 \\
18.0 \\
19.3 \\
19.8 \\
20.1 \\
17.6 \\
10.8\end{array}$ & 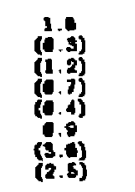 & $\begin{array}{l}4.84 \\
3.95 \\
3.05 \\
3.60 \\
2.85 \\
2.95 \\
4.80 \\
6.80\end{array}$ & $\begin{array}{l}3.30 \\
3.10 \\
6.04 \\
5.54 \\
7.94 \\
8.94 \\
7.85\end{array}$ & $\begin{array}{r}0.14 \\
6.14 \\
8.10 \\
8.20 \\
6.22 \\
8.25\end{array}$ & $\begin{array}{l}(0.05) \\
(6.05) \\
(8.01) \\
(6.05) \\
(8.43) \\
(8.87)\end{array}$ \\
\hline $\begin{array}{l}\text { And-1c } \\
\text { J-is } \\
\text { ruif }\end{array}$ & $\begin{array}{l}68 \\
80 \\
67 \\
68 \\
81 \\
82 \\
83 \\
84\end{array}$ & $\begin{array}{l}7 \\
7 \\
14 \\
14 \\
28 \\
28 \\
58 \\
58\end{array}$ & $\begin{array}{l}7.22 \\
7.39 \\
7.43 \\
7.38 \\
7.47 \\
7.33 \\
7.10 \\
7.68\end{array}$ & $\begin{array}{l}2.40 \\
2.10 \\
2.20 \\
2.65 \\
2.55 \\
2.74 \\
2.94 \\
2.76\end{array}$ & $\begin{array}{l}(8.2) \\
(8.4) \\
(8.05) \\
(8.20) \\
(8.15) \\
(0.3)\end{array}$ & $\begin{array}{l}7.80 \\
7.80 \\
8.85 \\
7.95 \\
7.25 \\
7.45 \\
8.15\end{array}$ & $\begin{array}{l}8.80 \\
8.86 \\
848 \\
8.90 \\
8.60\end{array}$ & $\begin{array}{l}21.8 \\
19.8 \\
18.3 \\
22.8 \\
28.8 \\
21.8 \\
22.4 \\
21.8\end{array}$ & $\begin{array}{l}2.3 \\
(0.30) \\
(2.80) \\
1.10 \\
1.20 \\
1.20 \\
1.80 \\
0.20\end{array}$ & $\begin{array}{l}3.65 \\
8.15 \\
4.70 \\
6.15 \\
2.85 \\
3.70 \\
5.10 \\
3.76\end{array}$ & $\begin{array}{l}3.70 \\
3.35 \\
4.65 \\
4.85 \\
8.80 \\
7.10 \\
6.10 \\
8.10\end{array}$ & $\begin{array}{r}8.14 \\
6.17 \\
6.18 \\
6.19 \\
6.21 \\
8.25\end{array}$ & 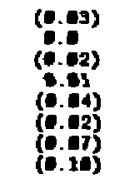 \\
\hline 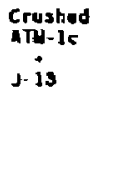 & $\begin{array}{l}87 \\
88 \\
66 \\
80 \\
80 \\
70 \\
71 \\
72\end{array}$ & $\begin{array}{r}7 \\
7 \\
14 \\
14 \\
29 \\
28 \\
58 \\
68\end{array}$ & $\begin{array}{l}7.41 \\
8.95 \\
7.08 \\
7.01 \\
7.34 \\
7.30 \\
7.28\end{array}$ & $\begin{array}{l}2.45 \\
2.46 \\
2.55 \\
2.05 \\
2.75 \\
2.75 \\
3.45 \\
2.85\end{array}$ & $\begin{array}{l}(8.20) \\
(8.20) \\
(0.10) \\
(0.05) \\
(0.05) \\
(0.25)\end{array}$ & $\begin{array}{l}7.05 \\
7.05 \\
7.94 \\
9.15 \\
7.41 \\
7.65 \\
8.65 \\
8.15\end{array}$ & $\begin{array}{c}(8.20) \\
(8.20) \\
8.40 \\
8.05 \\
8.10 \\
8.95 \\
(0.15)\end{array}$ & $\begin{array}{l}19.3 \\
19.8 \\
21.1 \\
21.8 \\
20.4 \\
29.3 \\
21.9 \\
21.2\end{array}$ & $\begin{array}{l}0.1 \\
0.4 \\
1.4 \\
1.2 \\
1.1 \\
1.4 \\
0.7\end{array}$ & $\begin{array}{l}0.16 \\
4.85 \\
2.15 \\
2.15 \\
2.15 \\
2.75 \\
3.46 \\
3.31\end{array}$ & $\begin{array}{c}3.35 \\
4.16 \\
6.96 \\
6.85 \\
7.85 \\
7.80 \\
10.1 \\
8.96\end{array}$ & $\begin{array}{r}0.17 \\
6.10 \\
6.19 \\
6.21 \\
6.21 \\
6.27\end{array}$ & 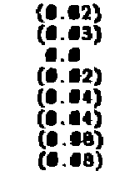 \\
\hline & & & & & & & Cont'd & & & & & & \\
\hline
\end{tabular}


Iasle A-9 (Cont'd)

\begin{tabular}{|c|c|c|c|c|c|c|c|c|c|c|c|c|c|}
\hline \multirow[b]{2}{*}{ Tost Type } & \multirow{2}{*}{ lost } & \multirow{2}{*}{$\begin{array}{l}\text { Lenglh } \\
\text { (Dyys) }\end{array}$} & \multirow{2}{*}{ out } & \multicolumn{2}{|r|}{$F^{-}$} & \multicolumn{2}{|r|}{ Cl. } & \multicolumn{2}{|r|}{$\mathrm{SO}_{4} \mathrm{n}$} & \multirow{2}{*}{$\frac{\mathrm{NO}_{3}^{*}}{\mathrm{MCL}}$} & \multirow{2}{*}{$\frac{\mathrm{ma}_{2}^{-}}{\mathrm{ACL}}$} & \multirow{2}{*}{$\begin{array}{c}\text { Totol } \\
\text { Fixed } \\
\text { Mitrogen } \\
\text { Guolegelol }\end{array}$} & \multirow{2}{*}{$\begin{array}{c}\text { Olank } \\
\text { Corractao } \\
\text { Goroles/al }\end{array}$} \\
\hline & & & & ACL & Corrected & ACL & Corracted & $\mathrm{ACL}$ & Corracted & & & & \\
\hline $\begin{array}{l}\text { ATL-: } \\
1-13\end{array}$ & $\begin{array}{l}75 \\
70 \\
73 \\
74 \\
717 \\
70 \\
70\end{array}$ & $\begin{array}{l}7 \\
7 \\
14 \\
14 \\
21 \\
21 \\
58 \\
68\end{array}$ & 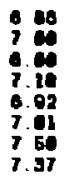 & $\begin{array}{l}2.64 \\
2.75 \\
2.65 \\
2.4 \\
2.40 \\
3.16 \\
3.14 \\
3.14\end{array}$ & $\begin{array}{l}(0.05) \\
6.25) \\
6.10 \\
6.35\end{array}$ & $\begin{array}{l}7.25 \\
7.05 \\
7.98 \\
7.65 \\
7.60 \\
7.65 \\
7.68\end{array}$ & $\begin{array}{r}8.20) \\
6.20 \\
0.10 \\
6.24 \\
(6.85)\end{array}$ & $\begin{array}{l}20.2 \\
20.2 \\
21.8 \\
31.9 \\
28.5 \\
28.5 \\
20.6 \\
20.7\end{array}$ & $\begin{array}{l}1: \\
1: 1 \\
1.1 \\
1.5 \\
1.3 \\
6.2\end{array}$ & $\begin{array}{l}7.10 \\
6.55 \\
6.95 \\
5.48 \\
1.10 \\
4.10 \\
1.10 \\
6.00\end{array}$ & $\begin{array}{l}3.15 \\
3.30 \\
3.35 \\
3.70 \\
7.16 \\
7.65 \\
9.15 \\
9.10\end{array}$ & $\begin{array}{r}0.18 \\
0.16 \\
0.16 \\
6.17 \\
6.22 \\
6.23 \\
6.29\end{array}$ & $\begin{array}{l}(0.01) \\
(0.01) \\
(0.01) \\
(0.02) \\
(6.03) \\
(0.02) \\
(0.04) \\
(0.05)\end{array}$ \\
\hline Jufis & $\begin{array}{l}03 \\
04 \\
01 \\
02 \\
05 \\
80 \\
e t \\
a b\end{array}$ & $\begin{array}{l}7 \\
7 \\
14 \\
14 \\
28 \\
20 \\
68 \\
56\end{array}$ & $\begin{array}{l}7.6 \\
7.28 \\
7.28 \\
7.23 \\
7.24 \\
7.41 \\
7.62\end{array}$ & $\begin{array}{l}2.05 \\
2.84 \\
2.85 \\
2.05 \\
2.45 \\
2.06 \\
3.10 \\
3.16\end{array}$ & 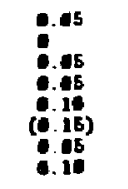 & $\begin{array}{l}7.26 \\
7.35 \\
7.38 \\
7.36 \\
7.20 \\
7.85 \\
7.86\end{array}$ & 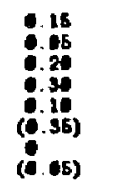 & $\begin{array}{l}20.4 \\
21.2 \\
21.4 \\
23.0 \\
24.2 \\
14.9 \\
21.8 \\
21.6\end{array}$ & $\begin{array}{l}0.0 \\
8.7 \\
0.1 \\
0.6 \\
6.7 \\
0.4\end{array}$ & $\begin{array}{l}6.60 \\
5.06 \\
5.45 \\
5.76 \\
2.36 \\
2.10 \\
5.65 \\
\text { s.65 }\end{array}$ & $\begin{array}{l}3.44 \\
3.65 \\
1.15 \\
4.35 \\
7.45 \\
6.00 \\
9.04 \\
0.10\end{array}$ & $\begin{array}{r}6.17 \\
6.17 \\
6.11 \\
6.10 \\
6.20 \\
6.20 \\
6.20\end{array}$ & $\begin{array}{c}0.4 \\
8.81) \\
8.1 \\
(8.43) \\
(8.45)\end{array}$ \\
\hline $\begin{array}{l}\text { Crushod } \\
\text { ATU- } \\
\text { J-is }\end{array}$ & $\begin{array}{l}91 \\
92 \\
89 \\
90 \\
83 \\
81 \\
85 \\
05\end{array}$ & $\begin{array}{r}7 \\
7 \\
14 \\
14 \\
28 \\
28 \\
56 \\
56\end{array}$ & $\begin{array}{l}0.80 \\
0.86 \\
7.10 \\
7.49 \\
7.37 \\
7.12 \\
7.14 \\
7.00\end{array}$ & $\begin{array}{l}2.85 \\
2.85 \\
2.76 \\
2.70 \\
3.40 \\
2.05 \\
3.20 \\
3.25\end{array}$ & $\begin{array}{l}6 \\
6.05 \\
0.15 \\
6.2 \\
6.16 \\
.15\end{array}$ & 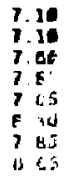 & 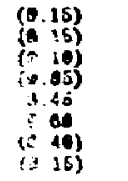 & $\begin{array}{l}19.6 \\
19.6 \\
28.9 \\
20.9 \\
21.2 \\
20.9 \\
18.9 \\
21.3\end{array}$ & $\begin{array}{r}0.1 \\
0.1 \\
6.4 \\
2.0 \\
1.1 \\
(0.2) \\
(0.2)\end{array}$ & $\begin{array}{l}5.60 \\
4.30 \\
1.91 \\
7.9 \\
3.10 \\
2.10 \\
3.46 \\
3.90\end{array}$ & $\begin{array}{r}3.90 \\
4.10 \\
5.76 \\
1.20 \\
0.45 \\
0.60 \\
12.5 \\
11.8\end{array}$ & $\begin{array}{r}0.17 \\
6.16 \\
6.24 \\
6.24 \\
6.20 \\
6.26 \\
0.33 \\
0.32\end{array}$ & $\begin{array}{l}(0.02) \\
(0.07) \\
: .1 \\
: .1 \\
0.1 \\
0.0 \\
(0.1)\end{array}$ \\
\hline
\end{tabular}


Iable A-9 (Cont"d)

\begin{tabular}{|c|c|c|c|c|c|c|c|c|c|}
\hline \multirow[b]{2}{*}{ Test Typs } & \multirow[b]{2}{*}{ Test } & \multirow[b]{2}{*}{$\begin{array}{l}\text { Length } \\
\text { (0sys) }\end{array}$} & \multirow[b]{2}{*}{$\begin{array}{c}\text { ph } \\
\text { out }\end{array}$} & \multicolumn{5}{|c|}{ Concentration (pqu, $\mu_{q} / \mathrm{al}$ ) } & \multirow{2}{*}{$\begin{array}{l}\text { Total Fined } \\
\text { Mitrogen } \\
\text { Uinol/foL) }\end{array}$} \\
\hline & & & & $\mathbf{F}^{-}$ & $\mathrm{Cl}^{-}$ & $\mathrm{SO}_{1}=$ & $\mathrm{wO}_{3^{-}}$ & $\mathrm{NO}_{2}-$ & \\
\hline $\begin{array}{l}\text { Blank } \\
\text { Wo Iuft }\end{array}$ & $\begin{array}{r}90 \\
109 \\
97 \\
98 \\
101 \\
102 \\
103 \\
104\end{array}$ & $\begin{array}{r}7 \\
7 \\
14 \\
14 \\
20 \\
28 \\
50 \\
68\end{array}$ & $\begin{array}{l}6.78 \\
0.68 \\
0.31 \\
8.48 \\
7.45 \\
7.15 \\
0.48 \\
0.11\end{array}$ & $\begin{array}{l}2.65 \\
2.65 \\
2.65 \\
2.60 \\
2.48 \\
2.40 \\
3.16 \\
3.45\end{array}$ & $\begin{array}{r}7.10 \\
7.35 \\
7.65 \\
7.46 \\
7.15 \\
7.65 \\
.35 \\
.05\end{array}$ & $\begin{array}{l}18.8 \\
10.7 \\
20.9 \\
28.1 \\
28.7 \\
10.7 \\
20.4 \\
20.8\end{array}$ & $\begin{array}{l}0.35 \\
7.20 \\
6.26 \\
8.70 \\
11.8 \\
0.15 \\
13.8 \\
12.8\end{array}$ & $\begin{array}{l}3.38 \\
3.18 \\
2.05 \\
2.05 \\
3.45 \\
1.35 \\
5.80 \\
0.85\end{array}$ & $\begin{array}{r}1 \\
0.10 \\
.18 \\
.20 \\
.28 \\
.24 \\
.34 \\
.35\end{array}$ \\
\hline $\begin{array}{l}\text { Blank } \\
\text { Tuff }\end{array}$ & $\begin{array}{l}107 \\
108 \\
115 \\
160 \\
110 \\
111 \\
112\end{array}$ & $\begin{array}{l}7 \\
7 \\
14 \\
14 \\
28 \\
20 \\
68 \\
68\end{array}$ & $\begin{array}{l}6.88 \\
6.94 \\
8.94 \\
6.84 \\
7.27 \\
7.45 \\
0.71 \\
0.61\end{array}$ & $\begin{array}{l}2.80 \\
2.06 \\
2.85 \\
2.68 \\
2.76 \\
2.75 \\
3.10 \\
3.40\end{array}$ & $\begin{array}{l}7.10 \\
7.10 \\
7.45 \\
7.65 \\
7.40 \\
7.78 \\
7.85 \\
7.45\end{array}$ & $\begin{array}{l}19.4 \\
10.8 \\
28.8 \\
28.0 \\
10.4 \\
18.7 \\
21.1 \\
20.1\end{array}$ & $\begin{array}{l}4.85 \\
4.15 \\
5.10 \\
3.95 \\
6.16 \\
4.01 \\
6.15 \\
4.10\end{array}$ & $\begin{array}{l}4.45 \\
6.20 \\
5.10 \\
4.20 \\
8.45 \\
0.90 \\
8.16\end{array}$ & $\begin{array}{r}0.17 \\
.16 \\
.19 \\
.18 \\
.22 \\
.23 \\
.35 \\
.31\end{array}$ \\
\hline Avarage & & $\begin{array}{l}7 \\
14 \\
28 \\
58\end{array}$ & $\begin{array}{l}0.71 \\
0.41 \\
7.10 \\
0.00\end{array}$ & $\begin{array}{l}2.65 \\
2.85 \\
2.80 \\
3.10\end{array}$ & $\begin{array}{r}7.25 \\
7.65 \\
7.40 \\
7.20\end{array}$ & $\begin{array}{l}19.2 \\
20.5 \\
19.2 \\
20.5\end{array}$ & & & $\begin{array}{r}0.19 \\
\quad .19 \\
6.25 \\
0.36\end{array}$ \\
\hline Tuff & & $\begin{array}{l}7 \\
14 \\
28 \\
58\end{array}$ & $\begin{array}{l}0.01 \\
0.89 \\
7.30 \\
0.06\end{array}$ & $\begin{array}{l}2.00 \\
2.60 \\
2.75 \\
3.15\end{array}$ & $\begin{array}{l}7.16 \\
7.56 \\
7.56 \\
7.65\end{array}$ & $\begin{array}{r}19.5 \\
25.9 \\
10.8 \\
28.8\end{array}$ & & & $\begin{array}{r}0.17 \\
8.18 \\
8.23 \\
0.33\end{array}$ \\
\hline
\end{tabular}


TABLE A-10

FY 1986 Anion

This iable presents the anion results for the $1 R$ and $O R$ ( $S i$ gaskets) experiments. The column annotations are:

* ACL reported results

** dilution corrected values

$\star \star \star$ blank corrected results 


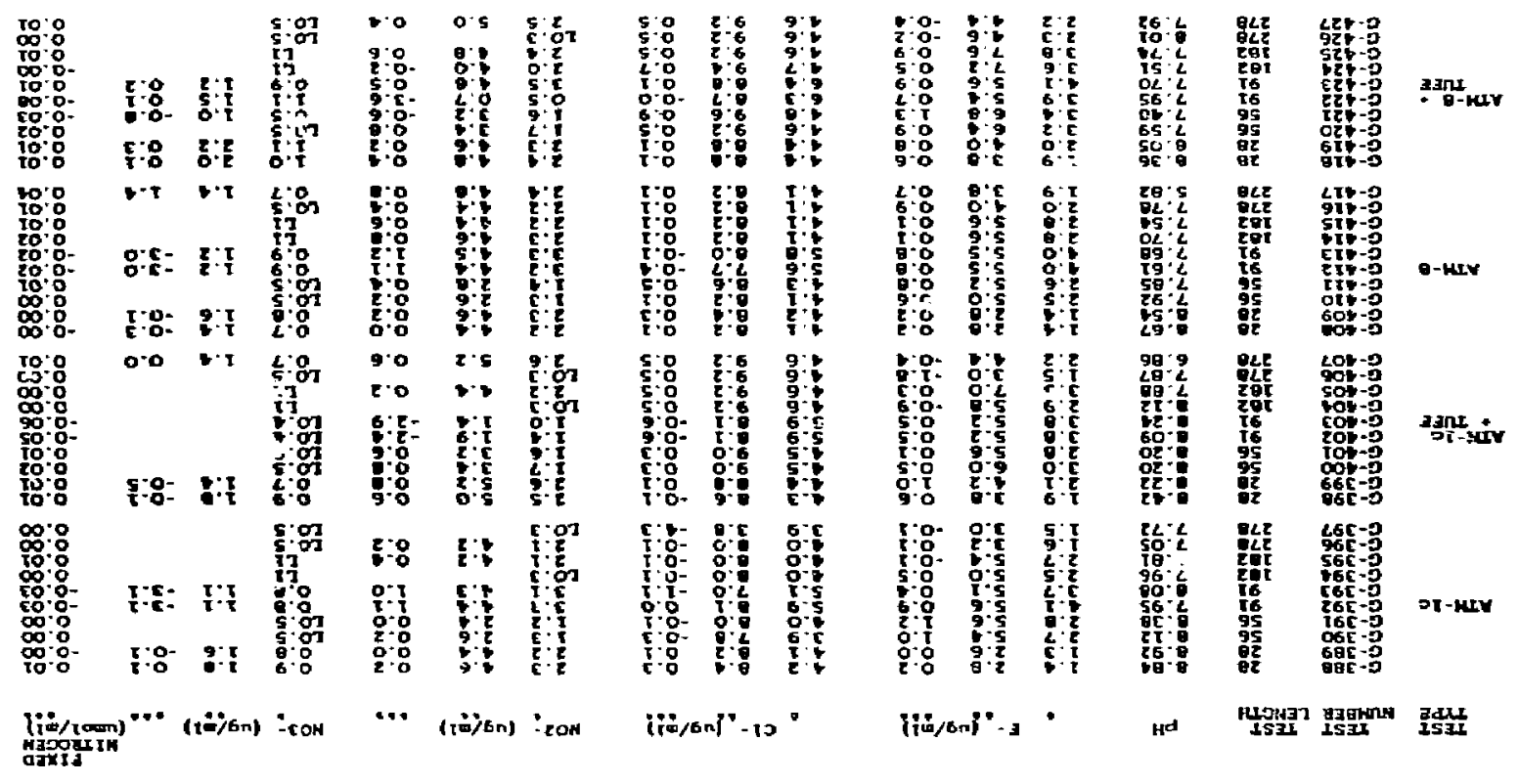

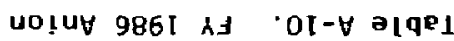




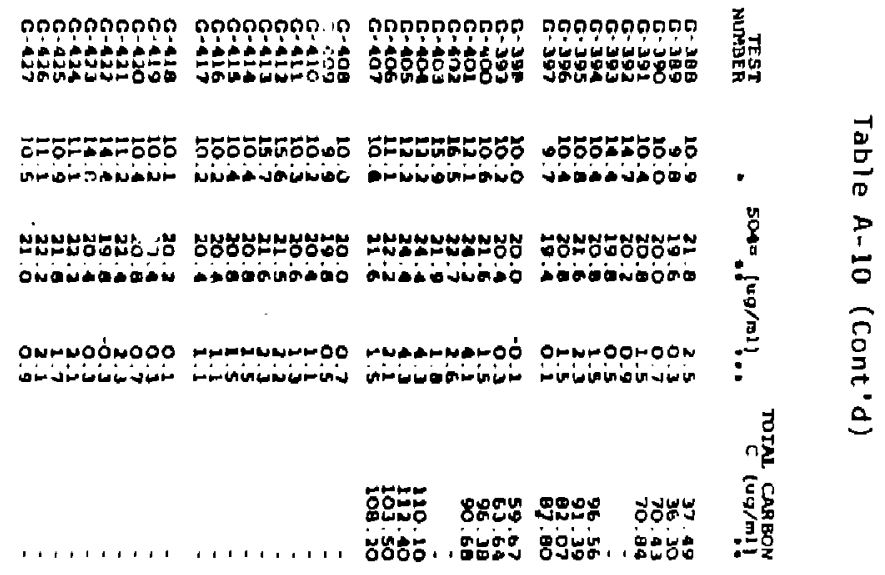


Table A-10 (Cont'd)

\begin{tabular}{|c|c|c|c|c|c|c|c|c|c|c|c|c|c|c|}
\hline EXP'T & $\begin{array}{c}\text { EXP.T } \\
\text { MUMBER }\end{array}$ & & PH & & $F-.(u g / m b)$ & & $c-g^{\mu}$ & $(g / \mathrm{a} 1)$ & Nax: & 11 & & NO3: & & \\
\hline EJ-13 & 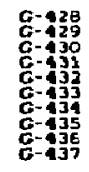 & $\begin{array}{r}28 \\
28 \\
56 \\
56 \\
91 \\
91 \\
981 \\
182 \\
182 \\
278 \\
278\end{array}$ & $\begin{array}{l}7.41 \\
3: 39 \\
6.57 \\
6.75 \\
6 \cdot 26 \\
5.54 \\
6.51 \\
6.79 \\
6.91 \\
6.98\end{array}$ & $\begin{array}{l}1: 3 \\
1: 3 \\
2: 2 \\
2: 2 \\
3: 2 \\
3: 2 \\
2: 8 \\
2: 7 \\
1: 6 \\
1: 5\end{array}$ & 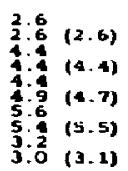 & $\begin{array}{r}4: 2 \\
4: 2 \\
3: 9 \\
6: 2 \\
16: 2 \\
4: 0 \\
3: 9 \\
4: 1 \\
4: 1\end{array}$ & $\begin{array}{r}8: 4 \\
8: 4 \\
7: 8 \\
7: 0 \\
8: 5 \\
22: 6 \\
8: 0 \\
7: 8 \\
8: 2 \\
8: 2\end{array}$ & $\begin{array}{l}(a .1) \\
(a .1) \\
(0.1) \\
(0.1) \\
(0.1)\end{array}$ & $\begin{array}{r}2: 2 \\
2: 2 \\
1: 2 \\
1: 2 \\
\frac{2}{2}: 2 \\
20: \frac{1}{1} \\
1: 7 \\
1: 1 \\
1: 0 \\
1: 0\end{array}$ & $\begin{array}{l}1: 4 \\
2: 1 \\
2: 1 \\
3: 3 \\
3.4 \\
1: 2 \\
4: 0\end{array}$ & $\begin{array}{l}(4.4) \\
(2.4) \\
(3.3) \\
(3.8) \\
(4.0)\end{array}$ & 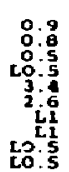 & $\begin{array}{r}2.8 \\
2: 6 \\
1.0 \\
4.7 \\
3.6\end{array}$ & $\begin{array}{r}(1.7) \\
(1.0) \\
(1.2) \\
\text { (0) } \\
\text { (0) }\end{array}$ \\
\hline$-13 \vec{R}$ & 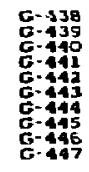 & 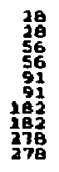 & $\begin{array}{l}3.03 \\
3.05 \\
6.41 \\
6.37 \\
6.70 \\
6.71 \\
6.63 \\
6.84 \\
6.81 \\
6.80\end{array}$ & $\begin{array}{l}1.6 \\
1.5 \\
2: 8 \\
2: 7 \\
3.5 \\
3.5 \\
3.5 \\
3.1 \\
2.3 \\
2.5\end{array}$ & $\begin{array}{l}3.2 \\
3: 2(3.2) \\
5: 6 \\
5: 5(5.5) \\
1: 8 \\
7.5(4.7) \\
7.2 \\
6.2(6.7) \\
5.6 \\
5.6(4.6)\end{array}$ & $\begin{array}{r}1.4 \\
1.3 \\
6.3 \\
5.6 \\
5.6 \\
4.3 \\
1.2\end{array}$ & 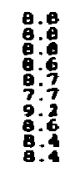 & $\begin{array}{l}(0.7) \\
(0.7) \\
(0.7) \\
(0.7) \\
(0.7)\end{array}$ & $\begin{array}{r}1: 5 \\
3: 0 \\
3: 2 \\
1: 9 \\
1: 3 \\
2: 4 \\
2: 2\end{array}$ & 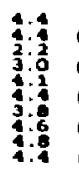 & $\begin{array}{l}(4.4) \\
(2.6) \\
(4.3) \\
(4.2) \\
(4.6)\end{array}$ & $\begin{array}{r}0.9 \\
1: 0 \\
1: 0 \\
0: 8 \\
1: 2 \\
0: 0 \\
4 \\
\text { ti. } \\
20.5 \\
0.7\end{array}$ & $\begin{array}{l}1: 0 \\
2: 0 \\
3: 0 \\
12: 5 \\
2: 2 \\
1.4\end{array}$ & $\begin{array}{r}(1.9) \\
(1.6) \\
(1.1) \\
(0) \\
(1.4)\end{array}$ \\
\hline Ho CJ-13 & $\begin{array}{l}G-148 \\
G-149 \\
G-450 \\
G-451 \\
G-452 \\
G-453 \\
G-454 \\
G-455 \\
G-156 \\
G-157 \\
G-158 \\
G-459\end{array}$ & $\begin{array}{l}14 \\
14 \\
28 \\
28 \\
26 \\
56 \\
56 \\
91 \\
91 \\
182 \\
182 \\
278 \\
278\end{array}$ & 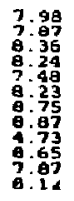 & $\begin{array}{l}1: 3 \\
1: 3 \\
1: 3 \\
1: 3 \\
1: 5 \\
1: 4 \\
1: 3 \\
1: 3 \\
1:-7 \\
1: 4 \\
1: 5 \\
1.4\end{array}$ & 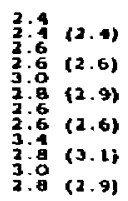 & $\begin{array}{r}1: 0 \\
3: 9 \\
4: 2 \\
1: 1 \\
3: 9 \\
3: 8 \\
3: 9 \\
31: 0 \\
14: 1 \\
26: 0 \\
20: 9\end{array}$ & $\begin{array}{r}9.0 \\
7: 8 \\
8: 8 \\
8: 2 \\
7: 8 \\
3: 6 \\
7.6 \\
7.6 \\
62: 2 \\
28: 2 \\
52: 0 \\
11.6\end{array}$ & $\begin{array}{l}(0.1) \\
(0.1) \\
(0.1) \\
(0.1) \\
(0.1) \\
(0.1)\end{array}$ & $\begin{array}{l}20.3 \\
20: 3 \\
20: 3 \\
20: 3 \\
60: 3 \\
60: 3 \\
20: 3 \\
20: 3 \\
20.2\end{array}$ & $\begin{array}{l}\text { (0) } \\
\text { (0) } \\
\text { (0) } \\
\text { (0) } \\
\text { (0) } \\
\text { (0) }\end{array}$ & & $\begin{array}{r}5: 1 \\
2: 4 \\
2: 1 \\
2: 7 \\
3: 7 \\
3: 8 \\
3: 9 \\
3: 9\end{array}$ & $\begin{array}{r}7.6 \\
7: 8 \\
70: 8 \\
4: 8 \\
5: 2 \\
5: 8 \\
7.6 \\
7.6 \\
7.6\end{array}$ & $\begin{array}{l}(7.5) \\
(0.5) \\
(4.6) \\
(5.4) \\
(7.7) \\
(7.6)\end{array}$ \\
\hline 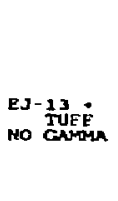 & 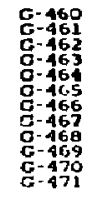 & $\begin{array}{r}14 \\
14 \\
28 \\
28 \\
56 \\
56 \\
91 \\
91 \\
182 \\
192 \\
278 \\
278\end{array}$ & $\begin{array}{l}7.27 \\
7: 34 \\
8.06 \\
7: 90 \\
7.10 \\
7.62 \\
8: 35 \\
8: 17 \\
7.98 \\
8: 12 \\
8.09 \\
8.02\end{array}$ & $\begin{array}{l}1: 2 \\
1: 2 \\
1: 4 \\
1: 4 \\
1: 3 \\
1: 3 \\
1: 4 \\
1: 2 \\
1.2 \\
1.5 \\
1.5\end{array}$ & 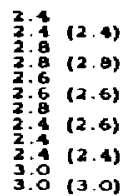 & $4: 1$ & 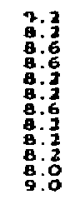 & $\begin{array}{l}(0.4) \\
(0.4) \\
(0.4) \\
(\theta .4) \\
(8.4) \\
(0.4)\end{array}$ & $\begin{array}{l}L 0: 3 \\
L O: 3 \\
L O: 3 \\
L O: 3 \\
L O: 3 \\
L 0: 3 \\
L 0: 3 \\
L 0: 3\end{array}$ & $\begin{array}{l}\text { (o) } \\
\text { (o) } \\
\text { (o) } \\
\text { (o) } \\
\text { (o) } \\
\text { (o) }\end{array}$ & & $\begin{array}{r}4: 0 \\
4: 1 \\
3: 2 \\
2: 5 \\
3: 4 \\
2: 7 \\
3: 6 \\
3: 7\end{array}$ & $\begin{array}{l}0.8 \\
7: 0 \\
9: 0 \\
9: 2 \\
5: 0 \\
6: 8 \\
5: 4 \\
7: 2 \\
7: 9 \\
7.0\end{array}$ & $\begin{array}{l}(7.9) \\
(2.1) \\
(4.3) \\
(6.1) \\
(7.3) \\
(7.9)\end{array}$ \\
\hline
\end{tabular}


Table A-10 (Cont'd)

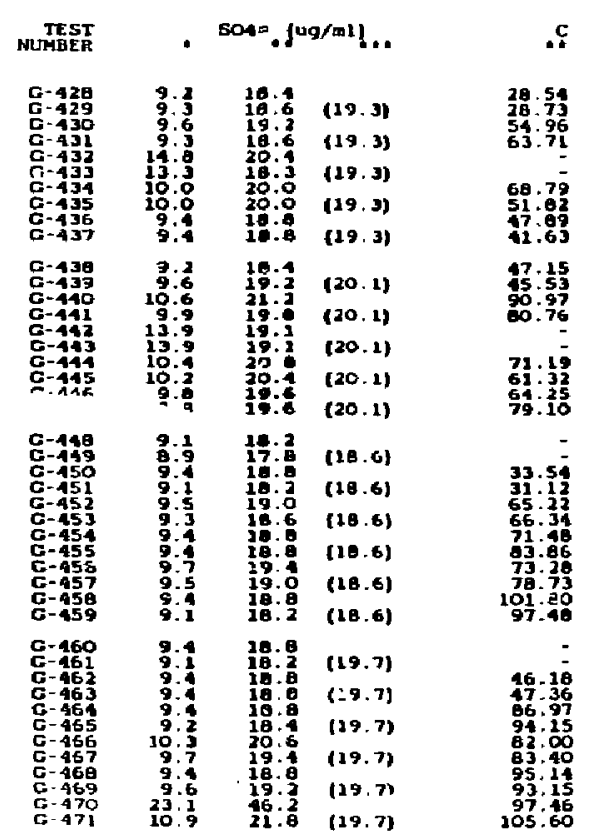


Table A-10 (Cont'd)

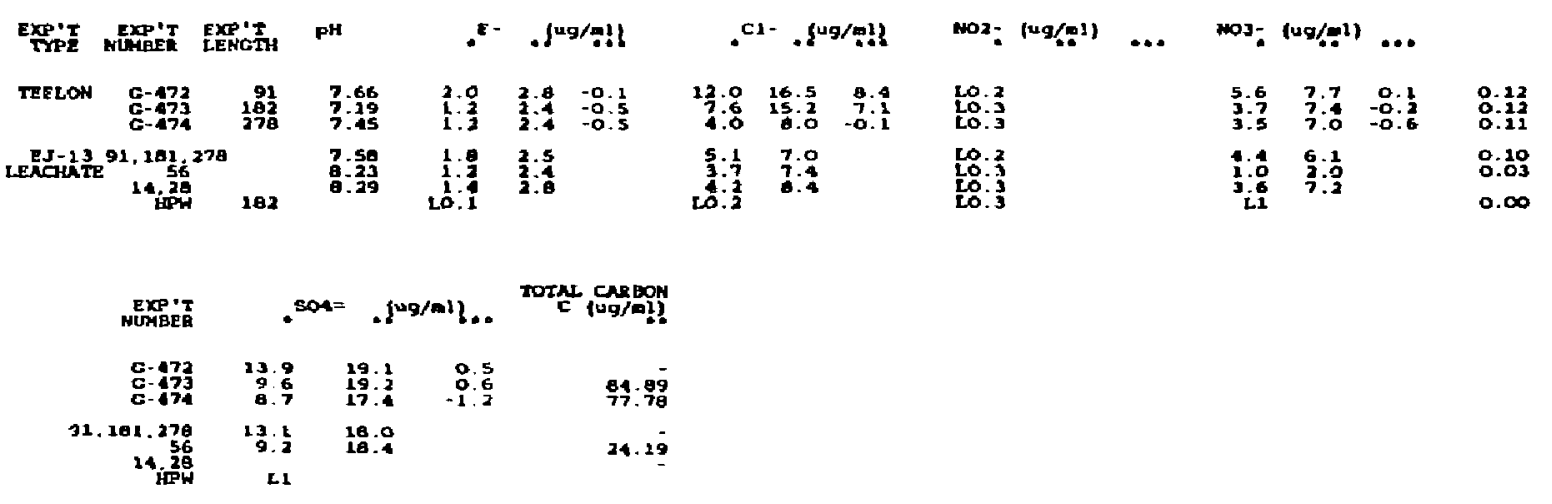

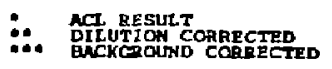


TABLE A-11

Anion Results from OR (Teflon Gasket) Experiments

This Table presents the anion results in ppm from the OR (Teflon gasket) experiments. $\mathrm{NO}_{2}-$ was $\langle 0.3 \mathrm{ppm}$ in all of these solutions. 
Table A-11

\begin{tabular}{|c|c|c|c|c|c|}
\hline \multirow[b]{2}{*}{ Test Type } & \multirow[b]{2}{*}{$\begin{array}{c}\text { Test } \\
\text { Number }\end{array}$} & \multicolumn{4}{|c|}{ Concentration (ppm) } \\
\hline & & $\mathrm{F}^{-}$ & $\mathrm{Cl}^{-}$ & $\mathrm{NO}_{3}=$ & $\mathrm{SO}_{4}=$ \\
\hline ATH-1C & $\begin{array}{l}G-483 \\
G-484 \\
G-485 \\
G-485 \\
G-487 \\
G-488 \\
G-489 \\
G-490 \\
G-491 \\
G-492 \\
G-493 \\
G-494 \\
G-495 \\
G-496\end{array}$ & $\begin{array}{l}2.6 \\
2.7 \\
2.7 \\
2.7 \\
2.7 \\
2.6 \\
2.8 \\
2.6\end{array}$ & $\begin{array}{l}7.7 \\
7.8 \\
7.5 \\
7.5 \\
8.9 \\
8.8 \\
9.0 \\
8.6\end{array}$ & $\begin{array}{l}8.7 \\
8.7 \\
8.3 \\
8.5 \\
9.0 \\
8.8 \\
9.2 \\
9.9\end{array}$ & $\begin{array}{l}22.0 \\
21.7 \\
21.3 \\
21.4 \\
23.0 \\
22.5 \\
25.1 \\
25.2\end{array}$ \\
\hline $\begin{array}{c}\text { ATM-1c } \\
+ \\
\text { Tuff }\end{array}$ & $\begin{array}{l}G-497 \\
G-498 \\
G-499 \\
G-500 \\
G-501 \\
G-502 \\
G-503 \\
G-504 \\
G-505 \\
G-506 \\
G-507 \\
G-50 B \\
G-509 \\
G-510\end{array}$ & $\begin{array}{l}2.7 \\
3.0 \\
2.8 \\
2.8 \\
2.9 \\
2.8 \\
2.9 \\
3.1\end{array}$ & $\begin{array}{r}8.5 \\
8.2 \\
7.6 \\
7.9 \\
19.5 \\
9.1 \\
9.0 \\
8.6\end{array}$ & $\begin{array}{l}9.2 \\
8.8 \\
8.3 \\
8.4 \\
8.8 \\
9.2 \\
9.9 \\
8.8\end{array}$ & $\begin{array}{l}23.8 \\
22.8 \\
21.3 \\
21.6 \\
22.9 \\
22.6 \\
25.2 \\
24.8\end{array}$ \\
\hline ATM-8 & $\begin{array}{l}G-511 \\
G-512 \\
G-513 \\
G-514 \\
G-515 \\
G-516 \\
G-517 \\
G=518\end{array}$ & $\begin{array}{l}2.7 \\
2.7 \\
2.8 \\
2.7 \\
2.8 \\
3.0 \\
3.1 \\
3.1\end{array}$ & $\begin{array}{l}7.8 \\
7.8 \\
7.7 \\
7.5 \\
8.8 \\
8.3 \\
9.8 \\
9.8\end{array}$ & $\begin{array}{l}8.7 \\
8.6 \\
8.5 \\
8.3 \\
9.0 \\
8.5 \\
9.6 \\
9.8\end{array}$ & $\begin{array}{l}21.6 \\
21.7 \\
21.4 \\
21.3 \\
22.0 \\
21.2 \\
25.4 \\
25.3\end{array}$ \\
\hline
\end{tabular}


Table A-11 (Cont'd)

\begin{tabular}{|c|c|c|c|c|c|}
\hline \multirow[b]{2}{*}{ Test Type } & \multirow[b]{2}{*}{$\begin{array}{c}\text { Test } \\
\text { Nunber }\end{array}$} & \multicolumn{4}{|c|}{ Concentration (ppm) } \\
\hline & & $F^{-}$ & $\mathrm{Cl}^{-}$ & $\mathrm{NO}_{3}^{-}$ & $\mathrm{SO}_{4}=$ \\
\hline & $\begin{array}{l}G-519 \\
G-520 \\
G-521 \\
G-522 \\
G-523 \\
G-524\end{array}$ & & & & \\
\hline $\begin{array}{c}\text { ATM-8 } \\
+ \\
\text { Tuff }\end{array}$ & $\begin{array}{l}G-525 \\
G-526 \\
G-527 \\
G-528 \\
G=529 \\
G-530 \\
G-531 \\
G-532 \\
G-533 \\
G-534 \\
G-535 \\
G-536 \\
G-537 \\
G-538\end{array}$ & $\begin{array}{l}3.1 \\
2.9 \\
2.9 \\
2.9 \\
2.9 \\
2.9 \\
3.4 \\
3.4\end{array}$ & $\begin{array}{r}8.8 \\
8.4 \\
7.0 \\
8.0 \\
8.9 \\
8.6 \\
10.5 \\
10.3\end{array}$ & $\begin{array}{r}9.6 \\
9.1 \\
8.7 \\
8.6 \\
8.8 \\
9.3 \\
9.8 \\
10.1\end{array}$ & $\begin{array}{l}24.5 \\
23.1 \\
21.8 \\
21.8 \\
21.7 \\
22.7 \\
26.1 \\
25.9\end{array}$ \\
\hline $\begin{array}{l}\text { Blanks } \\
\text { EJ-13 } \\
\text { Only }\end{array}$ & $\begin{array}{l}G-539 \\
G-540 \\
G-541 \\
G-542 \\
G-543 \\
G-544 \\
G-545 \\
G-546 \\
G-547 \\
G-548 \\
G-549 \\
G-550 \\
G-551 \\
G-552\end{array}$ & $\begin{array}{l}2.9 \\
3.6 \\
2.6 \\
2.7 \\
2.6 \\
2.6 \\
2.8 \\
2.8\end{array}$ & $\begin{array}{l}8.1 \\
8.6 \\
7.9 \\
8.1 \\
8.1 \\
8.1 \\
9.3 \\
8.9\end{array}$ & $\begin{array}{l}8.3 \\
8.9 \\
8.6 \\
8.7 \\
8.8 \\
8.5 \\
9.2 \\
9.4\end{array}$ & $\begin{array}{l}21.4 \\
22.1 \\
20.9 \\
20.9 \\
21.0 \\
21.0 \\
24.1 \\
23.9\end{array}$ \\
\hline
\end{tabular}

Cant'd 
Table A-11 (Cont'd)

\begin{tabular}{|c|c|c|c|c|c|}
\hline \multirow[b]{2}{*}{ Test Type } & \multirow[b]{2}{*}{$\begin{array}{l}\text { Test } \\
\text { Number }\end{array}$} & \multicolumn{4}{|c|}{ Concentration (ppm) } \\
\hline & & $F^{-}$ & $\mathrm{Cl}^{-}$ & $\mathrm{NO}_{3}-$ & $\mathrm{SO}_{4}=$ \\
\hline $\begin{array}{c}\text { Blanks } \\
+ \\
\text { Tuff }\end{array}$ & $\begin{array}{l}G-553 \\
G-554 \\
G-555 \\
G-556 \\
G-557 \\
G-558 \\
G-559 \\
G-560 \\
-561 \\
G-562 \\
G-563 \\
G-564 \\
G-565 \\
G-566\end{array}$ & $\begin{array}{l}2.8 \\
2.9 \\
2.9 \\
2.7 \\
2.7 \\
3.2 \\
3.0 \\
3.0\end{array}$ & $\begin{array}{l}8.5 \\
8.5 \\
8.5 \\
8.1 \\
8.8 \\
9.1 \\
9.5 \\
9.8\end{array}$ & $\begin{array}{l}8.9 \\
9.0 \\
9.2 \\
8.5 \\
8.7 \\
8.9 \\
9.3 \\
9.6\end{array}$ & $\begin{array}{l}21.5 \\
21.8 \\
22.3 \\
20.7 \\
21.3 \\
21.6 \\
24.4 \\
25.5\end{array}$ \\
\hline
\end{tabular}




\section{TAGLE A-12}

Np and Pu Release from ATM-8 Glass, 2R Experiments

$N p$ and Pu release is presented for the $2 R$ experiments. The cps columns are per second normalized to $0.1 \mathrm{~mL}$ for nonfiltered, filtered, and acid-soak solutions. The nonfiltered alfquot was taken upon opening the vessel. The filtered aliquot was obtained by filtering through $0.1 \mu \mathrm{m}$ filters for the 7-day solutions and through $50 \AA$ filters for the 28 - and 56-day samples. 
Tablo A-12. Np and Pu Release Troe ATM-B Ol ass

\begin{tabular}{|c|c|c|c|c|c|c|c|c|c|c|c|c|}
\hline \multirow{2}{*}{$\begin{array}{l}2^{237} \\
\text { Days }\end{array}$} & \multicolumn{6}{|c|}{ ATU-A Disks } & \multicolumn{6}{|c|}{ ATH-8 Diaks - Tuff } \\
\hline & pH & (Wonf iltered) & (Filtered) & (Acid Soak) & $\begin{array}{l}\text { Totel Np } \\
\text { Relasso } \\
\text { (oicro on ) }\end{array}$ & (ML) Mp & pH & (Menf ilitared) & (Filterod) & (Acid Soaks) & $\begin{array}{l}\text { Total Mp } \\
\text { Roleaso } \\
\text { (aicro os) }\end{array}$ & (ML) $\mathrm{Hp}_{\mathrm{p}}$ \\
\hline $\begin{array}{r}7 \\
7 \\
14 \\
14 \\
28 \\
28 \\
58 \\
58\end{array}$ & $\begin{array}{l}0.60 \\
7.60 \\
0.00 \\
7.18 \\
8.92 \\
7.01 \\
7.60 \\
7.37\end{array}$ & $\begin{array}{l}\text { 6. } . E-2 \\
6.6 E-2 \\
2.3 E-1 \\
2.3 E-1 \\
4.2 E-1 \\
4.1 E-1 \\
2.8 E-1 \\
3.5 E-1\end{array}$ & $\begin{array}{l}0.4 E-2 \\
2.0 \mathrm{E}-1 \\
1.9 \mathrm{E}-1\end{array}$ & $\begin{array}{l}\text { C. BE-2 } \\
\text { B.EE-2 } \\
\text { 3.1E-1 } \\
2.6 E-1 \\
\text { 6.JE-1 } \\
7.2 E-1 \\
\text { T.EE-1 } \\
\text { 2. GE-1 }\end{array}$ & $\begin{array}{c}1.67 \\
2.41 \\
0.41 \\
7.37 \\
17.8 \\
20.4 \\
11.6 \\
6.02\end{array}$ & $\begin{array}{r}0.8 \\
1.8 \\
4.6 \\
8.1 \\
10.8 \\
8.8 \\
4.1\end{array}$ & $\begin{array}{l}7.06 \\
7.02 \\
7.20 \\
7.23 \\
7.20 \\
7.09 \\
7.40 \\
7.01\end{array}$ & $\begin{array}{l}5.2 E-2 \\
5.1 E-2 \\
1.0 E-1 \\
2.0 E-1 \\
2.7 E-1 \\
1.1 E-1 \\
3.0 E-1 \\
2.0 E-1\end{array}$ & $\begin{array}{l}\text { 6.4E-2 } \\
3.1 E-1 \\
2.4 E-1\end{array}$ & $\begin{array}{l}2.2 E-2 \\
4.3 E-2 \\
1.6 E-1 \\
2.1 E-1 \\
3.0 E-1 \\
3.6 E-1 \\
3.0 E-1 \\
4.7 E-1\end{array}$ & $\begin{array}{r}1.70 \\
1.36 \\
1.02 \\
6.26 \\
11.3 \\
11.0 \\
11.2 \\
23.7\end{array}$ & $\begin{array}{l}.4 .1 \\
.7 \\
2.5 \\
3.2 \\
5.8 \\
5.8 \\
5.8 \\
0.9\end{array}$ \\
\hline \multicolumn{13}{|c|}{ 239Pu Aralysus } \\
\hline Days & PH & (Monfilterad) & (Filtered) & (Acid Soik) & $\begin{array}{l}\text { Total Pu } \\
\text { Relesso } \\
\text { (aicro on) }\end{array}$ & (N)pu & pH & (Wont iltered) & (Filtered) & (hcid Somk) & $\begin{array}{l}\text { Total Pu } \\
\text { Ruleaso } \\
\text { (iicro on) }\end{array}$ & (M) Pu \\
\hline $\begin{array}{l}7 \\
7 \\
34 \\
14 \\
28 \\
20 \\
66 \\
66\end{array}$ & $\begin{array}{l}6.08 \\
7.60 \\
8.80 \\
7.18 \\
0.92 \\
7.01 \\
7.60 \\
7.37\end{array}$ & $\begin{array}{l}4.8 E-2 \\
4.8 E-2 \\
3.1 \mathrm{EE-1} \\
1.8 \mathrm{EE-1} \\
2.4 \mathrm{EE-1} \\
2.3 \mathrm{BE}-1 \\
\text { 5. BE-2 } \\
\text { 5.3E-2 }\end{array}$ & $\begin{array}{l}4.1 E-2 \\
7,7 E-2 \\
5,3 E-2\end{array}$ & $\begin{array}{l}1.0 E-1 \\
1.3 \mathrm{EE-1} \\
7.7 \mathrm{E}-1 \\
6.9 \mathrm{E}-1 \\
1.62 \\
1.57 \\
1.67 \\
0.8 \mathrm{EE}-1\end{array}$ & $\begin{array}{r}91.9 \\
94.4 \\
235.4 \\
139.7 \\
543.6 \\
482.0 \\
518.1 \\
273.6\end{array}$ & $\begin{array}{l}0.04 \\
0.48 \\
0.46 \\
.93 \\
6.09 \\
1.04 \\
0.63\end{array}$ & $\begin{array}{l}7.05 \\
7.42 \\
7.20 \\
7.23 \\
7.20 \\
7.09 \\
7.48 \\
7.01\end{array}$ & $\begin{array}{l}1 . \Delta E-2 \\
1.4 E-2 \\
4.0 E-2 \\
3.8 E-2 \\
6.8 E-2 \\
7.3 E-2 \\
5.9 E-2 \\
4.4 E-2\end{array}$ & $\begin{array}{l}6.0 E-2 \\
4.6 E-2\end{array}$ & $\begin{array}{l}3.4 E-2 \\
6.0 E-2 \\
2.2 E-1 \\
2.0 E-1 \\
7.9 E-1 \\
7.0 E-1 \\
7.1 E-1 \\
8.0 E-1\end{array}$ & $\begin{array}{r}18.6 \\
10.8 \\
02.1 \\
79.3 \\
242.8 \\
210.4 \\
210.1 \\
211.0\end{array}$ & $\begin{array}{r}0.42 \\
6.12 \\
6.12 \\
6.48 \\
0.42 \\
6.43 \\
6.41\end{array}$ \\
\hline
\end{tabular}


Tabla A-12 (Cont'd

\begin{tabular}{|c|c|c|c|c|c|c|}
\hline \multicolumn{3}{|c|}{$237_{\mathrm{Hp}}$ Analyses } & \multicolumn{4}{|c|}{ ATU-8 Disks Crushed } \\
\hline Days & pH & (Nonfiltared) & (Filtared) & (Acid Soak) & $\begin{array}{l}\text { Total Hp } \\
\text { Asloses: } \\
\text { (wiero on) }\end{array}$ & $\left(H_{L}\right)_{H P_{p}}$ \\
\hline $\begin{array}{r}7 \\
7 \\
14 \\
14 \\
26 \\
26 \\
50 \\
60\end{array}$ & $\begin{array}{l}8.60 \\
8.85 \\
7.10 \\
7.04 \\
7.37 \\
7.42 \\
7.74 \\
7.86\end{array}$ & $\begin{array}{l}2.5 \mathrm{E}-2 \\
2.7 \mathrm{E}-2 \\
1.1 \mathrm{E}-1 \\
1.6 \mathrm{E}-1 \\
2.2 \mathrm{E}-1 \\
2.9 \mathrm{E}-1 \\
1.4 \mathrm{E}-1 \\
1.5 \mathrm{E}-1\end{array}$ & $\begin{array}{l}2.7 E-2 \\
1.1 E-1 \\
0.1 E-2\end{array}$ & $\begin{array}{l}1.4 \mathrm{E}-2 \\
4.2 \mathrm{E}-2 \\
1.3 \mathrm{E}-1 \\
1.6 \mathrm{E}-1 \\
1.7 \mathrm{E}-1 \\
3.7 \mathrm{E}-1 \\
\mathrm{MD} \\
\mathrm{MD}\end{array}$ & $\begin{array}{r}0.84 \\
1.21 \\
3.77 \\
12.38 \\
7.78 \\
3.98 \\
4.26\end{array}$ & $\begin{array}{r}8.1 \\
8.2 \\
8.8 \\
2.7 \\
1.3 \\
6.7\end{array}$ \\
\hline \multicolumn{7}{|c|}{ 239Py Analyses } \\
\hline Days & $\mathrm{pH}$ & (Honfiltored) & (Filitered) & (acid Sosk) & $\begin{array}{l}\text { Takob Pus } \\
\text { Rolonet } \\
\text { (Gicro go) }\end{array}$ & (ML) pu \\
\hline $\begin{array}{r}7 \\
7 \\
14 \\
14 \\
28 \\
28 \\
68 \\
66\end{array}$ & $\begin{array}{l}0.84 \\
6.95 \\
7.10 \\
7.04 \\
7.97 \\
7.42 \\
7.74 \\
7.08\end{array}$ & $\begin{array}{l}\text { 1.9E-2 } \\
6.2 \mathrm{E}-2 \\
1.6 \mathrm{E}-1 \\
1.7 \mathrm{E}-1 \\
1.1 \mathrm{E}-1 \\
1.2 \mathrm{E}-1 \\
7.9 \mathrm{E}-2 \\
\text { 8.1 }\end{array}$ & $\begin{array}{l}5.3 E-2 \\
6.3 E-2\end{array}$ & $\begin{array}{l}\text { 1. } 2 \mathrm{E}-1 \\
\text { 9.5E-2 } \\
3.5 \mathrm{E}-1 \\
3.1 \mathrm{E}-1 \\
7.5 \mathrm{E}-1 \\
9.0 \mathrm{E}-1 \\
1.62 \\
1.70\end{array}$ & $\begin{array}{r}34.1 \\
34.7 \\
97.2 \\
89.7 \\
221.4 \\
272.2 \\
409.4 \\
634.1\end{array}$ & 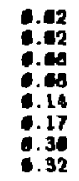 \\
\hline
\end{tabular}


TABLE A-13

FY 1986 Actinides

This Table presents the Np and Pu results for the $1 R$ experiments. The Table is annotated at the bottom of the Table. 
Table A-13. FY 1986 Actinides

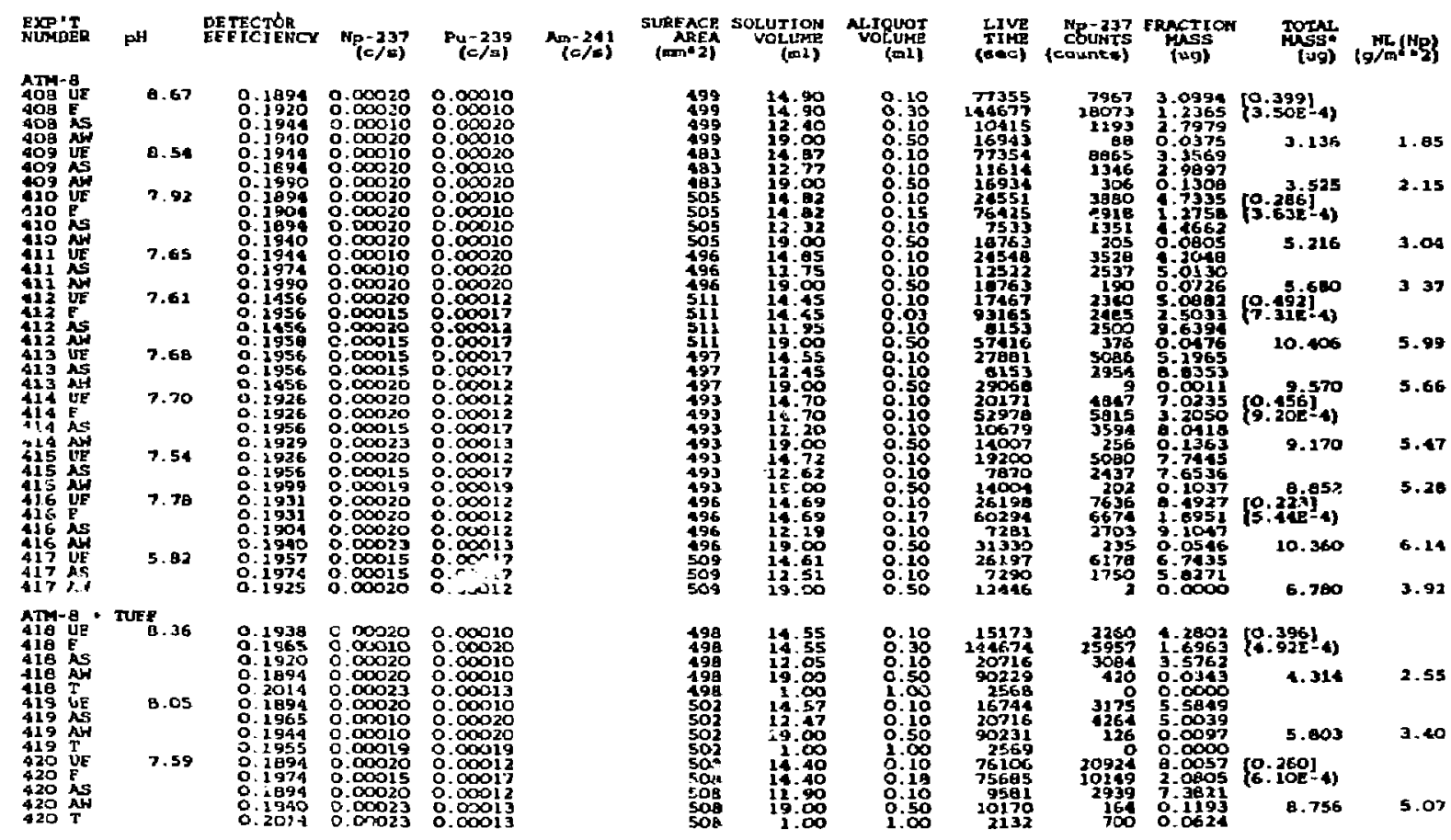


Table A-13 (Cont d)

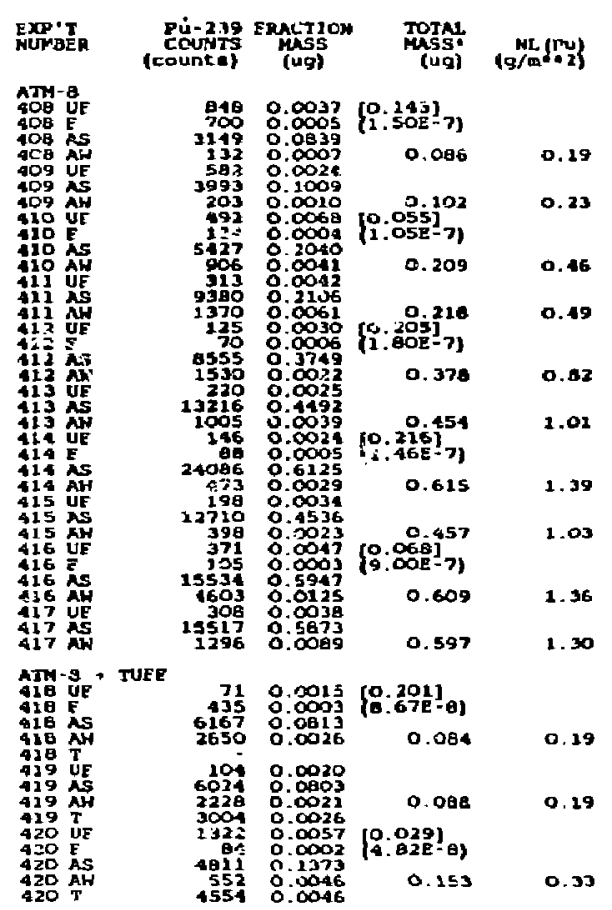


Table A-13 (Cont'd)
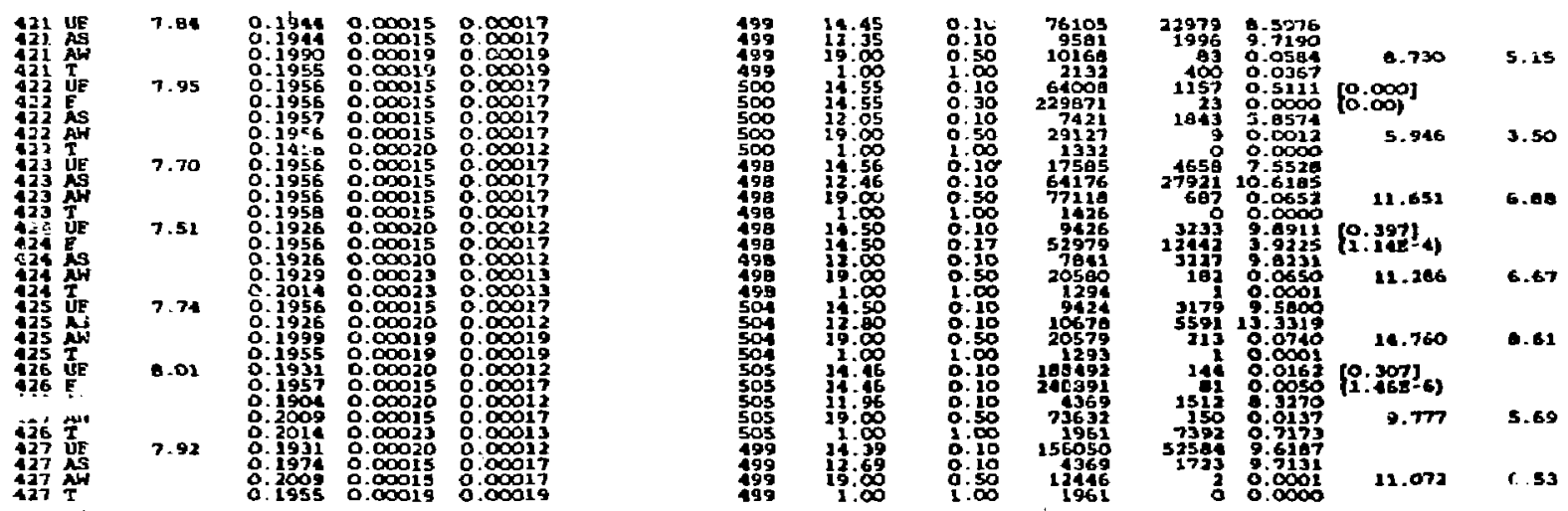

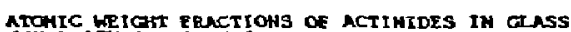

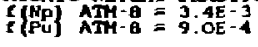

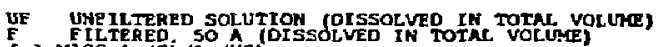

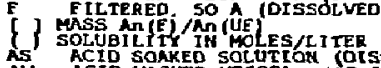

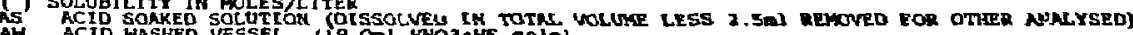

T TUFE WAFER SUREACE (VALUE REPOATEO REFEkS TO ONLY OHE SIDE OE WAFER)

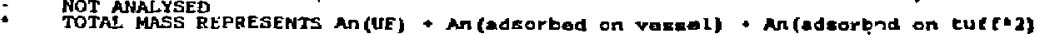


Table A-13 (Cont'd)

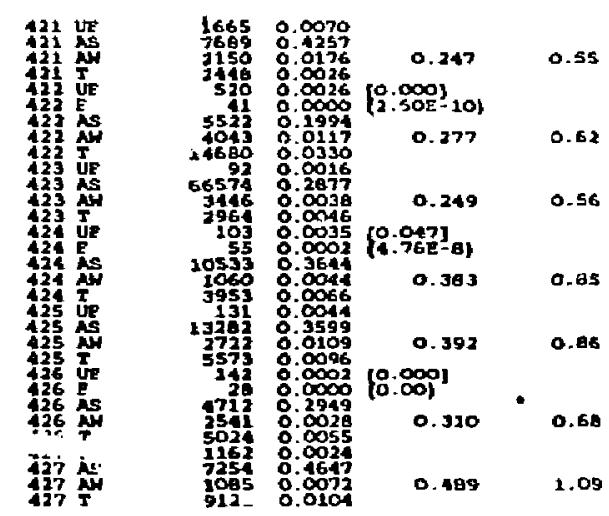


TABLE A-14

Np and Pu Results from the OR (Teflon Gasket) Experinients

This Table presents Np and Pu data from the OR (Teflon gasket) experiments. The columns are:

Effey = detector efficiency

$L T=$ Iive counting time (sec)

Np, Pu, $A m=$ background levels for these elements, Am is not used aliquot = the volume of solution courited sol'n = volume of the solution from which the counted aliquot was extracted

counts = raw counts for each element

$c / s=$ counts per second

$B G C=$ background corrected counts/sec

$E C=$ efficiency corrected disintegrations/sec

Total $d / s=$ total disintegrations for each element

Total $\mu \mathrm{gm}=$ total micrograms for each element

The normalized mass loss for each experiment is given in the unmarked columr. 
Table A-14. Np and Pu Results from the OR (Teflon Gasket) Experiments

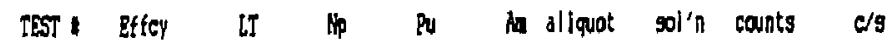
14 day

\begin{tabular}{|c|c|c|c|c|c|c|c|c|c|}
\hline $5 ! 1 *$ & .1938 & 91441 & .00022 & .00013 & .0011 & .10 & 14.87 & 1379 & .015 \\
\hline $511 \times$ & .1936 & 85555 & .00023 & .00013 & .0011 & .15 & 14.87 & 743 & \\
\hline $511 \mathrm{r}$ & .2030 & 59773 & .00019 & .00019 & .0004 & .10 & 19.89 & 1005 & \\
\hline $5 ! 1+4$ & .1938 & 104113 & .00023 & .00013 & .0011 & .50 & 19.00 & 60 & \\
\hline $2 \pi$ & .2030 & 91431 & .00019 & 00019 & .0004 & .10 & 14.90 & 1601 & \\
\hline $2 n$ & .2030 & 23557 & .00019 & .00019 & .0604 & .10 & 12.39 & 485 & \\
\hline $12 \mathrm{nhH}$ & .2030 & 104121 & .00019 & 00019 & .0004 & .50 & 19.00 & 60 & \\
\hline 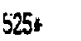 & .1938 & 59778 & .00023 & .00013 & .0011 & 10 & 14,19 & 2176 & \\
\hline $525+4$ & .2030 & 85555 & .00019 & .00019 & .0004 & .30 & 14.49 & 3926 & \\
\hline $525+4$ & 1938 & 99007 & .00023 & .00013 & .0011 & .10 & 11.40 & $415 !$ & \\
\hline 525 tat & .1938 & 245057 & .00023 & .00013 & .0011 & .50 & 19.00 & 87 & \\
\hline $525 \mathrm{TUPP}$ & .2597 & 10651 & .00023 & .00013 & .0011 & 1.00 & 1.00 & 3 & \\
\hline 64 & .1938 & 80418 & .00023 & .00013 & .001 & .10 & 14.48 & 2720 & \\
\hline $526: 00$ & .2030 & 00455 & .00019 & .00019 & .0004 & .10 & 11.86 & 3192 & \\
\hline $526+44$ & .2030 & $2450 ! 0$ & .00019 & .00019 & .0004 & .50 & 19.00 & 60 & \\
\hline 52TVPP & .2720 & 10651 & .00019 & .00019 & .0004 & 1.000 & 1.00 & 3 & \\
\hline
\end{tabular}

28 QAP

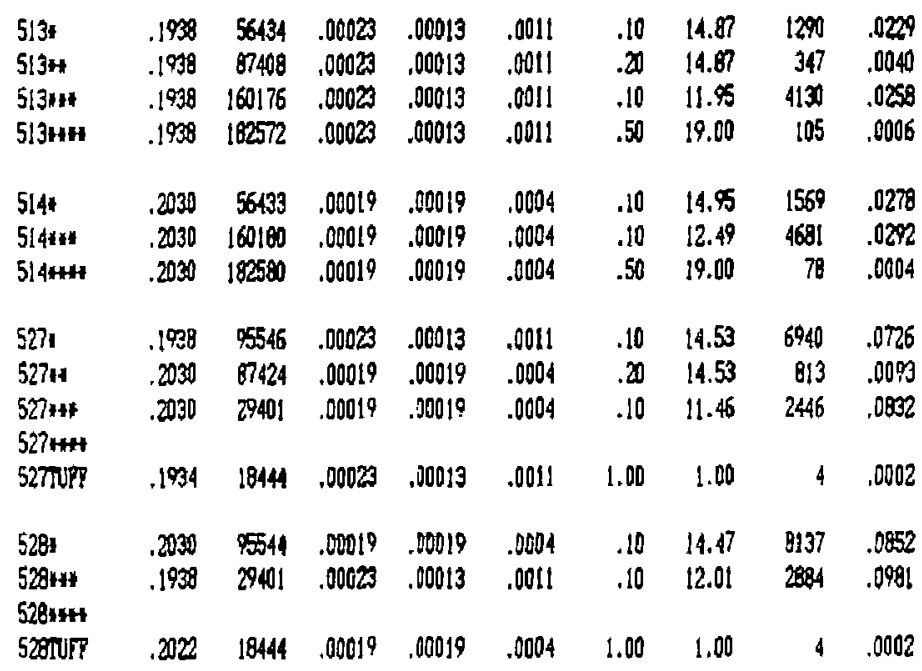


Table A-14 (Cont'd)

\begin{tabular}{|c|c|c|c|c|}
\hline $\begin{array}{l}\text { TRT } 1 \\
14 \text { dAY }\end{array}$ & $B C$ & $x$ & $\begin{array}{r}\text { total } \\
d / 3\end{array}$ & 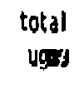 \\
\hline S!IF & $.0149^{\circ}$ & .7669 & 11.39 & (488). \\
\hline stlu & .0085 & .2009 & 4.32 & .11860 \\
\hline 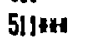 & .0166 & .8189 & 9.73 & .3742 \\
\hline 511w+4 & .0003 & .0036 & .07 & $\begin{array}{l}.0026 \\
.4110\end{array}$ \\
\hline $512 \pi$ & .0173 & .8532 & 12.71 & . 4890 \\
\hline $5\{2+14$ & .0201 & .9879 & 12.24 & .4708 \\
\hline $51221+4$ & .0004 & .0038 & .07 & $\begin{array}{r}.0028 \\
.090\end{array}$ \\
\hline 525 & .0362 & 1.8664 & 27.04 & 1.0402 \\
\hline $52+1$ & .0457 & .7504 & 10.87 & .4182 \\
\hline $525 \pm 11$ & .0414 & 2.1342 & 24.33 & .9359 \\
\hline $525+1+4$ & .0001 & .0013 & .02 & .0009 \\
\hline $525 \mathrm{~N} / \mathrm{m}$ & .0001 & .0002 & .00 & $\begin{array}{r}.0000 \\
1.0410\end{array}$ \\
\hline 34 & .0336 & 1.7734 & 25.10 & .9654 \\
\hline $5261+1$ & .0395 & 1.9460 & 23.08 & .887 \\
\hline $526+1914$ & .0001 & .0005 & .01 & .0004 \\
\hline 525TU?? & .0001 & .0003 & .00 & .0000 \\
\hline
\end{tabular}

28 diY

\begin{tabular}{|c|c|c|c|c|c|}
\hline 5131 & .0226 & 1.1676 & 17.36 & .6678 & \\
\hline $513 *$ & .0037 & .0965 & 1.13 & .0552 & \\
\hline 5134: & .0856 & 1.3186 & 15.76 & .6060 & \\
\hline \multirow[t]{2}{*}{$513 \mathrm{HAn}$} & .0003 & 0036 & .07 & .0026 & \\
\hline & & & & .6710 & .4 \\
\hline 51414 & .0276 & $\begin{array}{l}1.3602 \\
1\end{array}$ & 20.34 & . 7821 & \\
\hline \multirow[t]{2}{*}{$514 \mathrm{HH}$} & .0002 & .0023 & .04 & .0017 & \\
\hline & & & & .7840 & .4 \\
\hline 5271 & .0724 & 3.7361 & 54.24 & 2.0879 & \\
\hline $527 \mathrm{u}$ & .0099 & .244 & 3.26 & .1254 & \\
\hline $\begin{array}{l}5271+4 \\
527+4+\end{array}$ & .0830 & 4.0889 & 46.86 & 1.8023 & \\
\hline $527 \mathrm{nPp}$ & .0000 & -.0001 & .00 & -.0000 & $x$ \\
\hline 5281 & P. & 4.1860 & 60.57 & $2.32 \%$ & \\
\hline 52314 & .0979 & $5.09 \%$ & 60.65 & 2.3325 & \\
\hline 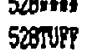 & .8000 & mang & ת & & \\
\hline & & & & 2.3500 & 3 \\
\hline
\end{tabular}


Table A-14 (Cont'd)

56 a.4

\begin{tabular}{|c|c|c|c|c|c|c|c|c|}
\hline 157 & 1999 & 30599 & .00021 & .00915 & .0011 & .10 & 14.90 & 1733 \\
\hline 154 & .1009 & 82007 & .00021 & .00015 & .0011 & .40 & 14.97 & 8120 \\
\hline fFI & 19099 & 54230 & .00021 & .00015 & ,001 & .10 & 11.85 & 3435 \\
\hline 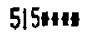 & .1934 & 55608 & .00021 & .00015 & .001 & .50 & 19. & (4) \\
\hline & 973 & 30599 & .00019 & .00019 & .000 & .10 & 14.87 & 1538 \\
\hline & 73 & 32 & .00019 & .000019 & .0004 & .10 & 12 & 5573 \\
\hline jort & .2020 & 55610 & 00019 & .00019 & .0000 & 50 & 19.00 & 62 \\
\hline & 909 & 1050173 & .00821 & .00015 & .001 & .10 & 14. & 85577 \\
\hline 941 & .1973 & 87008 & .00019 & .00019 & .0004 & 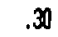 & 14.47 & 5499 \\
\hline $\mathrm{HH}$ & .1909 & 15626 & .00021 & .00015 & .0011 & .10 & 11.86 & 1301 \\
\hline HH & .1931 & 20202 & .00021 & .00015 & .0011 & .50 & 19.00 & $\mathfrak{1}$ \\
\hline TUPF & .1993 & 5627 & .00021 & .00015 & .0011 & 1.00 & 1.00 & 2 \\
\hline & 973 & 105059 & .00019 & .00019 & .000 & .1 & & 8319 \\
\hline HH & .1973 & 13625 & .00019 & .00019 & .0004 & .10 & 11.98 & 1173 \\
\hline+4 & .2022 & 291 & .08019 & .0001 & .0004 & .50 & 19,00 & 75 \\
\hline & .2022 & 5827 & 0019 & 19 & .0004 & 1.00 & 1.00 & 9 \\
\hline
\end{tabular}

91 DAP

\begin{tabular}{|c|c|c|c|c|c|c|c|c|c|}
\hline & .1934 & 18817 & .00022 & .00013 & .0011 & .20 & 14.91 & 1791 & .0978 \\
\hline $17 \mathbf{H}$ & .1934 & 553572 & .00022 & .00013 & .0011 & .35 & 14,91 & 4490 & .0088 \\
\hline $17 \mathrm{HH}$ & 1934 & 52800 & .00022 & .00013 & .0011 & .20 & 11.62 & 5500 & .1231 \\
\hline $5171+4$ & 1094 & 103124 & .00022 & .00013 & .0011 & .50 & 19.00 & 50 & .0005 \\
\hline 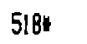 & .2022 & 18315 & .00019 & .00019 & .0004 & .20 & 14,91 & 1892 & .1033 \\
\hline $8 \mathbf{H H}$ & .20122 & 52818 & .00019 & .00019 & .0004 & .20 & 12.21 & 6500 & .1231 \\
\hline HAH & .202 & 103124 & .00019 & .00019 & .0004. & .50 & 19,00 & 50 & .0005 \\
\hline $59 ! 14$ & .1994. & 17156 & , 00022 & .00013 & .0011 & .20 & 14,50 & 2770 & .1655 \\
\hline 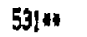 & .2022 & 55571 & .00019 & .00019 & .0004 & .40 & 14.50 & 9602 & .1728 \\
\hline HH & 1994 & 6531 & .00022 & .00013 & .0011 & .20 & 11.19 & 1200 & .1820 \\
\hline $\mathbf{m + n}$ & .1934. & $15 \pi 019$ & .00022 & .00013 & .0011 & .50 & 19.00 & 300 & .0019 \\
\hline TUFT & . 1934. & 7 & .00022 & .00013 & .0011 & 1.00 & 1.00 & 2 & .0003 \\
\hline & .2022 & 17156 & .00019 & .00019 & .0004 & .20 & 14.52 & 2863 & .1669 \\
\hline & $2 n$ & 6531 & .00019 & .80019 & .0004. & .20 & 11.70 & 12000 & .1807 \\
\hline$H$ & .2022 & 157502 & .00019 & .00019 & .0004 & .50 & 19,00 & 300 & .0019 \\
\hline ZTUPP & .2012 & 7231 & 00019 & .00319 & .0004 & 1.08 & 1.00 & 2 & .0003. \\
\hline
\end{tabular}


Tabie A-14 (Cont'd)

$56 \mathrm{DH}$

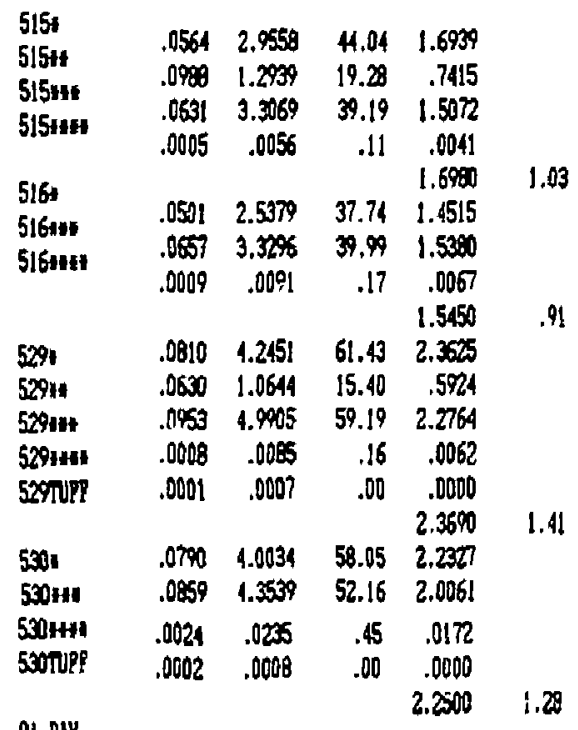

9) DAY

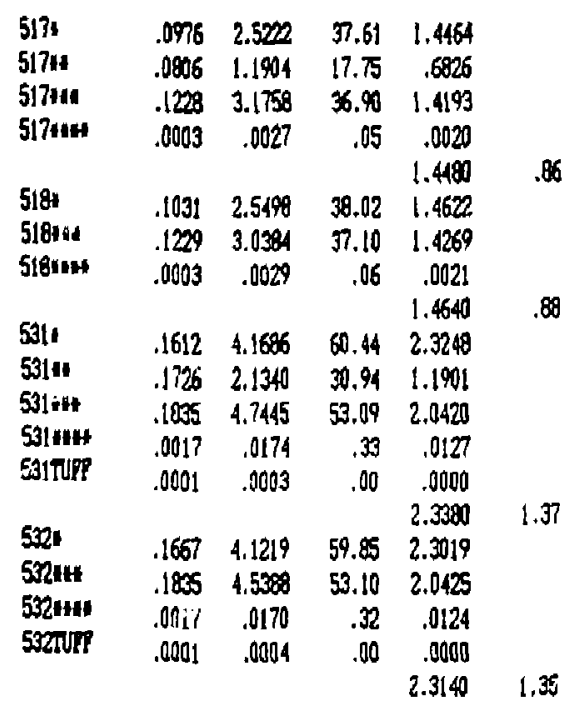


Table A-14 (wont'd)

$182 \mathrm{MY}$

\begin{tabular}{|c|c|c|c|c|c|c|c|c|c|}
\hline 5191 & .1907 & $7479 !$ & .00023 & .00013 & .0011 &. & 14.90 & 3178 & .0425 \\
\hline $519 k$ & .1971 & {$[4] 845$} & .00019 & .00019 & .0004 & .35 & 14.90 & 12137 & .0856 \\
\hline $519 \mathrm{AH}$ & .1971 & 8019 & .00019 & .00019 & .0004 & .1 & 11.65 & 500 & .0601 \\
\hline $5194 m$ & .1943 & 15617 & .00023 & .00013 & .0011 & .5 & 19.00 & 4 & 10003 \\
\hline $520 *$ & $|97|$ & 74791 & .00019 & .00019 & .0084 & .1 & 14.87 & 3597 & .0481 \\
\hline 52014 & .1907 & 9786 & .00023 & .00013 & .0011 & .1 & 12.35 & 50 & .0562 \\
\hline $520 x+4$ & .1998 & 15617 & .00019 & .09019 & .0004 & .5 & 19.00 & 4 & .0003 \\
\hline 5397 & .1907 & 93218 & .00023 & .00013 & .0011 & .1 & 14.50 & 7634 & .0819 \\
\hline 5334 & .1907 & 5567 & .00023 & .00013 & .0011 & .25 & 14.50 & 4480 & .0805 \\
\hline $5331 \%$ & .1907 & 12031 & .00022 & $.000 \div 3$ & .0011 & .1 & 11.3 & 1270 & .1055 \\
\hline 52331EH & .1943 & 66139 & .00023 & .80013 & .0011 & .5 & 19.00 & 50 & .0008 \\
\hline $533 \pi / \mathrm{Fe}$ & & & & & & & & & IRRR \\
\hline 53411 & $197 !$ & 65466 & .00019 & .00019 & .0004 & .1 & 14.51 & 6031 & .0921 \\
\hline $534 \mathrm{ran}$ & .1971 & 120130 & .00019 & .00019 & .0004 & .1 & 12.01 & 1350 & .1122 \\
\hline 534 161: & .1998 & 66140 & .00019 & .00019 & .0004 & .5 & 19.00 & 25 & .0004 \\
\hline 534TUFP & & & & & & & & & $R O R$ \\
\hline
\end{tabular}

TEST Counts c/s BEC $\quad x$ total totai counts $\quad / s$ 14 day

P

\begin{tabular}{|c|c|c|c|c|c|c|c|c|c|}
\hline 5114 & 104 & .0011 & .0010 & .05200 &.$\pi$ & .00034 & & 114 & .00 \\
\hline 5114 & 45 & .0005 & .0004 & .0136 & .20 & .00009 & & 8 & .0010 \\
\hline $511+4$ & 1539 & $.025 ?$ & .0256 & 1.250 & 14.86 & .00659 & & 126 & .002 \\
\hline 511 HA & 86 & .0008 & .00007 & .0072 & .14 & $\begin{array}{l}.000055 \\
.00665\end{array}$ & .02 & 114 & .00 \\
\hline 5124 & 108 & .0012 & .0010 & .0488 & .73 & .00032 & & 46 & .0 \\
\hline $512+4 t$ & 668 & .0279 & .027 & 1.3512 & 16.90 & .00745 & & 65 & .00 \\
\hline $512+\cdots *$ & 83 & .0008 & .0006 & . 00600 & .11 & $\begin{array}{r}.00005 \\
.00075\end{array}$ & .02 & 33 & \\
\hline 5254 & 107 & .0018 & $.001 \%$ & .0857 & 1.24 & .00055 & & 66 & .0 \\
\hline $525+1$ & 240 & (1028 & .0026 & .0429 & .62 & .00027 & & 33 & .0 \\
\hline $525 * 4$ & 4234 & .0424 & .0423 & 2.1822 & 24.80 & $.010 \%$ & & 379 & .0 \\
\hline 525nent & 191 & .0008 & .0006 & .0067 & .13 & .50086 & & 256 & .90 \\
\hline $5251 \mathrm{I}$ P? & 2148 & .2017 & .2015 & $.776 !$ & .78 & $\begin{array}{r}.00034 \\
.0117\end{array}$ & .09 & 89 & \\
\hline $526+$ & 1211 & .0015 & .0014 & .0703 & 1.02 & .00045 & & $9 !$ & .00 \\
\hline $526+\#$ & 3684 & .0463 & .0481 & 2.359 & 28.11 & .01238 & & 306 & .06 \\
\hline $526+14$ & 76 & .0003 & .0001 & .0012 & .02 & .00001 & & 97 & .00 \\
\hline S26TUP? & 1729 & .1623 & .1621 & $.5 \% 1$ & .60 & $\begin{array}{l}.000026 \\
.01291\end{array}$ & .03 & 67 & .0 \\
\hline
\end{tabular}


Table A-14 (Cont'd)

182 AY

\begin{tabular}{|c|c|c|c|c|}
\hline $519 \%$ & .0420 & $2.216 !$ & 33.02 & 1.2700 \\
\hline 51911 & .0858 & 1.2276 & 18.44 & $.70 n$ \\
\hline 519116 & .0509 & 3.0397 & 36.02 & 1.3251 \\
\hline $519+11$ & .0000 & 0003 & .01 & $\begin{array}{r}.0002 \\
1.3065\end{array}$ \\
\hline 5001 & .0479 & 2.4004 & 36.14 & 1.3900 \\
\hline 50014 & .0560 & $2.985 \mathrm{I}$ & 3.25 & 1.3942 \\
\hline $5201+14$ & .0001 & .0007 & .01 & $\begin{array}{l}.0005 \\
\therefore .39417\end{array}$ \\
\hline 5331 & .0817 & 4.2620 & 62.09 & 2.3088 \\
\hline $533 \%$ & .0002 & 1.6829 & 24.40 & .936 \\
\hline $530 \mathrm{~m}$ & .1053 & 5.5200 & 62.40 & 2.3949 \\
\hline Sxon & .0005 & .0054 & .10 & .0010 \\
\hline $530 \pi / P$ & ERRI & ERTR & ERTR & errar \\
\hline 534 & .0919 & 1.8613 & 67.68 & 2.6031 \\
\hline 539111 & .1120 & 5.6825 & 68.25 & 2.6249 \\
\hline $5341+11$ & .0002 & .0019 & .04 & . 0014 \\
\hline $534 \pi$ JP? & DROR & MRAR & ERT & ERPRP \\
\hline
\end{tabular}

\begin{tabular}{|c|c|c|c|c|}
\hline $\begin{array}{l}\text { TRS I } \\
\text { It dap }\end{array}$ & $B C C$ & $x$ & $\begin{array}{r}\text { lotal } \\
\mathrm{d} / \mathrm{s}\end{array}$ & $\begin{array}{c}\text { total } \\
\text { nges }\end{array}$ \\
\hline $5 ! 14$ & 0.0000 & 0.0000 & 0.00 & 0.000 \\
\hline & 0.0000 & 0.0000 & 0.00 & 0.0 \\
\hline & 0.0000 & 0.0030 & 0.00 & .00 \\
\hline Sllнस & .0000. & 0.0000 & 0.00 & \\
\hline $512 \pi$ & 0.0000 & 0.0000 & 0.00 & 006 \\
\hline IIII & 0.0000 & 0.0000 & 0.00 & 0.00 \\
\hline $2 \mathrm{en}$ & 0.0000 & 0.0000 & 0.00 & 0.0 \\
\hline 5251 & 1.0000 & 0.0030 & 0.00 & 1.00 \\
\hline 525.1 & 0.0000 & 0.0000 & 0.00 & 0.0 \\
\hline $525+11$ & 0.0000 & 0.0000 & 0.00 & 0.00 \\
\hline 55 in: & 0.0000 & 0.00000 & 0.00 & 0.00 \\
\hline $525 T$ TPP & 0.0000 & 0.0000 & 0.00 & 0.08 \\
\hline & 0000 & 0.0000 & .00 & 0.000 \\
\hline & 0000 & 0.0000 & 0.00 & 0.080 \\
\hline 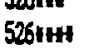 & 0.0000 & 0.0000 & 0.00 & 0.000 \\
\hline STIP? & 0.0000 & 0.00003 & 0.00 & \\
\hline
\end{tabular}


Table A-14 (Cont'd)

28 OAY

\begin{tabular}{|c|c|c|c|c|c|c|c|c|c|}
\hline $5\lfloor 31$ & 117 & .0021 & .0019 & .1003 & 1.49 & .00066 & & $\$ 1$ & .0009 \\
\hline |l & 29 & .0003 & .0002 & .0052 & .08 & .00803 & & 83 & .00 \\
\hline $513 \mathrm{nH}$ & 6281 & .0392 & .0391 & 2.0167 & 24.10 & .01062 & & 599 & 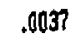 \\
\hline 513Hस & 233 & .0013 & .0011 & .0118 & .22 & $\begin{array}{l}.00010 \\
.01072\end{array}$ & .09 & 213 & , \\
\hline 5141 & 98 & $.001 ?$ & .0015 & .0762 & 1.14 & .00850 & & $2 \pi$ & \\
\hline $5 ! 4+4$ & $\$ 19$ & .0601 & $.05 \%$ & 2.9460 & 3.83 & .01623 & & 750 & \\
\hline $5 / 44+H$ & 265 & .0015 & .0013 & .0124 & .24 & $\begin{array}{l}.00010 \\
.01633\end{array}$ & .04 & $\infty$ & \\
\hline 778 & 146 & .0015 & .0014 & .0721 & 1.05 & 00046 & & 129 & \\
\hline $527 \times 1$ & 23 & .0003 & .0001 & .0018 & .03 & .00001 & & 30 & \\
\hline $\begin{array}{l}527+44 \\
527 \text { us }\end{array}$ & 3983 & .1355 & .1353 & 6.6641 & 76.37 & .03364 & & 266 & \\
\hline $52 \pi \mathrm{NT}$ & 7022 & .3007 & .3006 & 1.9679 & 1.97 & $\begin{array}{l}.090907 \\
.03559\end{array}$ & .09 & 235 & \\
\hline $528 I$ & 27 & .0024 & .0022 & .807 & 1.56 & .00069 & & 46 & \\
\hline $\begin{array}{l}528 \times 14 \\
528 \times+4 !\end{array}$ & 4112 & .1399 & .1397 & 7.2100 & 86.59 & .03815 & & 281 & \\
\hline gru?? & 12976 & .7035 & .7033 & 3.4785 & 3.48 & $\begin{array}{l}.00153 \\
.04121\end{array}$ & .10 & 460 & \\
\hline
\end{tabular}

56 Q4Y

\begin{tabular}{|c|c|c|c|c|c|c|c|c|c|}
\hline \$ISः & 68 & .0022 & .0021 & .1086 & 1.52 & .00071 & & 34 & .0011 \\
\hline $5 ! 5 \mathrm{HH}$ & 228 & .0028 & .0026 & .0344 & .51 & .00023 & & 108 & .0013 \\
\hline $515+4$ & $994 ?$ & .1834 & .1835 & 9.5999 & 119.76 & .05011 & & 681 & . 01 \\
\hline $515 \mathrm{n}+\mathrm{a}$ & 30 & .0005 & .0004 & .0840 & .09 & .00003 & 12 & 44 & .00 \\
\hline $5 ! 6 t$ & 67 & .0022 & .0020 & .1013 & 1.51 & .00056 & & 17 & \\
\hline $516+H$ & $793 !$ & .1462 & $.146 !$ & 7.4025 & 88.90 & .03916 & & 601 & .011 \\
\hline $5164+4$ & 113 & .0020 & .0018 & .0162 & .35 & $\begin{array}{l}.00615 \\
.03931\end{array}$ & .09 & 28 & \\
\hline 529. & 326 & $.003 !$ & .0030 & .1547 & 2.24 & .00099 & & 139 & \\
\hline $520 \mathrm{H}$ & 138 & .0016 & .0014 & .02236 & .34 & .00015 & & 46 & 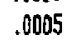 \\
\hline $5294 *$ & 3697 & .2713 & .2712 & 14.2048 & 168.47 & .07422 & & 226 & .01 \\
\hline $529 t+1$ & 40 & .0014 & .0012 & .0126 & .24 & .00011 & & 32 & .00 \\
\hline $529 T \mathrm{JP} P$ & 5516 & .9466 & .9465 & 4.8939 & 4.89 & $\begin{array}{l}.00216 \\
.07065\end{array}$ & .18 & 189 & .03 \\
\hline 530: & 278 & .0026 & .0025 & .1245 & 1.80 & .00020 & & 51 & .00 \\
\hline $520 *+1$ & 3490 & $.256 !$ & .2560 & 12.9730 & 155.42 & .06847 & & 243 & \\
\hline 500แ: & 140 & .0048 & .0046 & .0456 & .87 & .00038 & & 32 & \\
\hline DTUP? & 5259 & .9025 & .9020 & 1.4625 & 4.46 & .00197 & 15 & 179 & \\
\hline
\end{tabular}


Table A-14 (Cont'd)

$28 \mathrm{MP}$

$\begin{array}{llllll}5131 & 0.0000 & 0.0000 & 0.00 & 0.000\end{array}$

$\begin{array}{lllll}513.1 & 0.0000 & 0.0000 & 0.00 & 0.000\end{array}$

S13un $\quad 0.0000 \quad 0.0000 \quad 0.00 \quad 0.000$

$\begin{array}{lllll}513 \mathrm{HH} & 0.0000 & 0.0000 & 0.00 & 0.000\end{array}$

$\begin{array}{lllll}514 k & 0.0000 & 0.0000 & 0.00 & 0.000\end{array}$

$\begin{array}{lllll}514 \mathrm{HH} & 0.0000 & 0.0000 & 0.00 & 0.000\end{array}$

$\begin{array}{lllll}51 \text { Litw } & 0.0000 & 0.0000 & 0.00 & 0.000\end{array}$

$\begin{array}{lllll}527 \mathrm{r} & 0.0000 & 0.0000 & 0.00 & 0.000\end{array}$

$\begin{array}{lllll}527 \mathrm{n} & 0.0000 & 0.0000 & 0.00 & 0.000\end{array}$

$\begin{array}{lllll}527+11 & 0.0000 & 0.0000 & 0.00 & 0.000\end{array}$

527 tan

$\begin{array}{lllll}527 n P^{2} & 0.0000 & 0.0000 & 0.00 & 0.000\end{array}$

$\begin{array}{lllll}5281 & 0.0000 & 0.0000 & 0.00 & 0.000\end{array}$

$\begin{array}{lllll}52814 \pi & 0.0000 & 0.0000 & 0.00 & 0.000\end{array}$

5x81+4

5207up? $\quad 0.0000 \quad 0.0000 \quad 0.00 \quad 0.000$

56 גXY

\begin{tabular}{|c|c|c|c|c|}
\hline 515x & 0.0000 & 0.0000 & 0.00 & 0.000 \\
\hline 5154 & 0.0000 & 0.00000 & 0.00 & 0.000 \\
\hline 515H & 0.0000 & 0.0000 & 0.00 & 0.000 \\
\hline $515 \mathrm{HAH}$ & 0.0000 & 0.00000 & 0.00 & 0.000 \\
\hline 5164 & 0.0000 & 0.00000 & 0.00 & 0.080 \\
\hline 51654 & 0.0000 & 0.0000 & 0.00 & 0.000 \\
\hline $5164 n$ & 0.0000 & 0.0000 & 0.00 & 0.000 \\
\hline 529 & 0.0000 & 0,0000 & 0.00 & 0.000 \\
\hline 529.1 & 0.0000 & 0.00000 & 0,00 & 0.000 \\
\hline 529111 & 0.0000 & 0.0000 & 0.00 & 0.000 \\
\hline $5 \times 4+4$ & 0.00300 & 0.0600 & 0.80 & 0.080 \\
\hline $5 x+4 \mathrm{dith}$ & 0.0000 & 0.0000 & 0.00 & 0.000 \\
\hline 5301 & 0.0000 & 0.0000 & 0.00 & 0.000 \\
\hline 53 & 0,0000 & 0.0000 & 0.00 & 0.000 \\
\hline ont & & & & \\
\hline
\end{tabular}


Tisie A-14 (Cont'd)

Q1 QRY

\begin{tabular}{|c|c|c|c|c|c|c|c|}
\hline $517 *$ & $19 ?$ & .0108 & .0106 & .2747 & 4.10 & $.0812 x$ & \\
\hline $517 k$ & 81 & .0015 & .013 & .019\% & .29 & .00013 & \\
\hline $517 \mathrm{kH}$ & 38541 & .7316 & .7315 & 18.9112 & 219.75 & $.0988 !$ & \\
\hline 517 u4 & 1853 & .0180 & .0176 & .1845 & 3.51 & $\begin{array}{l}.00154 \\
.098255\end{array}$ & .23 \\
\hline $51^{9}$ & 90 & .0049 & $.004 ?$ & .1168 & 1.74 & .0007 & \\
\hline 51844 & $36 ! 5 !$ & .6844 & .6843 & 16.9002 & 206.60 & .09101 & \\
\hline $518 H+4$ & 920 & .0089 & .0087 & .0864 & 1.64 & $\begin{array}{l}.000072 \\
.09173\end{array}$ & .22 \\
\hline $531 \%$ & 156 & .0091 & .0099 & .2317 & 3.36 & .00148 & \\
\hline $53 ! 14$ & 213 & .0041 & .0042 & $.051 \hat{\imath}$ & .75 & .00033 & \\
\hline $531 \mathrm{nHA}$ & 5305 & .8123 & .8121 & 20.9966 & 234.95 & .10350 & \\
\hline $53 \mid 424$ & 2356 & .0150 & .0146 & .1533 & 2.91 & .00128 & \\
\hline 531TUPF & 9996 & 1.3624 & 1,3823 & $7.147 !$ & 7.15 & $\begin{array}{l}.00315 \\
.11108\end{array}$ & .26 \\
\hline $532 y$ & $\lfloor 5\rfloor$ & .0088 & .0066 & .2129 & 3.09 & .00136 & \\
\hline $532 \times 4$ & 5325 & .8169 & (816) & 0.1949 & 236.29 & .10409 & \\
\hline $532+n$ & 2015 & .0128 & .0125 & .1247 & 2.37 & .00104 & \\
\hline 532nJPP & 14097 & 1.9426 & 1.9424 & 9.6051 & $9.3 !$ & $\begin{array}{l}.60423 \\
.11359\end{array}$ & .26 \\
\hline
\end{tabular}

182 때

\begin{tabular}{|c|c|c|c|c|c|c|c|c|}
\hline 5191 & 493 & .0066 & .0065 & .3509 & 5.05 & .00202 & & $\infty$ \\
\hline $519+1$ & 232 & .0016 & .0014 & .0210 & .31 & .00014 & & 25 \\
\hline $519 \%$ & 8207 & .9865 & .9863 & 50.0430 & 599.01 & .26124 & & 530 \\
\hline $519+x+4$ & 408 & .0261 & .0250 & .2676 & 5.08 & .01224 & & 25 \\
\hline $5204+$ & 440 & .0059 & 0057 & .2888 & 4.30 & $\begin{array}{l}.25348 \\
.00189\end{array}$ & .62 & 55 \\
\hline $560 \mathrm{H}$ & 8915 & .9038 & .9037 & 47.3892 & 585.26 & .25782 & & 562 \\
\hline \multirow[t]{2}{*}{ 520 $* 54$} & 469 & .0300 & .0298 & .2967 & 5.68 & .00250 & & 25 \\
\hline & & & & & & .26032 & .62 & \\
\hline 539: & 825 & .0089 & .0087 & .4578 & 6.64 & .00292 & & 147 \\
\hline 5334 & 65 & .0012 & .0010 & .0218 & .32 & .00014 & & $5 !$ \\
\hline 5331:4 & 8742 & .7264 & .1265 & 38.0866 & 439.38 & .18859 & & 567 \\
\hline $533+44$ & 2248 & .0340 & .0339 & . 3485 & 6.62 & .00292 & & \\
\hline \multirow[t]{2}{*}{$533 \pi U R ?$} & & $\mathbb{R R} R$ & ERROR & $\mathbb{R} R \mathbb{R}$ & ERROR & EPROR & & \\
\hline & & & & & & .19251 & .4 & \\
\hline $534 t$ & 300 & .0058 & .0056 & .2849 & 4.13 & .00162 & & 60 \\
\hline $534 \mathbf{H A}$ & 7245 & .6021 & .6019 & 30.5300 & 356.76 & .16157 & & 497 \\
\hline $53474++$ & 1093 & .0165 & .0169 & .1635 & 3.11 & .00137 & & \\
\hline \multirow[t]{2}{*}{$534 \mathrm{TUP}$} & & ERROR & DROR & ERROR & ZRPRO & ERPR? & & \\
\hline & & & & & & .16294 & .39 & \\
\hline
\end{tabular}




\section{TABLE A-15}

SEM/EDS Analyses Performed on ATM-1C, ATM-8 Glass

JEM/EOS analyses were performed on the samples shown in Table A-15. These analyses included profiling pollshed cross-seations and exanining reacted. The compilation of photomisrographs, spectra, and line profile analyses is maintalned by J. Bates 
Taivie A-15. SEM/EDS Analyses Performed on ATM-1C, aTM-8 Glass

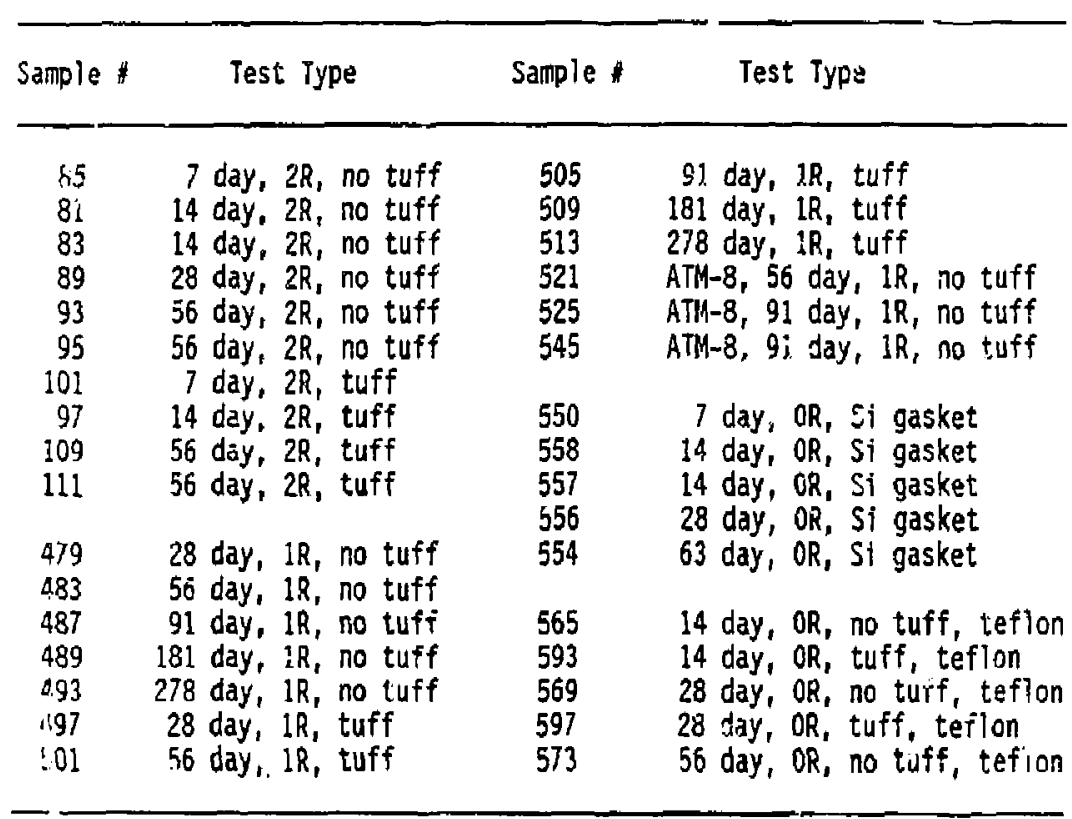

$a_{A} 11$ samples were ATM-ic unless noted. 


\section{TABLE A-16}

ATM-1c Glasses Profiled Using SIMS

SIMS profiles were collected for the samples listed in Table A-16. The profiles were collected using a $4 \mathrm{keV} \mathrm{Ar}^{+}$beam that was rastered over the surface. The sputtered fons were collected only from the central portion of the rastered area. The sample was charge neutralized using an electron flood gun. Spectra were collected for two mass ranges, 0-75 and 65-285 AMU. Two minutes were required to collect a spectrum. Spectra were collected with a measured delay between each spectrum depending or the anticipated thickness of the reacted layer.

The raw data were collected as real-time $X-Y$ plots and also stored in a computer. The computer collected data were processed to obtain background corrected intensities for each peak of interest. These intensities were then ratioed to a peak whose absolute intensity remained relatively constant across the reacted layer. In reality, the concentration of no element was constant in all samples and $S 1(m / e=28)$ was usually chosen as the peak for ratioing in the 0-75 AMU range and $\mathrm{Si}_{2} \mathrm{O}(\mathrm{m} / \mathrm{e}=72)$ was used in the 65-285 AMU range. The ratioed intensities were then plotted as a function of spectrum number, which could be correlated to depth by the use of a calculated sputtering rate. It was generally noted that the intensity of $\mathrm{Si}$ or $\mathrm{Si}_{2} \mathrm{O}$ was greatest in the first several spectra $(<0.2 \mu \mathrm{m})$ and was constant thereafter.

Examples of selected spectra and ratioed profiles are shown in Figs. A-1, A-2, and A-3. Highlighted in the spectra are those elements whose intensities show a substantial change between the reacted layer and the butk glass. 
Table A-16. ATM-1C Glasses Proftled Using SIMS

\begin{tabular}{cccc}
\hline Sample * & Test Type & Data Storage & Date collected \\
\hline 549 & 7 day, OR, Si gasket & 549 & $12 / . / 86$ \\
557 & 14 day, OR, S1 gasket & $549 \mathrm{~A}$ & $12 / 22 / 86$ \\
563 & 28 day, OR, Si gasket & $557 \mathrm{~A}$ & $12 / 30 / 86$ \\
& & $563 \mathrm{~A}$ & $12 / 31 / 86$ \\
& & $563 \mathrm{~B}$ & $1 / 08 / 87$ \\
562 & 63 day, OR, Si gasket & 562 & $1 / 15 / 87$ \\
& & $562 \mathrm{~A}$ & $6 / 11 / 87$ \\
88 & 7 day, 2R & 88 & $6 / 11 / 87$ \\
& & $88 \mathrm{~A}$ & $5 / 21 / 87$ \\
92 & 28 day, 2R & 92 & $5 / 21 / 87$ \\
96 & 56 day, 2R & $92 \mathrm{~A}$ & $5 / 21 / 87$ \\
& & 96 & $6 / 21 / 87$ \\
& & $96 \mathrm{~A}$ & $6 / 12 / 87$ \\
\hline
\end{tabular}


118

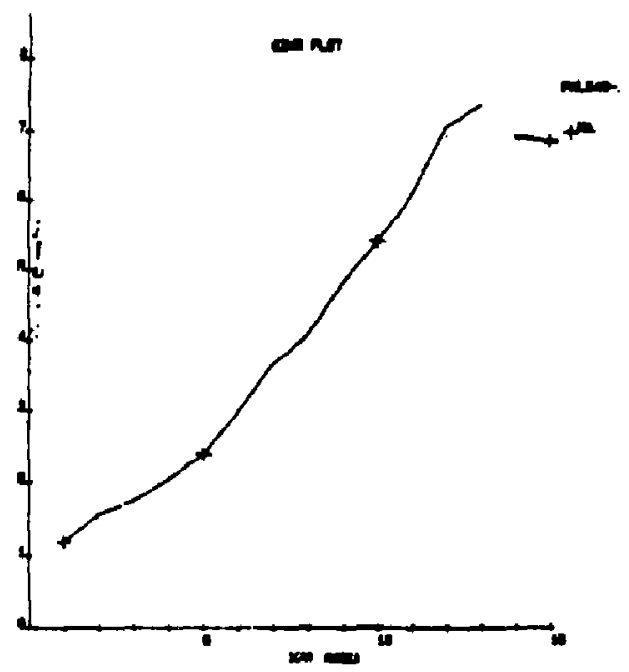

(a)

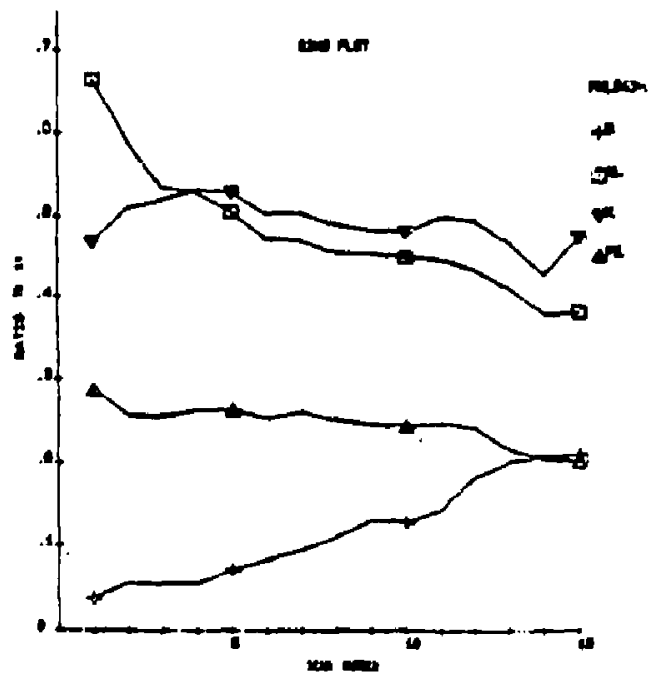

(b)

Fig. A-la-f. SIMS Profiles for (a) Na and (b) B, Al, K, and Fe for ATM-1C. Glass Reacted for 7 Days at 0 Rh; SIMS Profiles for $(c) \mathrm{N}$ ", $\mathrm{Ca}$; (d) $\mathrm{B}, \mathrm{Mg}, \mathrm{Cs}$; (e) Cs, Sr. Ba, Re; and (f) Ink, Mo, U for ATM-1C Glass Reacted for 14 Days at $0 \mathrm{R} / \mathrm{h}$. 


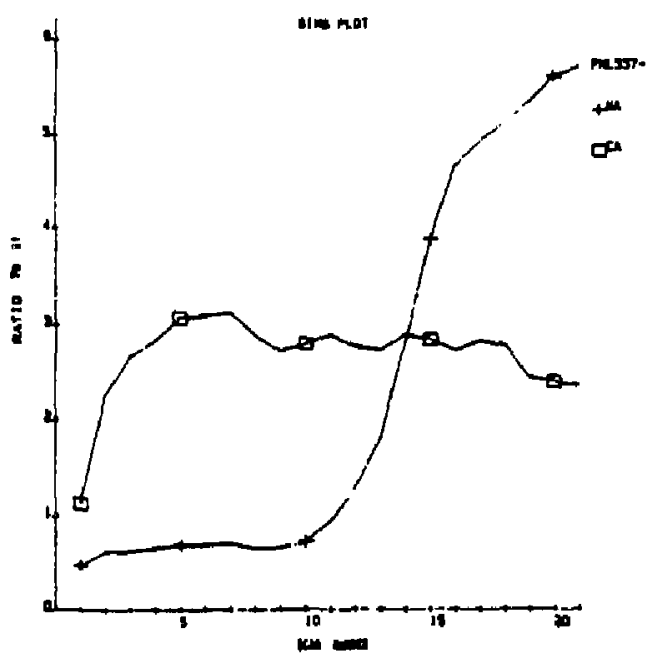

(c)

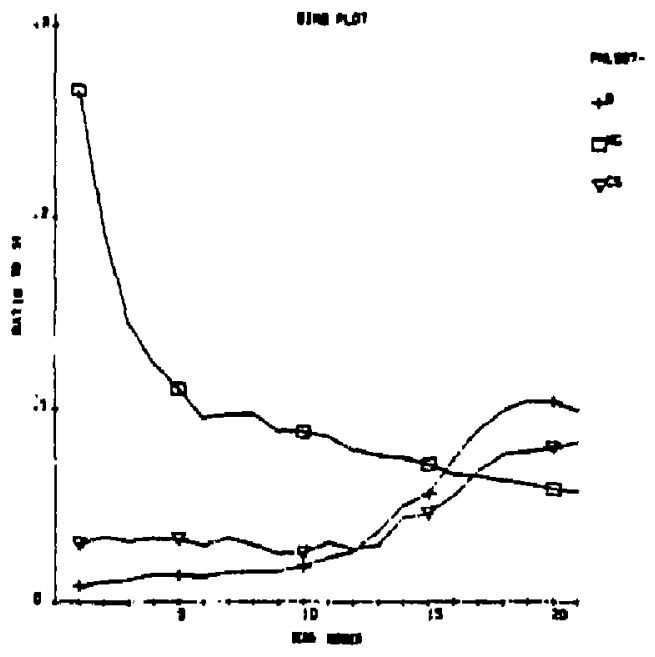

(d)

Fig. A-1 (Cont'd)

$c$ and $d$ 


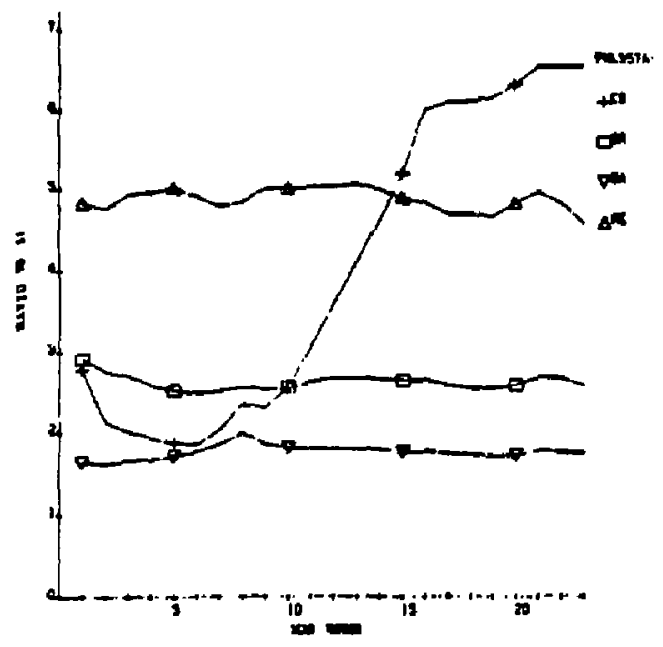

(e)

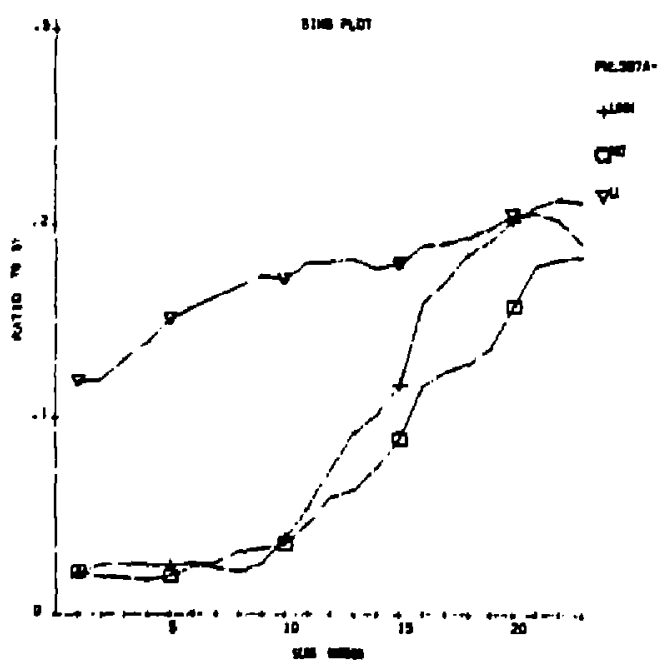

(f)

Fig. A-1 (Cant'd)

$e$ and $f$ 


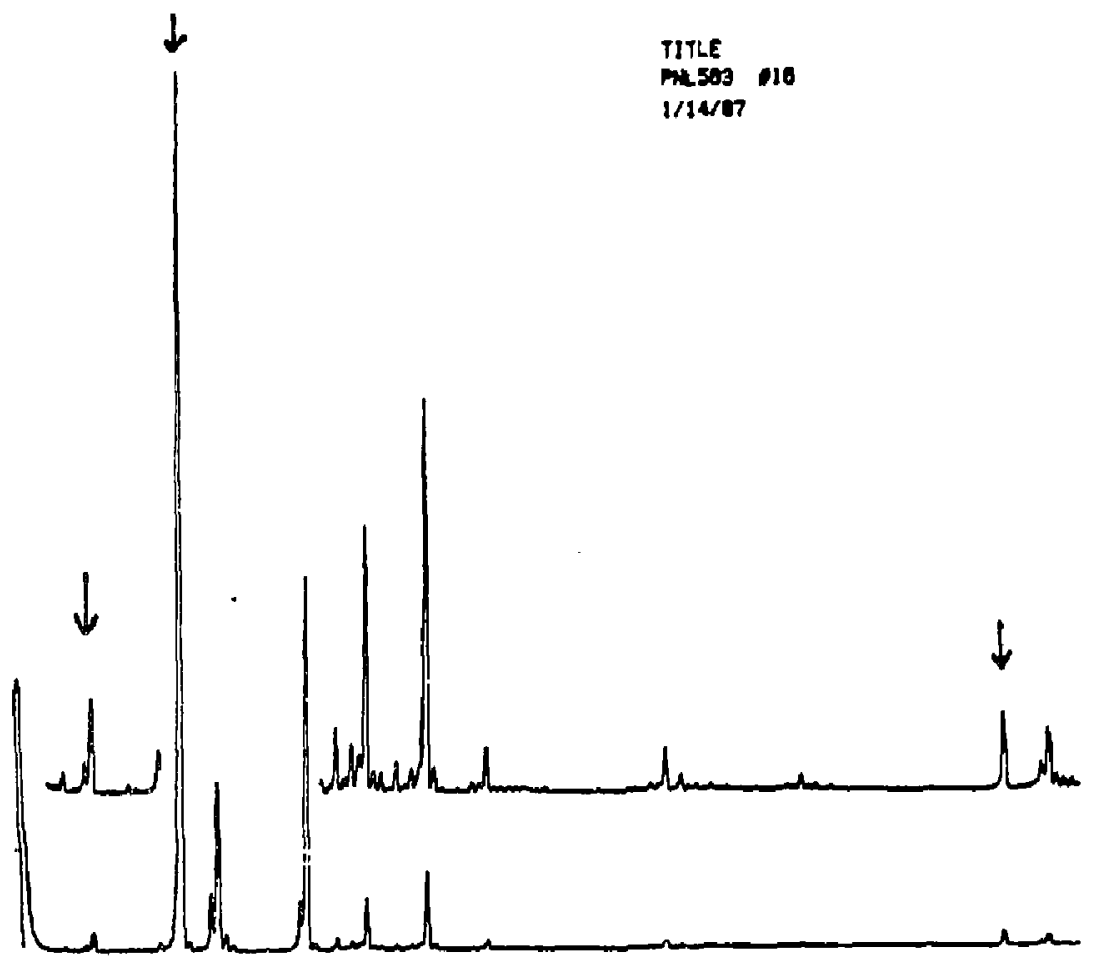

(a)

Fig. A-2. SIMS Spectra of (a) Reacted Layer and (b) Bulk Glass of ATM-1c Glass. Those elements that change noticeably are highlighted. The mass range is $0-150$ amu. 


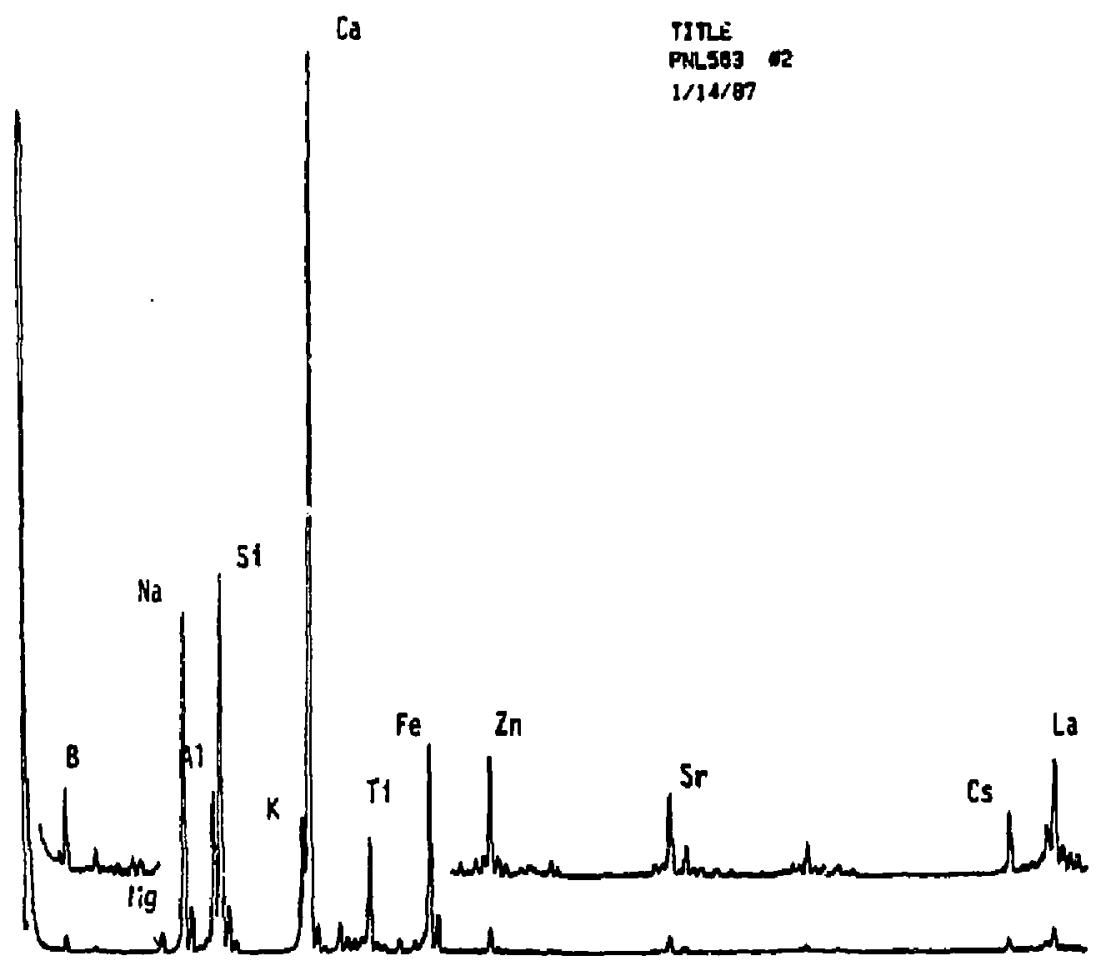

(b)

Fig. A-2 (Cont'd) 


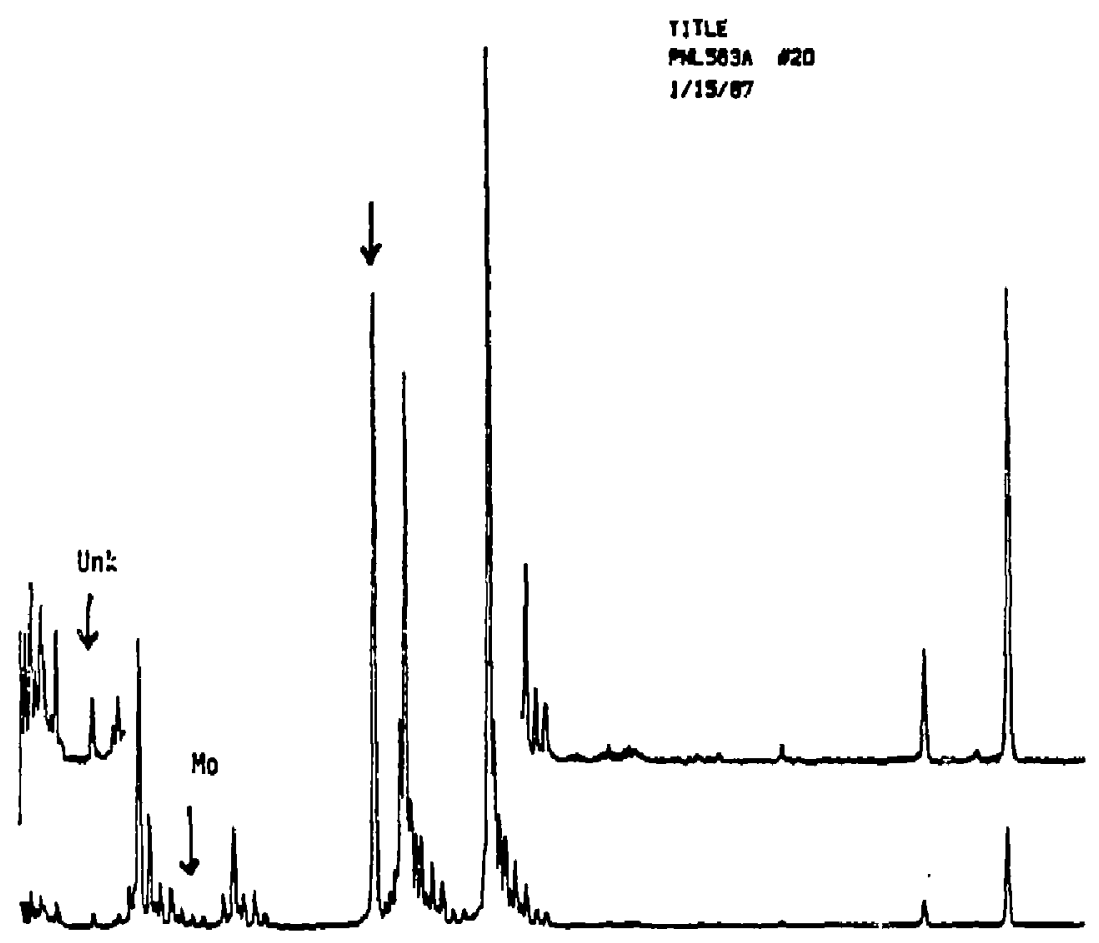

(a)

Fig. A-3. SIMS Spectra of (a) Reacted Layer and (b) Bulk Glass of ATM-ic Glass. Those elements that change noticeably are highlighted. The mass range is 65-275 amu. 


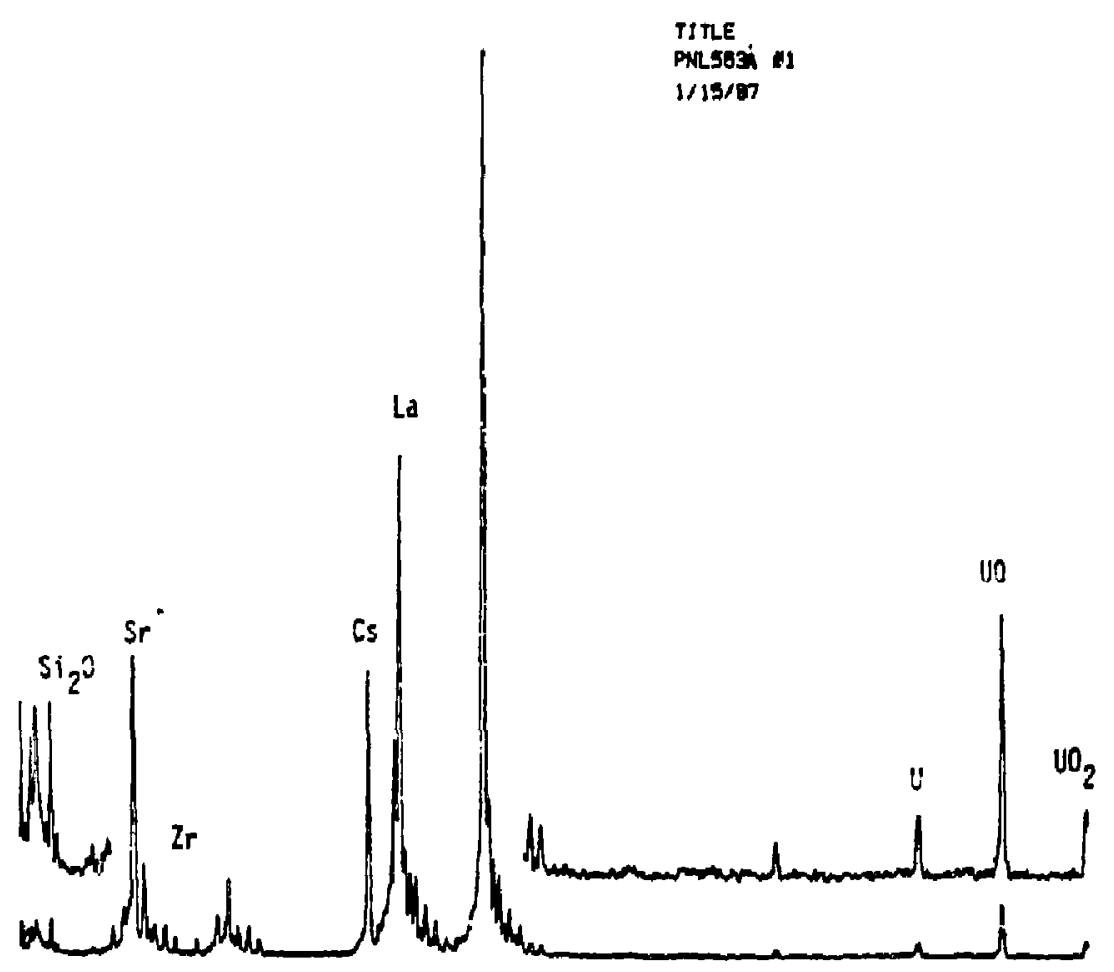

(b)

Fig. A-3 (Cont'd) 


\section{REFERENCES}

\section{BATES-1}

J. K. Bates, H. L. Ebert, D. F. Fischer, and T. J. Gerding, "The Reaction of Reference Commercial Nuclear Waste Glasses during Gama Irradiation in a Saturated Tuff Environment," submitted to the Journal of Materials Research (1987).

\section{BATES-2}

J. K. Bates, D. F. Fischer, and T. J. Gerding, "The Reaction of Glass during Ganma Irradiation in a Saturated Tuff Environment, Part 1: SRL 165 Glass," Argonne National Laboratory Report ANL-85-62 (1986). 